\title{
A Unified Approach to the Chemoselective $\alpha-$ Functionalization of Amides with Heteroatom Nucleophiles
}

\author{
Carlos R. Gonçalves, Miran Lemmerer, Christopher J. Teskey, Pauline Adler, Daniel Kaiser, \\ Boris Maryasin, Leticia González and Nuno Maulide*
}

Supporting Information 


\section{Contents}

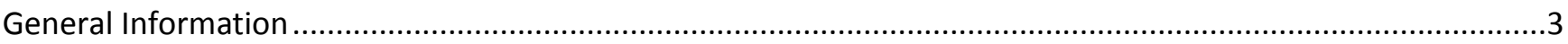

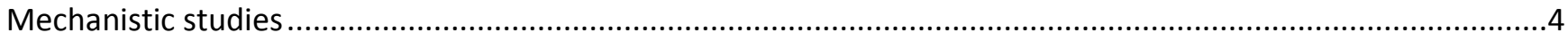

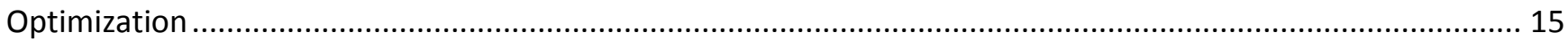

General procedures for the synthesis of carboxamides and sulfonamides ............................................... 16

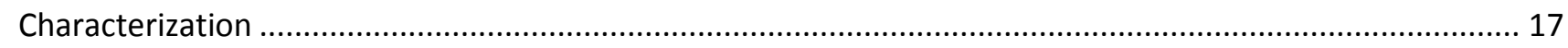

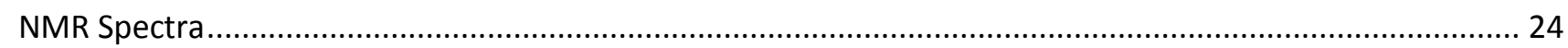

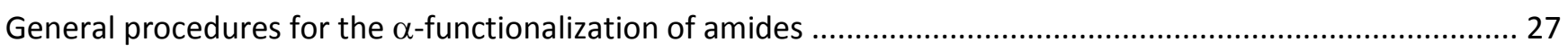

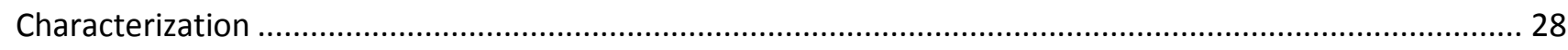

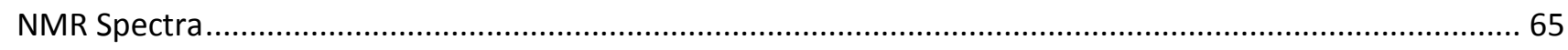

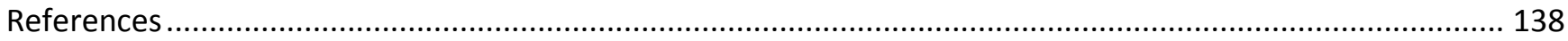

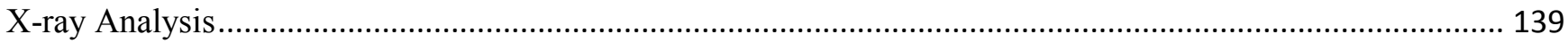

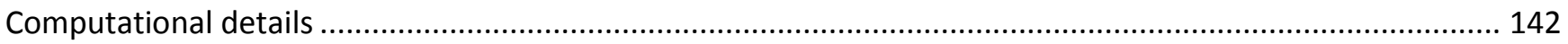

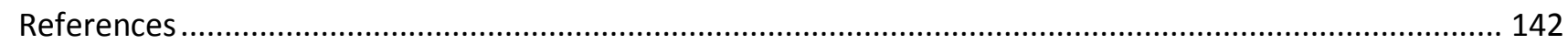

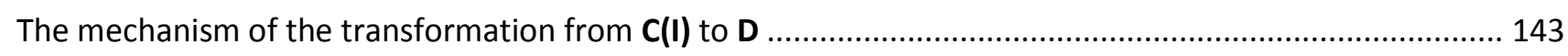

Coordinates of the most stable $\left(\Delta G_{298, D C M}\right)$ conformations as computed at the DLPNO-CCSD(T)/def2-TZVP//B3LYP-D3/def2-SVP level of theory ......................................................... 144 


\section{General Information}

Unless otherwise stated, all glassware was flame-dried before use and all reactions were performed under an atmosphere of argon. Triflic anhydride was distilled over $\mathrm{P}_{4} \mathrm{O}_{10}$ prior to use. All other reagents were used as received from commercial suppliers unless otherwise stated. Reaction progress was monitored by thin layer chromatography (TLC) performed on aluminium plates coated with silica gel F254 with $0.2 \mathrm{~mm}$ thickness. Chromatograms were visualized by fluorescence quenching with UV light at $254 \mathrm{~nm}$ or by staining using potassium permanganate. Flash column chromatography was performed using silica gel 60 (230-400 mesh, Merck and co.). Neat infra-red spectra were recorded using a Perkin-Elmer Spectrum 100 FT-IR spectrometer. Wavenumbers (vmax) are reported in $\mathrm{cm}^{-1}$. Mass spectra were obtained using a Finnigan MAT 8200 or (70 eV) or an Agilent 5973 (70 eV) spectrometer, using electrospray ionization (ESI). All ${ }^{1} \mathrm{H}$ NMR and ${ }^{13} \mathrm{C}$ NMR spectra were recorded using a Bruker AV-400, AV-600 and AV-700 spectrometer at 300K. Chemical shifts were given in parts per million (ppm, $\delta$ ), referenced to the solvent peak of $\mathrm{CDCl}_{3}$, defined at $\delta=7.26 \mathrm{ppm}\left({ }^{1} \mathrm{H} \mathrm{NMR}\right)$ and $\delta=$ $77.16\left({ }^{13} \mathrm{C}\right.$ NMR). Coupling constants are quoted in $\mathrm{Hz}(\mathrm{J}) .{ }^{1} \mathrm{H}$ NMR splitting patterns were designated as singlet $(s)$, doublet $(d)$, triplet $(t)$, quartet $(q)$, pentet $(p)$. Splitting patterns that could not be interpreted or easily visualized were designated as multiplet $(\mathrm{m})$ or broad $(\mathrm{br})$. Selected ${ }^{13} \mathrm{C}$ NMR spectra were recorded using the attached proton test (APT) to facilitate the confirmation and assignment of the structure.

\section{General Procedure for Nucleophilic $\alpha$-Functionalisation of Amides}

To a mixture of amide $(0.20 \mathrm{mmol})$, 2-iodopyridine $(0.44 \mathrm{mmol})$ in DCM $(2.0 \mathrm{~mL})$ was added triflic anhydride (37 $\mu \mathrm{L}, 0.22 \mathrm{mmol}$ ) dropwise under $\mathrm{Ar}$ at $0{ }^{\circ} \mathrm{C}$. After stirring for $15 \mathrm{~min}$ at $0{ }^{\circ} \mathrm{C}, 2,6$-lutidine $N$-oxide $(22.4 \mu \mathrm{L}, 0.20$ $\mathrm{mmol}$, ) was added and the reaction stirred for a further $5 \mathrm{~min}$ at $0{ }^{\circ} \mathrm{C}$. Then the nucleophile (vide infra for details) was added in one portion ( 3 equiv.). The reaction mixture was stirred at room temperature for $3 \mathrm{~h}$ before being quenched with $\mathrm{NH}_{4} \mathrm{Cl}$ solution. The layers were separated and the aqueous extracted with $\mathrm{DCM}$. The combined organic layers were washed with brine before being dried over anhydrous $\mathrm{MgSO}_{4}$. The solvent was removed under reduced pressure. The crude product was purified by column chromatography. 


\section{Mechanistic studies}

\section{2o: 2-hydroxy-4-phenyl-1-(pyrrolidin-1-yl)butan-1-one}<smiles>O=C(CCc1ccccc1)C(O)C1CCCC1</smiles>

A flamed dried Schelenk tube containing 4-Phenyl-1-(pyrrolidin-1-yl)butan-1-one (0.434 g, $2.0 \mathrm{mmol}$ ) in $25 \mathrm{~mL}$ of DCM was cooled down to $-78^{\circ} \mathrm{C}$ for 15 minutes before the addition of 1.2 equiv. of LiHMDS (2.1 mL, $1 \mathrm{M}$ in DCM). The mixture was stirred for 1 hour before oxygen gas saturated the solution. After 3 hours the reaction was then allowed to slowly warm up to $\mathrm{rt}$. At this point 3 equiv. of triethylphosphite were added. After 1 hour the mixture was quenched with saturated $\mathrm{NH}_{4} \mathrm{Cl}$ and washed $3 x$ times with $\mathrm{DCM}$. The combined organic phases were dried using $\mathrm{MgSO}_{4}$ and evaporated. Purification by column chromatography on silica gel (EtOAc: heptane $=2: 1$ ) yielded the product as a yellow oil $(0.251 \mathrm{~g}, 54 \%)$.

${ }^{1} \mathrm{H}$ NMR (400 MHz, $\left.\mathrm{CDCl}_{3}\right): \delta 7.30-7.27(\mathrm{~m}, 3 \mathrm{H}), 7.24-7.16(\mathrm{~m}, 2 \mathrm{H}), 4.17-4.14(\mathrm{~m}, 1 \mathrm{H}), 3.71(\mathrm{~d}, J=7.2 \mathrm{~Hz}, 1 \mathrm{H})$, $3.55-3.52(\mathrm{~m}, 1 \mathrm{H}), 3.43-3.39(\mathrm{~m}, 1 \mathrm{H}), 3.31-3.26(\mathrm{~m}, 1 \mathrm{H}), 3.19-3.14(\mathrm{~m}, 1 \mathrm{H}), 2.89-2.77(\mathrm{~m}, 2 \mathrm{H}), 1.95-1.79$ $(\mathrm{m}, 6 \mathrm{H}) ;{ }^{13} \mathrm{C}$ NMR (151 MHz, $\left.\mathrm{CDCl}_{3}\right): \delta 172.9,141.6,128.7$ (2C) , 128.6 (2C) , $126.2,68.6,46.4,45.9,36.2,31.4$ , 26.2 , 24.0; HRMS (ESI): calculated for $[\mathrm{M}+\mathrm{H}]^{+}\left(\mathrm{C}_{14} \mathrm{H}_{19} \mathrm{NO}_{2}\right) \mathrm{m} / \mathrm{z}: 256.1313$ found $[\mathrm{M}+\mathrm{H}]^{+}: 256.1316$; IR (neat, $\mathrm{cm}^{-1}$ ): 3400, 3059, 2936, 1645. 

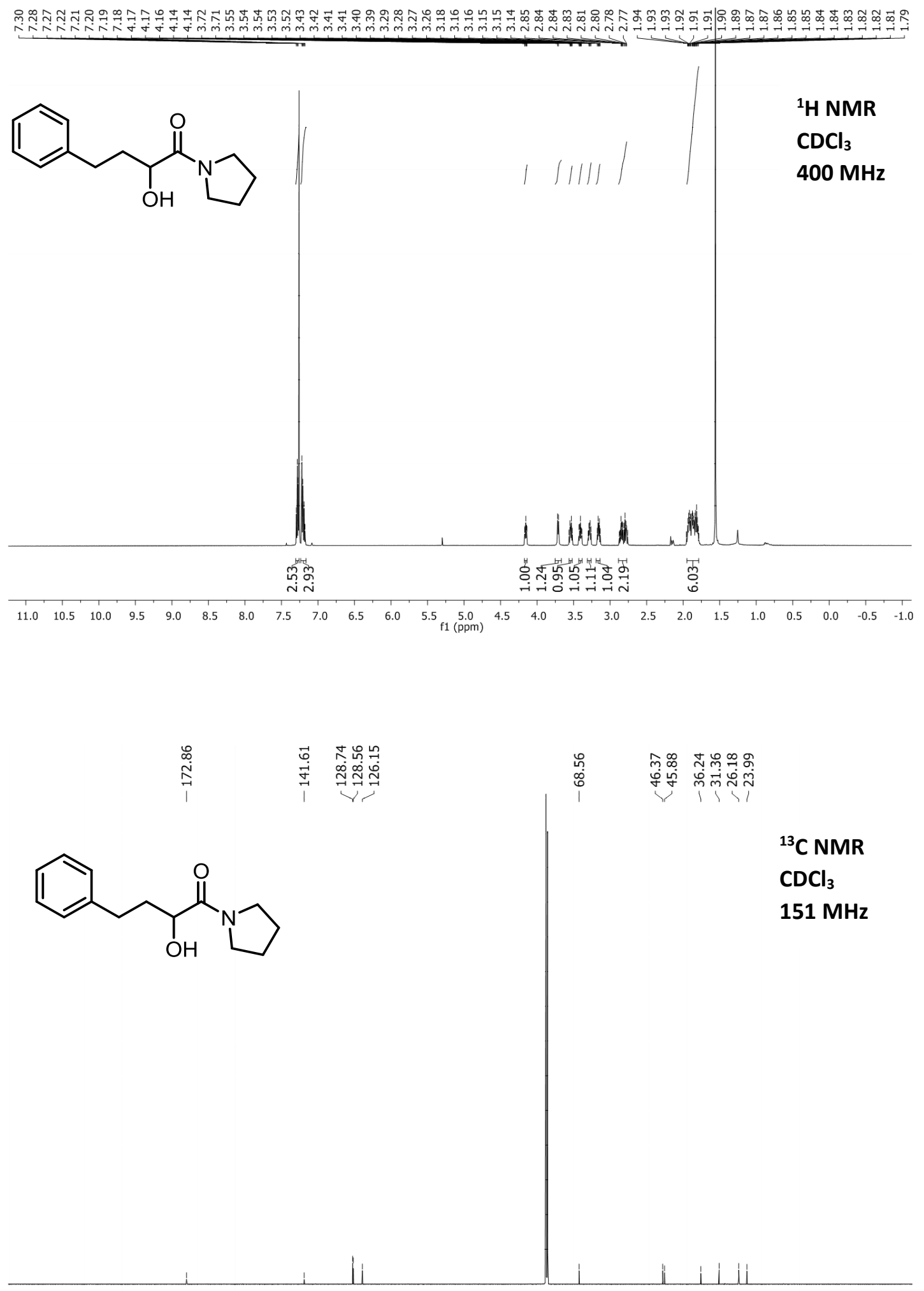

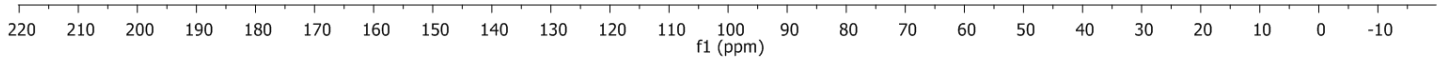


Independent formation and isolation of the $\alpha$-triflate amide.

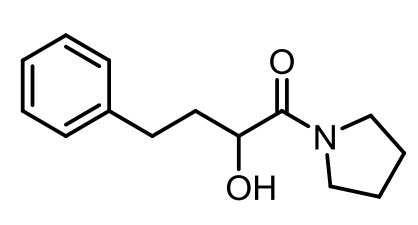

1.1 equiv. $\mathrm{Tf}_{2} \mathrm{O}$

1.0 equiv. 2,6 -lutidine

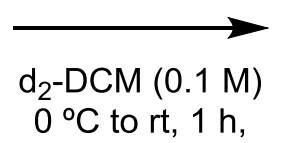

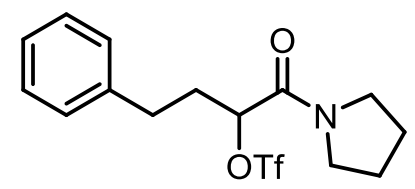

To a solution of the amide $2 \mathrm{~m}\left(0.2 \mathrm{mmol}, 1\right.$ equiv.) and distilled lutidine (1 equiv.) in $\mathrm{d}_{2}-\mathrm{DCM}(0.1 \mathrm{M}, 2 \mathrm{~mL})$ at $0{ }^{\circ} \mathrm{C}$, triflic anhydride ( $35 \mu \mathrm{L}, 1.1$ equiv.) was added dropwise and the resulting reaction mixture was allowed to warm to room temperature while stirring for 1 hour. After this time, the reaction mixture was quickly purified by column chromatography (Ethyl acetate/heptane $1: 1-3: 1$ ) at $-30^{\circ} \mathrm{C}$ (cryostat - isopropanol) to afford the alpha triflate amide in $70 \%$ yield $(50.0 \mathrm{mg})$ as a yellow oil.<smiles>O=C(C(Br)CCc1ccccc1)N1CCCC1</smiles>

${ }^{1} \mathrm{H}$ NMR (400 MHz, $\left.\mathrm{CDCl}_{3}\right): \delta 7.36-7.28(\mathrm{~m}, J=8.5,1.5 \mathrm{~Hz}, 2 \mathrm{H}), 7.25-7.17(\mathrm{~m}, 3 \mathrm{H}), 5.21(\mathrm{dd}, J=8.7,4.4 \mathrm{~Hz}, 1 \mathrm{H})$, $3.62-3.48(\mathrm{~m}, 1 \mathrm{H}), 3.48-3.36(\mathrm{~m}, 2 \mathrm{H}), 3.14-3.04(\mathrm{~m}, \mathrm{~J}=9.9,6.9 \mathrm{~Hz}, 1 \mathrm{H}), 2.91-2.80(\mathrm{~m}, J=14.3,8.8,5.6 \mathrm{~Hz}$, $1 \mathrm{H}), 2.78-2.67(\mathrm{~m}, 1 \mathrm{H}), 2.46-2.33(\mathrm{~m}, \mathrm{~J}=14.2,8.6,5.6 \mathrm{~Hz}, 1 \mathrm{H}), 2.24-2.14(\mathrm{~m}, 1 \mathrm{H}), 1.94-1.79(\mathrm{~m}, 4 \mathrm{H}) ;{ }^{13} \mathrm{C}$ NMR (151 MHz, CDCl $): \delta 164.5,139.3,128.9,128.7,128.6,128.5,126.9,118.6$ (q, J= $320 \mathrm{~Hz}$ ), 83.1 , 46.6 , 46.2 , $33.4,30.8,26.2,23.9 ;{ }^{19} \mathrm{~F} \mathrm{NMR}\left(565 \mathrm{MHz} \mathrm{CDCl}_{3}\right.$ ): $\delta$-75.4, -78.9 (OTf); HRMS (ESI): calculated for [M+H] ${ }^{+}$ $\left(\mathrm{C}_{15} \mathrm{H}_{19} \mathrm{~F}_{3} \mathrm{NO}_{4} \mathrm{~S}\right) \mathrm{m} / \mathrm{z}: 366.0981$ found $[\mathrm{M}+\mathrm{H}]^{+}: 366.0969$. 


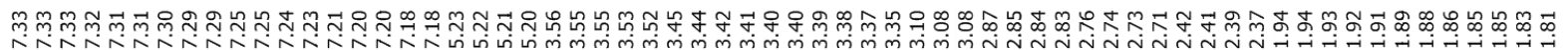<smiles>O=C(CCc1ccccc1)C(Br)C1CCCC1</smiles>

$$
\begin{aligned}
& { }^{1} \mathrm{H} \mathrm{NMR} \mathrm{CDCl}_{3} \\
& 400 \mathrm{MHz}^{2}
\end{aligned}
$$
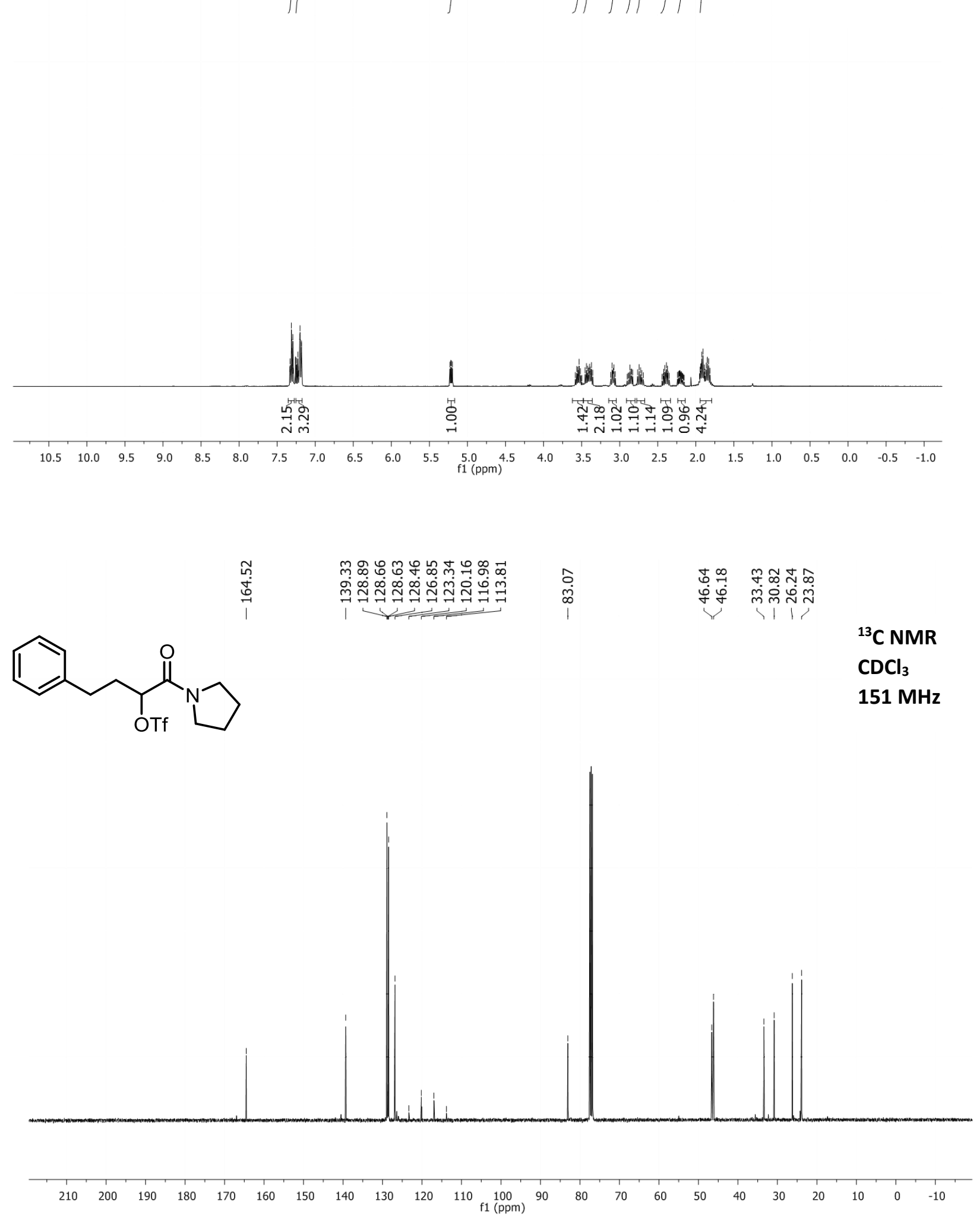

S7 


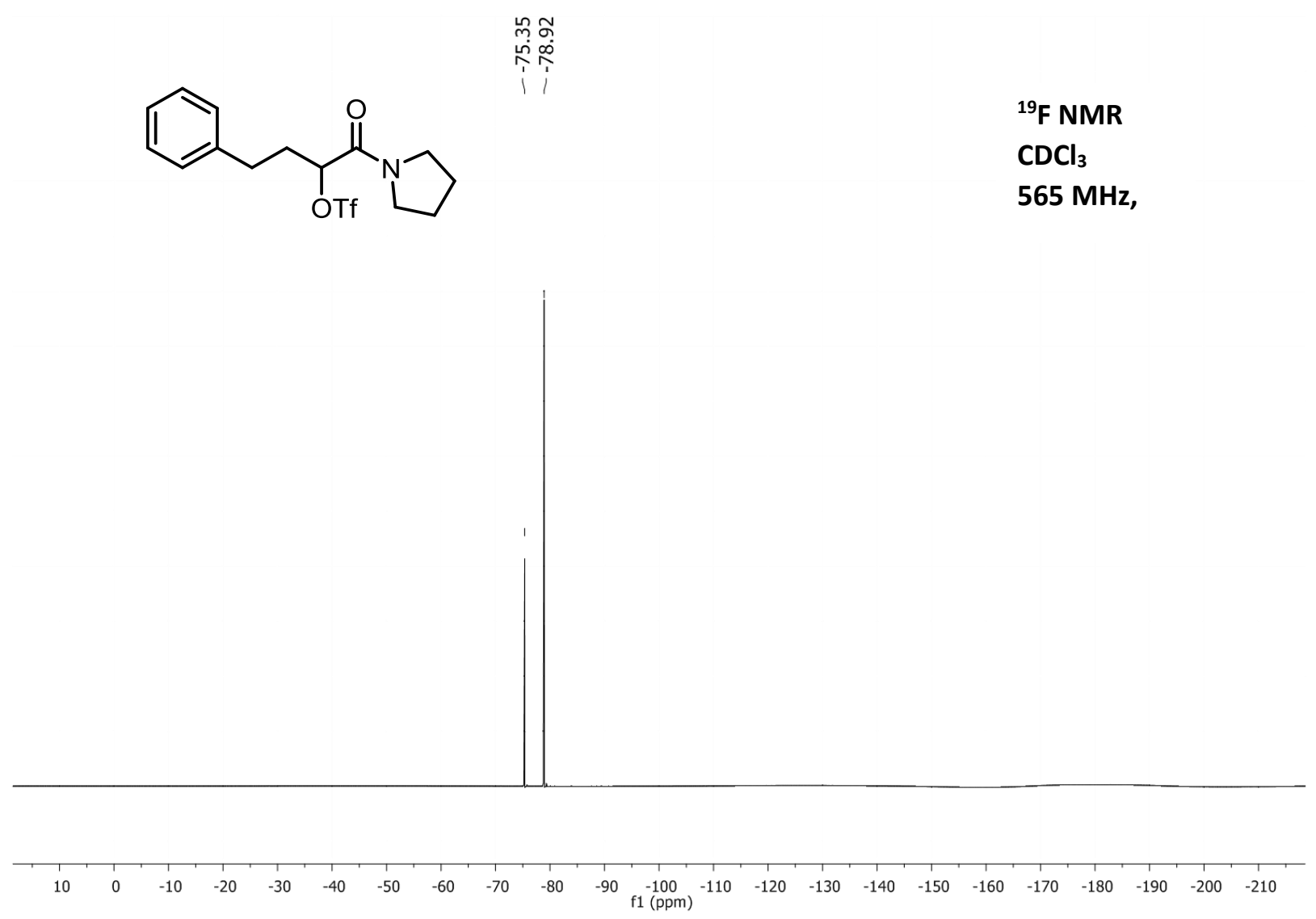

\section{$N, N$-Dibenzyl-2-hydroxy-4-phenylbutanamide}<smiles>O=C(C(O)CCc1ccccc1)N(Cc1ccccc1)Cc1ccccc1</smiles>

A flamed dried Schelenk tube containing $N, N$-dibenzyl-4-phenylbutanamide $(0.69 \mathrm{~g}, 2.0 \mathrm{mmol})$ in $25 \mathrm{~mL}$ of DCM was cooled down to $-78^{\circ} \mathrm{C}$ for 15 minutes before the addition of 1.2 equiv. of LiHMDS (2.1 mL, $1 \mathrm{M}$ in DCM). The mixture was stirred for 1 hour before oxygen gas saturated the solution. After 3 hours the reaction was then allowed to slowly warm up to rt. At this point 3 equiv. of triethylphosphite were added. After 1 hour the mixture was quenched with saturated $\mathrm{NH}_{4} \mathrm{Cl}$ and washed $3 x$ times with $\mathrm{DCM}$. The combined organic phases were dried using $\mathrm{MgSO}_{4}$ and evaporated. Purification by column chromatography on silica gel (EtOAc: heptane $=2: 1$ ) yielded the product as a yellow oil $(0.373 \mathrm{~g}, 52 \%)$. 
${ }^{1} \mathrm{H}$ NMR $\left(600 \mathrm{MHz}, \mathrm{CDCl}_{3}\right): \delta 7.38-7.26(\mathrm{~m}, 6 \mathrm{H}), 7.25-7.23(\mathrm{~m}, 2 \mathrm{H}), 7.17-7.15(\mathrm{~m}, 3 \mathrm{H}), 7.12-7.11(\mathrm{~m}, 2 \mathrm{H})$, $7.04-7.03(\mathrm{~m}, 2 \mathrm{H}), 4.98(\mathrm{~d}, J=14.8 \mathrm{~Hz}, 1 \mathrm{H}), 4.39(\mathrm{dd}, J=8.6,2.6 \mathrm{~Hz}, 1 \mathrm{H}), 4.27(\mathrm{~d}, J=16.6 \mathrm{~Hz}, 1 \mathrm{H}), 4.21(\mathrm{~d}, J=$ $14.8 \mathrm{~Hz}, 1 \mathrm{H}), 4.10(\mathrm{~d}, J=16.6 \mathrm{~Hz}, 1 \mathrm{H}), 3.92-3.53(\mathrm{brs}, 1 \mathrm{H}), 2.87-2.76(\mathrm{~m}, 2 \mathrm{H}), 1.97-1.92(\mathrm{~m}, 1 \mathrm{H}), 1.92-1.82$ (m, 1H); ${ }^{13} \mathrm{C}$ NMR (151 MHz, CDCl 3 ): $\delta 175.4,141.3,136.6,135.4,129.2$ (C2) , 128.9 (C2) , 128.7 (C2) , 128.6 (C2), 128.4 (C2) , 128.1 , $127.9,126.8$ (2C) , 126.2 , $67.5,48.9$, 48.4 , 37.5 , 31.4; HRMS (ESI): calculated for $[\mathrm{M}+\mathrm{Na}]^{+}\left(\mathrm{C}_{24} \mathrm{H}_{25} \mathrm{NONa}\right) \mathrm{m} / \mathrm{z}: 366.1834$ found $[\mathrm{M}+\mathrm{Na}]^{+}: 366.1823$; IR (neat, $\left.\mathrm{cm}^{-1}\right): 3062,3032,2928,1644,1494$, 1452.

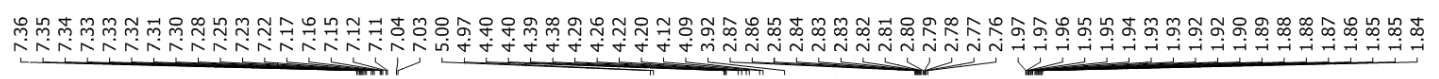

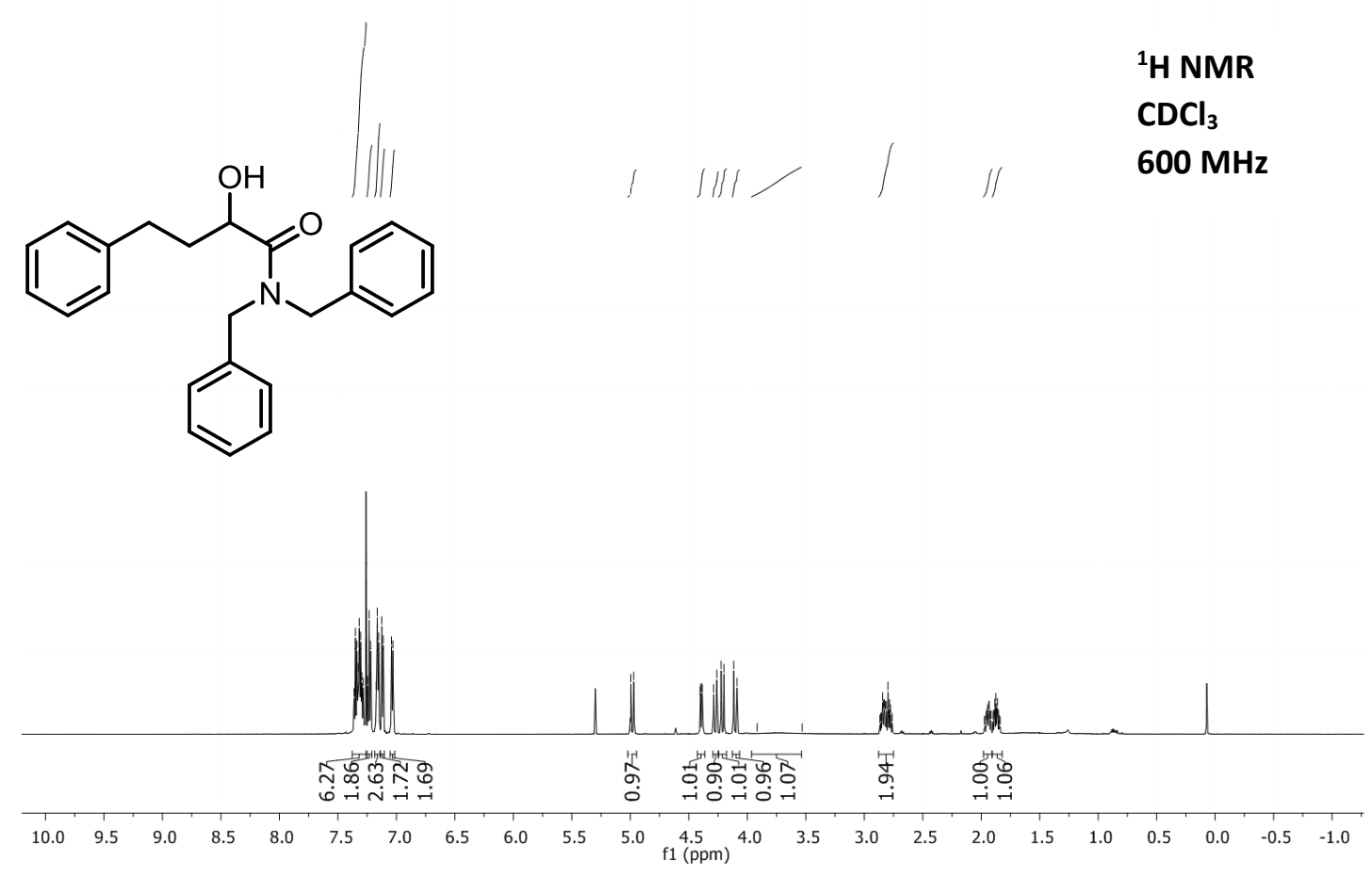




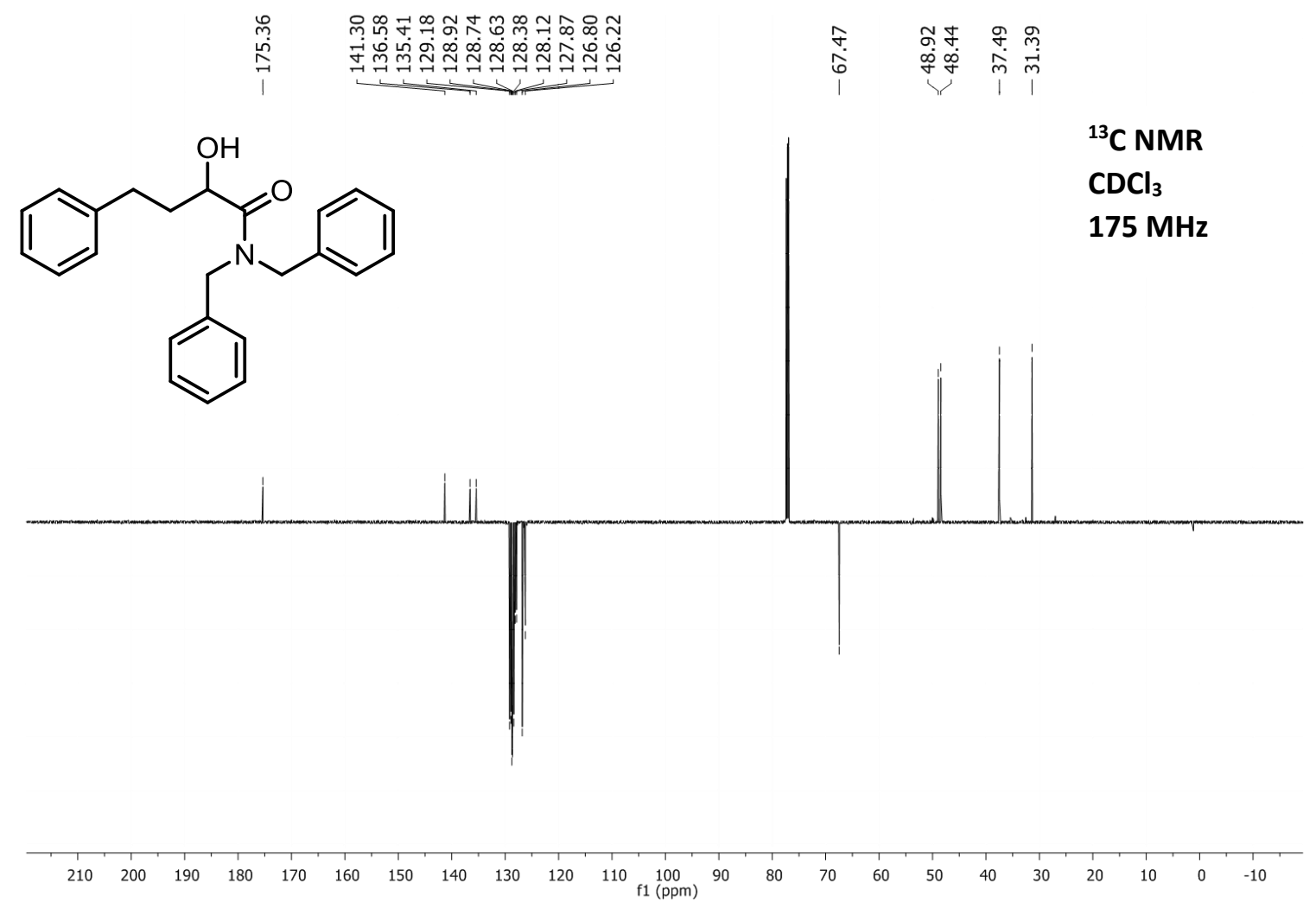




\section{Isolation of the alpha triflate amide from an Umpolung reaction.}
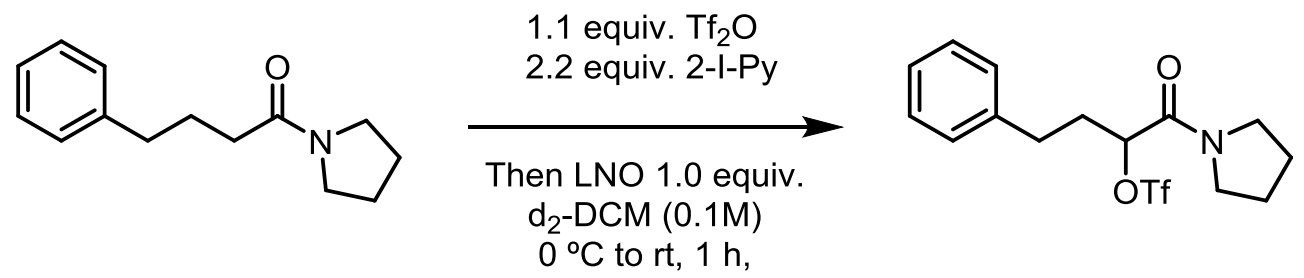

To a mixture of amide 4-Phenyl-1-(pyrrolidin-1-yl)butan-1-one $(0.2 \mathrm{mmol}), 2$-iodopyridine $(0.44 \mathrm{mmol})$ in DCM $(2 \mathrm{~mL})$ was added triflic anhydride $(37 \mu \mathrm{L}, 0.22 \mathrm{mmol})$ dropwise under $\operatorname{Ar}$ at $0{ }^{\circ} \mathrm{C}$. After stirring for $15 \mathrm{~min}$ at $0{ }^{\circ} \mathrm{C}$, lutidine $N$-oxide $(22.4 \mu \mathrm{L}, 0.2 \mathrm{mmol})$ was added and the reaction stirred for a further $5 \mathrm{~min}$ at $0{ }^{\circ} \mathrm{C}$. After 1 hour the reaction was quenched with water and extracted $2 \mathrm{x}$ with DCM. The combined organic layers were dried over anhydrous $\mathrm{MgSO}_{4}$. The solvent was removed under reduced pressure. The crude mixture was quickly columned at $-30{ }^{\circ} \mathrm{C}$ (cryostat / isopropanol) to afford the desired compound in $95 \%$ yield (69.0 $\mathrm{mg}$ ) as a yellow oil.

\section{Disclosure}

One important fact to disclose was that the formation of the alpha triflate from the corresponding alpha hydroxyamide proved to proceed in consistently higher yields when 2,6-lutidine was used as a base. For instance, when 2-I-Pyridine was used the yield was significantly lower and the ${ }^{1} \mathrm{H}-\mathrm{NMR}$ analysis became less straightforward.

In order to access whether the intermediate ( $\alpha$-triflate) could be transformed to the intramolecular fridelcraft product we scoped a wide array of conditions. We soon realized that forcing conditions, such as the one encountered previously in the literature (enolnium ref paper) where ideal. After careful examination we became aware that 2-I-Pyridine as a base, combined with extreme temperatures $\left(110^{\circ} \mathrm{C}\right)$ in acetonitrile were crucial for the reaction to take place (vide infra). 


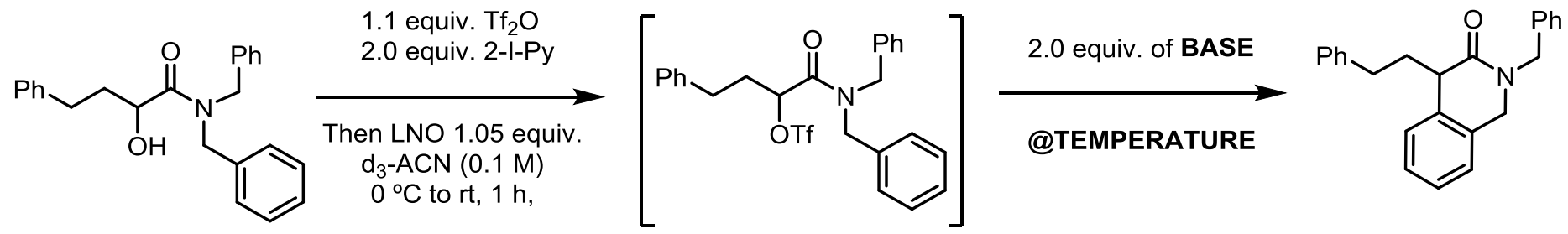

\begin{tabular}{|l|l|l|l|}
\hline Entry & Base & Temperature $\left({ }^{\circ} \mathrm{C}\right)$ & Yield (NMR) \\
\hline 1 & $2,6-$ Iutidine & 0 & - \\
\hline 2 & $2,6-$ Iutidine & 110 & - \\
\hline 3 & 2-I-Pyridine & 0 & - \\
\hline 4 & 2-I-Pyridine & 80 & - \\
\hline 5 & 2-I-Pyridine & 110 (3 hours) & 18 \\
\hline 6 & 2-I-Pyridine & 110 (14 hours) & 47 \\
\hline
\end{tabular}

\section{2-benzyl-4-phenethyl-1,4-dihydroisoquinolin-3(2H)-one}<smiles>O=C1C(CCc2ccccc2)c2ccccc2CN1Cc1ccccc1</smiles>

${ }^{1} \mathrm{H}$ NMR $\left(600 \mathrm{MHz}, \mathrm{CDCl}_{3}\right): \delta 7.37-7.26(\mathrm{~m}, 7 \mathrm{H}), 7.25-7.19(\mathrm{~m}, 2 \mathrm{H}), 7.17(\mathrm{dd}, J=14.0,7.0 \mathrm{~Hz}, 4 \mathrm{H}), 7.09(\mathrm{~d}, J=$ $7.7 \mathrm{~Hz}, 1 \mathrm{H}), 4.88(\mathrm{~d}, J=14.8 \mathrm{~Hz}, 1 \mathrm{H}), 4.64(\mathrm{~d}, J=14.8 \mathrm{~Hz}, 1 \mathrm{H}), 4.54(\mathrm{~d}, J=15.9 \mathrm{~Hz}, 1 \mathrm{H}), 4.21(\mathrm{~d}, J=15.9 \mathrm{~Hz}, 1 \mathrm{H})$, $3.70(\mathrm{t}, J=6.8 \mathrm{~Hz}, 1 \mathrm{H}), 2.70-2.64(\mathrm{~m}, 2 \mathrm{H}), 2.25-2.14(\mathrm{~m}, 1 \mathrm{H}), 2.14-2.01(\mathrm{~m}, 1 \mathrm{H}) ;{ }^{13} \mathrm{C} \mathrm{NMR}\left(151 \mathrm{MHz}, \mathrm{CDCl}_{3}\right)$ : $\delta 171.7,141.5,137.0,136.6,131.2,128.9$ (2C) , $128.6(2 \mathrm{C}), 128.5(2 \mathrm{C}), 128.1(2 \mathrm{C}), 127.7(2 \mathrm{C}), 127.6,126.8$ , $126.1,125.6,50.4,50.0,47.6,35.7,33.0$; HRMS (ESI): calculated for $[\mathrm{M}+\mathrm{H}]^{+}\left(\mathrm{C}_{24} \mathrm{H}_{23} \mathrm{NO}\right) \mathrm{m} / \mathrm{z}: 342.1852$ found $[\mathrm{M}+\mathrm{H}]^{+}:$342.1854; IR (neat, $\mathrm{cm}^{-1}$ ): 3060, 3034, 2932, 1655, 1495, 1452. 

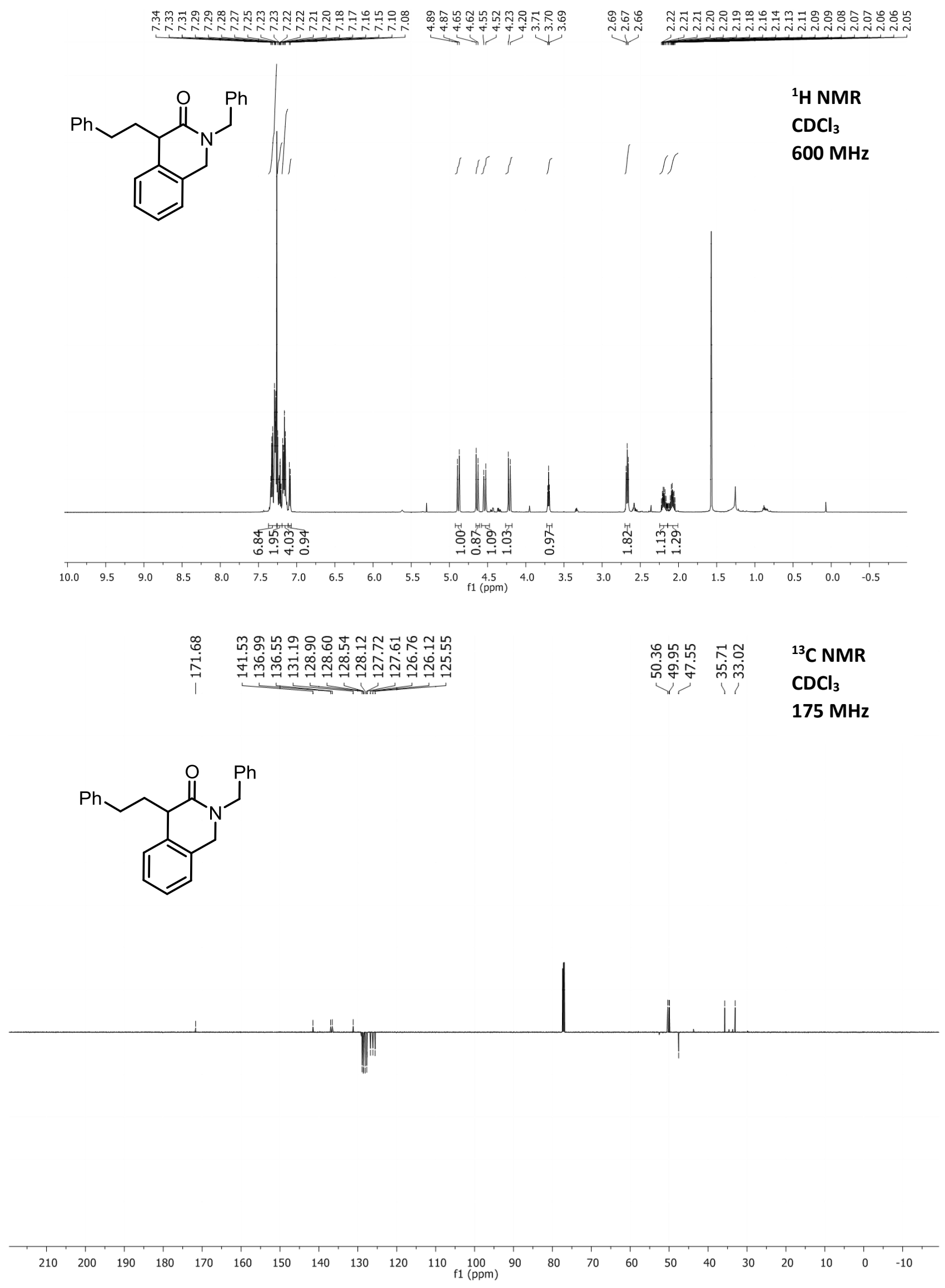


\section{Substitution on an independently formed $\alpha$-OTf amide.}
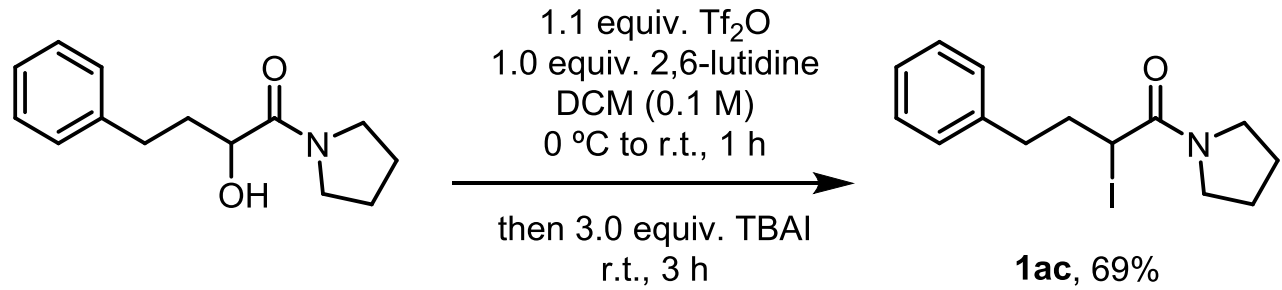

1 ac, $69 \%$

To a solution of the amide $2 \mathrm{~m}(0.2 \mathrm{mmol}, 1$ equiv.) and distilled 2,6-lutidine (1 equiv.) in DCM (0.1 M, $2 \mathrm{~mL})$ at $0{ }^{\circ} \mathrm{C}$, triflic anhydride ( $35 \mu \mathrm{L}, 1.1$ equiv.) was added dropwise and the resulting reaction mixture was allowed to warm to room temperature while stirring for 1 hour. TBAI $(0.6 \mathrm{mmol}, 3$ equiv.) was added and the reaction mixture was stirred at room temperature for $3 \mathrm{~h}$ before being quenched with $\mathrm{NH}_{4} \mathrm{Cl}$ solution. The layers were separated and the aqueous extracted with DCM. The combined organic layers were washed with brine before being dried over anhydrous $\mathrm{MgSO}_{4}$. The solvent was removed under reduced pressure. The crude product was purified by column chromatography (EtOAc in heptane 10\% -40\%) to afford the product as a yellow oil in $69 \%$ (47.1 mg) yield.

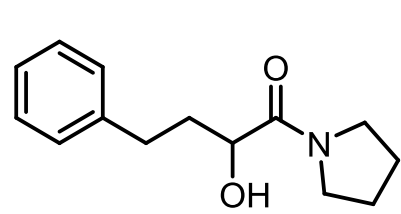

To a solution of the amide $2 \mathrm{~m}(0.2 \mathrm{mmol}, 1$ equiv.) and distilled 2,6-lutidine (1 equiv.) in DCM (0.1 M, $2 \mathrm{~mL})$ at $0{ }^{\circ} \mathrm{C}$, triflic anhydride (35 $\mu \mathrm{L}, 1.1$ equiv.) was added dropwise and the resulting reaction mixture was allowed to warm to room temperature while stirring for 1 hour. Then, a solution of the deprotonated nucleophile was generated from addition of the $\mathrm{N}$-Methyl-p-toluenesulfonamide $(0.6 \mathrm{mmol}, 3$ equiv.) to a suspension of $\mathrm{NaH}(0.6$ mmol, 3 equiv.) in DMF ( $3.0 \mathrm{~mL}$ ) was added. The reaction mixture was stirred at room temperature for $3 \mathrm{~h}$ before being quenched with $\mathrm{NH}_{4} \mathrm{Cl}$ solution. The layers were separated and the aqueous extracted with DCM. The combined organic layers were washed with brine before being dried over anhydrous $\mathrm{MgSO}_{4}$. The solvent was removed under reduced pressure. The crude product was purified by column chromatography (EtOAc in heptane $20 \%-40 \%)$ to afford the product as a yellow oil in $82 \%(65.9 \mathrm{mg})$ yield. 
Regarding the mode of addition of the sulfonamide

\begin{tabular}{|c|c|c|c|c|c|}
\hline & & & & $\begin{array}{l}2 \text { eq. } 2 \mathrm{l}-\mathrm{Py}, 1.1 \text { eq. } \mathrm{Tf}_{2} \mathrm{O} \\
\mathrm{CM}(0.1 \mathrm{M}), 0^{\circ} \mathrm{C}, 15 \mathrm{~min} \\
\stackrel{1 \text { eq. } \mathrm{LNO},}{\longrightarrow} \\
\text { then } 3 \text { eq. } \mathrm{HNMeNs} \\
\text { or NaNMeNs }\end{array}$ & \\
\hline entry & $\mathrm{T}\left({ }^{\circ} \mathrm{C}\right)$ & Time (h) & solvent & Nucleophile addition conditions & $\begin{array}{l}\text { NMR } \\
\text { yield }^{\text {a }}\end{array}$ \\
\hline 1 & r.t. & 1 & DCM & no base & 0 \\
\hline 2 & r.t. & 1 & DCM & $\mathrm{NaH}+$ sulfonamide added directly & 0 \\
\hline 3 & r.t. & 1 & DCM & $\mathrm{Cs}_{2} \mathrm{CO}_{3}+$ sulfonamide added directly & 0 \\
\hline 4 & r.t. & 1 & DCM & no base + TBAHFP & 0 \\
\hline 5 & r.t. & 1 & $\mathrm{MeCN}$ & $\begin{array}{c}\mathrm{NaH}+\text { sulfonamide added as a } \mathrm{MeCN} \\
\text { suspension }\end{array}$ & 37 \\
\hline 6 & r.t. & 1 & $\mathrm{MeCN}$ & direct addition of $\mathrm{Na}$-sulfonamidate & 44 \\
\hline 7 & r.t. & 20 & $\mathrm{MeCN}$ & direct addition of Na-sulfonamidate & 43 \\
\hline 8 & r.t. & 2 & $\mathrm{MeCN}$ & KHMDS + sulfonamide added directly & 25 \\
\hline 9 & r.t. & 2 & $\mathrm{MeCN}$ & Na-sulfonamidate dissolved in $0.5 \mathrm{~mL}$ DMF & 45 \\
\hline 10 & 80 & 2 & $\mathrm{MeCN}$ & direct addition of Na-sulfonamidate salt & 31 \\
\hline
\end{tabular}

\section{Attempts towards an Umpolung - Smiles rearrangement one pot reaction}

\begin{tabular}{|c|c|c|c|c|c|}
\hline & & $\begin{array}{c}\text { 1) } \alpha \text {-amination } \\
\text { (procedure } E \text { modified) }\end{array}$ & O & $\begin{array}{r}1 \\
7 a\end{array}$ & \\
\hline entry & solvent & Step 1 & Step 2 & $\begin{array}{c}\text { NMR } \\
\text { yield } \mathbf{4} \mathbf{f}^{\mathrm{a}}\end{array}$ & $\begin{array}{c}N M R \\
\text { yield } 7 a^{a}\end{array}$ \\
\hline 1 & $\mathrm{MeCN}$ & $\begin{array}{c}\mathrm{NaH}+\text { sulfonamide } \\
\text { supended in } \mathrm{MeCN}(0.12 \mathrm{M})\end{array}$ & 3 eq. $\mathrm{Pr}_{4} \mathrm{NOH}$ & 32 & 0 \\
\hline 2 & $\mathrm{MeCN}$ & Na-sulfonamidate & 10 eq. $\mathrm{Pr}_{4} \mathrm{NOH}$ & 4 & 19 \\
\hline 3 & $\mathrm{MeCN}$ & $\begin{array}{c}\text { Na-sulfonamidate }+2 \text { eq. } \\
\text { TBAHFP }\end{array}$ & 10 eq. $\mathrm{Pr}_{4} \mathrm{NOH}$ & 1 & 15 \\
\hline 4 & DCM & $\begin{array}{l}\mathrm{NaH}+\text { sulfonamide in DMF } \\
(0.6 \mathrm{M})\end{array}$ & $\begin{array}{c}\text { Addition of } 3 \mathrm{~mL} \mathrm{MeCN} \text {, } \\
10 \text { eq. } \mathrm{Bu}_{4} \mathrm{NOH}\end{array}$ & 70 & 0 \\
\hline 5 & DCM & $\begin{array}{c}\mathrm{NaH}+\text { sulfonamide in DMF } \\
(0.6 \mathrm{M})\end{array}$ & $\begin{array}{c}\text { Addition of } 3 \mathrm{~mL} \\
\text { dioxane, } 10 \text { eq. } \mathrm{Bu}_{4} \mathrm{NOH}\end{array}$ & 69 & 0 \\
\hline
\end{tabular}

a Using 1,3,5-trimethoxybenzene as an internal standard in ${ }^{1} \mathrm{H}$ NMR. 


\section{General procedures for the synthesis of carboxamides and sulfonamides}

\section{Carboxamides - Procedure A}

To a solution of the amine (1.0 equiv.) and triethylamine (2.0 equiv.) in $\mathrm{DCM}(0.1 \mathrm{M})$ at $0{ }^{\circ} \mathrm{C}$, the corresponding acyl chloride (1.2 equiv.) was added dropwise and the resulting reaction mixture was allowed to warm to room temperature while stirring overnight $(14 \mathrm{~h})$. After this time, a saturated aqueous solution of sodium bicarbonate was added and the biphasic system was separated. The aqueous phase was extracted with DCM (1x) and the organic phases were combined and dried over anhydrous sodium sulfate. The dried solution was filtered and concentrated under reduced pressure. The resulting crude material was purified by flash column chromatography on silica gel (heptane/ethyl acetate) to afford the desired compound.

\section{Carboxamides - Procedure B}

To a solution of the amine (1.0 equiv.), triethylamine (1.0 equiv.), hydroxybenzotriazole (HOBt, 1.0 equiv.) and 1-ethyl-3-(3-dimethylaminopropyl)carbodiimide hydrochloride $(\mathrm{EDCl} * \mathrm{HCl}, 1.0$ equiv.) in $\mathrm{DCM}(0.1 \mathrm{M})$, the corresponding carboxylic acid was added and the resulting solution was stirred at room temperature overnight $(14 \mathrm{~h})$. After this time, the organic solution was extracted sequentially with $0.5 \mathrm{M}$ aqueous hydrochloric acid, saturated aqueous sodium bicarbonate and saturated aqueous sodium chloride. The washed solution was dried over anhydrous sodium sulfate, filtered and concentrated under reduced pressure. The resulting crude material was purified by flash column chromatography on silica gel (heptane/ethyl acetate) to afford the desired compound.

\section{Sulfonamides - Procedure C}

To a solution of sulfonyl chloride (1.0 equiv.) in THF $(1 \mathrm{M})$ was added the primary amine (3.0 equiv.) or the primary amine hydrochloride salt (1.0 equiv.) in combination with triethylamine ( 2.5 equiv.) at room temperature. The mixture was stirred for $16 \mathrm{~h}$ while a precipitate formed. Then a solution of $\mathrm{HCl}(1 \mathrm{M})$ was added until a $\mathrm{pH}$ of 2 was reached. The solution was extracted with EtOAc. The combined organic layers were washed with a saturated solution of $\mathrm{NaCl}$ and dried over $\mathrm{MgSO}_{4}$. The solvent was removed under reduced pressure which yielded the product. The crude product was used without further purification. 
4-Phenyl-1-(pyrrolidin-1-yl)butan-1-one<smiles>O=C(CCCc1ccccc1)N1CCCC1</smiles>

The product was prepared according to general procedure B from $2.46 \mathrm{~g}(15.0 \mathrm{mmol})$ 4-phenylbutyric acid and $1.07 \mathrm{~g}(1.24 \mathrm{~mL}, 15.0 \mathrm{mmol})$ pyrrolidine in 93\% (3.04 g) yield. All spectroscopic properties are in good accordance with reported data.[1]

\section{$N, N$-dimethyl-4-phenylbutanamide}<smiles>CN(C)C(=O)CCCc1ccccc1</smiles>

The product was prepared according to general procedure B from $2.46 \mathrm{~g}(15.0 \mathrm{mmol})$ 4-phenylbutyric acid and 4-nitrobenzenesulfonyl chloride and $7.5 \mathrm{~mL}(15.0 \mathrm{mmol})$ dimethylamine $(2.0 \mathrm{M}$ in THF) in $71 \%$ (2.04 g) yield. All spectroscopic properties are in good accordance with reported data. [2]

\section{6-chloro-1-(pyrrolidin-1-yl)hexan-1-one}<smiles>O=C(CCCCCCl)N1CCCC1</smiles>

The product was prepared according to general procedure A from $507 \mathrm{mg}$ (3.0 mmol) 6-chlorohexanoyl chloride and $213 \mathrm{mg}(0.25 \mathrm{~mL}, 3 \mathrm{mmol})$ pyrrolidine in $98 \%(600 \mathrm{mg})$ yield. All spectroscopic properties are in good accordance with reported data.[1]

\section{Methyl 9-oxo-9-(pyrrolidin-1-yl)nonanoate}<smiles>COC(=O)CCCCCCCC(=O)N1CCCC1</smiles> 
The product was prepared according to general procedure B from $1.42 \mathrm{~g}$ (7.0 mmol) monomethyl azelate and $0.50 \mathrm{~g}(0.57 \mathrm{~mL}, 7.0 \mathrm{mmol})$ pyrrolidine in $99 \%$ (1.77 g) yield. All spectroscopic properties are in good accordance with reported data.[1]

\section{1-(Pyrrolidin-1-yl)undecane-1,10-dione}<smiles>CC(=O)CCCCCCCCC(=O)N1CCCC1</smiles>

Prepared according to the procedure reported in the literature.[3] All analytical data were in good accordance with data reported in the literature.

\section{1-(pyrrolidin-1-yl)undec-10-en-1-one}<smiles>C=CCCCCCCCCC(=O)N1CCCC1</smiles>

The product was prepared according to general procedure A from $1.22 \mathrm{~g}(6.0 \mathrm{mmol}) 10$-undecenoyl chloride and $866 \mathrm{mg}(1.0 \mathrm{~mL}, 5.0 \mathrm{mmol})$ pyrrolidine in $99 \%$ (1.19 g) yield. All spectroscopic properties are in good accordance with reported data.[4]

\section{1-(Pyrrolidin-1-yl)hex-5-yn-1-one}<smiles>C#CCCCC(=O)N1CCCC1</smiles>

The product was prepared according to general procedure B from $135 \mathrm{mg}$ ( $1.2 \mathrm{mmol}) 5$-hexynoic acid and $85 \mathrm{mg}$ $(0.1 \mathrm{~mL}, 1.2 \mathrm{mmol})$ pyrrolidine in $96 \%$ (190 mg) yield. All spectroscopic properties are in good accordance with reported data.[5] 


\section{7-0xo-7-(pyrrolidin-1-yl)heptanenitrile}<smiles>N#CCCCCCC(=O)N1CCCC1</smiles>

Prepared according to the procedure reported in the literature.[3] All analytical data were in good accordance with data reported in the literature.

\section{3-Phenyl-1-(pyrrolidin-1-yl)propan-1-one}<smiles>O=C(CCc1ccccc1)N1CCCC1</smiles>

The product was prepared according to general procedure A from $674 \mathrm{mg}$ (4.0 mmol) hydrocinnamoyl chloride and $341 \mathrm{mg}(0.4 \mathrm{~mL}, 4.8 \mathrm{mmol})$ pyrrolidine in $99 \%(810 \mathrm{mg}$ ) yield. All spectroscopic properties are in good accordance with reported data.[6]

\section{1-(Azepan-1-yl)-4-phenylbutan-1-one}<smiles>O=C(CCCc1ccccc1)N1CCCCCC1</smiles>

The product was prepared according to general procedure B from $821 \mathrm{mg}(5.0 \mathrm{mmol})$ 4-phenylbutyric acid and $496 \mathrm{mg}$ ( $546 \mu \mathrm{L}, 5.0 \mathrm{mmol}$ ) hexamethyleneimine. Purification by column chromatography on silica gel (20-50\% EtOAc:heptane) yielded the product $(1.07 \mathrm{~g}, 88 \%)$ as a pale yellow oil.

${ }^{1} \mathrm{H}$ NMR $\left(400 \mathrm{MHz}, \mathrm{CDCl}_{3}\right): \delta=7.30-7.25(\mathrm{~m}, 2 \mathrm{H}), 7.21-7.15(\mathrm{~m}, 3 \mathrm{H}), 3.52(\mathrm{t}, J=6.0 \mathrm{~Hz}, 2 \mathrm{H}), 3.35(\mathrm{t}, J=6.0 \mathrm{~Hz}$, $2 \mathrm{H}), 2.68(\mathrm{t}, J=7.5 \mathrm{~Hz}, 2 \mathrm{H}), 2.32(\mathrm{t}, J=7.2 \mathrm{~Hz}, 2 \mathrm{H}), 2.06-1.95(\mathrm{~m}, 2 \mathrm{H}), 1.74-1.62(\mathrm{~m}, 4 \mathrm{H}), 1.59-1.51(\mathrm{~m}, 4 \mathrm{H})$; ${ }^{13} \mathrm{C}$ NMR (100 MHz, CDCl $): \delta=172.4,142.1,128.7$ (2C), 128.5 (2C), 126.0, 48.0, 46.1, 35.6, 32.5, 29.3, 27.8, 27.2, 27.0, 26.9; HRMS (ESI): calculated for $[\mathrm{M}+\mathrm{Na}]^{+}\left(\mathrm{C}_{16} \mathrm{H}_{23} \mathrm{NNaO}\right)$ 268.1672, found 268.1684; IR (neat, $\left.\mathrm{cm}^{-1}\right):=2925$, $2854,1635,1425,1262,1194,746,700$. 


\section{N-Methyl-4-nitrobenzenesulfonamide}<smiles>CNS(=O)(=O)c1ccc([N+](=O)[O-])cc1</smiles>

The product was prepared according to general procedure $C$ from $2.22 \mathrm{~g} \mathrm{(10.0} \mathrm{mmol)} \mathrm{4-nitrobenzenesulfonyl}$ chloride and $15.0 \mathrm{~mL}$ (30.0 mmol) methylamine (2.0 M in THF) in 96\% (2.12 g) yield. All spectroscopic properties are in good accordance with reported data.[7]

\section{N-Isopropyl-4-methylbenzenesulfonamide}

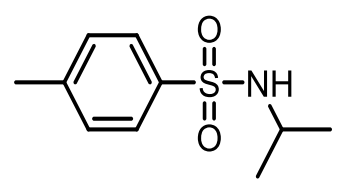

The product was prepared according to general procedure C from $381 \mathrm{mg}$ ( $2.0 \mathrm{mmol})$ 4-methylbenzenesulfonyl chloride and $355 \mathrm{mg}(0.49 \mathrm{~mL}, 6.0 \mathrm{mmol})$ iso-propylamine in 95\% (404 mg) yield. All spectroscopic properties are in good accordance with reported data.[8]

\section{N-Benzyl-4-methylbenzenesulfonamide}

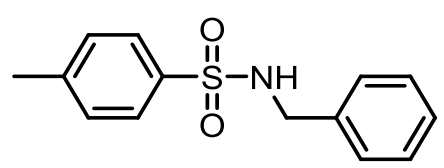

The product was prepared according to general procedure $C$ from $381 \mathrm{mg}(2.0 \mathrm{mmol})$ 4-methylbenzenesulfonyl chloride and $643 \mathrm{mg}(0.65 \mathrm{~mL}, 6.0 \mathrm{mmol}$ ) benzylamine in $94 \%$ (489 mg) yield. All spectroscopic properties are in good accordance with reported data.[9]

\section{4-Methyl-N-phenylbenzenesulfonamide}<smiles>Cc1ccc(S(=O)(=O)Nc2ccccc2)cc1</smiles>

The product was prepared according to general procedure C from $381 \mathrm{mg}(2.0 \mathrm{mmol})$ 4-methylbenzenesulfonyl chloride and $559 \mathrm{mg}(0.55 \mathrm{~mL}, 6.0 \mathrm{mmol})$ aniline in $87 \%(432 \mathrm{mg})$ yield. All spectroscopic properties are in good accordance with reported data.[9] 


\section{$\mathrm{N}$-(4-methoxyphenyl)-4-methylbenzenesulfonamide}

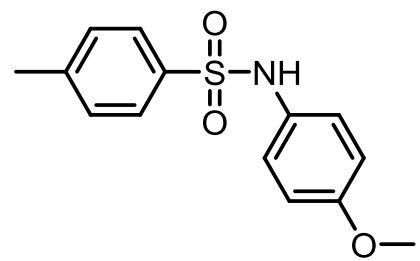

The product was prepared according to general procedure C from $4.58 \mathrm{~g}$ ( $24.0 \mathrm{mmol}) 4$-methylbenzenesulfonyl chloride, $2.46 \mathrm{~g}(20.0 \mathrm{mmol})$ 4-methoxyaniline $2.43 \mathrm{~g}(24.0 \mathrm{mmol})$ triethylamine in $89 \%$ (4.93 g) yield. All spectroscopic properties are in good accordance with reported data.[9]

\section{Methyl tosylglycinate}<smiles>COC(=O)CNS(=O)(=O)c1ccc(C)cc1</smiles>

The product was prepared according to general procedure C from $381 \mathrm{mg}$ ( $2.0 \mathrm{mmol})$ 4-methylbenzenesulfonyl chloride, $281 \mathrm{mg}(2.0 \mathrm{mmol})$ glycine methyl ester hydrochloride and $506 \mathrm{mg}(0.76 \mathrm{~mL}, 5 \mathrm{mmol})$ trimethylamine in 91\% (445 mg) yield. All spectroscopic properties are in good accordance with reported data.[10]

\section{N-Methyl-2-nitrobenzenesulfonamide}

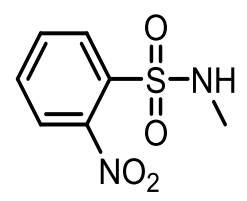

The product was prepared according to general procedure $C$ from $665 \mathrm{mg}$ (3.0 mmol) 2-nitrobenzenesulfonyl chloride and $4.5 \mathrm{~mL}(9.0 \mathrm{mmol})$ methylamine $(2.0 \mathrm{M}$ in THF) in $93 \%$ (600 mg) yield. All spectroscopic properties are in good accordance with reported data.[11]

\section{N-Methylnaphthalene-2-sulfonamide}<smiles>CNS(=O)(=O)c1ccc2ccccc2c1</smiles> 
The product was prepared according to general procedure $C$ from $453 \mathrm{mg}$ ( $2.0 \mathrm{mmol}$ ) naphthalene-2-sulfonyl chloride and $3.0 \mathrm{~mL}(6.0 \mathrm{mmol})$ methylamine $(2.0 \mathrm{M}$ in THF) in $96 \%$ (423 mg) yield. All spectroscopic properties are in good accordance with reported data.[12]

\section{N-Methylpyridine-2-sulfonamide}<smiles>CNS(=O)(=O)c1ccccn1</smiles>

2-Mercaptopyridine $(0.561 \mathrm{~g}, 5.0 \mathrm{mmol})$ was stirred in a mixture of $25.0 \mathrm{~mL}$ of DCM and $25.0 \mathrm{~mL}$ of a $1.0 \mathrm{M}$ solution of $\mathrm{HCl}$ for $10 \mathrm{~min}$ at -10 to $-5{ }^{\circ} \mathrm{C}$. Then, a cold $\left(5{ }^{\circ} \mathrm{C}\right)$ solution of sodium hypochlorite (11\% (aq.), $9.3 \mathrm{~mL}$, $16.5 \mathrm{mmol}$ ) was added dropwise under vigorous stirring. After the addition was completed, the mixture was stirred for $15 \mathrm{~min}$ from -10 to $-5^{\circ} \mathrm{C}$. It was transferred to a separatory funnel (pre-cooled in the freezer) and the organic layer was rapidly separated and collected in an Erlenmeyer flask cooled in a dry ice-acetone bath. Methylamine (2.0 M in THF, $6.25 \mathrm{~mL}, 12.5 \mathrm{mmol}$ ) was added while stirring, whereupon the organic layer became a white suspension. The suspension was stirred for $30 \mathrm{~min}$ at $0{ }^{\circ} \mathrm{C}$. Then, the mixture was washed with $1.0 \mathrm{M}$ solution of $\mathrm{HCl}$, water and a solution of saturated $\mathrm{NaCl}$, dried with $\mathrm{MgSO}_{4}$ and concentrated under reduced pressure. Purification by column chromatography on silica gel (EtOAc:heptane $=1: 1$ ) yielded the product as a white solid (400 mg, 47\%).[13]

${ }^{1} \mathrm{H}$ NMR $\left(400 \mathrm{MHz}, \mathrm{CDCl}_{3}\right): \delta=8.75-8.69(\mathrm{~m}, 1 \mathrm{H}), 8.02(\mathrm{dt}, J=7.7,0.9 \mathrm{~Hz}, 1 \mathrm{H}), 7.93(\mathrm{td}, J=7.7,1.7 \mathrm{~Hz}, 1 \mathrm{H}), 7.51$ (ddd, $J=7.6,4.7,1.2 \mathrm{~Hz}, 1 \mathrm{H}$ ), 4.94 (s.br, 1H), 2.75 (d, $J=5.3 \mathrm{~Hz}, 3 \mathrm{H}) ;{ }^{13} \mathrm{C}$ NMR (175 MHz, $\mathrm{CDCl}_{3}$ ): $\delta=156.7,150.2$, 138.2 , 126.9 , 122.8 , 29.9; HRMS (ESI): calculated for $[\mathrm{M}+\mathrm{Na}]^{+}\left(\mathrm{C}_{6} \mathrm{H}_{8} \mathrm{~N}_{2} \mathrm{O}_{2} \mathrm{SNa}\right) \mathrm{m} / \mathrm{z}$ : 195.0199, found 195.0198; IR (neat, cm cm $^{-1}$ : 3289, 1579, 1454, 1428, 1325, 1174, 1117, 1086, 992, 844, 777, 738, 590.

\section{$\mathrm{N}, \mathrm{N}$-dibenzyl-4-phenylbutanamide}<smiles>O=C(CCCc1ccccc1)N(Cc1ccccc1)Cc1ccccc1</smiles>

The product was prepared according to general procedure A. Purification by flash column chromatography yielded the product as a yellow oil (84\%). 
${ }^{1} \mathrm{H}$ NMR $\left(600 \mathrm{MHz}, \mathrm{CDCl}_{3}\right): \delta 7.35(\mathrm{t}, J=7.4 \mathrm{~Hz}, 2 \mathrm{H}), 7.33-7.26(\mathrm{~m}, 4 \mathrm{H}), 7.25(\mathrm{~d}, J=7.6 \mathrm{~Hz}, 2 \mathrm{H}), 7.22(\mathrm{~d}, J=7.3$ $\mathrm{Hz}, 2 \mathrm{H}), 7.18-7.15(\mathrm{~m}, 3 \mathrm{H}), 7.10(\mathrm{~d}, J=7.4 \mathrm{~Hz}, 2 \mathrm{H}), 4.61(\mathrm{~s}, 2 \mathrm{H}), 4.38(\mathrm{~s}, 2 \mathrm{H}), 2.68(\mathrm{t}, J=7.6 \mathrm{~Hz}, 2 \mathrm{H}), 2.43(\mathrm{t}, J=$ $7.4 \mathrm{~Hz}, 2 \mathrm{H}), 2.08-2.02(\mathrm{~m}, 2 \mathrm{H}) ;{ }^{13} \mathrm{C}$ NMR (151 MHz, CDCl 3$): \delta 173.4,141.8,137.7,136.8,129.1(2 \mathrm{C}), 128.7$ (2C) , $128.6(2 \mathrm{C}), 128.5(2 \mathrm{C}), 128.4(2 \mathrm{C}), 127.7$ (2C) , $127.5,126.5,126.0,50.0,48.3$, 35.4 , 32.6 , 27.0; HRMS (ESI): calculated for $[\mathrm{M}+\mathrm{Na}]^{+}\left(\mathrm{C}_{24} \mathrm{H}_{25} \mathrm{NONa}\right) \mathrm{m} / \mathrm{z}: 366.1834$ found $[\mathrm{M}+\mathrm{Na}]^{+}: 366.1823$; IR (neat, $\left.\mathbf{c m}^{-1}\right): 3062,3032$, $2928,1644,1494,1452$. 

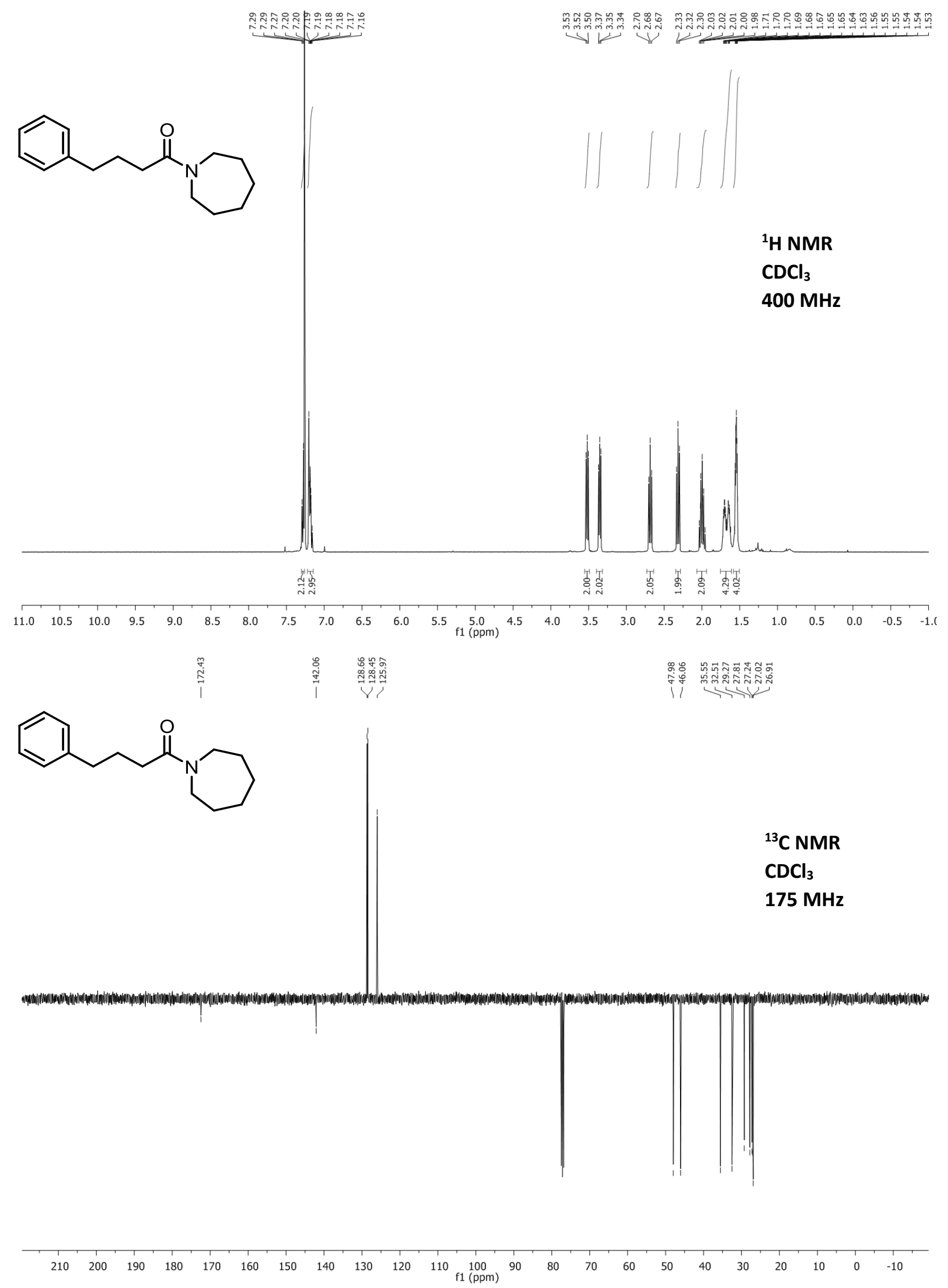


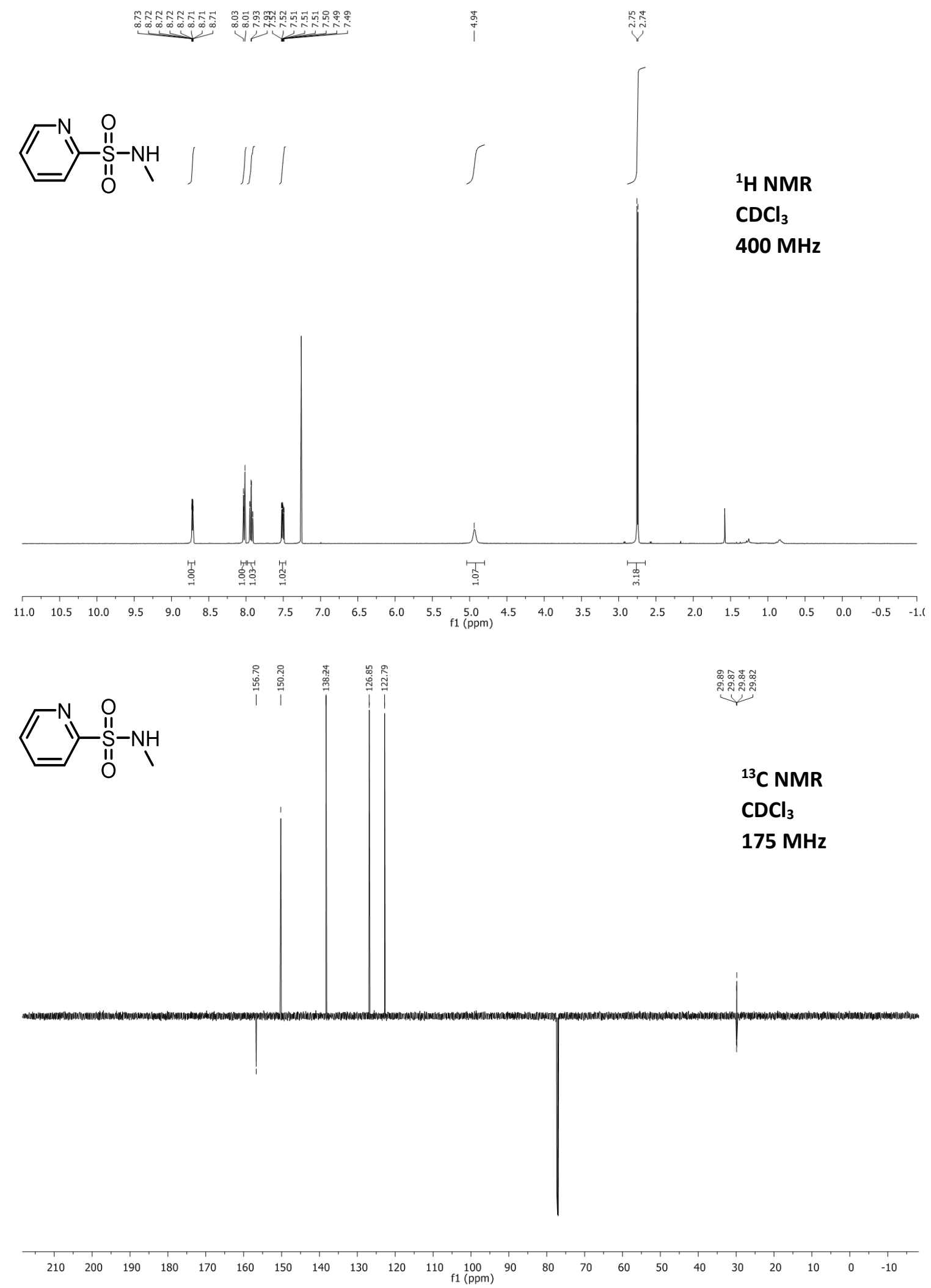



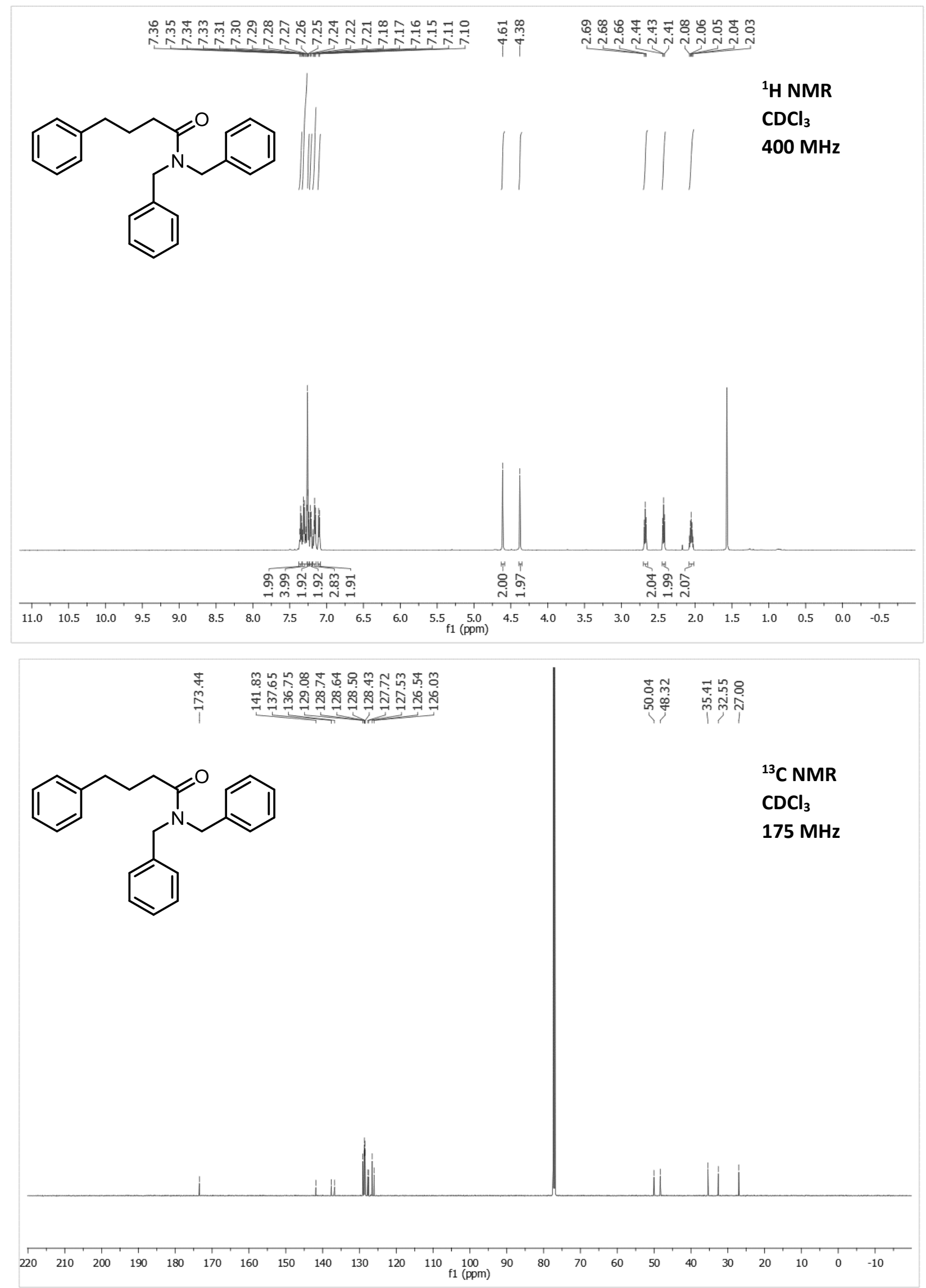


\section{General procedures for the $\alpha$-functionalization of amides}

\section{Halogens - Procedure D}

To a mixture of amide $(0.20 \mathrm{mmol})$, 2-iodopyridine $(0.44 \mathrm{mmol})$ in DCM $(2.0 \mathrm{~mL})$ was added triflic anhydride (37 $\mu \mathrm{L}, 0.22 \mathrm{mmol})$ dropwise under $\operatorname{Ar}$ at $0{ }^{\circ} \mathrm{C}$. After stirring for $15 \mathrm{~min}$ at $0{ }^{\circ} \mathrm{C}, 2,6$-lutidine $N$-oxide $(22.4 \mu \mathrm{L}, 0.20$ $\mathrm{mmol}$, ) was added and the reaction stirred for a further $5 \mathrm{~min}$ at $0{ }^{\circ} \mathrm{C}$. Then a tetrabutylammonium salt of the halogen was added in one portion (3 equiv.). The reaction mixture was stirred at room temperature for $3 \mathrm{~h}$ before being quenched with $\mathrm{NH}_{4} \mathrm{Cl}$ solution. The layers were separated and the aqueous extracted with DCM. The combined organic layers were washed with brine before being dried over anhydrous $\mathrm{MgSO}_{4}$. The solvent was removed under reduced pressure.

\section{$\mathrm{O}-, \mathrm{S}$ - and $\mathrm{N}$-Nucleophiles - Procedure $\mathrm{E}$}

To a mixture of amide $(0.20 \mathrm{mmol})$, 2-iodopyridine $(0.44 \mathrm{mmol})$ in DCM $(2.0 \mathrm{~mL})$ was added triflic anhydride (37 $\mu \mathrm{L}, 0.22 \mathrm{mmol})$ dropwise under $\mathrm{Ar}$ at $0{ }^{\circ} \mathrm{C}$. After stirring for $15 \mathrm{~min}, 2,6$-lutidine $\mathrm{N}$-oxide $(22.4 \mu \mathrm{L}, 0.20 \mathrm{mmol}$, $)$ was added and the reaction stirred for a further $5 \mathrm{~min}$ at $0{ }^{\circ} \mathrm{C}$. Then, a solution of the deprotonated nucleophile was generated from addition of the nucleophile $(0.60 \mathrm{mmol})$ to a suspension of $\mathrm{NaH}(0.60 \mathrm{mmol})$ in DMF $(3.0$ $\mathrm{mL}$ ) was added. The reaction mixture was stirred at room temperature for $3 \mathrm{~h}$ before being quenched with $\mathrm{NH}_{4} \mathrm{Cl}$ solution. The layers were separated and the aqueous extracted with DCM. The combined organic layers were washed with brine before being dried over anhydrous $\mathrm{MgSO}_{4}$. The solvent was removed under reduced pressure.

\section{$\alpha$-Arylation via Smiles rearrangement - Procedure F}

To a solution of the amide (1 equiv.) in MeCN (0.1 M) was added tetrapropyl ammonium hydroxide (40\% (aq.), 2 eq.) The solution was stirred for $16 \mathrm{~h}$. Then it was washed with saturated $\mathrm{NH}_{4} \mathrm{Cl}$ solution and brine. The organic layer was dried with $\mathrm{MgSO}_{4}$, the solvent evaporated under reduced pressure and the crude product purified by column chromatography on silica gel. 
1aa: 2-Chloro-4-phenyl-1-(pyrrolidin-1-yl)butan-1-one<smiles>O=C(C(Cl)CCc1ccccc1)N1CCCC1</smiles>

The product was prepared according to general procedure D. Purification by flash column chromatography (EtOAc: heptane = 1:4-1:1) yielded the product as a yellow oil (42.0 mg, 84\% / 10mmol scale - $1.85 \mathrm{~g}, 75 \%$ ).

When $\mathrm{NaCl}(95 \%)$ was used as a halide source the reaction was stirred for 20 hours instead and yielded the product in $90 \%$ (45.1 mg).

${ }^{1} \mathrm{H}$ NMR $\left(700 \mathrm{MHz}, \mathrm{CDCl}_{3}\right): \delta=7.29(\mathrm{t}, J=7.5 \mathrm{~Hz}, 2 \mathrm{H}), 7.21-7.17(\mathrm{~m}, 3 \mathrm{H}), 4.14(\mathrm{t}, J=7.3 \mathrm{~Hz}, 1 \mathrm{H}), 3.52-3.46(\mathrm{~m}$, $3 \mathrm{H}), 3.14(\mathrm{dt}, J=9.8,7.0 \mathrm{~Hz}, 1 \mathrm{H}), 2.76(\mathrm{t}, J=7.3 \mathrm{~Hz}, 2 \mathrm{H}), 2.47-2.45(\mathrm{~m}, 1 \mathrm{H}), 2.37-2.35(\mathrm{~m}, 1 \mathrm{H}), 1.95-1.93(\mathrm{~m}$, 2H), $1.88-1.85$ (m, 2H); ${ }^{13} \mathrm{C}$ NMR (176 MHz, $\left.\mathrm{CDCl}_{3}\right)$ : $\delta=167.0,140.4,128.7$ (2C) , 128.6 (2C) , $126.4,46.5$ (2C) , 44.9 , 36.0 , 33.4 , 26.1 , 24.3; HRMS (ESI): calculated for [M+Na] ${ }^{+}\left(\mathrm{C}_{14} \mathrm{H}_{18} \mathrm{CINONa}\right) \mathrm{m} / \mathrm{z}: 274.0969$ found [M+Na] $]^{+}$: 274.0971; IR (neat, cm ${ }^{-1}$ ): 3025, 2950, 2874, 1643, 1495, 1433, 1340, 1189.

1ab: 2-Bromo-4-phenyl-1-(pyrrolidin-1-yl)butan-1-one<smiles>O=C(CCc1ccccc1)N1CCCC1</smiles>

The product was prepared according to general procedure D. Purification by flash column chromatography (EtOAc: heptane $=1: 4-1: 1$ ) yielded the product as a yellow oil $(44.2 \mathrm{mg}, 75 \%)$.

${ }^{1} \mathrm{H}$ NMR $\left(400 \mathrm{MHz}, \mathrm{CDCl}_{3}\right): \delta=7.31-7.26(\mathrm{~m}, 2 \mathrm{H}), 7.22-7.18(\mathrm{~m}, 3 \mathrm{H}), 4.18(\mathrm{dd}, J=7.9,6.4 \mathrm{~Hz}, 1 \mathrm{H}), 3.55-3.47$ $(\mathrm{m}, 3 \mathrm{H}), 3.21-3.18(\mathrm{~m}, 1 \mathrm{H}), 2.80-2.77(\mathrm{~m}, 2 \mathrm{H}), 2.39-2.36(\mathrm{~m}, 1 \mathrm{H}), 2.30-2.27(\mathrm{~m}, 1 \mathrm{H}), 1.95-1.83(\mathrm{~m}, 4 \mathrm{H}) ;{ }^{13} \mathrm{C}$ NMR (176 MHz, $\mathrm{CDCl}_{3}$ ): $\delta=166.9,140.5,128.7$ (2C) , 128.7 (2C) , $126.4,55.0,46.5,46.4$, 35.6 , 32.3 , 26.2 ， 24.3; HRMS (ESI): calculated for $[\mathrm{M}+\mathrm{Na}]^{+}\left(\mathrm{C}_{14} \mathrm{H}_{18} \mathrm{BrNONa}\right) \mathrm{m} / \mathrm{z}$ : 318.0464 found $[\mathrm{M}+\mathrm{Na}]^{+}$: 318.0462; IR (neat, $\mathrm{cm}^{-}$ $\left.{ }^{1}\right): 2972,2875,1651,1438,752,700$.

1ac: 2-lodo-4-phenyl-1-(pyrrolidin-1-yl)butan-1-one<smiles>O=C(C(I)CCc1ccccc1)N1CCCC1</smiles> 
The product was prepared according to general procedure D. Purification by flash column chromatography (EtOAc: heptane $=1: 4-1: 1$ ) yielded the product as a yellow oil $(58.5 \mathrm{mg}, 80 \%)$.

${ }^{1} \mathrm{H}$ NMR $\left(600 \mathrm{MHz}, \mathrm{CDCl}_{3}\right): \delta=7.30-7.28(\mathrm{~m}, 2 \mathrm{H}), 7.21(\mathrm{t}, J=7.3 \mathrm{~Hz}, 1 \mathrm{H}), 7.17(\mathrm{~d}, J=7.4 \mathrm{~Hz}, 2 \mathrm{H}), 4.15(\mathrm{t}, J=7.4$ $\mathrm{Hz}, 1 \mathrm{H}), 3.46(\mathrm{t}, J=6.9 \mathrm{~Hz}, 2 \mathrm{H}), 3.35(\mathrm{dt}, J=9.7,6.7 \mathrm{~Hz}, 1 \mathrm{H}), 3.05-3.01(\mathrm{~m}, 1 \mathrm{H}), 2.73-2.69(\mathrm{~m}, 2 \mathrm{H}), 2.44(\mathrm{td}, J=$ 14.2, 7.5 Hz, 1H), $2.36(\mathrm{td}, J=14.5,7.3 \mathrm{~Hz}, 1 \mathrm{H}), 1.98-1.91(\mathrm{~m}, 2 \mathrm{H}), 1.89-1.81(\mathrm{~m}, 2 \mathrm{H}) ;{ }^{13} \mathrm{C} \mathrm{NMR}(151 \mathrm{MHz}$, $\mathrm{CDCl}_{3}$ ): $\delta=168.5$, $140.3,128.7$ (2C) , 128.7 (2C) , $126.4,46.8$, $46.7,37.7$, 35.2 , 26.1 , 24.4 , 22.3; HRMS (ESI): calculated for $[\mathrm{M}+\mathrm{Na}]^{+}\left(\mathrm{C}_{14} \mathrm{H}_{18} \mathrm{INONa}\right) \mathrm{m} / \mathrm{z}: 366.0325$ found $[\mathrm{M}+\mathrm{Na}]^{+}: 366.0331$; IR (neat, $\left.\mathbf{c m}^{-1}\right): 2969,2928,2873$, $1642,1430,1341,750,700$.

\section{1ba: 2-Chloro-N,N-dimethyl-4-phenylbutanamide}<smiles>CN(C)C(=O)C(Cl)CCc1ccccc1</smiles>

The product was prepared according to general procedure D. Purification by flash column chromatography (EtOAc: heptane $=1: 4-2: 1$ ) yielded the product as a yellow oil $(38.7 \mathrm{mg}, 86 \%)$.

${ }^{1} \mathrm{H}$ NMR $\left(600 \mathrm{MHz}, \mathrm{CDCl}_{3}\right): \delta=7.31-7.28(\mathrm{~m}, 2 \mathrm{H}), 7.22-7.18(\mathrm{~m}, 3 \mathrm{H}), 4.32(\mathrm{t}, J=7.0 \mathrm{~Hz}, 1 \mathrm{H}), 2.98(\mathrm{~s}, 3 \mathrm{H}), 2.97$ $(\mathrm{s}, 3 \mathrm{H}), 2.78(\mathrm{t}, J=7.3 \mathrm{~Hz}, 2 \mathrm{H}), 2.38-2.26(\mathrm{~m}, 2 \mathrm{H}) ;{ }^{13} \mathrm{C}$ NMR (151 MHz, CDCl 3$): \delta=168.4,140.5,128.7(2 \mathrm{C})$, 128.6 (2C) , $126.4,53.0,37.3,36.3$, $35.8,32.3$; HRMS (ESI): calculated for $[\mathrm{M}+\mathrm{Na}]^{+}\left(\mathrm{C}_{12} \mathrm{H}_{16} \mathrm{CINONa}\right) \mathrm{m} / \mathrm{z}$ : 248.0818 found [M+Na] $]^{+}$248.0812; IR (neat, $\mathrm{cm}^{-1}$ ): 2933, 2867, 1656, 1495, 1545, 1401.

\section{1bb: 2-Bromo-N,N-dimethyl-4-phenylbutanamide}<smiles>CN(C)C(=O)C(Br)CCc1ccccc1</smiles>

The product was prepared according to general procedure D. Purification by flash column chromatography (EtOAc: heptane = 1:4-2:1) yielded the product as a yellow oil $(49.5 \mathrm{mg}, 92 \%)$.

${ }^{1} \mathrm{H}$ NMR $\left(600 \mathrm{MHz}, \mathrm{CDCl}_{3}\right): \delta=7.31-7.29(\mathrm{~m}, 2 \mathrm{H}), 7.22-7.19(\mathrm{~m}, 3 \mathrm{H}), 4.32(\mathrm{t}, J=7.0 \mathrm{~Hz}, 1 \mathrm{H}), 2.99(\mathrm{~s}, 3 \mathrm{H}), 2.98$ $(\mathrm{s}, 3 \mathrm{H}), 2.78(\mathrm{t}, J=7.3 \mathrm{~Hz}, 2 \mathrm{H}), 2.38-2.26(\mathrm{~m}, 2 \mathrm{H}) ;{ }^{13} \mathrm{C}$ NMR (151 MHz, CDCl 3$): \delta=168.5,140.5,128.7(2 \mathrm{C})$, 128.7 (2C) , 126.4 , 53.0 , 37.3 , 36.3 , 35.9 , 32.3 , 32.3; HRMS (ESI): calculated for $[\mathrm{M}+\mathrm{Na}]^{+}\left(\mathrm{C}_{12} \mathrm{H}_{16} \mathrm{BrNONa}\right) \mathrm{m} / \mathrm{z}$ : 292.0313 found [M+Na] $]^{+}:$292.0303; IR (neat, $\mathrm{cm}^{-1}$ ): 3026, 2929, 2859, 1652, 1494, 1453, 1400, 1135. 
1bc: 2-lodo-N,N-dimethyl-4-phenylbutanamide<smiles>CN(C)C(=O)C(I)CCc1ccccc1</smiles>

The product was prepared according to general procedure D. Purification by flash column chromatography (EtOAc: heptane $=1: 4-2: 1$ ) yielded the product as a yellow oil $(57.0 \mathrm{mg}, 90 \%)$. (Rotamers present in a 1:3 ratio at room temperature)

${ }^{1} \mathrm{H}$ NMR $\left(600 \mathrm{MHz}, \mathrm{CDCl}_{3}\right): \delta=7.30-7.28(\mathrm{~m}, 2 \mathrm{H}), 7.22-7.16(\mathrm{~m}, 3 \mathrm{H}), 4.32(\mathrm{t}, J=7.3 \mathrm{~Hz}, 1 \mathrm{H}), 2.99-2.97(\mathrm{~m}$, $1 \mathrm{H}), 2.97(\mathrm{~s}, 3 \mathrm{H}), 2.87(\mathrm{~s}, 3 \mathrm{H}), 2.70-2.65(\mathrm{~m}, 2 \mathrm{H}), 2.45-2.30(\mathrm{~m}, 2 \mathrm{H}) ;{ }^{13} \mathrm{C} \mathbf{N M R}\left(151 \mathrm{MHz}, \mathrm{CDCl}_{3}\right): \delta=170.1$, 140.3 , 128.7 (2C) , 128.7 (2C) , 128.6 , 126.4 , 53.0 (minor) , 38.1 (major) , 37.8 (major), 37.3 (minor), 36.6 (major) , 36.3 (minor), 35.9 (minor) , 35.2 (major) , 32.4 (minor) , 19.9 (major); HRMS (ESI): calculated for [M+Na] ${ }^{+}$ $\left(\mathrm{C}_{12} \mathrm{H}_{16} \mathrm{INONa}_{\mathrm{N}} \mathrm{m} / \mathrm{z}: 340.0174\right.$ found [M+Na] ${ }^{+}$: 340.0170; IR (neat, cm $^{-1}$ ): 2927, 2857, 1645, 1494, 1453, 1399.

1ca: 2,6-Dichloro-1-(pyrrolidin-1-yl)hexan-1-one<smiles>O=C(C(Cl)CCCCCl)N1CCCC1</smiles>

The product was prepared according to general procedure D. Purification by flash column chromatography (EtOAc: heptane $=1: 4-3: 2$ ) yielded the product as a yellow oil ( $44.6 \mathrm{mg}, 94 \%$ ).

${ }^{1} \mathrm{H}$ NMR $\left(600 \mathrm{MHz}, \mathrm{CDCl}_{3}\right): \delta=4.29-4.26(\mathrm{~m}, 1 \mathrm{H}), 3.69-3.67(\mathrm{~m}, 1 \mathrm{H}), 3.54-3.44(\mathrm{~m}, 5 \mathrm{H}), 2.12-2.06(\mathrm{~m}, 1 \mathrm{H})$, $2.03-1.93(\mathrm{~m}, 3 \mathrm{H}), 1.91-1.88(\mathrm{~m}, 2 \mathrm{H}), 1.83-1.81(\mathrm{~m}, 2 \mathrm{H}), 1.66-1.64(\mathrm{~m}, 1 \mathrm{H}), 1.51-1.49(\mathrm{~m}, 1 \mathrm{H}) ;{ }^{13} \mathrm{C}$ NMR (151 MHz, $\mathrm{CDCl}_{3}$ ): $\delta=166.9,55.8,46.6,46.5,44.8,33.5,32.2$, 26.2 , 24.3 , 23.9; HRMS (ESI): calculated for $[\mathrm{M}+\mathrm{Na}]^{+}\left(\mathrm{C}_{10} \mathrm{H}_{17} \mathrm{Cl}_{2} \mathrm{NONa}\right) \mathrm{m} / \mathrm{z}: 260.0585$ found $[\mathrm{M}+\mathrm{Na}]^{+}:$260.0570; IR (neat, $\mathbf{c m}^{-1}$ ): 2954, 2875, 1650, 1440, 1314.

\section{1cb: 2-Bromo-6-chloro-1-(pyrrolidin-1-yl)hexan-1-one}<smiles>O=C(C(Br)CCCCCl)N1CCCC1</smiles>

The product was prepared according to general procedure D. Purification by flash column chromatography (EtOAc: heptane = 1:4-3:2) yielded the product as a yellow oil (44.5 mg, 79\%). 
${ }^{1} \mathrm{H}$ NMR $\left(600 \mathrm{MHz}, \mathrm{CDCl}_{3}\right): \delta=4.29-4.27(\mathrm{~m}, 1 \mathrm{H}), 3.71-3.67(\mathrm{~m}, 1 \mathrm{H}), 3.57-3.43(\mathrm{~m}, 5 \mathrm{H}), 2.12-2.06(\mathrm{~m}, 1 \mathrm{H})$, $2.01-1.98(\mathrm{~m}, 3 \mathrm{H}), 1.91-1.87(\mathrm{~m}, 2 \mathrm{H}), 1.83-1.80(\mathrm{~m}, 2 \mathrm{H}), 1.68-1.60(\mathrm{~m}, 1 \mathrm{H}), 1.54-1.47(\mathrm{~m}, 1 \mathrm{H}) ;{ }^{13} \mathrm{C}$ NMR (151 MHz, $\mathrm{CDCl}_{3}$ ): $\delta=166.9,55.8,46.6,46.5,44.8,33.6,32.2,26.2,24.3,23.9 ;$ HRMS (ESI): calculated for $[\mathrm{M}+\mathrm{H}]^{+}\left(\mathrm{C}_{10} \mathrm{H}_{17} \mathrm{BrClNO}\right) \mathrm{m} / \mathrm{z}: 282.0260$ found $[\mathrm{M}+\mathrm{H}]^{+}:$282.0250; IR (neat, $\left.\mathbf{c m}^{-1}\right):$ 2954, 2874, 1674, 1438, 1340, $1311,736,648$.

1cc: 2-lodo-6-chloro-1-(pyrrolidin-1-yl)hexan-1-one<smiles>O=C(C(I)CCCCCl)N1CCCC1</smiles>

The product was prepared according to general procedure D. Purification by flash column chromatography (EtOAc: heptane $=1: 4-3: 2)$ yielded the product as a yellow oil $(59.8 \mathrm{mg}, 91 \%$ ). (Rotamers present with a ratio of $6 / 4$ at room temperature)

${ }^{1} \mathrm{H}$ NMR $\left(700 \mathrm{MHz}, \mathrm{CDCl}_{3}\right): \delta=4.27(\mathrm{t}, J=7.4 \mathrm{~Hz}, 1 \mathrm{H}), 3.69-3.66\{\operatorname{minor}\}(\mathrm{m}, 0.4 \mathrm{H}), 3.57-3.50(\mathrm{~m}, 3 \mathrm{H}), 3.49-$ $3.42(\mathrm{~m}, 2 \mathrm{H}), 3.33-3.29$ \{major\} $(\mathrm{m}, 0.6 \mathrm{H}), 2.10-2.03(\mathrm{~m}, 2 \mathrm{H}), 2.02-1.94(\mathrm{~m}, 2 \mathrm{H}), 1.91-1.79(\mathrm{~m}, 4 \mathrm{H}), 1.67-$ $1.56(\mathrm{~m}, 1 \mathrm{H}), 1.51-1.40(\mathrm{~m}, 1 \mathrm{H}) ;{ }^{13} \mathrm{C}$ NMR (176 MHz, $\left.\mathrm{CDCl}_{3}\right): \delta=168.5$ (major), 166.8 (minor) , 55.7 (minor) , 47.0 , 46.8 , 44.8 , 35.6 , 32.0 , 27.1, 26.2 , $24.4,22.9$ (major); HRMS (ESI): calculated for [M+Na] ${ }^{+}\left(\mathrm{C}_{10} \mathrm{H}_{17} \mathrm{ClINONa)}\right.$ m/z: 351.9941 found [M+Na] $]^{+}$351.9936; IR (neat, $\mathbf{c m}^{-1}$ ): 2951, 2872, 1642, 1431, 1341.

1da: Methyl 8-chloro-9-oxo-9-(pyrrolidin-1-yl)nonanoate<smiles>COC(=O)CCCCCCC(Cl)C(=O)N1CCCC1</smiles>

The product was prepared according to general procedure D. Purification by flash column chromatography (EtOAc: heptane = 1:4-1:1) yielded the product as a yellow oil (49.7 mg, 86\%).(Rotamers present)

${ }^{1} \mathrm{H}$ NMR $\left(600 \mathrm{MHz}, \mathrm{CDCl}_{3}\right): \delta=4.28-4.25(\mathrm{~m}, 1 \mathrm{H}), 3.66(\mathrm{~s}, 3 \mathrm{H}), 3.54-3.48(\mathrm{~m}, 2 \mathrm{H}), 3.47-3.42(\mathrm{~m}, 1 \mathrm{H}), 2.30(\mathrm{t}$, $J=7.5 \mathrm{~Hz}, 2 \mathrm{H}), 2.04-1.97(\mathrm{~m}, 3 \mathrm{H}), 1.94-1.86(\mathrm{~m}, 3 \mathrm{H}), 1.64-1.60(\mathrm{~m}, 2 \mathrm{H}), 1.48-1.44(\mathrm{~m}, 1 \mathrm{H}), 1.37-1.32(\mathrm{~m}$, 6H); ${ }^{13} \mathrm{C} \mathrm{NMR} \mathrm{(151} \mathrm{MHz,} \mathrm{CDCl}_{3}$ ): $\delta=174.4,167.2$, 56.1 , 51.7 , 46.6 , 46.5 , 34.2 , 34.1 , 29.0 , 28.9 , 26.3 , 26.2 , 24.9 , 24.3; HRMS (ESI): calculated for $[\mathrm{M}+\mathrm{Na}]^{+}\left(\mathrm{C}_{14} \mathrm{H}_{24} \mathrm{ClNO}_{3} \mathrm{Na}\right) \mathrm{m} / \mathrm{z}: 312.1337$ found $[\mathrm{M}+\mathrm{Na}]^{+}: 312.1338$; IR (neat, $\mathbf{c m}^{-1}$ ): 2927, 2856, 1735, 1651, 1436, 1341, 1170. 
1db: Methyl 8-bromo-9-oxo-9-(pyrrolidin-1-yl)nonanoate<smiles>COC(=O)CCCCCCC(Br)C(=O)N1CCCC1</smiles>

The product was prepared according to general procedure D. Purification by flash column chromatography (EtOAc: heptane $=1: 4-1: 1$ ) yielded the product as a yellow oil $(59.9 \mathrm{mg}, 90 \%)$. (Rotamers present with a ratio of $1: 3$ at room temperature using ${ }^{13} \mathrm{CNMR}$ )

${ }^{1} \mathrm{H}$ NMR $\left(600 \mathrm{MHz}, \mathrm{CDCl}_{3}\right): \delta=4.27(\mathrm{t}, J=7.2 \mathrm{~Hz}, 1 \mathrm{H}), 3.68(\mathrm{~m}, 3 \mathrm{H}), 3.55-3.36(\mathrm{~m}, 3 \mathrm{H}), 2.30(\mathrm{t}, J=7.5 \mathrm{~Hz}, 2 \mathrm{H})$, $2.01-1.98(\mathrm{~m}, 2 \mathrm{H}), 1.91-1.87(\mathrm{~m}, 3 \mathrm{H}), 1.65-1.60(\mathrm{~m}, 2 \mathrm{H}), 1.46-1.44(\mathrm{~m}, 1 \mathrm{H}), 1.36-1.33(\mathrm{~m}, 6 \mathrm{H}) ;{ }^{13} \mathrm{C}$ NMR (151 MHz, $\mathrm{CDCl}_{3}$ ): $\delta=174.4,167.3$ (minor), 167.2 (major) , 56.1 , 51.7 , 46.7 , 46.6 , 46.5, 46.5 , 46.1 , 34.6 , 34.2, $34.1,29.0,28.9,28.9,27.5,26.3,26.2,26.2,24.9,24.4,24.3$; HRMS (ESI): calculated for [M+Na] ${ }^{+}$ $\left(\mathrm{C}_{14} \mathrm{H}_{24} \mathrm{BrNO}_{3} \mathrm{Na}\right) \mathrm{m} / \mathrm{z}: 356.0832$ found $[\mathrm{M}+\mathrm{Na}]^{+}:$356.0832; IR (neat, $\left.\mathrm{cm}^{-1}\right)$ : 2930, 2858, 1734, 1649, 1434, 1340, 1193, 1169.

1dc: Methyl 8-iodo-9-oxo-9-(pyrrolidin-1-yl)nonanoate<smiles>COC(=O)CCCCCCC(I)C(=O)N1CCCC1</smiles>

The product was prepared according to general procedure D. Purification by flash column chromatography (EtOAc: heptane $=1: 4-3: 2)$ yielded the product as a yellow oil $(64.8 \mathrm{mg}, 85 \%)$. (Rotamers present with a ratio of $1 / 2$ at room temperature using ${ }^{13} \mathrm{CNMR}$ )

${ }^{1} \mathrm{H}$ NMR $\left(600 \mathrm{MHz}, \mathrm{CDCl}_{3}\right): \delta=4.27(\mathrm{t}, J=7.3 \mathrm{~Hz}, 1 \mathrm{H}), 3.66(\mathrm{~s}, 3 \mathrm{H}), 3.51-3.48(\mathrm{~m}, 1 \mathrm{H}), 3.45-3.43(\mathrm{~m}, 2 \mathrm{H}), 3.32$ - $3.28(\mathrm{~m}, 1 \mathrm{H}), 2.30-2.27(\mathrm{~m}, 2 \mathrm{H}), 2.03-1.99(\mathrm{~m}, 4 \mathrm{H}), 1.90-1.85(\mathrm{~m}, 2 \mathrm{H}), 1.61-1.59(\mathrm{~m}, 2 \mathrm{H}), 1.35-1.25(\mathrm{~m}$, 6H); ${ }^{13} \mathrm{C}$ NMR (151 MHz, CDCl 3 ): $\delta=174.4,168.8$ (major), 167.2 (minor) , 56.1 (minor) , 51.7 , 46.9 (2C, major) , 46.7 (major) , 46.6 (minor) , 46.5 (minor), 36.2 , 34.2 , 34.1 , 29.5 , 29.0 , 29.0 , 28.9 , $28.7,26.2$, 26.2 , 24.9 , 24.4 , 24.3 , 23.7 (major); HRMS (ESI): calculated for $[\mathrm{M}+\mathrm{Na}]^{+}\left(\mathrm{C}_{14} \mathrm{H}_{24} \mathrm{INO}_{3} \mathrm{Na}\right) \mathrm{m} / \mathrm{z}: 404.0693$ found [M+Na]+: 404.0693 ; IR (neat, cm ${ }^{-1}$ ): 2924, 2854, 1734, 1642, 1431, 1340, 1249. 
1e: 2-lodo-1-(pyrrolidin-1-yl)undecane-1,10-dione<smiles>CC(=O)CCCCCCCC(I)C(=O)N1CCCC1</smiles>

The product was prepared according to general procedure D. Purification by flash column chromatography (EtOAc: heptane $=1: 4-1: 1$ ) yielded the product as a yellow oil $(58.3 \mathrm{mg}, 77 \%)$. (Rotamers present with a ratio of 1:1 at room temperature using $\left.{ }^{13} \mathrm{C} N \mathrm{MR}\right)$

${ }^{1} \mathrm{H}$ NMR $\left(600 \mathrm{MHz}, \mathrm{CDCl}_{3}\right): \delta=4.27(\mathrm{t}, J=7.3 \mathrm{~Hz}, 1 \mathrm{H}), 3.68-3.66(\mathrm{~m}, 0.7 \mathrm{H}$, major), $3.53-3.42(\mathrm{~m}, 3 \mathrm{H}), 3.32-$ $3.30(\mathrm{~m}, 0.4 \mathrm{H}$, minor), $2.41(\mathrm{t}, J=7.4 \mathrm{~Hz}, 2 \mathrm{H}), 2.13(\mathrm{~s}, 3 \mathrm{H}), 2.03-2.00(\mathrm{~m}, 3 \mathrm{H}), 1.92-1.84(\mathrm{~m}, 2 \mathrm{H}), 1.56-1.54$ (m, 2H), 1-45 - $1.43(\mathrm{~m}, 1 \mathrm{H}), 1.35-1.19(\mathrm{~m}, 8 \mathrm{H}) ;{ }^{13} \mathrm{C} \mathrm{NMR} \mathrm{(151} \mathrm{MHz,} \mathrm{CDCl} 3$ ): $\delta=209.5,168.8$ (major), 167.2 (minor) , 56.2 , 46.9, $46.7,46.6,46.5,43.9$, 36.3 , 34.3 , 30.1 , 29.3 , 29.1(2C) , $26.4,26.2$, 26.2 , 24.4 , 24.3 , 23.9; HRMS (ESI): calculated for $[\mathrm{M}+\mathrm{Na}]^{+}\left(\mathrm{C}_{15} \mathrm{H}_{26} \mathrm{INONa}\right) \mathrm{m} / \mathrm{z}: 402.0906$ found $[\mathrm{M}+\mathrm{Na}]^{+}: 402.0897$; IR (neat, $\mathrm{cm}^{-}$ $\left.{ }^{1}\right):$ 2926, 2855, 1721, 1648, 1433, 1358, 1167.

\section{1f: 6-lodo-7-oxo-7-(pyrrolidin-1-yl)heptanenitrile}<smiles>N#CCCCCC(I)C(=O)N1CCCC1</smiles>

The product was prepared according to general procedure D. Purification by flash column chromatography (EtOAc: heptane $=1: 1-20: 1$ ) yielded the product as a yellow oil $(57.6 \mathrm{mg}, 90 \%)$. (Rotamers present with a ratio of $4 / 1$ at room temperature using ${ }^{13} \mathrm{C} N \mathrm{NM}$ )

${ }^{1} \mathrm{H}$ NMR $\left(600 \mathrm{MHz}, \mathrm{CDCl}_{3}\right): \delta=4.27-4.25(\mathrm{~m}, 1 \mathrm{H}), 3.70-3.66(\mathrm{~m}, 0.2 \mathrm{H}$, minor) $, 3.51-3.43(\mathrm{~m}, 3 \mathrm{H}), 3.32-3.28$ (m, 0.8H, major), $2.39-2.34(\mathrm{~m}, 2 \mathrm{H}), 2.10-1.99(\mathrm{~m}, 4 \mathrm{H}), 1.91-1.85(\mathrm{~m}, 2 \mathrm{H}), 1.72-1.67(\mathrm{~m}, 2 \mathrm{H}), 1.58-1.53$ (m, 1H), $1.47-1.44(\mathrm{~m}, 1 \mathrm{H}) ;{ }^{13} \mathrm{C}$ NMR (151 MHz, CDCl 3 ): $\delta=168.4$ (major) , 167.7 (minor), 119.6, 55.4 (minor) , 47.0 (major) , 46.8 (major) , 46.6 (minor), 46.5 (minor), 35.5 (major) , 33.4 (minor) , 28.8 , 26.2 , 26.2 , 24.8 , 24.4

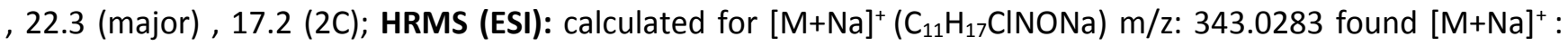
343.0271; IR (neat, $\mathrm{cm}^{-1}$ ): 3362, 2955, 2923, 2853, 2334, 1640, 1432, 1342, 748. 
1g: 2-Bromo-1-(pyrrolidin-1-yl)undec-10-en-1-one<smiles>C=CCCCCCCCC(Br)C(=O)N1CCCC1</smiles>

The product was prepared according to general procedure D. Purification by flash column chromatography (EtOAc: heptane $=1: 3-2: 1$ ) yielded the product as a yellow oil $(53.5 \mathrm{mg}, 85 \%)$.

${ }^{1} \mathrm{H}$ NMR $\left(600 \mathrm{MHz}, \mathrm{CDCl}_{3}\right): \delta=5.82-5.76(\mathrm{~m}, 1 \mathrm{H}), 4.99-4.91(\mathrm{~m}, 2 \mathrm{H}), 4.28-4.26(\mathrm{~m}, 1 \mathrm{H}), 3.68-3.65(\mathrm{~m}, 1 \mathrm{H})$, $3.51-3.42(\mathrm{~m}, 3 \mathrm{H}), 2.04-1.96(\mathrm{~m}, 5 \mathrm{H}), 1.93-1.86(\mathrm{~m}, 3 \mathrm{H}), 1.37-1.29(\mathrm{~m}, 10 \mathrm{H}) ;{ }^{13} \mathrm{C} \mathrm{NMR}\left(151 \mathrm{MHz}, \mathrm{CDCl}_{3}\right): \delta$ $=167.2$, $139.3,114.3,56.2,46.6,46.5,34.3$, 33.9 , $29.4,29.2$, 29.1 , 29.0 , 26.5 , 26.2 , 24.3; HRMS (ESI): calculated for $[\mathrm{M}+\mathrm{Na}]^{+}\left(\mathrm{C}_{15} \mathrm{H}_{26} \mathrm{BrNONa}\right) \mathrm{m} / \mathrm{z}$ : 338.1095 found $[\mathrm{M}+\mathrm{Na}]^{+}:$338.1073; IR (neat, $\left.\mathbf{c m}^{-1}\right): 2924,2853$, $1738,1651,1435,909$.

\section{1h: 2-Chloro-1-(pyrrolidin-1-yl)hex-5-yn-1-one}<smiles>C#CCCC(Cl)C(=O)N1CCCC1</smiles>

The product was prepared according to general procedure D. Purification by flash column chromatography (EtOAc: heptane $=1: 4-2: 1$ ) yielded the product as a yellow oil $(29.8 \mathrm{mg}, 75 \%)$. (Rotamers present with a ration of $1: 10$ in ${ }^{13}$ CNMR)

${ }^{1} \mathrm{H}$ NMR $\left(600 \mathrm{MHz}, \mathrm{CDCl}_{3}\right): \delta=4.59-4.56(\mathrm{~m}, 1 \mathrm{H}), 3.71-3.67(\mathrm{~m}, 1 \mathrm{H}), 3.55-3.47(\mathrm{~m}, 3 \mathrm{H}), 2.47-2.42(\mathrm{~m}, 1 \mathrm{H})$, $2.39-2.35(\mathrm{~m}, 1 \mathrm{H}), 2.28-2.25(\mathrm{~m}, 1 \mathrm{H}), 2.22-2.13(\mathrm{~m}, 1 \mathrm{H}) 2.03-1.98(\mathrm{~m}, 3 \mathrm{H}), 1.94-1.86(\mathrm{~m}, 2 \mathrm{H}) ;{ }^{13} \mathrm{C} \mathrm{NMR}$ (151 MHz, $\mathrm{CDCl}_{3}$ ): $\delta=166.6,82.6,69.8,54.3$, 46.6 (major), 46.5 (major), 45.6 (minor) , 35.7 (minor) , 33.8 (minor) , 32.7 (major) , 26.2 (major) , 24.3 (minor) , 23.0 (major) , 18.0 (minor) , 17.4 (minor) , 15.6 (major); HRMS (ESI): calculated for $[\mathrm{M}+\mathrm{Na}]^{+}\left(\mathrm{C}_{10} \mathrm{H}_{14} \mathrm{ClNONa}\right) \mathrm{m} / \mathrm{z}: 222.0662$ found [M+Na] $]^{+}: 222.0648$; IR (neat, $\left.\mathbf{c m}^{-1}\right): 3296$, $3239,2972,2877,1737,1646,1440,1342$.

\section{1i: 1-(Azepan-1-yl)-2-iodo-4-phenylbutan-1-one}<smiles>O=C(C(I)CCc1ccccc1)N1CCCCCC1</smiles> 
The product was prepared according to general procedure D. Purification by column chromatography on silica gel (10-40\% EtOAc in heptane) yielded the product $(56.6 \mathrm{mg}, 76 \%)$ as a pale yellow oil. (Rotamers present in a

\section{$1: 4$ ratio at room temperature)}

${ }^{1} \mathrm{H}$ NMR $\left(400 \mathrm{MHz}, \mathrm{CDCl}_{3}\right): \delta=7.32-7.26(\mathrm{~m}, 2 \mathrm{H}), 7.23-7.16(\mathrm{~m}, 3 \mathrm{H}), 4.32(\mathrm{apt}, J=7.4 \mathrm{~Hz}, 1 \mathrm{H}), 3.74-3.66$ $\{$ major\} $(\mathrm{m}, 0.8 \mathrm{H}), 3.64-3.56\{\operatorname{minor}\}(\mathrm{m}, 0.2 \mathrm{H}), 3.51-3.17(\mathrm{~m}, 3 \mathrm{H}), 2.80-2.74\{$ minor $\}(\mathrm{m}, 0.4 \mathrm{H}), 2.74-2.62$ $\left\{\right.$ major\}(m, 1.6H), $2.50-2.26(\mathrm{~m}, 2 \mathrm{H}), 1.84-1.36(\mathrm{~m}, 8 \mathrm{H}) ;{ }^{13} \mathrm{C} \mathrm{NMR}\left(100 \mathrm{MHz}, \mathrm{CDCl}_{3}\right): \delta=169.8,140.4,128.7$ (2C), 128.6 (2C), 126.4, 53.1 (minor), 48.4 (major), 47.9 (minor), 46.8 (minor), 46.6 (major), 38.3, 36.1 (minor), 35.2 (major), 32.4 (minor), 29.2 (minor), 28.7 (major), 27.6 (major), 27.5 (minor), 27.2 (major), 27.0 (major), 26.8 (minor), 20.6 (major); HRMS (ESI): calculated for [M+Na] ${ }^{+}\left(\mathrm{C}_{16} \mathrm{H}_{22} \mathrm{INNaO}\right) 394.0638$, found 394.0642; IR (neat, $\mathbf{c m}^{-}$ $\left.{ }^{1}\right)$ : = 2926, 2854, 1639, 1429, 1192, 1152, 749, 700.

\section{2a: 2-(Benzyloxy)-4-phenyl-1-(pyrrolidin-1-yl)butan-1-one}

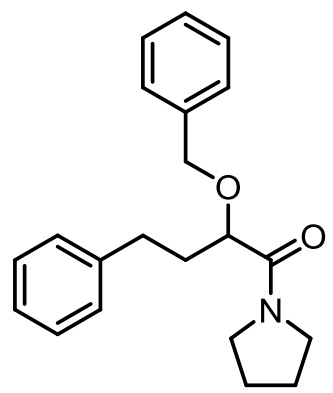

The product was prepared according to general procedure E. Purification by flash column chromatography (EtOAc: heptane $=1: 4-1: 1$ ) yielded the product as a yellow oil $(52.3 \mathrm{mg}, 81 \%)$ (rotamer ratio of 1:4 in ${ }^{1} \mathrm{HNMR}$ ).

${ }^{1} \mathrm{H}$ NMR $\left(600 \mathrm{MHz}, \mathrm{CDCl}_{3}\right): \delta=7.39-7.33(\mathrm{~m}, 4 \mathrm{H}), 7.33-7.28(\mathrm{~m}, 1 \mathrm{H}), 7.26-7.22(\mathrm{~m}, 2 \mathrm{H}), 7.19-7.15(\mathrm{~m}, 1 \mathrm{H})$, $7.15-7.11(\mathrm{~m}, 2 \mathrm{H}), 4.68-4.62(\mathrm{~m}, 1 \mathrm{H}, \mathrm{R} 1 / \mathrm{R} 2), 4.38$ (d, J=11.8 Hz, 0.8H, Rmajor), 4.35 (d, J = $11.8 \mathrm{~Hz}, 0.2 \mathrm{H}$, Rminor), 4.16 (dd, J = 9.1, 4.1 Hz, 0.2H, Rminor), 4.01 (dd, J = 9.1, $4.1 \mathrm{~Hz}, 0.8 \mathrm{H}$, Rmajor), $3.54-3.42(\mathrm{~m}, 2 \mathrm{H}$ ), 3.31 $-3.20(\mathrm{~m}, 2 \mathrm{H}), 2.89-2.84(\mathrm{~m}, 1 \mathrm{H}), 2.73-2.64(\mathrm{~m}, 1 \mathrm{H}), 2.18-2.10(\mathrm{~m}, 1 \mathrm{H}), 1.98-1.92(\mathrm{~m}, 1 \mathrm{H}), 1.86-1.73(\mathrm{~m}$, 4H); ${ }^{13} \mathrm{C}$ NMR (151 MHz, $\left.\mathrm{CDCl}_{3}\right): \delta=170.6,141.6,137.9,128.7,128.6,128.5,128.3,128.0,128.0,126.1$, $126.1,77.9$, 71.5 , $71.5,46.4,46.0,33.8$, 31.9 , 26.5 , 23.8; HRMS (ESI): calculated for $[\mathrm{M}+\mathrm{Na}]^{+}\left(\mathrm{C}_{21} \mathrm{H}_{25} \mathrm{NO}_{2} \mathrm{Na}\right)$ m/z: 346.1783 found [M+Na] $]^{+}$: 346.1777; IR (neat, cm$^{-1}$ ): 3060, 3027, 2950, 2926, 2871, 1641, 1495, 1433. 


\section{2b: 2-(allyloxy)-4-phenyl-1-(pyrrolidin-1-yl)butan-1-one}<smiles>C=CCOC(CCc1ccccc1)C(=O)N1CCCC1</smiles>

The product was prepared according to general procedure E. Purification by flash column chromatography (EtOAc: heptane = 1:4-2:1) yielded the product as a yellow oil $(39.3 \mathrm{mg}, 72 \%$ ).

${ }^{1} \mathrm{H}$ NMR $\left(600 \mathrm{MHz}, \mathrm{CDCl}_{3}\right): \delta=7.34-7.32(\mathrm{~m}, 2 \mathrm{H}), 7.26-7.25(\mathrm{~m}, 3 \mathrm{H}), 6.01-5.97(\mathrm{~m}, 1 \mathrm{H}), 5.35-5.32(\mathrm{~m}, 1 \mathrm{H})$, $5.25-5.23(\mathrm{~m}, 1 \mathrm{H}), 4.16-4.13(\mathrm{~m}, 1 \mathrm{H}), 4.04-4.02(\mathrm{~m}, 1 \mathrm{H}), 3.93-3.90(\mathrm{~m}, 1 \mathrm{H}), 3.56-3.52(\mathrm{~m}, 2 \mathrm{H}), 3.48-3.45$ $(\mathrm{m}, 1 \mathrm{H}), 3.38-3.35(\mathrm{~m}, 1 \mathrm{H}), 2.92-2.88(\mathrm{~m}, 1 \mathrm{H}), 2.80-2.75(\mathrm{~m}, 1 \mathrm{H}), 2.16-2.13(\mathrm{~m}, 1 \mathrm{H}), 2.01-1.97(\mathrm{~m}, 1 \mathrm{H})$, $1.96-1.93(\mathrm{~m}, 2 \mathrm{H}), 1.87-1.83(\mathrm{~m}, 2 \mathrm{H}) ;{ }^{13} \mathrm{C} \mathrm{NMR}\left(151 \mathrm{MHz}, \mathrm{CDCl}_{3}\right): \delta=170.6,141.5,134.6,128.7(2 \mathrm{C}), 128.5$ (2C) $, 126.1,117.6,78.1,70.6,46.4,46.0,33.7,31.8,26.5$, 23.8; HRMS (ESI): calculated for [M+Na] ${ }^{+}$ $\left(\mathrm{C}_{17} \mathrm{H}_{23} \mathrm{NO}_{2} \mathrm{Na}\right) \mathrm{m} / \mathrm{z}: 296.1626$ found $[\mathrm{M}+\mathrm{Na}]^{+}:$296.1622; IR (neat, $\mathrm{cm}^{-1}$ ): 3025, 2952, 2927, 2873, 1637, 1495, 1434.

\section{2c: 2-((3-Methylbut-2-en-1-yl)oxy)-4-phenyl-1-(pyrrolidin-1-yl)butan-1-one}

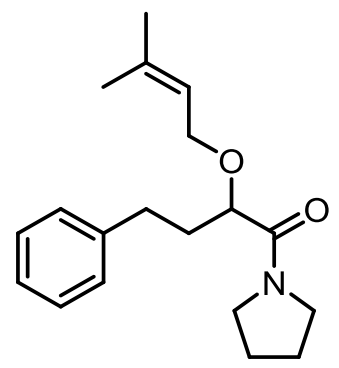

The product was prepared according to general procedure E. Purification by flash column chromatography (EtOAc: heptane $=1: 3-1: 1$ ) yielded the product as a yellow oil $(51.8 \mathrm{mg}, 86 \%)$.

${ }^{1} \mathrm{H}$ NMR $\left(600 \mathrm{MHz}, \mathrm{CDCl}_{3}\right): \delta=7.28-7.26(\mathrm{~m}, 2 \mathrm{H}), 7.20-7.19(\mathrm{~m}, 2 \mathrm{H}), 7.19-7.17(\mathrm{~m}, 1 \mathrm{H}), 5.38-5.35(\mathrm{~m}, 1 \mathrm{H})$, $4.04-4.02(m, 1 H), 3.96-3.94(m, 1 H), 3.90-3.88(m, 1 H), 3.50-3.47(m, 2 H), 3.39-3.37(m, 1 H), 3.34-3.31$ $(\mathrm{m}, 1 \mathrm{H}), 2.85-2.82(\mathrm{~m}, 1 \mathrm{H}), 2.73-2.70(\mathrm{~m}, 1 \mathrm{H}), 2.10-2.06(\mathrm{~m}, 1 \mathrm{H}), 1.94-1.92(\mathrm{~m}, 1 \mathrm{H}), 1.88-1.86(\mathrm{~m}, 2 \mathrm{H})$, $1.85-1.81(\mathrm{~m}, 2 \mathrm{H}), 1.75(\mathrm{~s}, 3 \mathrm{H}), 1.64(\mathrm{~s}, 3 \mathrm{H}) ;{ }^{13} \mathrm{C} \mathrm{NMR}\left(151 \mathrm{MHz}, \mathrm{CDCl}_{3}\right): \delta=170.9,141.6,137.6,128.7$ (2C) , 128.5 (2C) , $126.1,120.9,77.9,65.9,46.4,46.0,33.7,31.9$, 26.6 , 26.0 , 23.8 , 18.2; HRMS (ESI): calculated for $\left(\mathrm{C}_{19} \mathrm{H}_{27} \mathrm{NO}_{2} \mathrm{Na}\right)[\mathrm{M}+\mathrm{Na}]^{+} \mathrm{m} / \mathrm{z}: 324.1939$ found $[\mathrm{M}+\mathrm{Na}]^{+}:$324.1934; IR (neat, $\left.\mathbf{c m}^{-1}\right)$ : 3025, 2952, 2930, 2878, 1639, 1495, 1436. 
2d: 2-(4-Nitrophenoxy)-4-phenyl-1-(pyrrolidin-1-yl)butan-1-one

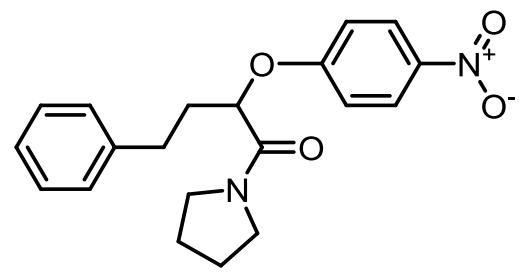

The product was prepared according to general procedure E. Purification by column chromatography on silica gel (EtOAc: heptane $=1: 1)$ yielded the product $(56.0 \mathrm{mg}, 79 \%)$ as a pale yellow liquid.

${ }^{1} \mathrm{H}$ NMR $\left(400 \mathrm{MHz}, \mathrm{CDCl}_{3}\right): \delta=8.16(\mathrm{~d}, J=9.3 \mathrm{~Hz}, 2 \mathrm{H}), 7.29-7.12(\mathrm{~m}, 5 \mathrm{H}), 6.87(\mathrm{~d}, J=9.3 \mathrm{~Hz}, 2 \mathrm{H}), 4.66(\mathrm{dd}, J=$ 9.1, 4.0 Hz, 1H), 3.53-3.37 (m, 3H), 3.25-3.14 (m, 1H), $2.96-2.87(m, 1 H), 2.85-2.74(m, 1 H), 2.38-2.26(m$, 1H), $2.23-2.13(\mathrm{~m}, 1 \mathrm{H}), 1.94-1.68(\mathrm{~m}, 4 \mathrm{H}) ;{ }^{13} \mathrm{C}$ NMR $\left(100 \mathrm{MHz}, \mathrm{CDCl}_{3}\right): \delta=168.2,162.8,142.3,140.5,128.8$ (2C), 128.7 (2C), 126.6, 126.2 (2C), 115.0 (2C), 77.8, 46.8, 46.1, 33.4, 31.8, 26.6, 23.6; HRMS (ESI): calculated for $[\mathrm{M}+\mathrm{Na}]^{+}\left(\mathrm{C}_{20} \mathrm{H}_{22} \mathrm{~N}_{2} \mathrm{O}_{4} \mathrm{Na}\right) \mathrm{m} / \mathrm{z}: 377.1472$, found 377.1475; IR (neat, $\mathrm{cm}^{-1}$ ): 3082, 3027, 2956, 2878, 1652, 1590, $1512,1494,1441,1338,1256,1110,845,751,655$.

\section{2e: Ethyl 2-((1-oxo-4-phenyl-1-(pyrrolidin-1-yl)butan-2-yl)oxy)cyclohex-1-ene-1-carboxylate}<smiles>CCOC(=O)C1=C(OC(CCc2ccccc2)C(=O)N2CCCC2)CCCC1</smiles>

The product was prepared according to general procedure E. Purification by flash column chromatography (EtOAc: heptane $=1: 2-1: 1$ ) yielded the product as a yellow oil $(74.0 \mathrm{mg}, 96 \%)$.

${ }^{1} \mathrm{H}$ NMR $\left(600 \mathrm{MHz}, \mathrm{CDCl}_{3}\right): \delta=7.28-7.26(\mathrm{~m}, 2 \mathrm{H}), 7.20-7.18(\mathrm{~m}, 3 \mathrm{H}), 4.56-4.54(\mathrm{~m}, 1 \mathrm{H}), 4.20-4.17(\mathrm{~m}, \mathrm{~J}=$ $7.2 \mathrm{~Hz}, 2 \mathrm{H}), 3.49-3.45(\mathrm{~m}, 4 \mathrm{H}), 2.88-2.84(\mathrm{~m}, 1 \mathrm{H}), 2.82-2.77(\mathrm{~m}, 1 \mathrm{H}), 2.36-2.28(\mathrm{~m}, 2 \mathrm{H}), 2.20-2.17(\mathrm{~m}, 2 \mathrm{H})$, $2.10-2.04(\mathrm{~m}, 2 \mathrm{H}), 1.89-1.76(\mathrm{~m}, 4 \mathrm{H}), 1.64-1.63(\mathrm{~m}, 2 \mathrm{H}), 1.55-1.54(\mathrm{~m}, 2 \mathrm{H}), 1.30-1.28(\mathrm{~m}, 3 \mathrm{H}) ;{ }^{13} \mathrm{C}$ NMR (151 MHz, CDCl $): \delta=169.7,168.1,160.0,141.1,128.7$ (2C) , 128.6 (2C) , 126.2 , 108.1 , $77.0,60.0,46.7$, 46.1, 33.8 , 31.5 , $26.7,26.6,25.6,23.6,22.7,22.1,14.6$; HRMS (ESI): calculated for $\left[\mathrm{M}+\mathrm{Na}^{+}{ }^{+}\left(\mathrm{C}_{23} \mathrm{H}_{31} \mathrm{NO}_{4} \mathrm{Na}\right) \mathrm{m} / \mathrm{z}\right.$ : 408.2151 found [M+Na] $]^{+}: 408.2146$; IR (neat, $\mathrm{cm}^{-1}$ ): 3026, 2934, 2874, 1710, 1635, 1443. 


\section{2f: Methyl 8-(benzyloxy)-9-oxo-9-(pyrrolidin-1-yl)nonanoate}<smiles>COC(=O)CCCCCCC(OCc1ccccc1)C(=O)N1CCCC1</smiles>

The product was prepared according to general procedure E. Purification by flash column chromatography (EtOAc: heptane $=1: 3-1: 1$ ) yielded the product as a yellow oil $(58.5 \mathrm{mg}, 81 \%)$.

${ }^{1} \mathrm{H}$ NMR $\left(600 \mathrm{MHz}, \mathrm{CDCl}_{3}\right): \delta=7.28-7.25(\mathrm{~m}, 4 \mathrm{H}), 7.22-7.19(\mathrm{~m}, 1 \mathrm{H}), 4.57-4.55(\mathrm{~m}, 1 \mathrm{H}), 4.32-4.30(\mathrm{~m}, 1 \mathrm{H})$, $3.95-3.93(\mathrm{~m}, 1 \mathrm{H}), 3.59(\mathrm{~s}, 3 \mathrm{H}), 3.44-3.42(\mathrm{~m}, 2 \mathrm{H}), 3.34-3.31(\mathrm{~m}, 2 \mathrm{H}), 2.22(\mathrm{t}, J=7.8 \mathrm{~Hz}, 2 \mathrm{H}), 1.82-1.80(\mathrm{~m}$, 2H), $1.75-1.69(\mathrm{~m}, 3 \mathrm{H}), 1.47-1.42(\mathrm{~m}, 1 \mathrm{H}), 1.54-1.51(\mathrm{~m}, 1 \mathrm{H}), 1.47-1.42(\mathrm{~m}, 1 \mathrm{H}), 1.28-1.18(\mathrm{~m}, 6 \mathrm{H}) ;{ }^{13} \mathrm{C}$ NMR (151 MHz, $\left.\mathrm{CDCl}_{3}\right): \delta=174.4,170.8,137.9,128.7,128.5,128.3,128.1,127.9,79.0,71.4,66.2$, 51.6 , $46.4,46.1,34.2,32.1,29.1,29.1,26.5,25.6,25.0,23.8$; HRMS (ESI): calculated for $[\mathrm{M}+\mathrm{Na}]^{+}\left(\mathrm{C}_{21} \mathrm{H}_{31} \mathrm{NO}_{4} \mathrm{Na}\right)$ $\mathrm{m} / \mathrm{z}: 384.2151$ found $[\mathrm{M}+\mathrm{Na}]^{+}$: 384.2150; IR (neat, $\mathrm{cm}^{-1}$ ): 3029, 2932, 2860, 1736, 1646, 1495, 1435.

\section{2g: 2-(Benzyloxy)-1-(pyrrolidin-1-yl)undecane-1,10-dione}<smiles>CC(=O)CCCCCCCC(OCc1ccccc1)C(=O)N1CCCC1</smiles>

The product was prepared according to general procedure E. Purification by flash column chromatography (EtOAc: heptane $=1: 4-2: 1$ ) yielded the product as a yellow oil $(54.5 \mathrm{mg}, 76 \%)$.

${ }^{1} \mathrm{H}$ NMR $\left(600 \mathrm{MHz}, \mathrm{CDCl}_{3}\right): \delta=7.28-7.25(\mathrm{~m}, 4 \mathrm{H}), 7.22-7.20(\mathrm{~m}, 1 \mathrm{H}), 4.57-4.55(\mathrm{~m}, 1 \mathrm{H}), 4.32-4.30(\mathrm{~m}, 1 \mathrm{H})$, $3.95(\mathrm{~m}, 1 \mathrm{H}), 3.46-3.43(\mathrm{~m}, 2 \mathrm{H}), 3.41-3.32(\mathrm{~m}, 2 \mathrm{H}), 2.35-2.32(\mathrm{t}, J=7.8 \mathrm{~Hz}, 2 \mathrm{H}), 2.06(\mathrm{~s}, 3 \mathrm{H}), 1.82-1.80(\mathrm{~m}$, 2H), $1.75-1.71(\mathrm{~m}, 3 \mathrm{H}), 1.60-1.57(\mathrm{~m}, 1 \mathrm{H}), 1.48-1.43(\mathrm{~m}, 2 \mathrm{H}), 1.26-1.18(\mathrm{~m}, 8 \mathrm{H}) ;{ }^{13} \mathrm{C} \mathrm{NMR}\left(151 \mathrm{MHz}, \mathrm{CDCl}_{3}\right)$ : $\delta=209.6$, 170.8 , 137.9 , $128.5(2 \mathrm{C}), 128.1(2 \mathrm{C}), 127.9$, 79.1 , 71.4 , 46.4 , 46.1 , 43.9 , 32.1 , 30.0 , 29.3 , 29.3 , 29.2 , $26.5,25.7,23.9,23.8$; HRMS (ESI): calculated for $[\mathrm{M}+\mathrm{Na}]^{+}\left(\mathrm{C}_{22} \mathrm{H}_{33} \mathrm{NO}_{3} \mathrm{Na}\right) \mathrm{m} / \mathrm{z}: 382.2358$ found $[\mathrm{M}+\mathrm{Na}]^{+}$:

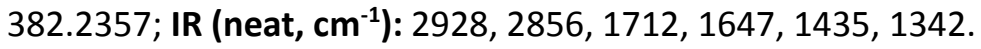




\section{2h: 6-(Benzyloxy)-7-oxo-7-(pyrrolidin-1-yl)heptanenitrile}<smiles>N#CCCCCC(OCc1ccccc1)C(=O)N1CCCC1</smiles>

The product was prepared according to general procedure E. Purification by flash column chromatography (EtOAc: heptane = 1:1-20:1) yielded the product as a yellow oil $(52.2 \mathrm{mg}, 87 \%)$.

${ }^{1} \mathrm{H}$ NMR $\left(600 \mathrm{MHz}, \mathrm{CDCl}_{3}\right): \delta=7.39-7.26(\mathrm{~m}, 5 \mathrm{H}), 4.64(\mathrm{~d}, J=11.8 \mathrm{~Hz}, 1 \mathrm{H}), 4.39(\mathrm{~d}, J=11.8 \mathrm{~Hz}, 1 \mathrm{H}), 4.03(\mathrm{dd}, J=$ 8.5, $4.3 \mathrm{~Hz}, 1 \mathrm{H}), 3.57-3.43(\mathrm{~m}, 3 \mathrm{H}), 3.41(\mathrm{t}, J=6.7 \mathrm{~Hz}, 1 \mathrm{H}), 2.33(\mathrm{t}, J=6.7 \mathrm{~Hz}, 2 \mathrm{H}), 1.94-1.86(\mathrm{~m}, 2 \mathrm{H}), 1.86-$ $1.78(\mathrm{~m}, 3 \mathrm{H}), 1.77-1.59(\mathrm{~m}, 5 \mathrm{H}) .{ }^{13} \mathrm{C}$ NMR $\left(151 \mathrm{MHz}, \mathrm{CDCl}_{3}\right): \delta=170.2,137.7,128.6(2 \mathrm{C}), 128.2(2 \mathrm{C}), 128.1$, $119.7,78.5,71.5,46.5,46.2,31.2,26.6,25.3,25.0,23.8,17.2$; HRMS (ESI): calculated for [M+Na] ${ }^{+}$ $\left(\mathrm{C}_{18} \mathrm{H}_{24} \mathrm{~N}_{2} \mathrm{O}_{2} \mathrm{Na}\right.$ ) m/z: 323.1735 found [M+Na] ${ }^{+}: 323.1722$; IR (neat, $\left.\mathrm{cm}^{-1}\right)$ : 3023, 3010, 2933, 2845, 1640, 1491, 1430.

\section{2i: 2-(Benzyloxy)-6-chloro-1-(pyrrolidin-1-yl)hexan-1-one}<smiles>O=C(C(CCCCCl)OCc1ccccc1)N1CCCC1</smiles>

The product was prepared according to general procedure E. Purification by flash column chromatography (EtOAc: heptane $=1: 4-1: 1$ ) yielded the product as a yellow oil $(51.3 \mathrm{mg}, 83 \%)$.

${ }^{1} \mathrm{H}$ NMR $\left(600 \mathrm{MHz}, \mathrm{CDCl}_{3}\right): \delta=7.27-7.26(\mathrm{~m}, 4 \mathrm{H}), 7.23-7.19(\mathrm{~m}, 1 \mathrm{H}), 4.57-4.55(\mathrm{~m}, 1 \mathrm{H}), 4.32-4.30(\mathrm{~m}, 1 \mathrm{H})$, $3.96-3.95(m, 1 H), 3.47-3.40(m, 4 H), 3.34-3.32(m, 2 H), 1.81-1.77(m, 2 H), 1.76-1.72(m, 3 H), 1.71-1.67$ $(\mathrm{m}, 2 \mathrm{H}), 1.63-1.61(\mathrm{~m}, 2 \mathrm{H}), 1.45-1.42(\mathrm{~m}, 1 \mathrm{H}) ;{ }^{13} \mathrm{C}$ NMR (151 MHz, CDCl 3 ): $\delta=170.5,137.8,128.5$ (2C) , 128.1 (2C) , 128.0 , $78.7,71.4,46.4,46.2$, $44.9,32.4,31.3$, 26.5 , 23.8 , 23.2; HRMS (ESI): calculated for [M+Na] ${ }^{+}$ $\left(\mathrm{C}_{17} \mathrm{H}_{24} \mathrm{ClNO}_{2} \mathrm{Na} \text { ) m/z: } 332.1393 \text { found [M+Na] }\right]^{+}$: 332.1388; IR (neat, $\left.\mathrm{cm}^{-1}\right):$ 2942, 2888, 1640, 1494, 1450. 


\section{2j: 2-(Benzyloxy)-1-(pyrrolidin-1-yl)undec-10-en-1-one}<smiles>C=CCCCCCCCC(OCc1ccccc1)C(=O)N1CCCC1</smiles>

The product was prepared according to general procedure E. Purification by flash column chromatography (EtOAc: heptane $=1: 4-2: 1$ ) yielded the product as a yellow oil $(56.9 \mathrm{mg}, 83 \%)$.

${ }^{1} \mathrm{H}$ NMR $\left(600 \mathrm{MHz}, \mathrm{CDCl}_{3}\right): \delta=7.28-7.22(\mathrm{~m}, 4 \mathrm{H}), 7.21-7.19(\mathrm{~m}, 1 \mathrm{H}), 5.76-5.70(\mathrm{~m}, 1 \mathrm{H}), 4.93-4.85(\mathrm{~m}, 2 \mathrm{H})$, $4.57-4.55(\mathrm{~m}, 1 \mathrm{H}), 4.33-4.31(\mathrm{~m}, 1 \mathrm{H}), 3.96-3.94(\mathrm{~m}, 1 \mathrm{H}), 3.47-3.41(\mathrm{~m}, 2 \mathrm{H}), 3.35-3.32(\mathrm{~m}, 2 \mathrm{H}), 1.97-1.94$ $(\mathrm{m}, 2 \mathrm{H}), 1.82-1.80(\mathrm{~m}, 2 \mathrm{H}), 1.75-1.71(\mathrm{~m}, 2 \mathrm{H}), 1.61-1.58(\mathrm{~m}, 1 \mathrm{H}), 1.44-1.43(\mathrm{~m}, 1 \mathrm{H}), 1.29-1.28(\mathrm{~m}, 3 \mathrm{H})$, $1.20-1.18(\mathrm{~m}, 7 \mathrm{H}) ;{ }^{13} \mathrm{C}$ NMR (151 MHz, $\left.\mathrm{CDCl}_{3}\right): \delta=170.9,139.3,138.0,128.5(2 \mathrm{C}), 128.1(2 \mathrm{C}), 127.9,114.3$, 79.2 , $71.4,46.4,46.1$, 33.9 , 32.2 , 29.5 , 29.5 , 29.2 , 29.0 , 26.6 , 25.8 , 23.8; HRMS (ESI): calculated for [M+Na] ${ }^{+}$ $\left(\mathrm{C}_{22} \mathrm{H}_{33} \mathrm{NO}_{2} \mathrm{Na}\right) \mathrm{m} / \mathrm{z}: 366.2409$ found $[\mathrm{M}+\mathrm{Na}]^{+}:$366.2404; IR (neat, $\mathbf{c m}^{-1}$ ): 3072, 3030, 2971, 2925, 2855, 1639, 1434.

\section{2k: 2-(benzyloxy)-1-(pyrrolidin-1-yl)hex-5-yn-1-one}<smiles>C#CCCC(OCc1ccccc1)C(=O)N1CCCC1</smiles>

The product was prepared according to general procedure E. Purification by flash column chromatography (EtOAc: heptane $=1: 3-2: 1$ ) yielded the product as a yellow oil $(40.0 \mathrm{mg}, 73 \%)$.

${ }^{1} \mathrm{H}$ NMR (600 MHz, $\left.\mathrm{CDCl}_{3}\right)$ ): $7.38-7.32(\mathrm{~m}, 4 \mathrm{H}), 7.32-7.27(\mathrm{~m}, 1 \mathrm{H}), 4.65(\mathrm{~d}, J=11.7 \mathrm{~Hz}, 1 \mathrm{H}), 4.42(\mathrm{~d}, J=11.7$ $\mathrm{Hz}, 1 \mathrm{H}), 4.24(\mathrm{dd}, J=9.2,4.1 \mathrm{~Hz}, 1 \mathrm{H}), 3.59-3.27(\mathrm{~m}, 4 \mathrm{H}), 2.51-2.25(\mathrm{~m}, 2 \mathrm{H}), 2.05-1.96(\mathrm{~m}, 1 \mathrm{H}), 1.94-1.77(\mathrm{~m}$, 6H); $\left.{ }^{13} \mathrm{C} \mathrm{NMR} \mathrm{(151} \mathrm{MHz,} \mathrm{CDCl}\right)$ ) $8: 170.1,137.8,128.5$ (2C) , 128.2 (2C) , $128.0,76.6,71.6,69.1,46.3$, 46.1 , 31.0 , 26.5 , 23.9 , 15.0; HRMS: calculated for $\mathrm{C}_{17} \mathrm{H}_{21} \mathrm{NO}_{2}[\mathrm{M}+\mathrm{Na}]^{+} \mathrm{m} / \mathrm{z}: 294.1470$ found $[\mathrm{M}+\mathrm{Na}]^{+}: 294.1459$; IR (neat, $\mathrm{cm}^{-1}$ ): 3061, 3028, 2938, 2871, 1632, 1492, 1429. 


\section{1: 2-(Benzyloxy)-N,N-dimethyl-4-phenylbutanamide}

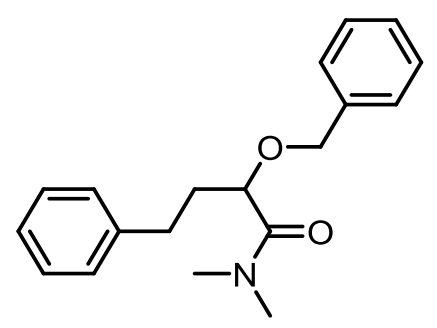

The product was prepared according to general procedure E. Purification by column chromatography on silica gel (EtOAc: heptane $=1: 3)$ yielded the product $(44.0 \mathrm{mg}, 74 \%)$ as a pale yellow liquid.

${ }^{1} \mathrm{H}$ NMR $\left(400 \mathrm{MHz}, \mathrm{CDCl}_{3}\right): \delta=7.40-7.11(\mathrm{~m}, 10 \mathrm{H}), 4.63(\mathrm{~d}, J=11.6 \mathrm{~Hz}, 1 \mathrm{H}), 4.35(\mathrm{~d}, J=11.6 \mathrm{~Hz}, 1 \mathrm{H}), 4.16(\mathrm{dd}, J$ = 9.1, 4.2 Hz, 1H), $2.94(\mathrm{~s}, 3 \mathrm{H}), 2.92(\mathrm{~s}, 3 \mathrm{H}), 2.89-2.81(\mathrm{~m}, 1 \mathrm{H}), 2.74-2.65(\mathrm{~m}, 1 \mathrm{H}), 2.20-2.09(\mathrm{~m}, 1 \mathrm{H}), 2.02-$ $1.92(\mathrm{~m}, 1 \mathrm{H}) ;{ }^{13} \mathrm{C}$ NMR (100 MHz, $\left.\mathrm{CDCl}_{3}\right): \delta=171.7,141.5,137.9,128.7$ (2C), $128.6(2 \mathrm{C}), 128.5(2 \mathrm{C}), 128.3(2 \mathrm{C})$, 128.0, 126.1, 77.5, 71.5, 36.5 , 36.2 , 33.9 , 31.9; HRMS (ESI): calculated for $[\mathrm{M}+\mathrm{Na}]^{+}\left(\mathrm{C}_{19} \mathrm{H}_{23} \mathrm{NO}_{2} \mathrm{Na}\right) \mathrm{m} / \mathrm{z}: 320.1621$, found 320.1621; IR (neat, $\mathrm{cm}^{-1}$ ): 3060, 3026, 2925, 2861, 1639, 1494, 1452, 1397, 1343, 1257, 1097, 981, 734, 697.

2m: 1-(Azepan-1-yl)-2-(benzyloxy)-4-phenylbutan-1-one<smiles>O=C(CCc1ccccc1)C(OCc1ccccc1)N1CCCCCC1</smiles>

The product was prepared according to general procedure E. Purification by column chromatography on silica gel (10-30\% EtOAc in heptane) yielded the product (32.2 mg, 46\% (54\% brsm)) as a pale yellow oil.

${ }^{1} \mathrm{H}$ NMR $\left(400 \mathrm{MHz}, \mathrm{CDCl}_{3}\right): \delta=7.40-7.28(\mathrm{~m}, 5 \mathrm{H}), 7.25-7.09(\mathrm{~m}, 5 \mathrm{H}), 4.67(\mathrm{~d}, J=11.7 \mathrm{~Hz}, 1 \mathrm{H}), 4.35(\mathrm{~d}, J=11.7$ $\mathrm{Hz}, 1 \mathrm{H}), 4.14(\mathrm{dd}, J=11.7 \mathrm{~Hz}, 1 \mathrm{H}), 3.71-3.61(\mathrm{~m}, 1 \mathrm{H}), 3.40-3.20(\mathrm{~m}, 3 \mathrm{H}), 2.91-2.81(\mathrm{~m}, 1 \mathrm{H}), 2.74-2.63(\mathrm{~m}$, $1 \mathrm{H}), 2.23-2.12(\mathrm{~m}, 1 \mathrm{H}), 2.01-1.87(\mathrm{~m}, 1 \mathrm{H}), 1.79-1.65(\mathrm{~m}, 2 \mathrm{H}), 1.57-1.45(\mathrm{~m}, 6 \mathrm{H}) ;{ }^{13} \mathrm{C} \mathrm{NMR}\left(100 \mathrm{MHz}, \mathrm{CDCl}_{3}\right)$ : $\delta=171.3$, 141.6, 138.1, 128.7 (2C), 128.5 (4C), 128.3 (2C), 127.9, 126.1, 76.7, 71.3, 47.0, 46.8, 34.4, 32.0, 29.5, 27.5 (2C), 26.7; HRMS (ESI): calculated for $[\mathrm{M}+\mathrm{Na}]^{+}\left(\mathrm{C}_{23} \mathrm{H}_{29} \mathrm{NNaO}_{2}\right) 374.2091$, found 374.2093; IR (neat, $\left.\mathrm{cm}^{-1}\right):=$ 2925, 2855, 1649, 1454, 1275, 1099, 746, 699. 


\section{2n: Benzyl 8-(benzyloxy)-9-((4-methoxybenzyl)(methyl)amino)-9-oxononanoate}<smiles>COc1ccc(CN(C)C(=O)C(CCCCCCC(=O)OCc2ccccc2)OCc2ccccc2)cc1</smiles>

The product was prepared according to general procedure E. Purification by column chromatography on silica gel (5-50\% EtOAc in heptane) yielded the product $(34.0 \mathrm{mg}, 33 \%)$ as a pale yellow solid. When the nucleophile was added $20 \mathrm{~s}$ after the addition of 2,6-lutidine $\mathrm{N}$-oxide (instead of $5 \mathrm{~min}$ ) the reaction yielded the product in (42.6 $\mathrm{mg}, 41 \%)$. (Rotamers present in a 1:2 ratio at room temperature)

${ }^{1} \mathrm{H}$ NMR $\left(400 \mathrm{MHz}, \mathrm{CDCl}_{3}\right): \delta=7.40-7.27(\mathrm{~m}, 10 \mathrm{H}), 7.19$ \{major\}$(\mathrm{d}, J=8.6 \mathrm{~Hz}, 1.3 \mathrm{H}), 7.03$ \{minor\}$(\mathrm{d}, J=8.6 \mathrm{~Hz}$, $0.7 \mathrm{H}), 6.89-6.82(\mathrm{~m}, 2 \mathrm{H}), 5.11(\mathrm{~s}, 2 \mathrm{H}), 4.69-4.32(\mathrm{~m}, 4 \mathrm{H}), 4.26-4.16(\mathrm{~m}, 1 \mathrm{H}), 3.80(\mathrm{~s}, 3 \mathrm{H}), 2.96-2.86(\mathrm{~m}, 3 \mathrm{H})$, $2.37-2.28(\mathrm{~m}, 2 \mathrm{H}), 1.86-1.21(\mathrm{~m}, 10 \mathrm{H}) ;{ }^{13} \mathrm{C} \mathrm{NMR}\left(100 \mathrm{MHz}, \mathrm{CDCl}_{3}\right): \delta=173.7,171.8,159.2,137.9,136.3,129.7$ (2C), 129.5, 128.8, 128.7 (2C), 128.5 (2C), 128.5, 128.3 (2C), 128.1 (major), 128.1 (minor), 128.0, 127.9, 114.4 (minor), 114.2 (major), 78.9 (major), 78.7 (minor), 71.5 (major), 71.4 (minor), 66.2, 55.4, 52.0, 51.0, 34.4, 34.2 (major), 33.9 (minor), 32.7 (minor), 32.2 (major), 29.1, 25.7, 25.0; HRMS (ESI): calculated for [M+Na] ${ }^{+}$ $\left(\mathrm{C}_{32} \mathrm{H}_{39} \mathrm{NNaO}_{5}\right)$ 540.2720, found 540.2734; IR (neat, $\left.\mathrm{cm}^{-1}\right):=2933,2857,1733,1650,1511,1455,1246,1174$, $738,698$.

\section{3a: 2-(Benzylthio)-4-phenyl-1-(pyrrolidin-1-yl)butan-1-one}<smiles>O=C(C(CCc1ccccc1)SCc1ccccc1)N1CCCC1</smiles>

The product was prepared according to general procedure E. Purification by flash column chromatography (EtOAc: heptane $=1: 4-1: 1$ ) yielded the product as a yellow oil $(63.7 \mathrm{mg}, 94 \%)$.

${ }^{1} \mathrm{H}$ NMR (600 MHz, MeOD): $\delta=7.31-7.28(\mathrm{~m}, 2 \mathrm{H}), 7.27-7.21(\mathrm{~m}, 4 \mathrm{H}), 7.19(\mathrm{~d}, J=7.2 \mathrm{~Hz}, 2 \mathrm{H}), 7.15(\mathrm{~d}, J=7.8$ $\mathrm{Hz}, 2 \mathrm{H}), 3.75(\mathrm{~s}, 2 \mathrm{H}), 3.45-3.35(\mathrm{~m}, 2 \mathrm{H}), 3.30-3.28(\mathrm{~m}, 1 \mathrm{H}), 3.18-3.16(\mathrm{~m}, 1 \mathrm{H}), 2.86-2.82(\mathrm{~m}, 1 \mathrm{H}), 2.78-2.72$ $(\mathrm{m}, 2 \mathrm{H}), 2.61-2.56(\mathrm{~m}, 1 \mathrm{H}), 2.35-2.29(\mathrm{~m}, 1 \mathrm{H}), 2.09-2.03(\mathrm{~m}, 1 \mathrm{H}), 1.91-1.87(\mathrm{~m}, 1 \mathrm{H}), 1.80-1.74(\mathrm{~m}, 4 \mathrm{H}) ;{ }^{13} \mathrm{C}$ NMR (151 MHz, MeOD): $\delta$ = 171.5 , 142.3 , 139.7, 130.2 (2C) , 129.7 (2C) , 129.6 (2C) , 129.5 (2C) , $128.1,127.2$ 
, $47.2,47.0,44.6,35.5,34.7,34.3,26.7,25.1$; HRMS (ESI): calculated for $[\mathrm{M}+\mathrm{Na}]^{+}\left(\mathrm{C}_{21} \mathrm{H}_{25} \mathrm{NOSNa}\right) \mathrm{m} / \mathrm{z}$ : 362.1555 found $[\mathrm{M}+\mathrm{Na}]^{+}:$362.1549; IR (neat, $\mathrm{cm}^{-1}$ ): 3026, 2949, 2872, 1635, 1494, 1428.

3b: 2-(Allylthio)-4-phenyl-1-(pyrrolidin-1-yl)butan-1-one<smiles>C=CCSC(CCc1ccccc1)C(=O)N1CCCC1</smiles>

The product was prepared according to general procedure E. Purification by flash column chromatography (EtOAc: heptane $=1: 3-1: 1$ ) yielded the product as a yellow oil $(46.2 \mathrm{mg}, 80 \%)$.

${ }^{1} \mathrm{H}$ NMR $\left(600 \mathrm{MHz}, \mathrm{CDCl}_{3}\right): \delta=7.28-7.26(\mathrm{~m}, 2 \mathrm{H}), 7.19-7.15(\mathrm{~m}, 3 \mathrm{H}), 5.80-5.74(\mathrm{~m}, 1 \mathrm{H}), 5.03-5.00(\mathrm{~m}, 2 \mathrm{H})$, $3.52-3.46(\mathrm{~m}, 3 \mathrm{H}), 3.23-3.20(\mathrm{~m}, 2 \mathrm{H}), 3.18-3.16(\mathrm{~m}, 1 \mathrm{H}), 3.10-3.08(\mathrm{~m}, 1 \mathrm{H}), 2.79-2.75(\mathrm{~m}, 1 \mathrm{H}), 2.66-2.61$ $(\mathrm{m}, 1 \mathrm{H}), 2.44-2.38(\mathrm{~m}, 1 \mathrm{H}), 2.05-1.99(\mathrm{~m}, 1 \mathrm{H}), 1.91-1.89(\mathrm{~m}, 2 \mathrm{H}), 1.86-1.83(\mathrm{~m}, 2 \mathrm{H}) ;{ }^{13} \mathrm{C}$ NMR (176 MHz, $\left.\mathrm{CDCl}_{3}\right): \delta=169.1,141.3,134.5,128.7(2 \mathrm{C}), 128.5(2 \mathrm{C}), 126.1,117.4,46.3,46.2,43.5,33.5,33.4,32.7,26.2$ , 24.4; HRMS (ESI): calculated for $[\mathrm{M}+\mathrm{Na}]^{+}\left(\mathrm{C}_{17} \mathrm{H}_{23} \mathrm{NOSNa}\right) \mathrm{m} / \mathrm{z}: 312.1398$ found $[\mathrm{M}+\mathrm{Na}]^{+}$: 312.1396; IR (neat, cm $\left.^{-1}\right)$ : 3060, 3024, 2948, 2871, 1633, 1494, 1423, 1340.

\section{3c: 2-(tert-Butylthio)-4-phenyl-1-(pyrrolidin-1-yl)butan-1-one}<smiles>CC(C)(C)SC(CCc1ccccc1)C(=O)N1CCCC1</smiles>

The product was prepared according to general procedure E. Purification by flash column chromatography (EtOAc: heptane = 1:3-4:1) yielded the product as a yellow solid $(32.7 \mathrm{mg}, 76 \%$ ).

${ }^{1} \mathrm{H}$ NMR $\left(600 \mathrm{MHz}, \mathrm{CDCl}_{3}\right): \delta=7.27-7.25(\mathrm{~m}, 2 \mathrm{H}), 7.18-7.16(\mathrm{~m}, 3 \mathrm{H}), 3.48-3.46(\mathrm{~m}, 2 \mathrm{H}), 3.42-3.36(\mathrm{~m}, 2 \mathrm{H})$, $3.26-3.23(\mathrm{~m}, 1 \mathrm{H}), 2.77-2.73(\mathrm{~m}, 1 \mathrm{H}), 2.67-2.63(\mathrm{~m}, 1 \mathrm{H}), 2.39-2.33(\mathrm{~m}, 1 \mathrm{H}), 2.07-2.02(\mathrm{~m}, 1 \mathrm{H}), 1.91-1.86$ $(\mathrm{m}, 2 \mathrm{H}), 1.83-1.77(\mathrm{~m}, 2 \mathrm{H}), 1.29(\mathrm{~s}, 9 \mathrm{H}) ;{ }^{13} \mathrm{C}$ NMR (151 MHz, CDCl $): \delta=170.7,141.3,128.6(2 \mathrm{C}), 128.5(2 \mathrm{C})$, $126.2,46.5,46.3,44.2,44.0,35.8,33.8,31.7$ (3C) , $26.3,24.2$; HRMS (ESI): calculated for [M+Na] $]^{+}$ $\left(\mathrm{C}_{18} \mathrm{H}_{27} \mathrm{NOSNa} \text { ) m/z: } 328.1711 \text { found [M+Na] }\right]^{+}$328.1708; IR (neat, cm $\left.^{-1}\right):$ 2943, 1734, 1639, 1424, 1275. 
3d: S-(1-Oxo-4-phenyl-1-(pyrrolidin-1-yl)butan-2-yl) ethanethioate<smiles>CC(=O)SC(CCc1ccccc1)C(=O)N1CCCC1</smiles>

The product was prepared according to general procedure E. Purification by flash column chromatography (EtOAc: heptane $=1: 3-1: 1$ ) yielded the product as a yellow oil $(55.9 \mathrm{mg}, 96 \%$ ).

${ }^{1}{ }_{H}$ NMR $\left(600 \mathrm{MHz}, \mathrm{CDCl}_{3}\right): \delta=7.30-7.28(\mathrm{~m}, 2 \mathrm{H}), 7.20-7.17(\mathrm{~m}, 3 \mathrm{H}), 4.29(\mathrm{t}, J=7.5 \mathrm{~Hz}, 1 \mathrm{H}), 3.50-3.41(\mathrm{~m}$, $3 \mathrm{H}), 3.20-3.18(\mathrm{~m}, 1 \mathrm{H}), 2.70-2.68(\mathrm{t}, J=7.7 \mathrm{~Hz}, 2 \mathrm{H}), 2.35(\mathrm{~s}, 3 \mathrm{H}), 2.30-2.27(\mathrm{~m}, 1 \mathrm{H}), 2.07-2.01(\mathrm{~m}, 1 \mathrm{H}), 1.91$ $-1.86(\mathrm{~m}, 2 \mathrm{H}), 1.85-1.78(\mathrm{~m}, 2 \mathrm{H}) ;{ }^{13} \mathrm{C}$ NMR (151 MHz, CDCl 3 ): $\delta=195.2,169.0,140.8,128.5(4 \mathrm{C}), 126.3,46.6$ , 46.3 , 44.4 , 34.1 , 33.3 , $30.4,26.1$, 24.4; HRMS (ESI): calculated for $[\mathrm{M}+\mathrm{Na}]^{+}\left(\mathrm{C}_{16} \mathrm{H}_{21} \mathrm{NO}_{2} \mathrm{SNa}\right) \mathrm{m} / \mathrm{z}: 314.1191$ found [M+Na] $]^{+}$: 314.1182; IR (neat, $\mathrm{cm}^{-1}$ ): 2950, 2926, 2874, 1689, 1639, 1428, 1353, 1128.

\section{3e: 2-((3-Oxobutan-2-yl)thio)-4-phenyl-1-(pyrrolidin-1-yl)butan-1-one}<smiles>CC(=O)C(C)SC(CCc1ccccc1)C(=O)N1CCCC1</smiles>

The product was prepared according to general procedure E. Purification by flash column chromatography (EtOAc: heptane $=1: 4-2: 1$ ) yielded the product as a yellow oil $(45.9 \mathrm{mg}, 72 \%$, d.r. 1:1.3 - calculated from crude NMR).

${ }^{1} \mathrm{H}$ NMR (600 MHz, $\left.\mathrm{CDCl}_{3}\right)$ : (Diastereoisomers present) $\delta=7.29-7.27(\mathrm{~m}, 2 \mathrm{H}), 7.20-7.15(\mathrm{~m}, 3 \mathrm{H}), 3.71(\mathrm{dd}, \mathrm{J}=$ $14.4,7.2 \mathrm{~Hz}, 0.6 \mathrm{H}, \mathrm{d} 2), 3.63-3.57(\mathrm{~m}, 0.4 \mathrm{H}, \mathrm{d} 1), 3.56-3.52(\mathrm{~m}, 0.6 \mathrm{H}, \mathrm{d} 2), 3.48-3.45(\mathrm{~m}, 2+0.4 \mathrm{H}), 3.31-3.28$ $(\mathrm{m}, 0.4 \mathrm{H}, \mathrm{d} 1), 3.28-3.25(\mathrm{~m}, 0.6 \mathrm{H}, \mathrm{d} 2), 3.09-3.06(\mathrm{~m}, 1 \mathrm{H}), 2.75-2.66(\mathrm{~m}, 2 \mathrm{H}), 2.35-2.31(\mathrm{~m}, 1 \mathrm{H}), 2.27(\mathrm{~s}$, $1.3 \mathrm{H}, \mathrm{d} 1), 2.20(\mathrm{~s}, 1.7 \mathrm{H}, \mathrm{d} 2), 2.02(\mathrm{dq}, J=15.0,7.3 \mathrm{~Hz}, 1 \mathrm{H}), 1.94-1.88(\mathrm{~m}, 4 \mathrm{H}), 1.38(\mathrm{~d}, J=7.2 \mathrm{~Hz}, 1.7 \mathrm{H}, \mathrm{d} 2), 1.31$ $(\mathrm{d}, J=7.1 \mathrm{~Hz}, 1.3 \mathrm{H}, \mathrm{d} 1) ;{ }^{13} \mathrm{C}$ NMR (151 MHz, CDCl 3 ): $\delta=206.6$ (d2) , 206.3 (d1), 169.0 (d1) , 168.7 (d2) , 140.7 , $128.4(2 \mathrm{C}), 126.2(2 \mathrm{C}), 126.1,47.1,46.1,46.1,46.1,44.4$ (d2) , 43.9 (d1), 33.9 , $33.6,33.2,26.2,26.0$ (d1) , 26.0 (d2) , 24.3 (d1) , 24.2 (d2) , 17.5 (d1) , 17.0 (d2); HRMS (ESI): calculated for [M+Na] $]^{+}\left(\mathrm{C}_{18} \mathrm{H}_{25} \mathrm{NO}_{2} \mathrm{SNa}\right) \mathrm{m} / \mathrm{z}$ : 342.1504 found $[\mathrm{M}+\mathrm{Na}]^{+}:$342.1497; IR (neat, $\mathrm{cm}^{-1}$ ): 2969, 2927, 2873, 1707, 1633, 1495, 1430, 1352. 


\section{3f: 4-Phenyl-2-(pyrimidin-2-ylthio)-1-(pyrrolidin-1-yl)butan-1-one}<smiles>O=C(C(CCc1ccccc1)Sc1ncccn1)N1CCCC1</smiles>

The product was prepared according to general procedure E. Purification by flash column chromatography (EtOAc: heptane $=1: 3-3: 1$ ) yielded the product as a yellow oil $(49.3 \mathrm{mg}, 81 \%)$.

${ }^{1} \mathrm{H}$ NMR $\left(600 \mathrm{MHz}, \mathrm{CDCl}_{3}\right): \delta=8.52(\mathrm{~d}, J=4.8 \mathrm{~Hz}, 2 \mathrm{H}), 7.32(\mathrm{~d}, J=7.5 \mathrm{~Hz}, 2 \mathrm{H}), 7.28-7.23(\mathrm{~m}, 3 \mathrm{H}), 7.02(\mathrm{t}, J=4.8$ $H z, 1 H), 4.76-4.73(m, 1 H), 3.68-3.64(m, 1 H), 3.61-3.51(m, 2 H), 3.33-3.31(m, 1 H), 2.91-2.78(m, 2 H)$, $2.55-2.47(\mathrm{~m}, 1 \mathrm{H}), 2.35-2.23(\mathrm{~m}, 1 \mathrm{H}), 1.98-1.92(\mathrm{~m}, 2 \mathrm{H}), 1.92-1.88(\mathrm{~m}, 2 \mathrm{H}) ;{ }^{13} \mathrm{C} \mathrm{NMR}\left(151 \mathrm{MHz}, \mathrm{CDCl}_{3}\right):=$ $171.7,169.3,157.4(2 \mathrm{C}), 141.2,128.6$ (2C) $, 128.6,128.4,126.1,116.8,46.6,46.3$, 45.6 , 34.1 , 33.4 , 26.2 , 24.4; HRMS (ESI): calculated for $[\mathrm{M}+\mathrm{Na}]^{+}\left(\mathrm{C}_{18} \mathrm{H}_{21} \mathrm{~N}_{3} \mathrm{OSNa}\right) \mathrm{m} / \mathrm{z}: 350.1303$ found $[\mathrm{M}+\mathrm{Na}]^{+}$: 350.1301 ; IR (neat, cm $\left.{ }^{1}\right): 3112,3018,2929,2855,1712,1564,1547,1431,1379,1186,748$.

\section{3g: Ethyl 2-((1-oxo-4-phenyl-1-(pyrrolidin-1-yl)butan-2-yl)thio)acetate}<smiles>CCOC(=O)CSC(CCc1ccccc1)C(=O)N1CCCC1</smiles>

The product was prepared according to general procedure E. Purification by flash column chromatography (EtOAc: heptane $=1: 3-2: 3$ ) yielded the product as a yellow oil $(60.3 \mathrm{mg}, 90 \%)$.

${ }^{1} \mathrm{H}$ NMR $\left(600 \mathrm{MHz}, \mathrm{CDCl}_{3}\right): \delta=7.28-7.26(\mathrm{~m}, 2 \mathrm{H}), 7.19-7.16(\mathrm{~m}, 3 \mathrm{H}), 4.14(\mathrm{q}, J=7.2 \mathrm{~Hz}, 2 \mathrm{H}), 3.58-3.55(\mathrm{~m}$, $1 \mathrm{H}), 3.48-3.46(\mathrm{~m}, 2 \mathrm{H}) 3.44-3.34(\mathrm{~m}, 1 \mathrm{H}), 3.34-3.33(\mathrm{~m}, 2 \mathrm{H}), 3.11-3.08(\mathrm{~m}, 1 \mathrm{H}), 2.78-2.75(\mathrm{~m}, 1 \mathrm{H}), 2.69-$ $2.65(\mathrm{~m}, 1 \mathrm{H}), 2.37-2.34(\mathrm{~m}, 1 \mathrm{H}), 2.06-2.03(\mathrm{~m}, 1 \mathrm{H}), 1.93-1.80(\mathrm{~m}, 4 \mathrm{H}), 1.25(\mathrm{t}, J=7.2 \mathrm{~Hz}, 3 \mathrm{H}) ;{ }^{13} \mathrm{C}$ NMR $(151$ $\left.\mathrm{MHz}, \mathrm{CDCl}_{3}\right): \delta=170.5,168.5,141.1,128.6(2 \mathrm{C}), 128.5(2 \mathrm{C}), 126.2,61.5,46.3,46.2,43.6,33.3,33.0,31.2$ , 26.1 , $24.4,14.2$; HRMS (ESI): calculated for $[\mathrm{M}+\mathrm{Na}]^{+}\left(\mathrm{C}_{18} \mathrm{H}_{25} \mathrm{NO}_{3} \mathrm{SNa}\right) \mathrm{m} / \mathrm{z}: 358.1453$ found [M+Na] $]^{+}: 358.1448$; IR (neat, cm ${ }^{-1}$ ): 2971, 2927, 2874, 1734, 1637, 1432. 


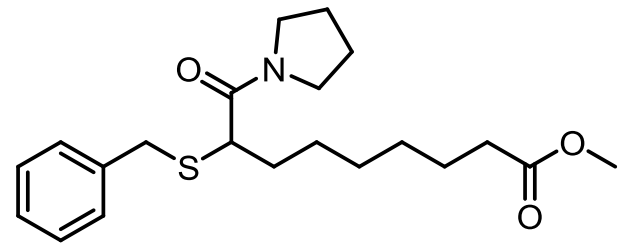

The product was prepared according to general procedure E. Purification by flash column chromatography (EtOAc: heptane $=1: 4-2: 3$ ) yielded the product as a yellow oil $(61.0 \mathrm{mg}, 81 \%)$.

${ }^{1} \mathrm{H}$ NMR $\left(600 \mathrm{MHz}, \mathrm{CDCl}_{3}\right): \delta=7.27-7.23(\mathrm{~m}, 4 \mathrm{H}), 7.18-7.16(\mathrm{~m}, 1 \mathrm{H}), 3.77-3.72(\mathrm{~m}, 2 \mathrm{H}), 3.61(\mathrm{~s}, 3 \mathrm{H}), 3.41-$ $3.39(\mathrm{~m}, 1 \mathrm{H}), 3.37-3.34(\mathrm{~m}, 1 \mathrm{H}), 3.26-3.24(\mathrm{~m}, 1 \mathrm{H}), 3.17-3.13(\mathrm{~m}, 2 \mathrm{H}), 2.25-2.22(\mathrm{~m}, 2 \mathrm{H}), 1.97-1.95(\mathrm{~m}$, $1 \mathrm{H}), 1.82-1.80(\mathrm{~m}, 2 \mathrm{H}), 1.76-1.74(\mathrm{~m}, 2 \mathrm{H}), 1.67-1.65(\mathrm{~m}, 1 \mathrm{H}), 1.56-1.53(\mathrm{~m}, 2 \mathrm{H}), 1.28-1.21(\mathrm{~m}, 6 \mathrm{H}) ;{ }^{13} \mathrm{C}$ NMR (151 MHz, $\left.\mathrm{CDCl}_{3}\right): \delta=174.3,169.3,138.4,129.1$ (2C) , 128.5 (2C) , 127.1 , $51.6,46.2,46.1,45.2,34.1$, $34.0,31.8,29.1,29.0,27.4,26.1,24.9,24.3 ;$ HRMS (ESI): calculated for [M+Na] $]^{+}\left(\mathrm{C}_{21} \mathrm{H}_{31} \mathrm{NO}_{3} \mathrm{SNa}\right) \mathrm{m} / \mathrm{z}: 400.1922$ found $[\mathrm{M}+\mathrm{Na}]^{+}:$400.1912; IR (neat, $\mathrm{cm}^{-1}$ ): 2930, 2876, 1736, 1639, 1431.

3i: 2-(Benzylthio)-1-(pyrrolidin-1-yl)undecane-1,10-dione<smiles>CC(=O)CCCCCCCC(SCc1ccccc1)C(=O)N1CCCC1</smiles>

The product was prepared according to general procedure E. Purification by flash column chromatography (EtOAc: heptane $=1: 4-1: 1$ ) yielded the product as a yellow oil $(70.5 \mathrm{mg}, 94 \%)$.

${ }^{1} \mathrm{H}$ NMR $\left(600 \mathrm{MHz}, \mathrm{CDCl}_{3}\right): \delta=7.28-7.23(\mathrm{~m}, 4 \mathrm{H}), 7.19-7.17(\mathrm{~m}, 1 \mathrm{H}), 3.75-3.74(\mathrm{~m}, 2 \mathrm{H}), 3.42-3.34(\mathrm{~m}, 2 \mathrm{H})$, $3.26-3.24(\mathrm{~m}, 1 \mathrm{H}), 3.18-3.14(\mathrm{~m}, 2 \mathrm{H}), 2.38-2.36(\mathrm{~m}, 2 \mathrm{H}), 2.09(\mathrm{~s}, 3 \mathrm{H}), 1.97-1.94(\mathrm{~m}, 1 \mathrm{H}), 1.84-1.77(\mathrm{~m}, 2 \mathrm{H})$, $1.76-1.73(\mathrm{~m}, 2 \mathrm{H}), 1.69-1.65(\mathrm{~m}, 1 \mathrm{H}), 1.53-1.49(\mathrm{~m}, 2 \mathrm{H}), 1.27-1.22(\mathrm{~m}, 8 \mathrm{H}) ;{ }^{13} \mathrm{C} \mathrm{NMR}\left(151 \mathrm{MHz}, \mathrm{CDCl}_{3}\right): \delta=$ 209.5 , 169.4 , 138.3 , $129.1(2 \mathrm{C}), 128.5$ (2C) , 127.1 , 46.2 , 46.1 , 45.2 , 43.9 , 34.0 , 31.9 , 30.0 , 29.3 , 29.3 , 29.2 , $27.6,26.1,24.3,23.9$; HRMS (ESI): calculated for $[\mathrm{M}+\mathrm{Na}]^{+}\left(\mathrm{C}_{22} \mathrm{H}_{33} \mathrm{NO}_{2} \mathrm{SNa}\right) \mathrm{m} / \mathrm{z}: 398.2130$ found $[\mathrm{M}+\mathrm{Na}]^{+}$: 398.2121; IR (neat, $\mathbf{c m}^{-1}$ ): 2973, 2855, 1713, 1638, 1493, 1427. 


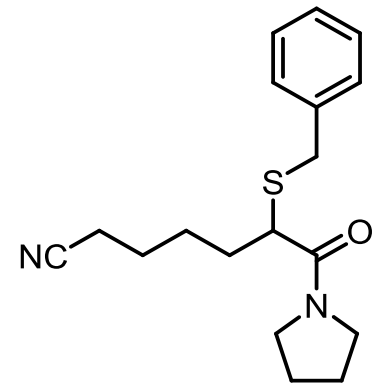

The product was prepared according to general procedure E. Purification by flash column chromatography (EtOAc: heptane = 1:2-20:1) yielded the product as a yellow oil $(52.5 \mathrm{mg}, 83 \%)$.)

${ }^{1} \mathrm{H}$ NMR $\left(600 \mathrm{MHz}, \mathrm{CDCl}_{3}\right): \delta=7.27-7.25(\mathrm{~m}, 4 \mathrm{H}), 7.22-7.18(\mathrm{~m}, 1 \mathrm{H}), 3.77-3.71(\mathrm{~m}, 2 \mathrm{H}), 3.41-3.34(\mathrm{~m}, 1 \mathrm{H})$, $3.33-3.30(\mathrm{~m}, 2 \mathrm{H}), 3.16-3.11(\mathrm{~m}, 2 \mathrm{H}), 2.29-2.22(\mathrm{~m}, 2 \mathrm{H}), 1.99-1.94(\mathrm{~m}, 1 \mathrm{H}), 1.85-1.79(\mathrm{~m}, 2 \mathrm{H}), 1.77-1.72$ (m, 2H), $1.70-1.65(\mathrm{~m}, 1 \mathrm{H}), 1.60-1.55(\mathrm{~m}, 2 \mathrm{H}), 1.49-1.44(\mathrm{~m}, 1 \mathrm{H}), 1.40-1.35(\mathrm{~m}, 1 \mathrm{H}) ;{ }^{13} \mathrm{C} \mathbf{~ N M R}(\mathbf{1 5 1} \mathbf{M H z}$, $\left.\mathrm{CDCl}_{3}\right): \delta=168.7,138.2,129.1(2 \mathrm{C}), 128.5(2 \mathrm{C}), 127.2,119.7,46.2,46.1,44.9,33.9,31.0,26.7,26.1,25.2$ , 24.3 , 17.1; HRMS (ESI): calculated for $[\mathrm{M}+\mathrm{Na}]^{+}\left(\mathrm{C}_{18} \mathrm{H}_{24} \mathrm{~N}_{2} \mathrm{ONa}\right) \mathrm{m} / \mathrm{z}: 339.1507$ found $[\mathrm{M}+\mathrm{Na}]^{+}: 339.1419$; IR (neat, $\mathrm{cm}^{-1}$ ): 3061, 3028, 2931, 2872, 1634, 1493, 1429.

\section{3k: 2-(Benzylthio)-6-chloro-1-(pyrrolidin-1-yl)hexan-1-one}<smiles>O=C(C(CCCCCl)SCc1ccccc1)N1CCCC1</smiles>

The product was prepared according to general procedure E. Purification by flash column chromatography (EtOAc: heptane $=1: 4-1: 1$ ) yielded the product as a yellow oil $(51.3 \mathrm{mg}, 79 \%)$.

${ }^{1} \mathrm{H}$ NMR $\left(600 \mathrm{MHz}, \mathrm{CDCl}_{3}\right): \delta=7.32-7.28(\mathrm{~m}, 4 \mathrm{H}), 7.23-7.21(\mathrm{~m}, 1 \mathrm{H}), 3.82-3.76(\mathrm{~m}, 2 \mathrm{H}), 3.54-3.31(\mathrm{~m}, 5 \mathrm{H})$, $3.21-3.17(\mathrm{~m}, 2 \mathrm{H}), 2.06-2.00(\mathrm{~m}, 1 \mathrm{H}), 1.90-1.84(\mathrm{~m}, 2 \mathrm{H}), 1.79-1.68(\mathrm{~m}, 5 \mathrm{H}), 1.56-1.49(\mathrm{~m}, 1 \mathrm{H}), 1.45-1.38$ (m, 1H); ${ }^{13} \mathrm{C}$ NMR (151 MHz, $\left.\mathrm{CDCl}_{3}\right): \delta=169.0,138.3,129.2$ (2C) , 128.5 (2C) , $127.2,46.2,46.1,45.0,45.0$, 34.0 , $32.4,31.1,26.1,25.0$, 24.3; HRMS (ESI): calculated for $[\mathrm{M}+\mathrm{Na}]^{+}\left(\mathrm{C}_{17} \mathrm{H}_{24} \mathrm{CINOSNa}\right) \mathrm{m} / \mathrm{z}: 348.1165$ found $[\mathrm{M}+\mathrm{Na}]^{+}:$348.1152; IR (neat, $\mathrm{cm}^{-1}$ ): 2951, 2871, 1634, 1493, 1428. 


\section{I: 2-(benzylthio)-1-(pyrrolidin-1-yl)undec-10-en-1-one}<smiles>C=CCCCCCCCC(SCc1ccccc1)C(=O)N1CCCC1</smiles>

The product was prepared according to general procedure E. Purification by flash column chromatography (EtOAc: heptane $=1: 4-1: 1$ ) yielded the product as a yellow oil $(58.8 \mathrm{mg}, 82 \%)$.

${ }^{1} \mathrm{H}$ NMR $\left(600 \mathrm{MHz}, \mathrm{CDCl}_{3}\right): \delta=7.27-7.22(\mathrm{~m}, 4 \mathrm{H}), 7.18-7.16(\mathrm{~m}, 1 \mathrm{H}) 5.79-5.72(\mathrm{~m}, 1 \mathrm{H}), 4.91(\mathrm{~m}, 2 \mathrm{H}), 3.75(\mathrm{~s}$, $2 \mathrm{H}), 3.43-3.33(\mathrm{~m}, 2 \mathrm{H}), 3.25-3.22(\mathrm{~m}, 1 \mathrm{H}), 3.18-3.12(\mathrm{~m}, 2 \mathrm{H}), 1.99-1.92(\mathrm{~m}, 3 \mathrm{H}), 1.83-1.77(\mathrm{~m}, 2 \mathrm{H}), 1.76-$ $1.73(\mathrm{~m}, 2 \mathrm{H}), 1.70-1.65(\mathrm{~m}, 1 \mathrm{H}), 1.31-1.21(\mathrm{~m}, 10 \mathrm{H}) ;{ }^{13} \mathrm{C} \mathrm{NMR}\left(151 \mathrm{MHz}, \mathrm{CDCl}_{3}\right): \delta=169.4,139.3,138.4$, $129.1(2 \mathrm{C}), 128.5(2 \mathrm{C}), 127.1$, 114.3 , 46.2 , 46.1 , 45.2 , 34.0 , 33.9 , 31.9 , 29.4 , 29.4 , 29.1 , 29.0 , 27.6 , 26.1 , 24.3 ; HRMS (ESI): calculated for $[\mathrm{M}+\mathrm{Na}]^{+}\left(\mathrm{C}_{22} \mathrm{H}_{33} \mathrm{NOSNa}\right) \mathrm{m} / \mathrm{z}: 382.2181$ found $[\mathrm{M}+\mathrm{Na}]^{+}:$382.2177; IR (neat, $\mathbf{c m}^{-}$ 1): 3027, 2971, 2853, 2362, 1736, 1639, 1425.

\section{3m: 2-(Benzylthio)-1-(pyrrolidin-1-yl)hex-5-yn-1-one}<smiles>C#CCCC(SCc1ccccc1)C(=O)N1CCCC1</smiles>

The product was prepared according to general procedure E. Purification by flash column chromatography (EtOAc: heptane $=1: 4-2: 1$ ) yielded the product as a yellow oil $(45.9 \mathrm{mg}, 80 \%)$.

${ }^{1} \mathrm{H}$ NMR $\left(600 \mathrm{MHz}, \mathrm{CDCl}_{3}\right): \delta=7.34-7.33(\mathrm{~m}, 2 \mathrm{H}), 7.30-7.28(\mathrm{~m}, 2 \mathrm{H}), 7.24-7.21(\mathrm{~m}, 1 \mathrm{H}), 3.83-3.77(\mathrm{~m}, 2 \mathrm{H})$, $3.53-3.51(\mathrm{~m}, 1 \mathrm{H}), 3.46-3.44(\mathrm{~m}, 1 \mathrm{H}), 3.39-3.37(\mathrm{~m}, 1 \mathrm{H}), 3.33-3.31(\mathrm{~m}, 1 \mathrm{H}), 3.25-3.22(\mathrm{~m}, 1 \mathrm{H}), 2.41-2.37$ (m, 1H), $\left.2.27-2.22(\mathrm{~m}, 2 \mathrm{H}), 1.96-1.77(\mathrm{~m}, 6 \mathrm{H}) ;{ }^{13} \mathrm{C} \mathrm{NMR} \mathrm{(151} \mathrm{MHz,} \mathrm{CDCl} 3\right): \delta=168.6,138.4,129.3(2 \mathrm{C}), 128.6$ (2C) $, 127.2,69.4,46.1,46.1,43.5,34.1,30.5,26.1$, $24.4,16.5$; (the quaternary carbon of the alkyne is not relaxing) HRMS (ESI): calculated for $[\mathrm{M}+\mathrm{Na}]^{+}\left(\mathrm{C}_{17} \mathrm{H}_{21} \mathrm{NOSNa}\right) \mathrm{m} / \mathrm{z}: 310.1242$ found $[\mathrm{M}+\mathrm{Na}]^{+}: 310.1227$; IR (neat, cm $\left.^{-1}\right): 3028,2931,2872,1738,1634,1493$. 


\section{3n: 2-(Benzylthio)-N,N-dimethyl-4-phenylbutanamide}<smiles>CN(C)C(=O)C(CCc1ccccc1)SCc1ccccc1</smiles>

The product was prepared according to general procedure E. Purification by flash column chromatography (EtOAc: heptane $=1: 4-1: 1)$ yielded the product as a yellow oil $(51.9 \mathrm{mg}, 83 \%)$.

${ }^{1} \mathrm{H}$ NMR $\left(600 \mathrm{MHz}, \mathrm{CDCl}_{3}\right): \delta=7.36-7.26(\mathrm{~m}, 8 \mathrm{H}), 7.22-7.20(\mathrm{~m}, 2 \mathrm{H}), 3.80(\mathrm{~s}, 2 \mathrm{H}), 3.35-3.32(\mathrm{~m}, 1 \mathrm{H}), 3.00(\mathrm{~s}$, 3H), $2.84-2.82(\mathrm{~m}, 1 \mathrm{H}), 2.72(\mathrm{~s}, 3 \mathrm{H}), 2.67-2.64(\mathrm{~m}, 1 \mathrm{H}), 2.53-2.48(\mathrm{~m}, 1 \mathrm{H}) 2.13-2.10(\mathrm{~m}, 1 \mathrm{H}) ;{ }^{13} \mathrm{C}$ NMR (151 $\left.\mathrm{MHz}, \mathrm{CDCl}_{3}\right): \delta=170.4,141.3,138.1,129.1(2 \mathrm{C}), 128.7(2 \mathrm{C}), 128.6(2 \mathrm{C}), 128.5(2 \mathrm{C}), 127.1,126.1,41.4,36.9$ , 36.1 , 34.0 , 33.6 , 33.5; HRMS (ESI): calculated for $[\mathrm{M}+\mathrm{Na}]^{+}\left(\mathrm{C}_{19} \mathrm{H}_{23} \mathrm{NOSNa}\right) \mathrm{m} / \mathrm{z}: 336.1398$ found $[\mathrm{M}+\mathrm{Na}]^{+}$:

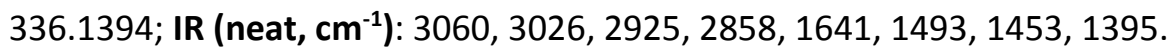

\section{3o: Methyl 8-(benzylthio)-9-(diallylamino)-9-oxononanoate}<smiles>C=CCN(CC=C)C(=O)C(CCCCCCC(=O)OC)SCc1ccccc1</smiles>

The product was prepared according to general procedure E. Purification by column chromatography on silica gel (10-30\% EtOAc in heptane) yielded the product $(30.0 \mathrm{mg}, 37 \%)$ as a pale yellow oil.

${ }^{1} \mathrm{H}$ NMR $\left(400 \mathrm{MHz}, \mathrm{CDCl}_{3}\right): \delta=7.32-7.27(\mathrm{~m}, 4 \mathrm{H}), 7.25-7.20(\mathrm{~m}, 1 \mathrm{H}), 5.80-5.59(\mathrm{~m}, 2 \mathrm{H}), 5.20-4.99(\mathrm{~m}, 4 \mathrm{H})$, $4.27-4.39(\mathrm{~m}, 1 \mathrm{H}), 3.99-3.90(\mathrm{~m}, 1 \mathrm{H}), 3.78(\mathrm{dd}, J=24.8,12.8 \mathrm{~Hz}, 2 \mathrm{H}), 3.67(\mathrm{~s}, 3 \mathrm{H}), 3.65-3.55(\mathrm{~m}, 2 \mathrm{H}), 3.27-$ $3.20(\mathrm{~m}, 1 \mathrm{H}), 2.29(\mathrm{t}, J=7.4 \mathrm{~Hz}, 2 \mathrm{H}), 2.12-2.00(\mathrm{~m}, 1 \mathrm{H}), 1.77-1.66(\mathrm{~m}, 1 \mathrm{H}), 1.64-1.54(\mathrm{~m}, 3 \mathrm{H}), 1.33-1.22(\mathrm{~m}$, 5H); $\left.{ }^{13} \mathrm{C} \mathrm{NMR} \mathrm{(100} \mathrm{MHz,} \mathrm{CDCl} 3\right): \delta=174.4,170.9,138.1,133.6,133.3,129.3$ (2C), 128.7 (2C), 127.2, 117.1, 116.2, 51.6, 49.2, 48.5, 43.0, 34.2, 33.9, 32.1, 29.2, 29.1, 27.6, 25.0; HRMS (ESI): calculated for [M+Na] ${ }^{+}\left(\mathrm{C}_{23} \mathrm{H}_{33} \mathrm{NNaO}_{3} \mathrm{~S}\right)$ 426.2073, found 426.2081; IR (neat, $\mathrm{cm}^{-1}$ ): = 2926, 2855, 1736, 1639, 1453, 1193, 1173, 922, 703. 


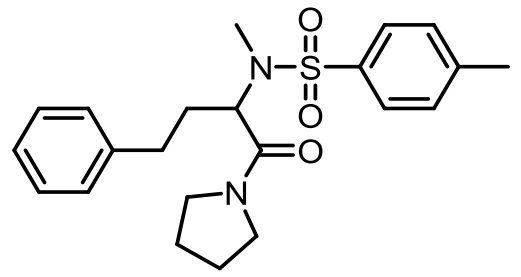

The product was prepared according to general procedure E. Purification by column chromatography on silica gel (EtOAc: heptane $=1: 2)$ yielded the product $(57.0 \mathrm{mg}, 71 \%)$ as a pale yellow liquid.

${ }^{1} \mathrm{H}$ NMR $\left(400 \mathrm{MHz}, \mathrm{CDCl}_{3}\right): \delta=7.58(\mathrm{~d}, J=7.8 \mathrm{~Hz}, 2 \mathrm{H}), 7.32-7.26(\mathrm{~m}, 4 \mathrm{H}), 7.23-7.18(\mathrm{~m}, 1 \mathrm{H}), 7.09(\mathrm{~d}, J=7.8 \mathrm{~Hz}$, $2 \mathrm{H}), 4.59(\mathrm{dd}, J=9.8,6.4 \mathrm{~Hz}, 1 \mathrm{H}), 3.76-3.68(\mathrm{~m}, 1 \mathrm{H}), 3.46-3.21(\mathrm{~m}, 3 \mathrm{H}), 2.92(\mathrm{~s}, 3 \mathrm{H}), 2.64-2.54(\mathrm{~m}, 1 \mathrm{H}), 2.50$ $-2.41(\mathrm{~m}, 1 \mathrm{H}), 2.42(\mathrm{~s}, 3 \mathrm{H}), 2.18-2.09(\mathrm{~m}, 1 \mathrm{H}), 1.98-1.88(\mathrm{~m}, 2 \mathrm{H}), 1.87-1.78(\mathrm{~m}, 2 \mathrm{H}), 1.51-1.40(\mathrm{~m}, 1 \mathrm{H}) ;{ }^{13} \mathrm{C}$ NMR (100 MHz, CDCl $): \delta=167.7,143.6,140.9,136.3,129.8$ (2C), 128.6 (2C), 128.6 (2C), $127.4(2 \mathrm{C}), 126.4,56.5$, 46.6, 46.1, 32.4, 30.5, 29.6, 26.3, 24.3, 21.7; HRMS (ESI): calculated for $[\mathrm{M}+\mathrm{Na}]^{+}\left(\mathrm{C}_{22} \mathrm{H}_{28} \mathrm{~N}_{2} \mathrm{O}_{3} \mathrm{SNa}\right) \mathrm{m} / \mathrm{z}$ : 423.1713, found 423.1716; IR (neat; $\mathrm{cm}^{-1}$ ): 3059, 3026, 2950, 2874, 1740, 1639, 1441, 1335, 1156, 1087, 933, 814, 696, 651.

4b: N-Isopropyl-4-methyl-N-(1-oxo-4-phenyl-1-(pyrrolidin-1-yl)butan-2-yl)benzenesulfonamide

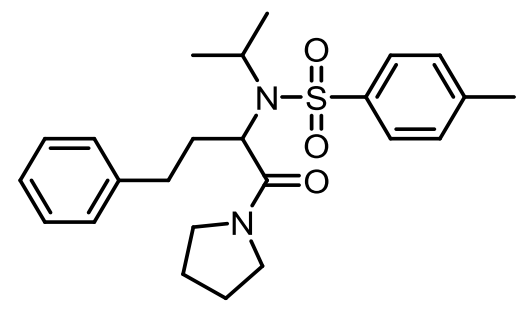

The product was prepared according to general procedure E. Purification by column chromatography on silica gel (EtOAc: heptane $=1: 3)$ yielded the product $(54.6 \mathrm{mg}, 64 \%)$ as a pale yellow solid.

${ }^{1} \mathrm{H}$ NMR $\left(400 \mathrm{MHz}, \mathrm{CDCl}_{3}\right): \delta=7.60(\mathrm{~d}, J=7.8 \mathrm{~Hz}, 2 \mathrm{H}), 7.31-7.17(\mathrm{~m}, 5 \mathrm{H}), 7.04(\mathrm{~d}, J=7.8 \mathrm{~Hz}, 2 \mathrm{H}), 4.40(\mathrm{dd}, J=$ 10.3, 4.1 Hz, 1H), $4.15-4.05(\mathrm{~m}, 1 \mathrm{H}), 3.72-3.64(\mathrm{~m}, 1 \mathrm{H}), 3.56-3.47(\mathrm{~m}, 1 \mathrm{H}), 3.47-3.38(\mathrm{~m}, 1 \mathrm{H}), 3.24-3.16$ (m, 1H), $2.65-2.56(\mathrm{~m}, 1 \mathrm{H}), 2.42(\mathrm{~s}, 3 \mathrm{H}), 2.41-2.24(\mathrm{~m}, 2 \mathrm{H}), 1.94-1.78(\mathrm{~m}, 4 \mathrm{H}), 1.52-1.42(\mathrm{~m}, 1 \mathrm{H}), 1.33(\mathrm{~d}, J$ $=6.9 \mathrm{~Hz}, 6 \mathrm{H}) ;{ }^{13} \mathrm{C} \mathrm{NMR}\left(100 \mathrm{MHz}, \mathrm{CDCl}_{3}\right): \delta=167.5,143.1,141.0,139.6,129.6(2 \mathrm{C}), 128.7(2 \mathrm{C}), 128.6(2 \mathrm{C}), 127.6$ (2C), 126.4, 57.7, 50.4, 46.5, 46.5, 32.7, 31.2, 26.4, 24.2, 23.3, 22.4, 21.6; HRMS (ESI): calculated for [M+Na] ${ }^{+}$ $\left(\mathrm{C}_{24} \mathrm{H}_{32} \mathrm{~N}_{2} \mathrm{O}_{3} \mathrm{SNa}\right.$ ) m/z: 451.2026, found 451.2022; IR (neat, $\mathrm{cm}^{-1}$ ): 3026, 2970, 2873, 1637, 1599, 1439, 1328, $1151,1085,965,936,815,698,597$. 


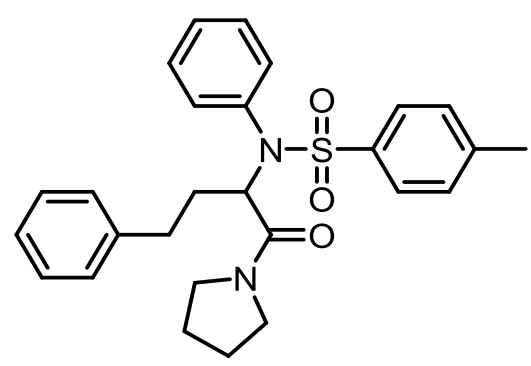

The product was prepared according to general procedure E. Purification by column chromatography on silica gel (EtOAc: heptane $=1: 3)$ yielded the product $(69.7 \mathrm{mg}, 75 \%)$ as a white solid.

${ }^{1} \mathrm{H}$ NMR $\left(400 \mathrm{MHz}, \mathrm{CDCl}_{3}\right): \delta=7.36(\mathrm{~d}, J=8.6 \mathrm{~Hz}, 2 \mathrm{H}), 7.32-7.27(\mathrm{~m}, 2 \mathrm{H}), 7.26-7.19(\mathrm{~m}, 5 \mathrm{H}), 7.18-7.10(\mathrm{~m}$, $3 \mathrm{H}), 7.98-7.93(\mathrm{~m}, 2 \mathrm{H}), 5.04-4.96(\mathrm{~m}, 1 \mathrm{H}), 3.88-3.78(\mathrm{~m}, 1 \mathrm{H}), 3.40-3.22(\mathrm{~m}, 3 \mathrm{H}), 2.59-2.42(\mathrm{~m}, 2 \mathrm{H}), 2.31$ (s, 3H), $2.03-1.75(\mathrm{~m}, 5 \mathrm{H}), 1.74-1.64(\mathrm{~m}, 1 \mathrm{H}) ;{ }^{13} \mathrm{C}$ NMR (100 MHz, CDCl $): \delta=167.8,143.5,140.9,137.0$, $136.0,132.4(2 \mathrm{C}), 129.3(2 \mathrm{C}), 128.8$ (2C), 128.7 (2C) 128.7 (2C) 128.6 (2C) $127.9,126.4,59.1,46.5,46.3,32.6$ , 32.4 , 26.5 , 24.3 , 21.7; HRMS (ESI): calculated for $[\mathrm{M}+\mathrm{H}]^{+}\left(\mathrm{C}_{27} \mathrm{H}_{31} \mathrm{~N}_{2} \mathrm{O}_{3} \mathrm{~S}\right) \mathrm{m} / \mathrm{z}$ : 463.2050, found 463.2046; IR (neat, $\mathrm{cm}^{-1}$ ): 3061, 3026, 2951, 2874, 2242, 1643, 1597, 1491, 1439, 1342, 1159, 909, 729, 697, 657.

4d: N-(4-Methoxyphenyl)-4-methyl-N-(1-oxo-4-phenyl-1-(pyrrolidin-1-yl)butan-2-yl)benzenesulfonamide

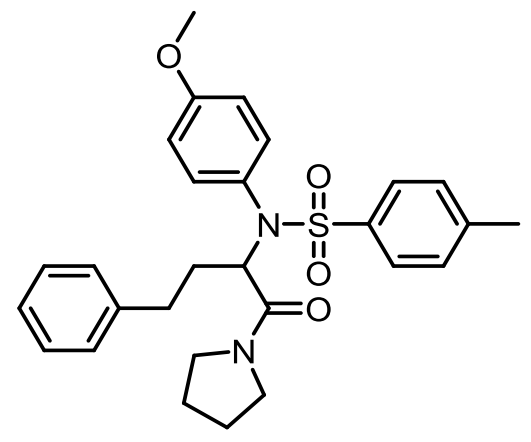

The product was prepared according to general procedure E. Purification by column chromatography on silica gel (EtOAc: heptane $=1: 2)$ yielded the product $(70.4 \mathrm{mg}, 72 \%)$ as a pale yellow solid.

${ }^{1} \mathrm{H}$ NMR $\left(400 \mathrm{MHz}, \mathrm{CDCl}_{3}\right): \delta=7.40(\mathrm{~d}, J=8.3 \mathrm{~Hz}, 2 \mathrm{H}), 7.31-7.24(\mathrm{~m}, 2 \mathrm{H}), 7.23-7.16(\mathrm{~m}, 5 \mathrm{H}), 7.13-7.08(\mathrm{~m}$, 2H), $6.77(\mathrm{~d}, J=9.0 \mathrm{~Hz}, 2 \mathrm{H}), 5.03(\mathrm{dd}, J=8.1,6.4 \mathrm{~Hz}, 1 \mathrm{H}), 3.93-3.85(\mathrm{~m}, 1 \mathrm{H}), 3.78(\mathrm{~s}, 3 \mathrm{H}), 3.45-3.28(\mathrm{~m}, 3 \mathrm{H})$, $2.64-2.48(\mathrm{~m}, 2 \mathrm{H}), 2.39(\mathrm{~s}, 3 \mathrm{H}), 2.07-1.82(\mathrm{~m}, 5 \mathrm{H}), 1.76-1.66(\mathrm{~m}, 1 \mathrm{H}) ;{ }^{13} \mathrm{C} \mathrm{NMR}\left(100 \mathrm{MHz}, \mathrm{CDCl}_{3}\right): \delta=167.9$, $159.7,143.4,141.0,137.0,133.5(2 \mathrm{C}), 129.2(2 \mathrm{C}), 128.6(2 \mathrm{C}), 128.6(2 \mathrm{C}), 128.2,127.9(2 \mathrm{C}), 126.3,113.9(2 \mathrm{C})$, 59.0 , 55.4 , 46.4 , 46.2 , 32.5 , 32.4 , 26.5 , 24.3 , 21.6; HRMS (ESI): calculated for $[\mathrm{M}+\mathrm{H}]^{+}\left(\mathrm{C}_{28} \mathrm{H}_{33} \mathrm{~N}_{2} \mathrm{O}_{4} \mathrm{~S}\right) \mathrm{m} / \mathrm{z}$ : 493.2156, found 493.2164; IR (neat, $\mathrm{cm}^{-1}$ ): = 3062, 3026, 2952, 2874, 2240, 1644, 1602, 1505, 1439, 1341, 1249, $1158,914,727,589$. 
<smiles>Cc1ccc(S(=O)(=O)N(Cc2ccccc2)C(CCc2ccccc2)C(=O)N2CCCC2)cc1</smiles>

The product was prepared according to general procedure E. Purification by column chromatography on silica gel (EtOAc: heptane $=1: 2)$ yielded the product $(67.0 \mathrm{mg}, 70 \%)$ as a pale yellow solid.

${ }^{1} \mathrm{H}$ NMR $\left(400 \mathrm{MHz}, \mathrm{CDCl}_{3}\right): \delta=7.62(\mathrm{~d}, J=8.1 \mathrm{~Hz}, 2 \mathrm{H}), 7.44(\mathrm{~d}, J=7.1 \mathrm{~Hz}, 2 \mathrm{H}), 7.32-7.14(\mathrm{~m}, 8 \mathrm{H}), 6.98-6.93(\mathrm{~m}$, 2H), $4.87(\mathrm{~d}, J=16.1 \mathrm{~Hz}, 1 \mathrm{H}), 4.53(\mathrm{dd}, J=9.1,4.8 \mathrm{~Hz}, 1 \mathrm{H}), 4.33(\mathrm{~d}, J=16.1 \mathrm{~Hz}, 1 \mathrm{H}), 3.55-3.46(\mathrm{~m}, 1 \mathrm{H}), 3.20-$ $3.11(\mathrm{~m}, 2 \mathrm{H}), 2.68-2.59(\mathrm{~m}, 1 \mathrm{H}), 2.55-2.47(\mathrm{~m}, 1 \mathrm{H}), 2.43(\mathrm{~s}, 3 \mathrm{H}), 2.37-2.27(\mathrm{~m}, 1 \mathrm{H}), 2.24-2.13(\mathrm{~m}, 1 \mathrm{H}), 1.80$ - $1.61(\mathrm{~m}, 3 \mathrm{H}), 1.53$ - $\left.1.42(\mathrm{~m}, 1 \mathrm{H}), 1.41-1.31(\mathrm{~m}, 1 \mathrm{H}) ;{ }^{13} \mathrm{C} \mathrm{NMR} \mathrm{(100} \mathrm{MHz,} \mathrm{CDCl} 3\right): \delta=166.7,143.7,141.1$, 137.9 , 137.7 , 129.8 (2C), 128.7 (2C), 128.5 (2C), 128.5 (2C), 128.2 (2C), 127.5 (2C), $127.4,126.3,57.2$, 48.6 ， 46.1 , 45.9 , 32.7 , 30.2 , 26.2 , 23.9 , 21.7; HRMS (ESI): calculated for $[\mathrm{M}+\mathrm{H}]^{+}\left(\mathrm{C}_{28} \mathrm{H}_{33} \mathrm{~N}_{2} \mathrm{O} 3{ }_{3} \mathrm{~S}\right) \mathrm{m} / \mathrm{z}: 477.2206$, found 477.2201; IR (neat, cm cm $^{-1}$ : 3061, 3027, 2948, 2874, 1642, 1599, 1494, 1435, 1335, 1156, 1093, 698, 654.

\section{4f: N-Methyl-4-nitro-N-(1-oxo-4-phenyl-1-(pyrrolidin-1-yl)butan-2-yl)benzenesulfonamide}

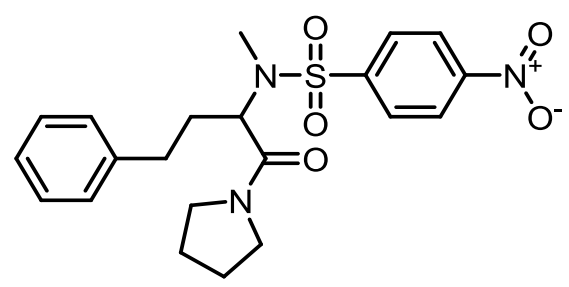

The product was prepared according to general procedure E. Purification by column chromatography on silica gel (EtOAc: heptane $=1: 2)$ yielded the product $(82.3 \mathrm{mg}, 82 \%)$ as a pale yellow liquid.

${ }^{1} \mathrm{H}$ NMR $\left(400 \mathrm{MHz}, \mathrm{CDCl}_{3}\right): \delta=8.30(\mathrm{~d}, J=9.0 \mathrm{~Hz}, 2 \mathrm{H}), 7.82(\mathrm{~d}, J=9.0 \mathrm{~Hz}, 2 \mathrm{H}), 7.35-7.29(\mathrm{~m}, 2 \mathrm{H}), 7.27-7.21(\mathrm{~m}$, 1H), $7.15-7.10(\mathrm{~m}, 2 \mathrm{H}), 4.63(\mathrm{apt}, J=7.5 \mathrm{~Hz}, 1 \mathrm{H}), 3.64-3.57(\mathrm{~m}, 1 \mathrm{H}), 3.41-3.19(\mathrm{~m}, 3 \mathrm{H}), 3.03(\mathrm{~s}, 3 \mathrm{H}), 2.67-$ $2.50(\mathrm{~m}, 2 \mathrm{H}), 2.18-2.07(\mathrm{~m}, 1 \mathrm{H}), 1.98-1.89(\mathrm{~m}, 2 \mathrm{H}), 1.89-1.80(\mathrm{~m}, 2 \mathrm{H}), 1.65-1.55(\mathrm{~m}, 1 \mathrm{H}) ;{ }^{13} \mathrm{C}$ NMR (100 $\left.\mathrm{MHz}, \mathrm{CDCl}_{3}\right): \delta=167.7,150.1,144.8$, $140.4,128.8$ (2C), 128.6 (2C), 128.5 (2C), $126.7,124.3$ (2C), 56.4 , 46.5 , 46.1 , 32.1 , 30.8 , 30.3 , 26.3 , 24.2; HRMS (ESI): calculated for $[\mathrm{M}+\mathrm{Na}]^{+}\left(\mathrm{C}_{21} \mathrm{H}_{25} \mathrm{~N}_{3} \mathrm{O}_{5} \mathrm{SNa}\right) \mathrm{m} / \mathrm{z}: 454.1407$, found 454.1411; IR (neat, cm $^{-1}$ ): = 3103, 3063, 2974, 2876, 1642, 1529, 1448, 1349, 1162, 855, 699, 608. 
4g: 4-Nitro-N-(1-oxo-4-phenyl-1-(pyrrolidin-1-yl)butan-2-yl)benzenesulfonamide

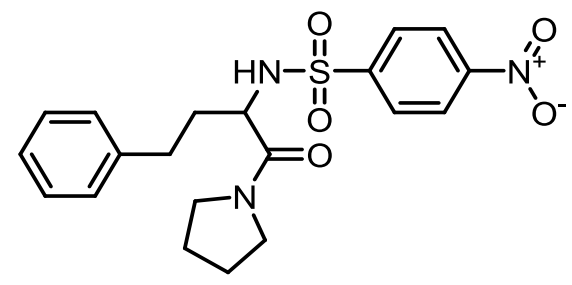

The product was prepared according to general procedure E. Purification by column chromatography on silica gel (EtOAc: heptane $=1: 2$ ) yielded the product $(57.2 \mathrm{mg}, 69 \%)$ as a white solid.

${ }^{1} \mathrm{H}$ NMR $\left(400 \mathrm{MHz}, \mathrm{CDCl}_{3}\right): \delta=8.29(\mathrm{~d}, J=8.8 \mathrm{~Hz}, 2 \mathrm{H}), 7.99(\mathrm{~d}, J=8.8 \mathrm{~Hz}, 2 \mathrm{H}), 7.33-7.25(\mathrm{~m}, 2 \mathrm{H}), 7.24-7.16(\mathrm{~m}$, 3H), 5.86 (bs, 1H), 3.94 (bs, 1H), $3.20-3.11(\mathrm{~m}, 1 \mathrm{H}), 2.99-2.68(\mathrm{~m}, 5 \mathrm{H}), 1.94-1.58(\mathrm{~m}, 6 \mathrm{H}) ;{ }^{13} \mathrm{C} \mathrm{NMR}(100 \mathrm{MHz}$, $\left.\mathrm{CDCl}_{3}\right): \delta=168.5,150.2,145.8,140.5,128.8(2 \mathrm{C}), 128.7(4 \mathrm{C}), 126.6,124.1(2 \mathrm{C}), 54.1,46.1,45.9,35.1,31.3$ , 26.0 , 24.0; HRMS (ESI): calculated for $[\mathrm{M}+\mathrm{Na}]^{+}\left(\mathrm{C}_{20} \mathrm{H}_{23} \mathrm{~N}_{3} \mathrm{O}_{5} \mathrm{SNa}\right) \mathrm{m} / \mathrm{z}: 440.1251$, found 440.1251; IR (neat, $\mathrm{cm}^{-}$ 1): 3104, 2952, 2876, 1629, 1528, 1451, 1348, 1164, 1091, 855, 613.

4h: N-Methyl-2-nitro-N-(1-oxo-4-phenyl-1-(pyrrolidin-1-yl)butan-2-yl)benzenesulfonamide

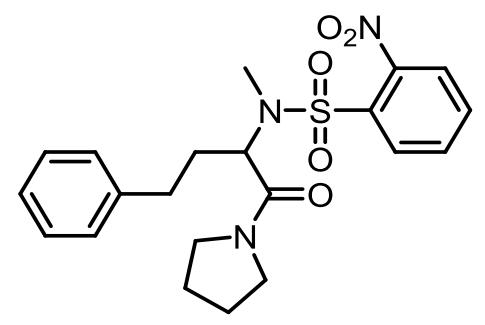

The product was prepared according to general procedure E. Purification by column chromatography on silica gel (EtOAc: heptane $=1: 4-7: 3)$ yielded the product $(69.0 \mathrm{mg}, 80 \%)$ as a pale yellow solid.

${ }^{1} \mathrm{H}$ NMR $\left(400 \mathrm{MHz}, \mathrm{CDCl}_{3}\right): \delta=7.94-7.90(\mathrm{~m}, 1 \mathrm{H}), 7.71-7.60(\mathrm{~m}, 3 \mathrm{H}), 7.30-7.24(\mathrm{~m}, 2 \mathrm{H}), 7.23-7.12(\mathrm{~m}, 3 \mathrm{H})$, $4.75(\mathrm{apt}, J=7.4 \mathrm{~Hz}, 1 \mathrm{H}), 3.70-3.61(\mathrm{~m}, 1 \mathrm{H}), 3.44-3.37(\mathrm{~m}, 2 \mathrm{H}), 3.31-3.23(\mathrm{~m}, 1 \mathrm{H}), 3.14(\mathrm{~s}, 3 \mathrm{H}), 2.71-2.56$ $(\mathrm{m}, 2 \mathrm{H}), 2.21-2.12(\mathrm{~m}, 1 \mathrm{H}), 1.95-1.78(\mathrm{~m}, 5 \mathrm{H}) ;{ }^{13} \mathrm{C}$ NMR (100 MHz, CDCl $): \delta=168.5,148.3,140.8,133.6,132.7$, 131.7, 130.8, 128.6 (2C), 128.5 (2C), 126.4, 124.4, 56.7, 46.6, 46.2, 32.3, 31.1, 31.0, 26.3, 24.2; HRMS (ESI): calculated for $[\mathrm{M}+\mathrm{Na}]^{+}\left(\mathrm{C}_{21} \mathrm{H}_{25} \mathrm{~N}_{3} \mathrm{O}_{5} \mathrm{SNa}\right) \mathrm{m} / \mathrm{z}: 454.1407$, found 454.1412; IR (neat, $\left.\mathrm{cm}^{-1}\right)$ : 3064, 3025, 2951, 2875, $1637,1542,1440,1370,1348,1161,937,851,729,700$. 


\section{4i: N-Methyl-N-(1-oxo-4-phenyl-1-(pyrrolidin-1-yl)butan-2-yl)pyridine-2-sulfonamide}

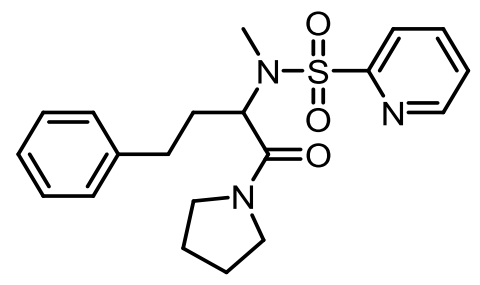

The product was prepared according to general procedure E. Purification by column chromatography on silica gel (EtOAc: heptane $=1: 2)$ yielded the product $(57.0 \mathrm{mg}, 74 \%)$ as a pale yellow liquid.

${ }^{1} \mathrm{H}$ NMR $\left(400 \mathrm{MHz}, \mathrm{CDCl}_{3}\right): \delta=8.66-8.63(\mathrm{~m}, 1 \mathrm{H}), 7.92-7.83(\mathrm{~m}, 2 \mathrm{H}), 7.49-7.43(\mathrm{~m}, 1 \mathrm{H}), 7.31-7.24(\mathrm{~m}, 2 \mathrm{H})$, $7.21-7.12(\mathrm{~m}, 3 \mathrm{H}), 4.81(\mathrm{dd}, J=8.0,6.8 \mathrm{~Hz}, 1 \mathrm{H}), 3.88-3.79(\mathrm{~m}, 1 \mathrm{H}), 3.45-3.32(\mathrm{~m}, 2 \mathrm{H}), 3.30-3.22(\mathrm{~m}, 1 \mathrm{H})$, $3.04(\mathrm{~s}, 3 \mathrm{H}), 2.67-2.52(\mathrm{~m}, 2 \mathrm{H}), 2.20-2.09(\mathrm{~m}, 1 \mathrm{H}), 1.98-1.77(\mathrm{~m}, 5 \mathrm{H}) ;{ }^{13} \mathrm{C} \mathbf{N M R}\left(100 \mathrm{MHz}, \mathrm{CDCl}_{3}\right): \delta=168.2$, 157.2 , 150.1 , 141.0 , 137.9 , 128.6 (2C), 128.6 (2C), 126.7 , 126.3 , 122.8 , 56.9 , 46.6 , 46.1 , 32.3 , 30.9 , 30.8 ， 26.3 , 24.4; HRMS (ESI): calculated for [M+Na] ${ }^{+}\left(\mathrm{C}_{20} \mathrm{H}_{25} \mathrm{~N}_{3} \mathrm{O}_{3} \mathrm{SNa}\right) \mathrm{m} / \mathrm{z}: 410.1509$, found 410.1507; IR (neat, $\left.\mathbf{c m}^{-1}\right)$ : 3025, 2951, 2874, 1640, 1577, 1449, 1428, 1340, 1172, 1083, 937, 778, 744, 700, 589.

4j: N-Methyl-N-(1-oxo-4-phenyl-1-(pyrrolidin-1-yl)butan-2-yl)naphthalene-2-sulfonamide

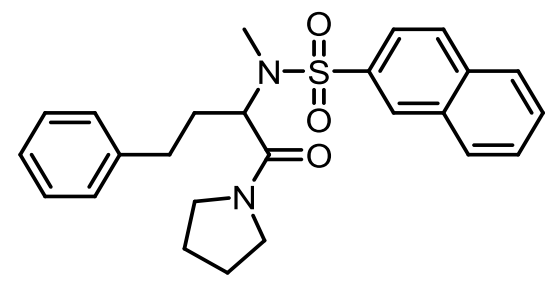

The product was prepared according to general procedure E. Purification by column chromatography on silica gel (EtOAc: heptane $=1: 2)$ yielded the product $(60.8 \mathrm{mg}, 70 \%)$ as a pale yellow solid.

${ }^{1} \mathrm{H}$ NMR $\left(400 \mathrm{MHz}, \mathrm{CDCl}_{3}\right): \delta=8.29(\mathrm{~d}, J=1.6 \mathrm{~Hz}, 1 \mathrm{H}), 7.97-7.89(\mathrm{~m}, 3 \mathrm{H}), 7.69-7.59(\mathrm{~m}, 3 \mathrm{H}), 7.28-7.15(\mathrm{~m}$, 3H), $7.05-7.00(\mathrm{~m}, 2 \mathrm{H}), 4.66(\mathrm{dd}, J=9.0,6.1 \mathrm{~Hz}, 1 \mathrm{H}), 3.76-3.67(\mathrm{~m}, 1 \mathrm{H}), 3.42-3.33(\mathrm{~m}, 1 \mathrm{H}), 3.31-3.20(\mathrm{~m}$, $2 \mathrm{H}), 3.01(\mathrm{~s}, 3 \mathrm{H}), 2.63-2.53(\mathrm{~m}, 1 \mathrm{H}), 2.52-2.42(\mathrm{~m}, 1 \mathrm{H}), 2.17-2.05(\mathrm{~m}, 1 \mathrm{H}), 1.94-1.82(\mathrm{~m}, 2 \mathrm{H}), 1.81-1.70$ (m, 2H), $1.54-1.44(\mathrm{~m}, 1 \mathrm{H}) ;{ }^{13} \mathrm{C}$ NMR (175 MHz, CDCl $): \delta=167.7,140.8,136.2,135.1,132.4,129.6,129.3$ ， 129.0 , 128.7 (2C), 128.7 (3C), $128.2,127.8,126.5,122.7,56.5$, $46.7,46.2$, 32.4 , 30.8 , 29.8 , 26.4 , 24.3; HRMS (ESI): calculated for $[\mathrm{M}+\mathrm{H}]^{+}\left(\mathrm{C}_{25} \mathrm{H}_{29} \mathrm{~N}_{2} \mathrm{O}_{3} \mathrm{~S}\right) \mathrm{m} / \mathrm{z}$ : 437.1893, found 437.1892; IR (neat, $\left.\mathbf{c m}^{-1}\right)$ : 3058, 3025, $2969,2876,1642,1447,1336,1159,933,751,702,651$. 


\section{4k: 2-(1H-Indol-1-yl)-4-phenyl-1-(pyrrolidin-1-yl)butan-1-one}<smiles>O=C(C(CCc1ccccc1)n1ccc2ccccc21)N1CCCC1</smiles>

The product was prepared according to general procedure E. Purification by column chromatography on silica gel (EtOAc: heptane $=1: 2)$ yielded the product $(51.4 \mathrm{mg}, 77 \%)$ as a pale yellow liquid.

${ }^{1} \mathrm{H}$ NMR $\left(400 \mathrm{MHz}, \mathrm{CDCl}_{3}\right): \delta=7.63(\mathrm{~d}, J=7.7 \mathrm{~Hz}, 1 \mathrm{H}), 7.34-7.25(\mathrm{~m}, 3 \mathrm{H}), 7.25-7.17(\mathrm{~m}, 3 \mathrm{H}), 7.17-7.08(\mathrm{~m}$, $3 \mathrm{H}), 6.55(\mathrm{~d}, J=3.3 \mathrm{~Hz}, 1 \mathrm{H}), 4.97-4.92(\mathrm{~m}, 1 \mathrm{H}), 3.58-3.39(\mathrm{~m}, 2 \mathrm{H}), 3.28-3.20(\mathrm{~m}, 1 \mathrm{H}), 2.88-2.79(\mathrm{~m}, 1 \mathrm{H}), 2.65$ - $2.52(\mathrm{~m}, 3 \mathrm{H}), 2.43-2.31(\mathrm{~m}, 1 \mathrm{H}), 1.87-1.66(\mathrm{~m}, 4 \mathrm{H}) ;{ }^{13} \mathrm{C} \mathrm{NMR}\left(175 \mathrm{MHz}, \mathrm{CDCl}_{3}\right): \delta=167.9,140.9,136.1,128.7$, 128.7 (2C), 128.6 (2C), 126.4, 126.3, 121.8, 121.3, 119.9, 109.1, 102.7, 56.3, 46.5, 46.3, 33.8, 32.0, 26.2, 24.1; HRMS (ESI): calculated for [M+Na] ${ }^{+}\left(\mathrm{C}_{22} \mathrm{H}_{24} \mathrm{~N}_{2} \mathrm{ONa}\right) \mathrm{m} / \mathrm{z}$ : 355.1781, found 355.1780; IR (neat, $\left.\mathbf{c m}^{-1}\right)$ : 3026, 2969, $2875,1645,1456,1400,1308,1192,700$.

\section{I: Methyl 8-((N,4-dimethylphenyl)sulfonamido)-9-oxo-9-(pyrrolidin-1-yl)nonanoate}

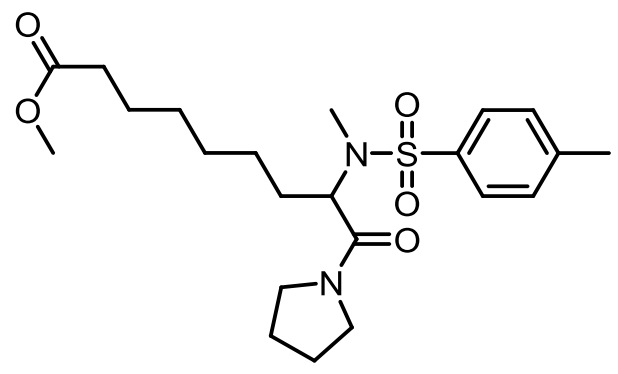

The product was prepared according to general procedure E. Purification by column chromatography on silica gel (EtOAc: heptane $=1: 1)$ yielded the product $(55.8 \mathrm{mg}, 64 \%)$ as a pale yellow liquid.

${ }^{1} \mathrm{H}$ NMR $\left(400 \mathrm{MHz}, \mathrm{CDCl}_{3}\right): \delta=7.63(\mathrm{~d}, J=8.3 \mathrm{~Hz}, 2 \mathrm{H}), 7.28(\mathrm{~d}, J=8.3 \mathrm{~Hz}, 2 \mathrm{H}), 4.64-4.58(\mathrm{~m}, 1 \mathrm{H}), 3.86-3.76(\mathrm{~m}$, $1 \mathrm{H}), 3.67(\mathrm{~s}, 3 \mathrm{H}), 3.50-3.31(\mathrm{~m}, 2 \mathrm{H}), 3.30-3.24(\mathrm{~m}, 1 \mathrm{H}), 2.91(\mathrm{~s}, 3 \mathrm{H}), 2.42(\mathrm{~s}, 3 \mathrm{H}), 2.28(\mathrm{t}, J=7.6 \mathrm{~Hz}, 2 \mathrm{H}), 2.01-$ $1.91(\mathrm{~m}, 2 \mathrm{H}), 1.88-1.79(\mathrm{~m}, 2 \mathrm{H}), 1.79-1.69(\mathrm{~m}, 1 \mathrm{H}), 1.63-1.52(\mathrm{~m}, 2 \mathrm{H}), 1.33-1.11(\mathrm{~m}, 7 \mathrm{H}) ;{ }^{13} \mathrm{C}$ NMR (175

$\left.\mathrm{MHz}, \mathrm{CDCl}_{3}\right): \delta=174.2,168.3,143.5,136.4,129.6(2 \mathrm{C}), 127.2(2 \mathrm{C}), 56.9,51.5,46.6,45.9,34.1,30.3,28.9$ (2C), $28.2,26.3,26.0,24.9,24.2$, 21.6; HRMS (ESI): calculated for [M+Na] ${ }^{+}\left(\mathrm{C}_{22} \mathrm{H}_{34} \mathrm{~N}_{2} \mathrm{O}_{5} \mathrm{SNa}\right) \mathrm{m} / \mathrm{z}: 461.2081$, found 461.2079; IR (neat, cm cm $^{-1}$ ) 2931, 2860, 1734, 1641, 1437, 1335, 1154, 1088, 815, 713, 652. 


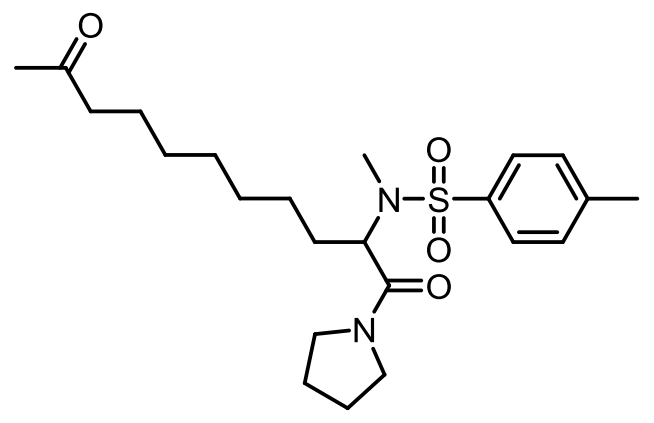

The product was prepared according to general procedure E. Purification by column chromatography on silica gel (EtOAc: heptane $=1: 1)$ yielded the product $(60.0 \mathrm{mg}, 69 \%)$ as a pale yellow liquid.

${ }^{1} \mathrm{H}$ NMR $\left(400 \mathrm{MHz}, \mathrm{CDCl}_{3}\right): \delta=7.63(\mathrm{~d}, J=8.3 \mathrm{~Hz}, 2 \mathrm{H}), 7.28(\mathrm{~d}, J=8.3 \mathrm{~Hz}, 2 \mathrm{H}), 4.62(\mathrm{dd}, J=8.2,6.2 \mathrm{~Hz}, 1 \mathrm{H}), 3.85$ - $3.77(\mathrm{~m}, 1 \mathrm{H}), 3.51-3.24(\mathrm{~m}, 3 \mathrm{H}), 2.91(\mathrm{~s}, 3 \mathrm{H}), 2.42(\mathrm{~s}, 3 \mathrm{H}), 2.40(\mathrm{t}, J=7.4 \mathrm{~Hz}, 2 \mathrm{H}), 2.13(\mathrm{~s}, 3 \mathrm{H}), 2.00-1.92(\mathrm{~m}$, 2H), 1.88-1.79 (m, 2H), $1.79-1.69(\mathrm{~m}, 1 \mathrm{H}), 1.60-1.48(\mathrm{~m}, 2 \mathrm{H}), 1.32-1.12(\mathrm{~m}, 9 \mathrm{H}) ;{ }^{13} \mathrm{C} \mathrm{NMR}\left(175 \mathrm{MHz}, \mathrm{CDCl}_{3}\right)$ : $\delta=209.3$, 168.4 , 143.5 , $136.4,129.7$ (2C) 127.3 (2C), 56.9 , $46.7,46.0$, $43.8,30.4,30.0,29.3,29.2,29.2$ ， $28.3,26.3,26.2,24.3,23.9,21.7$; HRMS (ESI): calculated for $[\mathrm{M}+\mathrm{Na}]^{+}\left(\mathrm{C}_{23} \mathrm{H}_{36} \mathrm{~N}_{2} \mathrm{O}_{4} \mathrm{SNa}\right) \mathrm{m} / \mathrm{z}: 459.2288$, found 459.2289; IR (neat, cm cm: $^{-1}$ 2928, 2855, 1711, 1640, 1598, 1441, 1334, 1154, 1088, 911, 814, 730, 651.

4n: N-(7-Cyano-1-oxo-1-(pyrrolidin-1-yl)hexan-2-yl)-N,4-dimethylbenzenesulfonamide

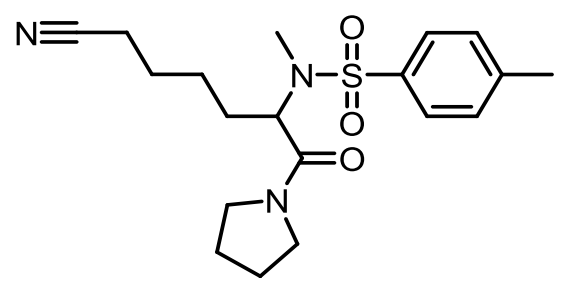

The product was prepared according to general procedure E. Purification by column chromatography on silica gel (EtOAc: heptane $=1: 9-7: 3)$ yielded the product $(32.5 \mathrm{mg}, 47 \%)$ as a pale yellow liquid.

${ }^{1} \mathrm{H}$ NMR $\left(400 \mathrm{MHz}, \mathrm{CDCl}_{3}\right): \delta=7.65(\mathrm{~d}, J=8.2 \mathrm{~Hz}, 2 \mathrm{H}), 7.31(\mathrm{~d}, J=8.2 \mathrm{~Hz}, 2 \mathrm{H}), 4.62(\mathrm{dd}, J=8.9,6.1 \mathrm{~Hz}, 1 \mathrm{H}), 3.85$ $-3.77(\mathrm{~m}, 1 \mathrm{H}), 3.51-3.28(\mathrm{~m}, 3 \mathrm{H}), 2.87(\mathrm{~s}, 3 \mathrm{H}), 2.43(\mathrm{~s}, 3 \mathrm{H}), 2.28(\mathrm{t}, J=7.1 \mathrm{~Hz}, 2 \mathrm{H}), 2.01-1.93(\mathrm{~m}, 2 \mathrm{H}), 1.89-$ $1.76(\mathrm{~m}, 3 \mathrm{H}), 1.72-1.49(\mathrm{~m}, 3 \mathrm{H}), 1.39-1.31(\mathrm{~m}, 2 \mathrm{H}) ;{ }^{13} \mathrm{C} \mathrm{NMR}\left(\mathbf{1 0 0} \mathbf{M H z}, \mathrm{CDCl}_{3}\right): \delta=167.6,143.8,136.3,129.9$ (2C), 127.3 (2C) $119.5,56.9,46.8,46.2$, $30.4,27.3,26.3$, $25.4,25.2$, 24.2 , $21.7,17.1$; HRMS (ESI): calculated for $[\mathrm{M}+\mathrm{Na}]^{+}\left(\mathrm{C}_{19} \mathrm{H}_{27} \mathrm{~N}_{3} \mathrm{O}_{3} \mathrm{SNa}\right) \mathrm{m} / \mathrm{z}: 400.1665$, found 400.1672; IR (neat, $\left.\mathrm{cm}^{-1}\right):$ 2950, 2875, 2246, 1641, 1444, 1334, $1156,1088,816,714,653$. 


\section{4o: N-(6-Chloro-1-oxo-1-(pyrrolidin-1-yl)hexan-2-yl)-N,4-dimethylbenzenesulfonamide}

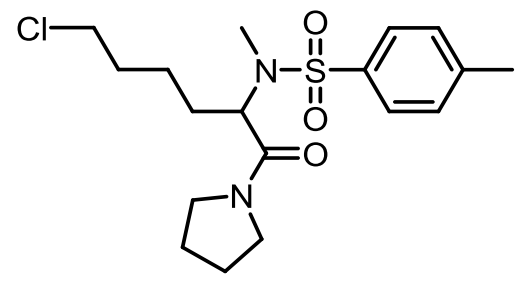

The product was prepared according to general procedure E. Purification by column chromatography on silica gel (EtOAc: heptane $=1: 1)$ yielded the product $(51.2 \mathrm{mg}, 66 \%)$ as a pale yellow liquid.

${ }^{1} \mathrm{H}$ NMR $\left(400 \mathrm{MHz}, \mathrm{CDCl}_{3}\right): \delta=7.65(\mathrm{~d}, J=8.4 \mathrm{~Hz}, 2 \mathrm{H}), 7.30(\mathrm{~d}, J=8.4 \mathrm{~Hz}, 2 \mathrm{H}), 4.63(\mathrm{dd}, J=8.8,6.1 \mathrm{~Hz}, 1 \mathrm{H}), 3.87$ $-3.78(\mathrm{~m}, 1 \mathrm{H}), 3.52-3.27(\mathrm{~m}, 5 \mathrm{H}), 2.89(\mathrm{~s}, 3 \mathrm{H}), 2.42(\mathrm{~s}, 3 \mathrm{H}), 2.02-1.92(\mathrm{~m}, 2 \mathrm{H}), 1.88-1.60(\mathrm{~m}, 5 \mathrm{H}), 1.40-1.21$

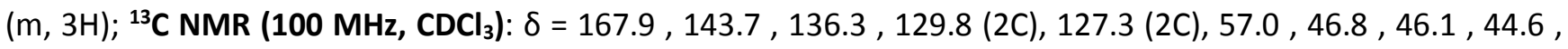
32.2 , $30.4,27.4$, 26.3 , 24.3 , 23.6 , 21.7; HRMS (ESI): calculated for $[\mathrm{M}+\mathrm{Na}]^{+}\left(\mathrm{C}_{18} \mathrm{H}_{27} \mathrm{ClN}_{2} \mathrm{O}_{3} \mathrm{SNa}\right) \mathrm{m} / \mathrm{z}: 409.1323$, found 409.1330; IR (neat, cm $^{-1}$ ): 2951, 2873, 1640, 1441, 1334, 1154, 1088, 929, 912, 815, 713, 652.

\section{4p: N,4-Dimethyl-N-(1-oxo-1-(pyrrolidin-1-yl)undec-10-en-2-yl)benzenesulfonamide}

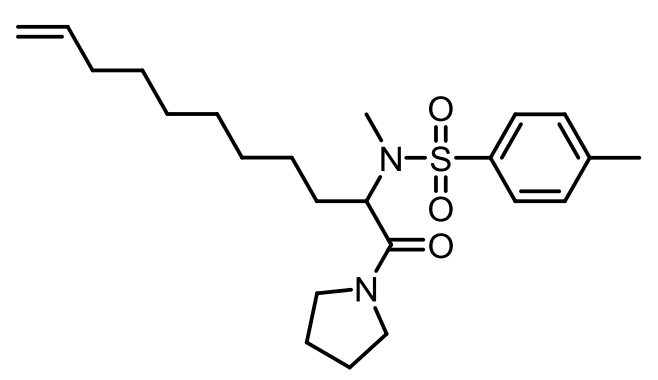

The product was prepared according to general procedure E. Purification by column chromatography on silica gel (EtOAc: heptane $=1: 19-2: 3)$ yielded the product $(39.6 \mathrm{mg}, 47 \%)$ as a pale yellow liquid.

${ }^{1} \mathrm{H}$ NMR $\left(400 \mathrm{MHz}, \mathrm{CDCl}_{3}\right): \delta=7.64(\mathrm{~d}, J=8.3 \mathrm{~Hz}, 2 \mathrm{H}), 7.28(\mathrm{~d}, J=8.3 \mathrm{~Hz}, 2 \mathrm{H}), 5.86-5.75(\mathrm{~m}, 1 \mathrm{H}), 5.03-4.91(\mathrm{~m}$, $2 \mathrm{H}), 4.62(\mathrm{dd}, J=8.0,6.0 \mathrm{~Hz}, 1 \mathrm{H}), 3.86-3.78(\mathrm{~m}, 1 \mathrm{H}), 3.50-3.25(\mathrm{~m}, 3 \mathrm{H}), 2.92(\mathrm{~s}, 3 \mathrm{H}), 2.42(\mathrm{~s}, 3 \mathrm{H}), 2.07-1.92$ (m, 4H), $1.88-1.69(\mathrm{~m}, 3 \mathrm{H}), 1.40-1.12(\mathrm{~m}, 11 \mathrm{H}) ;{ }^{13} \mathrm{C}$ NMR (175 MHz, CDCl $): \delta=168.5,143.5,139.2,136.5$, 129.7 (2C), 127.3 (2C), $114.4,57.0$, $46.7,46.0$, 33.9 , 30.4 , $29.4,29.3,29.1$, 29.0 , 28.3 , 26.3 , 26.2 , 24.3 ， 21.7; HRMS (ESI): calculated for $[\mathrm{M}+\mathrm{Na}]^{+}\left(\mathrm{C}_{23} \mathrm{H}_{36} \mathrm{~N}_{2} \mathrm{O}_{3} \mathrm{SNa}\right) \mathrm{m} / \mathrm{z}: 443.2339$, found 443.2336; IR (neat, $\mathbf{c m}^{-1}$ ): 3070 , 2925, 2855, 1643, 1441, 1338, 1160, 1088, 910, 815, 713, 652. 
4q: N-Methyl-4-nitro-N-(1-oxo-3-phenyl-1-(pyrrolidin-1-yl)propan-2-yl)benzenesulfonamide

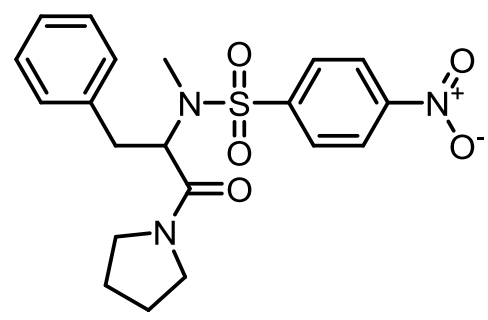

The product was prepared according to general procedure E. Purification by column chromatography on silica gel (EtOAc: heptane $=1: 3)$ yielded the product $(60.0 \mathrm{mg}, 72 \%)$ as a pale yellow solid.

${ }^{1} \mathrm{H}$ NMR $\left(400 \mathrm{MHz}, \mathrm{CDCl}_{3}\right): \delta=8.25(\mathrm{~d}, J=9.0 \mathrm{~Hz}, 2 \mathrm{H}), 7.78(\mathrm{~d}, J=9.0 \mathrm{~Hz}, 2 \mathrm{H}), 7.27-7.21(\mathrm{~m}, 3 \mathrm{H}), 7.14-7.10(\mathrm{~m}$, 2H), $4.94(\mathrm{dd}, J=9.2,6.2 \mathrm{~Hz}, 1 \mathrm{H}), 3.65-3.57(\mathrm{~m}, 1 \mathrm{H}), 3.33-3.12(\mathrm{~m}, 3 \mathrm{H}), 3.13(\mathrm{~s}, 3 \mathrm{H}), 2.87-2.79(\mathrm{~m}, 1 \mathrm{H}), 2.71$ $(\mathrm{dd}, J=13.3,6.2 \mathrm{~Hz}, 1 \mathrm{H}), 1.90-1.64(\mathrm{~m}, 4 \mathrm{H}) ;{ }^{13} \mathrm{C} \mathrm{NMR}\left(100 \mathrm{MHz}, \mathrm{CDCl}_{3}\right): \delta=167.8,150.1,145.0,136.3,129.4$ (2C), 129.0 (2C), 128.5 (2C), $127.4,124.4$ (2C), 58.4 , 46.6 , 45.9 , 36.1 , 31.1 , 26.2 , 24.3; HRMS (ESI): calculated for $[\mathrm{M}+\mathrm{Na}]^{+}\left(\mathrm{C}_{20} \mathrm{H}_{23} \mathrm{~N}_{3} \mathrm{O}_{5} \mathrm{SNa}\right) \mathrm{m} / \mathrm{z}: 440.1244$, found 440.1251; IR (neat, $\mathbf{c m}^{-1}$ ): 3104, 3063, 3029, 2876, 1640, 1528, $1447,1347,1162,942,853,738,699,609$.

4r: N,N-Dimethyl-2-((N-methyl-4-nitrophenyl)sulfonamido)-4-phenylbutanamide

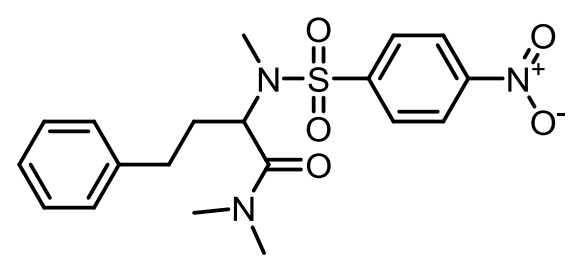

The product was prepared according to general procedure E. Purification by column chromatography on silica gel (EtOAc: heptane $=1: 5-1: 2)$ yielded the product $(60.4 \mathrm{mg}, 74 \%)$ as a pale yellow liquid.

${ }^{1} \mathrm{H}$ NMR $\left(400 \mathrm{MHz}, \mathrm{CDCl}_{3}\right): \delta=8.31(\mathrm{~d}, J=9.0 \mathrm{~Hz}, 2 \mathrm{H}), 7.81(\mathrm{~d}, J=9.0 \mathrm{~Hz}, 2 \mathrm{H}), 7.36-7.30(\mathrm{~m}, 2 \mathrm{H}), 7.28-7.22(\mathrm{~m}$, 1H), $7.16-7.10(\mathrm{~m}, 2 \mathrm{H}), 4.83(\mathrm{apt}, J=7.5 \mathrm{~Hz}, 1 \mathrm{H}), 3.01(\mathrm{~s}, 3 \mathrm{H}), 2.99(\mathrm{~s}, 3 \mathrm{H}), 2.86(\mathrm{~s}, 3 \mathrm{H}), 2.67-2.58(\mathrm{~m}, 1 \mathrm{H}), 2.57$ - $2.48(\mathrm{~m}, 1 \mathrm{H}), 2.17-2.07(\mathrm{~m}, 1 \mathrm{H}), 1.63-1.53(\mathrm{~m}, 1 \mathrm{H}) ;{ }^{13} \mathrm{C} \mathbf{N M R}\left(100 \mathrm{MHz}, \mathrm{CDCl}_{3}\right): \delta=169.3,150.2,144.7$, 140.3 , 128.8 (2C), 128.7 (2C), 128.5 (2C), 126.7 , 124.3 (2C), 54.4 , 37.3 , 36.0 , 32.1 , 30.7 , 30.5; HRMS (ESI): calculated for $[\mathrm{M}+\mathrm{Na}]^{+}\left(\mathrm{C}_{19} \mathrm{H}_{23} \mathrm{~N}_{3} \mathrm{O}_{5} \mathrm{SNa}\right) \mathrm{m} / \mathrm{z}$ : 428.1251, found 428.1248; IR (neat, $\left.\mathbf{c m}^{-1}\right)$ : 3103, 3061, 3026, 2933, $2866,1645,1528,1346,1161,932,854,736,697,606$. 


\section{4s: N,4-dimethyl-N-(1-morpholino-1-oxohexan-2-yl)benzenesulfonamide}<smiles>CCCC(C(=O)N1CCOCC1)N(C)S(=O)(=O)c1ccc(C)cc1</smiles>

The product was prepared according to general procedure E. Purification by column chromatography on silica gel (10-50\% EtOAc in heptane) yielded the product ( $28.4 \mathrm{mg}, 44 \%$ (59\% brsm)) as a pale yellow liquid.

${ }^{1} \mathrm{H}$ NMR (400 MHz, CDCl $): \delta=7.66(\mathrm{~d}, J=8.3 \mathrm{~Hz}, 2 \mathrm{H}), 7.31(\mathrm{~d}, J=8.3 \mathrm{~Hz}, 2 \mathrm{H}), 4.79$ (dd, $\left.J=9.3,5.3 \mathrm{~Hz}, 1 \mathrm{H}\right), 3.89$ $-3.79(\mathrm{~m}, 1 \mathrm{H}), 3.74-3.55(\mathrm{~m}, 6 \mathrm{H}), 3.52-3.40(\mathrm{~m}, 1 \mathrm{H}), 2.83(\mathrm{~s}, 3 \mathrm{H}), 2.43(\mathrm{~s}, 3 \mathrm{H}), 1.98-1.89(\mathrm{~m}, 2 \mathrm{H}), 1.87-1.75$ $(\mathrm{m}, 1 \mathrm{H}), 1.28-1.09(\mathrm{~m}, 3 \mathrm{H}), 0.86(\mathrm{t}, J=7.0 \mathrm{~Hz}, 3 \mathrm{H}) ;{ }^{13} \mathrm{C}$ NMR (100 MHz, CDCl $): \delta=168.3,143.8,136.2,129.8$ (2C), 127.4 (2C), 67.1 (2C), 54.6, 46.4, 42.6, 30.3, 30.1, 21.7, 19.6, 13.8; HRMS (ESI): calculated for [M+Na] $]^{+}$ $\left(\mathrm{C}_{17} \mathrm{H}_{26} \mathrm{~N}_{2} \mathrm{NaO}_{4} \mathrm{~S}\right.$ ) m/z: 377.1505, found 377.1502; IR (neat, $\left.\mathbf{c m}^{-1}\right):=2960,2925,2856,1649,1453,1337,1163$, $1115,934,816$.

\section{5a: $N$-(tert-butoxycarbonyl)-0-(1-oxo-4-phenyl-1-(pyrrolidin-1-yl)butan-2-yl)-L-threonine}<smiles>C[C@@H](OC(CCc1ccccc1)C(=O)N1CCCC1)[C@@H](NC(=O)OC(C)(C)C)C(=O)O</smiles>

The product was prepared according to general procedure $\mathrm{E}$ using $\mathrm{N}$-(tert-Butoxycarbonyl)-L-threonine methyl ester (commercialy available) as a nucleophile. Product Isolated as the free carboxylic Acid. Purification by flash column chromatography (EtOAc: heptane $=1: 2-5: 1$ ) as a yellow oil $(72.0 \mathrm{mg}, 83 \%, 1: 1.1 \mathrm{~d}$.r. determined by crude ${ }^{1} \mathrm{H}$ NMR).

${ }^{1} \mathrm{H}$ NMR (600 MHz, $\mathrm{CDCl}_{3}$ ) Diastereoisomers present : $\delta=7.32-7.29(\mathrm{~m}, 2 \mathrm{H}), 7.19-7.17(\mathrm{~m}, 3 \mathrm{H}), 5.56(\mathrm{~d}, J=$ $8.4 \mathrm{~Hz}, 0.5 \mathrm{H}, \mathrm{d} 1), 5.26(\mathrm{~d}, J=8.4 \mathrm{~Hz}, 0.5 \mathrm{H}, \mathrm{d} 2), 5.06-5.04(\mathrm{~m}, 0.5 \mathrm{H}, \mathrm{d} 1), 4.99-4.98(\mathrm{~m}, 0.5 \mathrm{H}, \mathrm{d} 2), 4.87$ (bs, $0.5 \mathrm{H}$, d1), 4.68 (bs, 0.5H, d2), $4.43-4.42(\mathrm{~m}, 0.5 \mathrm{H}, \mathrm{d} 2), 4.29$ (bs, $0.5 \mathrm{H}), 4.22(\mathrm{~m}, J=6.0 \mathrm{~Hz}, 0.5 \mathrm{H}), 4.09(\mathrm{~d}, J=9.0 \mathrm{~Hz}$, $0.5 \mathrm{H}, \mathrm{d} 1), 3.53-3.47(\mathrm{~m}, 1 \mathrm{H}), 3.41-3.29(\mathrm{~m}, 2 \mathrm{H}), 3.13-3.06(\mathrm{~m}, 1 \mathrm{H}), 2.90-2.84(\mathrm{~m}, 1 \mathrm{H}), 2.74-2.67(\mathrm{~m}, 1 \mathrm{H})$, 
$2.24-2.17(\mathrm{~m}, 1 \mathrm{H}), 2.02-1.76(\mathrm{~m}, 5 \mathrm{H}), 1.47(\mathrm{bs}, 4.5 \mathrm{H}), 1.46(\mathrm{bs}, 4.5 \mathrm{H}), 1.28(\mathrm{~d}, J=6.6 \mathrm{~Hz}, 1.5 \mathrm{H}), 1.25(\mathrm{~d}, J=6.6$ $\mathrm{Hz}, 1.5 \mathrm{H}) .{ }^{13} \mathrm{C}$ NMR (151 MHz, $\left.\mathrm{CDCl}_{3}\right): \delta=172.4$ (d1) , 172.1 (d2) , 169.1 (d1) , 169.0 (d2) , 156.5 (d1) , 156.4 (d2) , 140.4 (d1) , 140.3 (d2) , 128.8 (d1) (2C) , 128.8 (d2) (2C) , 128.7 (d1) (2C) , 128.6 (d2) (2C) , $126.6,80.0$ (d1) , $79.8(\mathrm{~d} 2), 72.3(\mathrm{~d} 1)$, $72.0(\mathrm{~d} 2), 68.5(\mathrm{~d} 1), 68.0(\mathrm{~d} 2), 60.2(\mathrm{~d} 1), 59.4(\mathrm{~d} 2), 46.5,45.9,32.1$ (d1) , 32.0 (d2) , 31.6 (d1) , $31.4(\mathrm{~d} 2), 28.5(\mathrm{~d} 1)(3 \mathrm{C}), 28.5(\mathrm{~d} 2)(3 \mathrm{C}), 26.2$ (d1) , 26.2 (d2) , 23.9 (d1) , 23.9 (d2) , 19.5 (d1) , 18.6 (d2) ; HRMS (ESI): calculated for $[\mathrm{M}+\mathrm{Na}]^{+}\left(\mathrm{C}_{23} \mathrm{H}_{34} \mathrm{~N}_{2} \mathrm{O}_{6} \mathrm{Na}\right) \mathrm{m} / \mathrm{z}: 457.2315$ found [M+Na] ${ }^{+}$: 457.2299; IR (neat, $\left.\mathbf{c m}^{-1}\right)$ : 3359, 2975, 2931, 2882, 1745, 1712, 1635, 1496, 1453.

5b: 2-(((3aR,5R,6S,6aR)-5-((R)-2,2-dimethyl-1,3-dioxolan-4-yl)-2,2-dimethyltetrahydrofuro[2,3-d][1,3]dioxol6-yl)oxy)-4-phenyl-1-(pyrrolidin-1-yl)butan-1-one

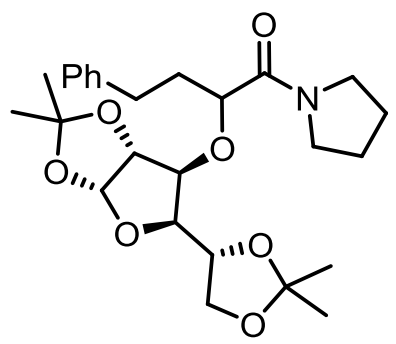

The product was prepared according to general procedure E. Purification by flash column chromatography (EtOAc: heptane $=1: 2-7: 1$ ) as a yellow oil $\left(87.4 \mathrm{mg}, 92 \%\right.$, d.r $1: 1$, determined by crude ${ }^{1} \mathrm{H}$ NMR).

${ }^{1} \mathrm{H}$ NMR $\left(600 \mathrm{MHz}, \mathrm{CDCl}_{3}\right)$ : Diastereoisomers are present $\delta=7.28-7.26(\mathrm{~m}, 2 \mathrm{H}), 7.24-7.23(\mathrm{~m}, 1 \mathrm{H}), 7.20-7.17$ $(\mathrm{m}, 2 \mathrm{H}), 5.77(\mathrm{~d}, J=3.8 \mathrm{~Hz}, 0.5 \mathrm{H}, \mathrm{d} 1), 5.67(\mathrm{~d}, J=3.5 \mathrm{~Hz}, 0.5 \mathrm{H}, \mathrm{d} 2), 4.57-4.54(\mathrm{~m}, 1 \mathrm{H}), 4.40-4.38(\mathrm{~m}, 0.5 \mathrm{H}, \mathrm{d} 1)$, 4.26 - $4.23(\mathrm{~m}, 1.5 \mathrm{H}), 4.18-4.16(\mathrm{~m}, 0.5 \mathrm{H}, \mathrm{d} 2), 4.09-4.07(\mathrm{~m}, 0.5 \mathrm{H}, \mathrm{d} 1), 4.05-3.98(\mathrm{~m}, 2 \mathrm{H}), 3.85$ - $3.79(\mathrm{~m}$, 1.5H), $3.53-3.49(\mathrm{~m}, 0.5 \mathrm{H}, \mathrm{d} 1), 3.47-3.30(\mathrm{~m}, 3 \mathrm{H}), 2.87-2.81(\mathrm{~m}, 1 \mathrm{H}), 2.77-2.74(\mathrm{~m}, 0.5 \mathrm{H}, \mathrm{d} 1), 2.71-2.67$ $(\mathrm{m}, 0.5 \mathrm{H}, \mathrm{d} 2), 2.16-2.05(\mathrm{~m}, 1.5 \mathrm{H}), 1.99-1.96(\mathrm{~m}, 0.5 \mathrm{H}, \mathrm{d} 1), 1.91-1.85(\mathrm{~m}, 2 \mathrm{H}), 1.83-1.78(\mathrm{~m}, 2 \mathrm{H}), 1.59$ (bs, 1.5H, d1), 1.55 (bs, 1.5H, d2), 1.45 (bs, 1.5H, d1), 1.44 (bs, 1.5H, d2), 1.35 (bs, 4.5H), 1.31(bs, 1.5H, d1); ${ }^{13}$ C NMR (151 MHz, $\mathrm{CDCl}_{3}$ ): $\delta$ = 169.9 (d1) , 169.5 (d2), 141.8 (d1) , 141.5 (d2) , 128.7 , 128.6 , $128.5,128.5,128.4,126.2$ (d1) , 126.1 (d2) , 113.0 (d1) , 113.0 (d2) , 109.8 (d1) , 109.6 (d2) , 104.5 (d1) , 103.7 (d2) , 82.0 , $80.3,78.9,78.4$ , 78.1 , 77.6 , 76.7 , 76.5 , 75.5 , 75.4 , 65.7 , 65.0 , 46.7 , 46.3 , 45.9 , 45.9 , 33.6 , 33.3 , 31.9 , 31.6 , 27.1 , 27.1 , $27.0,27.0,26.7,26.5,26.5,26.4,26.4,25.6,25.2,23.9,23.8$; HRMS (ESI): calculated for [M+Na] ${ }^{+}$ $\left(\mathrm{C}_{26} \mathrm{H}_{37} \mathrm{NO}_{7} \mathrm{Na}\right) \mathrm{m} / \mathrm{z}: 498.2468$ found [M+Na] $]^{+}$: 498.2460; IR (neat, $\mathbf{c m}^{-1}$ ): 2982, 2933, 2875, 1635, 1631, 1452. 
<smiles>COC(=O)[C@H](CSC(CCc1ccccc1)C(=O)N1CCCC1)NC(=O)OC(C)(C)C</smiles>

The product was prepared according to general procedure E. Purification by flash column chromatography (EtOAc: heptane $=1: 1-4: 1$ ) as a yellow oil (78.3mg, 87\%, d.r. 1:1, determined by crude ${ }^{1} \mathrm{H} N M R$ ).

${ }^{1} \mathrm{H}$ NMR $\left(600 \mathrm{MHz}, \mathrm{CDCl}_{3}\right): \delta=7.29-7.25(\mathrm{~m}, 2 \mathrm{H}), 7.20-7.15(\mathrm{~m}, 3 \mathrm{H}), 5.33(\mathrm{bs}, 1 \mathrm{H}), 4.57$ (bs, 0.5H, d1), 4.39 (bs, $0.5 \mathrm{H}, \mathrm{d} 2), 3.73(\mathrm{~s}, 1.5 \mathrm{H}, \mathrm{d} 1), 3.72(\mathrm{~s}, 1.5 \mathrm{H}, \mathrm{d} 2), 3.64-3.58(\mathrm{~m}, 3 \mathrm{H}), 3.20-3.17(\mathrm{~m}, 1 \mathrm{H}), 2.77-2.63(\mathrm{~m}, 2 \mathrm{H}), 2.39$ - $2.33(\mathrm{~m}, 1 \mathrm{H}), 2.04-2.00(\mathrm{~m}, 1 \mathrm{H}), 1.99-2.65(\mathrm{~m}, 4 \mathrm{H}), 1.45(\mathrm{~s}, 4.5 \mathrm{H}, \mathrm{d} 1), 1.43(\mathrm{~s}, 4.5 \mathrm{H}, \mathrm{d} 2) ;{ }^{13} \mathrm{C}$ NMR (151 MHz, $\mathrm{CDCl}_{3}$ ): $\delta=171.5$, 169.0 (d1) , 168.7 (d2) , 155.3 (d1) , 155.2 (d2) , 140.9 (d1) , 140.9 (d2) , 128.5 (C2), 128.4 (C2), $126.1,80.0,53.7$ (d1) , 53.3 (d2) , 52.5 , 46.2 , 46.2 , 46.1 , 46.0 , 33.2 , $33.1,33.0,30.9$ (d1) , 30.3 (d2) , 28.3 , 26.0 , 24.3 (d1) , 24.3 (d2) , 24.4 (d2); HRMS (ESI): calculated for [M+Na] ${ }^{+}\left(\mathrm{C}_{23} \mathrm{H}_{34} \mathrm{~N}_{2} \mathrm{O}_{5} \mathrm{SNa}\right) \mathrm{m} / \mathrm{z}: 473.2086$ found $[\mathrm{M}+\mathrm{Na}]^{+}:$473.2080; IR (neat, cm $\mathrm{cm}^{-1}$ ): 3289, 2973, 2929, 2875, 1745, 1711, 1628, 1517, 1435.

\section{5d: Methyl N-(1-oxo-4-phenyl-1-(pyrrolidin-1-yl)butan-2-yl)-N-tosylglycinate}

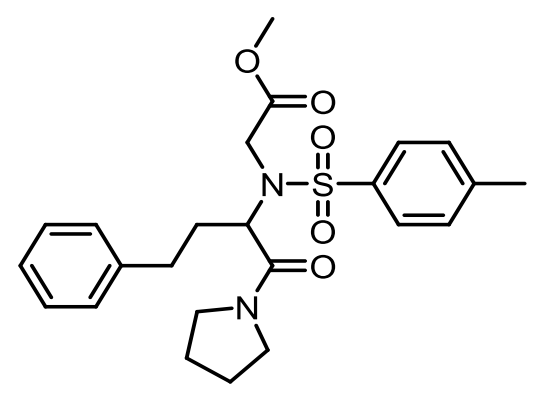

The product was prepared according to general procedure E. Purification by column chromatography on silica gel (EtOAc: heptane $=1: 2)$ yielded the product $(58.0 \mathrm{mg}, 63 \%)$ as a pale yellow liquid.

${ }^{1} \mathrm{H}$ NMR $\left(400 \mathrm{MHz}, \mathrm{CDCl}_{3}\right): \delta=7.66(\mathrm{~d}, J=8.3 \mathrm{~Hz}, 2 \mathrm{H}), 7.32-7.21(\mathrm{~m}, 4 \mathrm{H}), 7.21-7.16(\mathrm{~m}, 1 \mathrm{H}), 7.04(\mathrm{~d}, J=8.3 \mathrm{~Hz}$, 2H), $4.39(\mathrm{~d}, J=18.3 \mathrm{~Hz}, 1 \mathrm{H}), 4.39-4.34(\mathrm{~m}, 1 \mathrm{H}), 4.17(\mathrm{~d}, J=18.3 \mathrm{~Hz}, 1 \mathrm{H}), 3.71(\mathrm{~s}, 3 \mathrm{H}), 3.54-3.45(\mathrm{~m}, 1 \mathrm{H}), 3.38$ $-3.22(\mathrm{~m}, 2 \mathrm{H}), 3.02-2.93(\mathrm{~m}, 1 \mathrm{H}), 2.66-2.57(\mathrm{~m}, 1 \mathrm{H}), 2.53-2.44(\mathrm{~m}, 1 \mathrm{H}), 2.43(\mathrm{~s}, 3 \mathrm{H}), 2.12-2.01(\mathrm{~m}, 1 \mathrm{H}), 1.90$

$-1.74(\mathrm{~m}, 4 \mathrm{H}), 1.62-1.51(\mathrm{~m}, 1 \mathrm{H}) ;{ }^{13} \mathrm{C}$ NMR $\left(100 \mathrm{MHz}, \mathrm{CDCl}_{3}\right): \delta=170.5,167.4,144.0,140.7,137.0,129.8$ (2C), $128.6(2 \mathrm{C}), 128.6$ (2C), 127.8 (2C), 126.4 , 55.7 , 52.3 , 46.2 , 46.1 , 45.3 , 31.9 , 30.9 , 26.2 , 24.2 , 21.7; HRMS 
(ESI): calculated for $[\mathrm{M}+\mathrm{Na}]^{+}\left(\mathrm{C}_{24} \mathrm{H}_{30} \mathrm{~N}_{2} \mathrm{O}_{5} \mathrm{SNa}\right) \mathrm{m} / \mathrm{z}$ : 481.1768, found 481.1758; IR (neat , $\left.\mathbf{c m}^{-1}\right):$ 2951, 2876, 2251, $1756,1638,1598,1447,1341,1206,1156,1093,909,813,727,700,654$.

\section{6: 2-Amino-4-phenyl-1-(pyrrolidin-1-yl)butan-1-one}<smiles>NC(CCc1ccccc1)C(=O)N1CCCC1</smiles>

To $32 \mathrm{mg}(0.077 \mathrm{mmol}) 4 \mathrm{~h}$ in $0.5 \mathrm{~mL} \mathrm{MeCN} 25.3 \mathrm{mg}(0.23 \mathrm{mmol})$ thiophenol and $42.4 \mathrm{mg}(0.31 \mathrm{mmol})$ freshly ground $\mathrm{K}_{2} \mathrm{CO}_{3}$ was added. The mixture was heated to $50{ }^{\circ} \mathrm{C}$ for $40 \mathrm{~h}$. Then, the solvent was removed under reduced pressure and the crude product was purified by column chromatography on silica gel (EtOAc: $\left.\left(99 \% \mathrm{MeOH}, 1 \% \mathrm{NH}_{4} \mathrm{OH}\right)=9: 1\right)$ to yield amine $6(16.6 \mathrm{mg}, 93 \%)$ as a pale yellow liquid.

${ }^{1} \mathrm{H}$ NMR $\left(400 \mathrm{MHz}, \mathrm{CDCl}_{3}\right): \delta=7.31-7.25(\mathrm{~m}, 2 \mathrm{H}), 7.23-7.16(\mathrm{~m}, 3 \mathrm{H}), 3.54-3.37(\mathrm{~m}, 3 \mathrm{H}), 3.34-3.26(\mathrm{~m}, 1 \mathrm{H})$, $3.19-3.10(\mathrm{~m}, 1 \mathrm{H}), 2.86-2.67(\mathrm{~m}, 2 \mathrm{H}), 1.96-1.74(\mathrm{~m}, 6 \mathrm{H}), 1.71(\mathrm{br}, 2 \mathrm{H}) ;{ }^{13} \mathrm{C}$ NMR $\left(100 \mathrm{MHz}, \mathrm{CDCl}_{3}\right): \delta=174.1$ , $141.7,128.7$ (2C), 128.6 (2C), $126.1,52.4,46.1,46.0$, 36.9 , 32.2 , 26.2 , 24.2; HRMS (ESI): calculated for $[\mathrm{M}+\mathrm{H}]^{+}\left(\mathrm{C}_{14} \mathrm{H}_{21} \mathrm{~N}_{2} \mathrm{O}\right) \mathrm{m} / \mathrm{z}$ : 233.1648, found 233.1647; IR (neat, $\left.\mathbf{~ c m}^{-1}\right)$ : 3363, 3060, 3025, 2950, 2873, 1631, 1449, $1370,1342,752,701$.

7a: 2-(Methylamino)-2-(4-nitrophenyl)-4-phenyl-1-(pyrrolidin-1-yl)butan-1-one

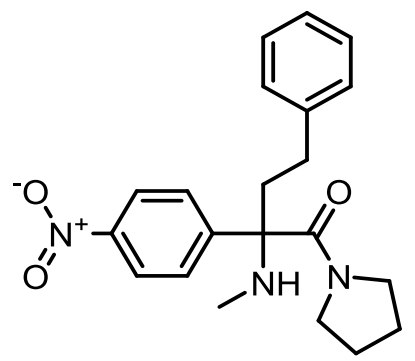

The product was prepared according to general procedure $F$ from amide $4 \mathrm{f}$ ( $63 \mathrm{mg}, 0.15 \mathrm{mmol}$ ). Purification by column chromatography on silica gel (EtOAc: heptane $=1: 1)$ yielded the product $(24.0 \mathrm{mg}, 45 \%)$ as a pale yellow liquid.

${ }^{1} \mathrm{H}$ NMR $\left(400 \mathrm{MHz}, \mathrm{CDCl}_{3}\right): \delta=8.21(\mathrm{~d}, J=9.0 \mathrm{~Hz}, 2 \mathrm{H}), 7.57(\mathrm{~d}, J=9.0 \mathrm{~Hz}, 2 \mathrm{H}), 7.25-7.20(\mathrm{~m}, 2 \mathrm{H}), 7.19-7.13(\mathrm{~m}$, 1H), $7.12-7.06(\mathrm{~m}, 2 \mathrm{H}), 3.53$ (bs, 2H), 3.20 (bs, 1H), 2.70 (bs, 1H), $2.62-2.53(\mathrm{~m}, 1 \mathrm{H}), 2.42-2.33(\mathrm{~m}, 2 \mathrm{H}), 2.28$ (s, 3H), $2.17-1.88(\mathrm{br}, 1 \mathrm{H}) 1.74-1.49(\mathrm{~m}, 5 \mathrm{H}) ;{ }^{13} \mathrm{C}$ NMR (150 MHz, CDCl 3 ): $\delta=170.6,150.0,147.0,141.7$, $128.6(2 \mathrm{C}), 128.4(2 \mathrm{C}), 127.3(2 \mathrm{C}), 126.1,123.8(2 \mathrm{C}), 68.5,47.9,46.6,35.4,29.4,29.2$, 26.8 , 23.2 ; HRMS 
(ESI): calculated for $[\mathrm{M}+\mathrm{H}]^{+}\left(\mathrm{C}_{21} \mathrm{H}_{26} \mathrm{~N}_{3} \mathrm{O}_{3}\right) \mathrm{m} / \mathrm{z}: 368.1969$, found 368.1971; IR (neat, $\mathbf{c m}^{-1}$ ): 2946, 2876, 2798, 1625, $1603,1519,1401,1346,1109,854$.

7b: 2-(Methylamino)-2-(4-nitrophenyl)-3-phenyl-1-(pyrrolidin-1-yl)propan-1-one

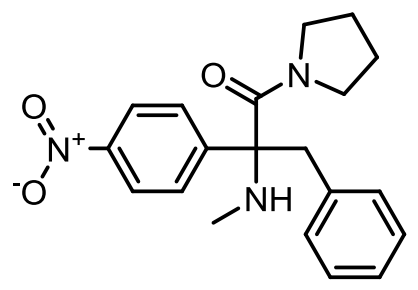

The product was prepared according to general procedure $F$ from amide $4 \mathrm{r}(417.5 \mathrm{mg}, 1 \mathrm{mmol})$. Purification by column chromatography on silica gel (EtOAc: heptane $=3: 2$ ) yielded the product $(159.0 \mathrm{mg}, 45 \%)$ as a pale yellow solid.

${ }^{1} \mathrm{H}$ NMR $\left(600 \mathrm{MHz}, \mathrm{CDCl}_{3}\right): \delta=8.10(\mathrm{~d}, J=9.0 \mathrm{~Hz}, 2 \mathrm{H}), 7.31-7.24(\mathrm{~m}, 2 \mathrm{H}), 7.14-7.11(\mathrm{~m}, 1 \mathrm{H}), 7.11-7.06(\mathrm{~m}$, 2H), $6.52(\mathrm{~d}, J=9.0 \mathrm{~Hz}, 2 \mathrm{H}), 3.71(\mathrm{~d}, J=14.3 \mathrm{~Hz}, 1 \mathrm{H}), 3.67-3.62(\mathrm{~m}, 1 \mathrm{H}), 3.60-3.49(\mathrm{~m}, 2 \mathrm{H}), 3.24(\mathrm{~d}, J=14.3 \mathrm{~Hz}$, 1H), $2.50(\mathrm{~s}, 3 \mathrm{H}), 2.44-2.36(\mathrm{~m}, 1 \mathrm{H}), 1.79-1.62(\mathrm{~m}, 3 \mathrm{H}), 1.61-1.52(\mathrm{~m}, 2 \mathrm{H}) ;{ }^{13} \mathrm{C}$ NMR $\left(150 \mathrm{MHz}, \mathrm{CDCl}_{3}\right): \delta=$ $169.8,149.8,146.7,135.9$, $130.4(2 \mathrm{C}), 128.1$ (2C), 127.2 (2C), $126.8,123.3$ (2C), $69.5,47.6,46.4,41.1,30.1$ , 26.7 , 23.3; HRMS (ESI): calculated for $[\mathrm{M}+\mathrm{H}]^{+}\left(\mathrm{C}_{20} \mathrm{H}_{24} \mathrm{~N}_{3} \mathrm{O}_{3}\right) \mathrm{m} / \mathrm{z}$ : 354.1812, found 354.1816; IR (neat, $\left.\mathbf{c m}^{-1}\right)$ : $3028,2947,2875,2802,1629,1599,1518,1451,1398,1345,1107,850,705$.

\section{7c: N,N-Dimethyl-2-(methylamino)-2-(4-nitrophenyl)-4-phenylbutanamide}

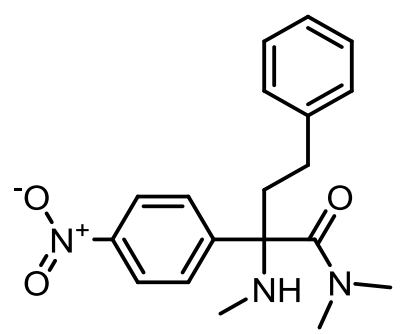

The product was prepared according to general procedure $\mathrm{F}$ from amide $\mathbf{4 q}$ ( $39 \mathrm{mg}, 0.096 \mathrm{mmol})$. Purification by column chromatography on silica gel (EtOAc: heptane $=1: 2)$ yielded the product $(17.0 \mathrm{mg}, 51 \%)$ as a colourless liquid.

${ }^{1} \mathrm{H}$ NMR $\left(400 \mathrm{MHz}, \mathrm{CDCl}_{3}\right): \delta=8.23(\mathrm{~d}, J=9.0 \mathrm{~Hz}, 2 \mathrm{H}), 7.53(\mathrm{~d}, J=9.0 \mathrm{~Hz}, 2 \mathrm{H}), 7.26-7.20(\mathrm{~m}, 2 \mathrm{H}), 7.19-7.13(\mathrm{~m}$, 1H), 7.10-7.04 (m, 2H), 2.91 (bs, 3H), 2.74 (bs, 3H), $2.65-2.53(\mathrm{~m}, 1 \mathrm{H}), 2.39-2.26(\mathrm{~m}, 2 \mathrm{H}), 2.30(\mathrm{~s}, 3 \mathrm{H}), 2.25-$ $2.12(\mathrm{~m}, 1 \mathrm{H}), 1.97$ (bs, 1H); ${ }^{13} \mathrm{C}$ NMR (100 MHz, CDCl $): \delta=171.7,150.4,146.9,141.6,128.6(2 \mathrm{C}), 128.4$ (2C), $126.8(2 \mathrm{C}), 126.1,123.9(2 \mathrm{C}), 68.5,37.6,36.5,29.5$ (2C), 29.4; HRMS (ESI): calculated for $[\mathrm{M}+\mathrm{H}]^{+}\left(\mathrm{C}_{19} \mathrm{H}_{24} \mathrm{~N}_{3} \mathrm{O}_{3}\right)$ 
m/z: 342.1813, found 342.1813. IR (neat, $\mathbf{c m}^{-1}$ ): 2928, 2857, 1666, 1634, 1601, 1518, 1385, 1345, 1256, 1091, 855, 701, 659. 


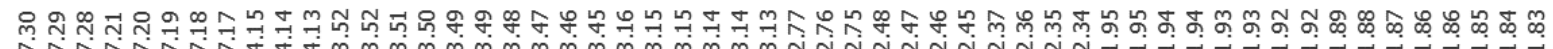

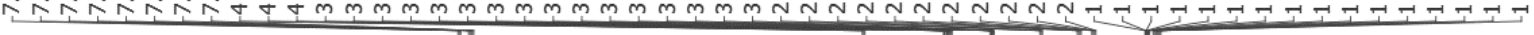<smiles>C#CC#CCCCN1CCCC1</smiles>

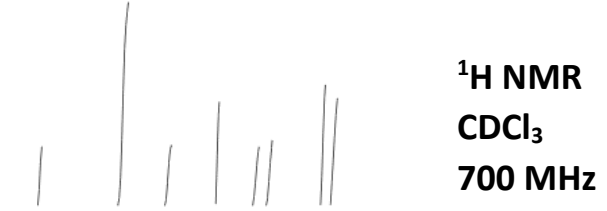

1 aa
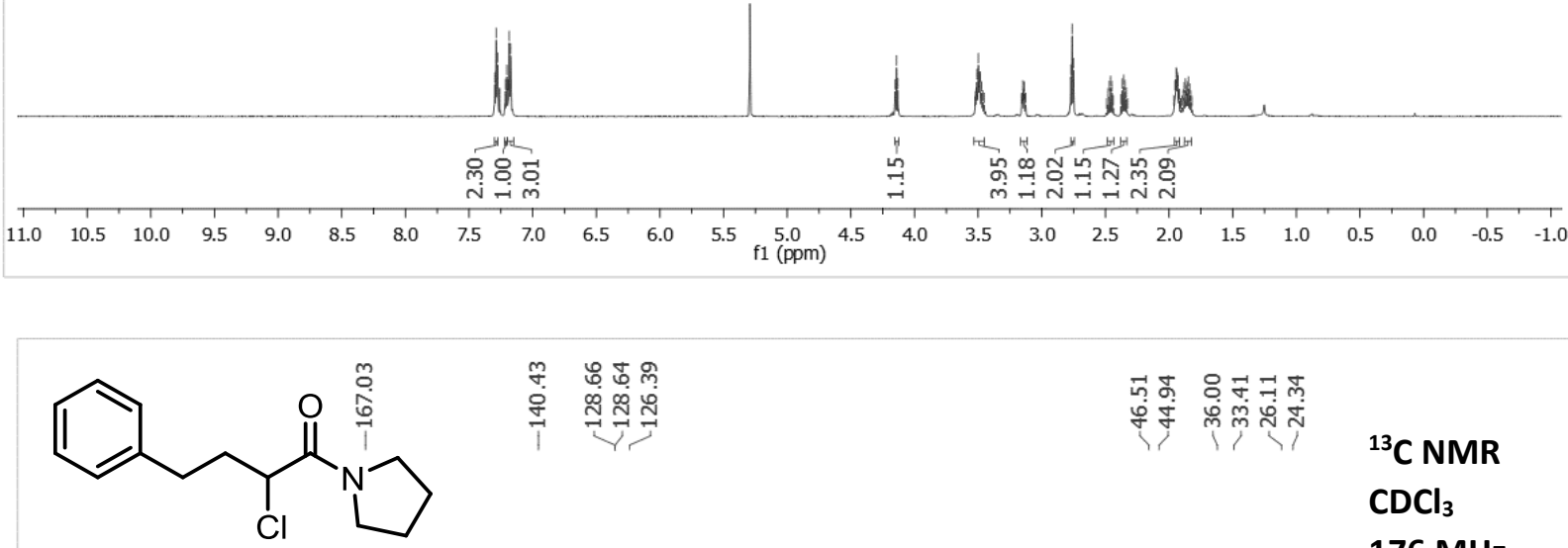

${ }^{13}$ C NMR $\mathrm{CDCl}_{3}$ $176 \mathrm{MHz}$

1 aa

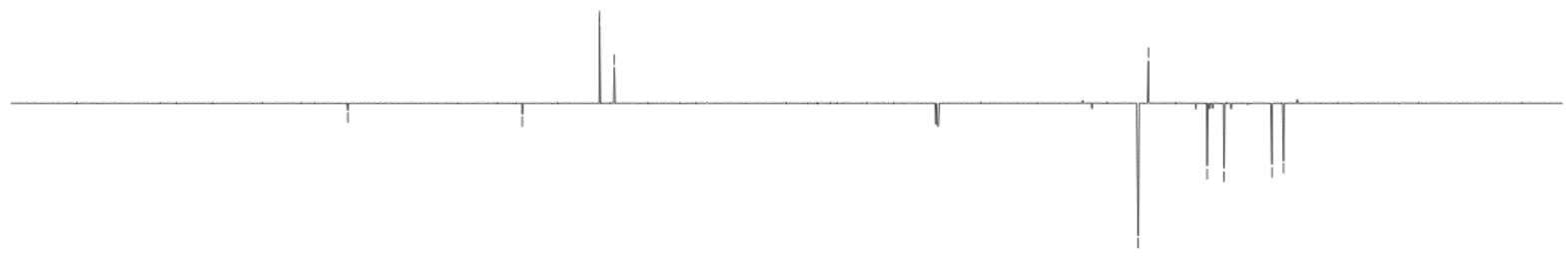

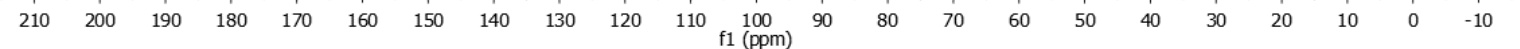




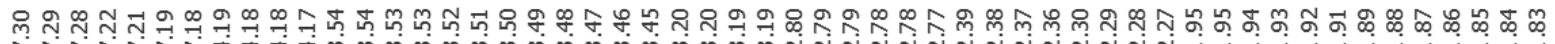

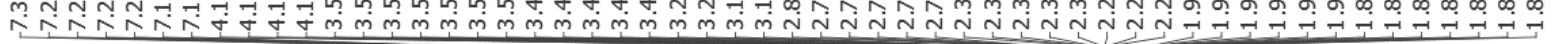<smiles>C#CC1CCCN1C(=O)C(Br)CCc1ccccc1</smiles>

${ }^{1} \mathrm{H}$ NMR

$\mathrm{CDCl}_{3}$ $400 \mathrm{MHz}$

$1 a b$
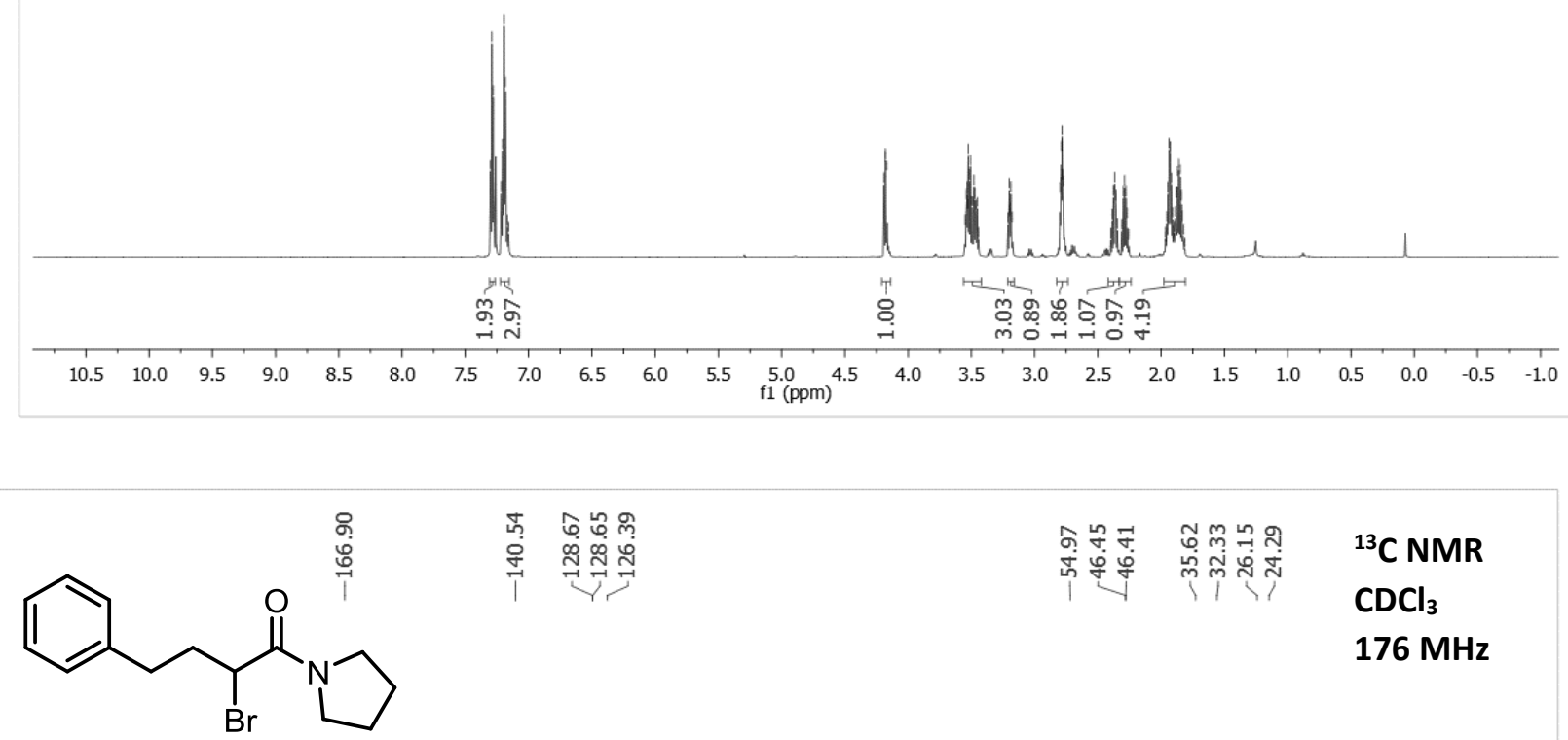

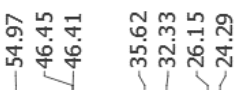

${ }^{13} \mathrm{C}$ NMR

$\mathrm{CDCl}_{3}$

$176 \mathrm{MHz}$

$1 \mathrm{ab}$

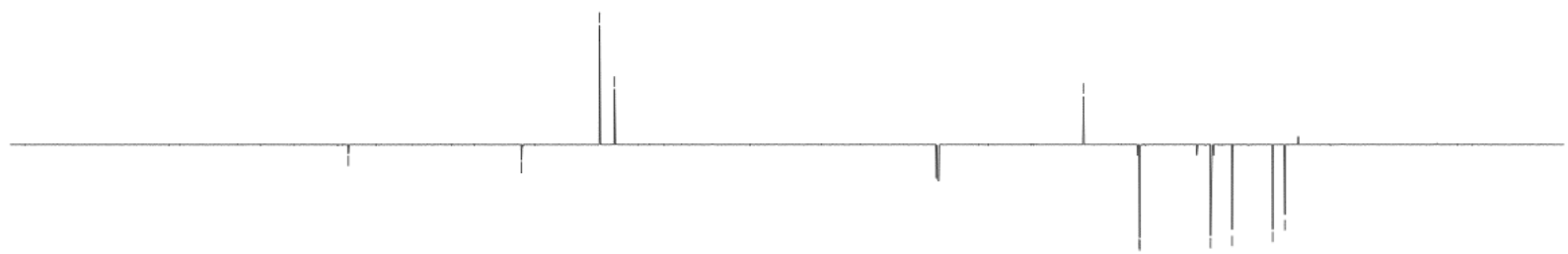

$\begin{array}{lllllllllllllllllllllll}210 & 200 & 190 & 180 & 170 & 160 & 150 & 140 & 130 & 120 & 110 & 100 & 90 & 80 & 70 & 60 & 50 & 40 & 30 & 20 & 10 & 0 & -10\end{array}$ 

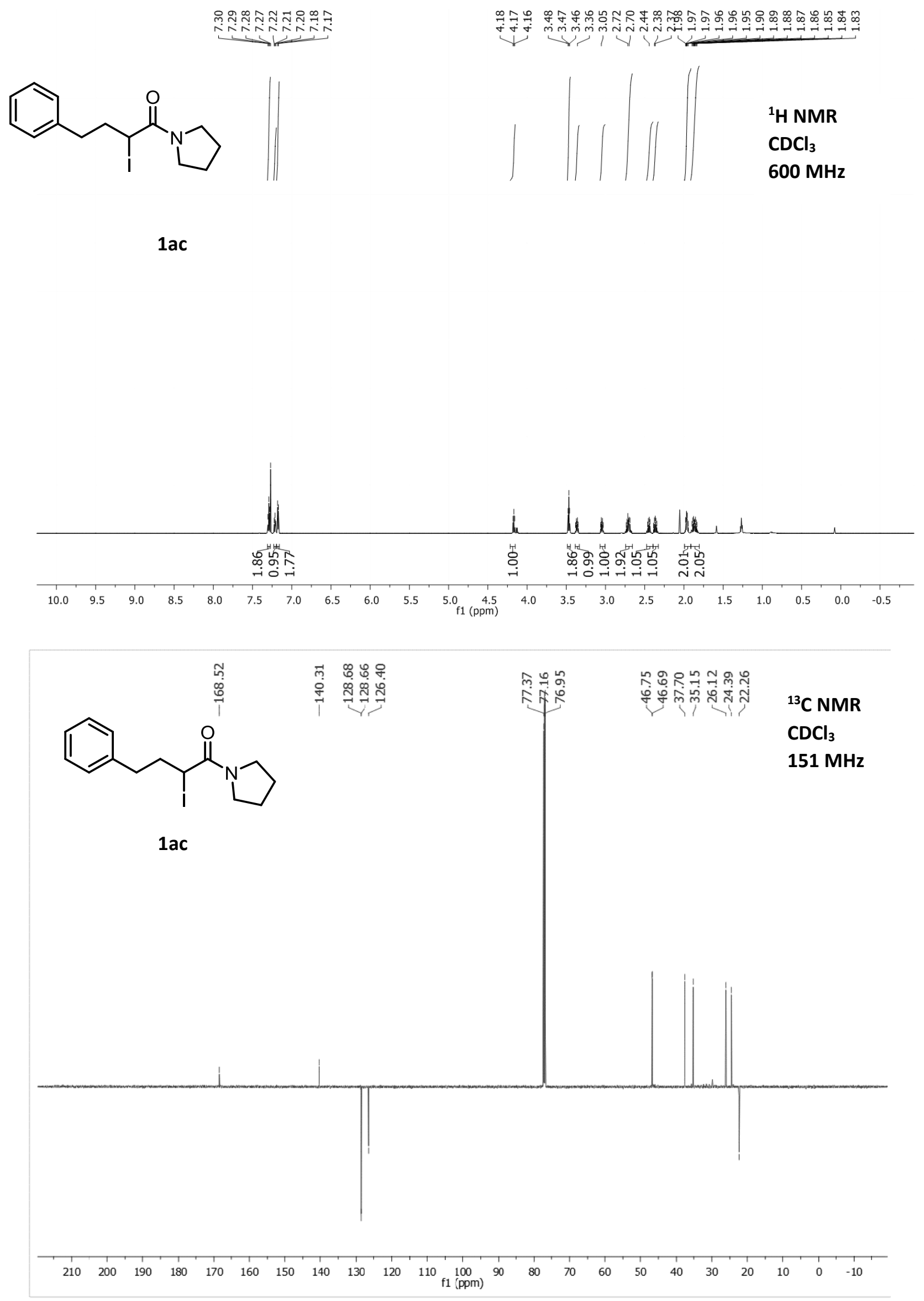


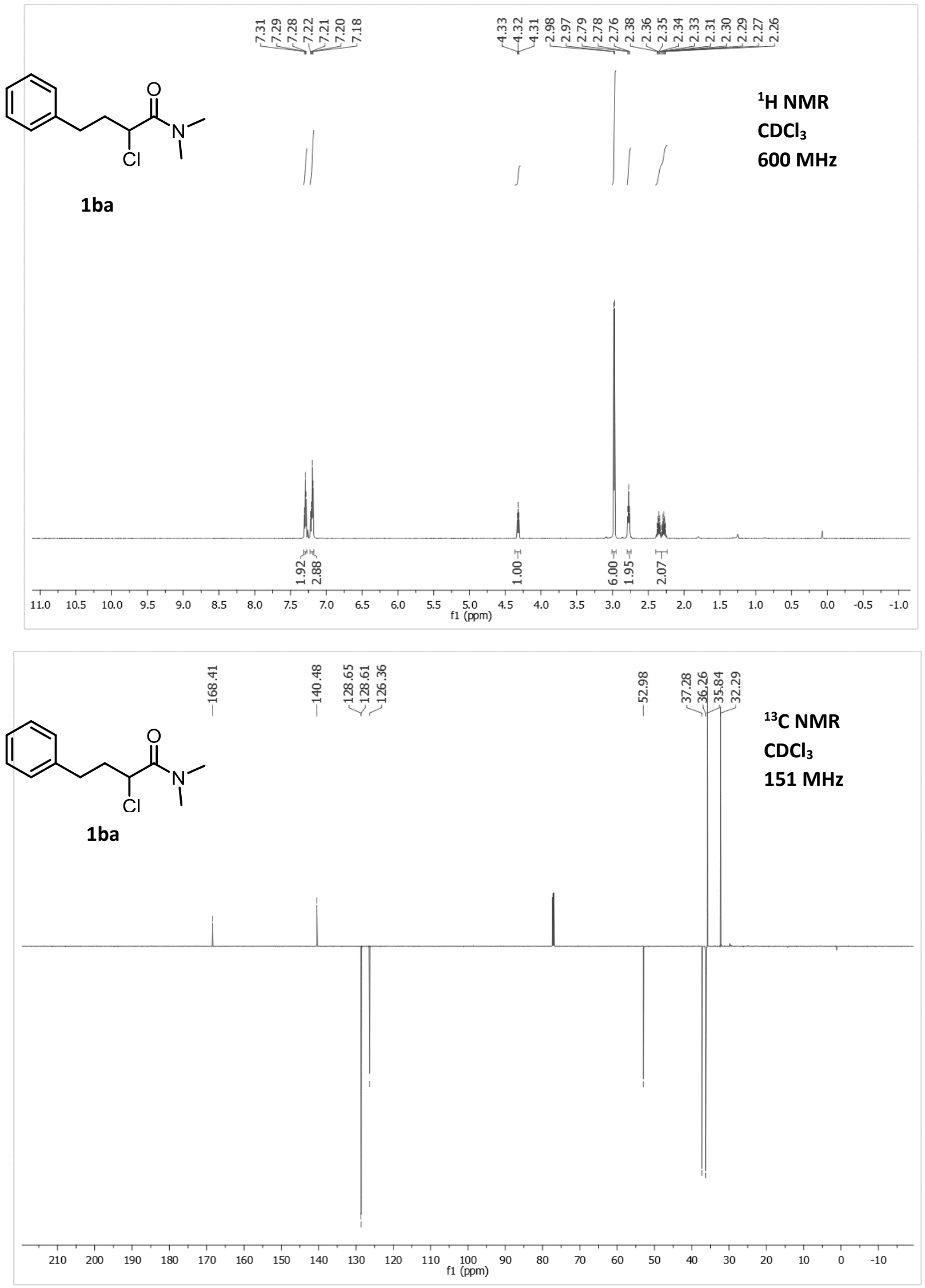




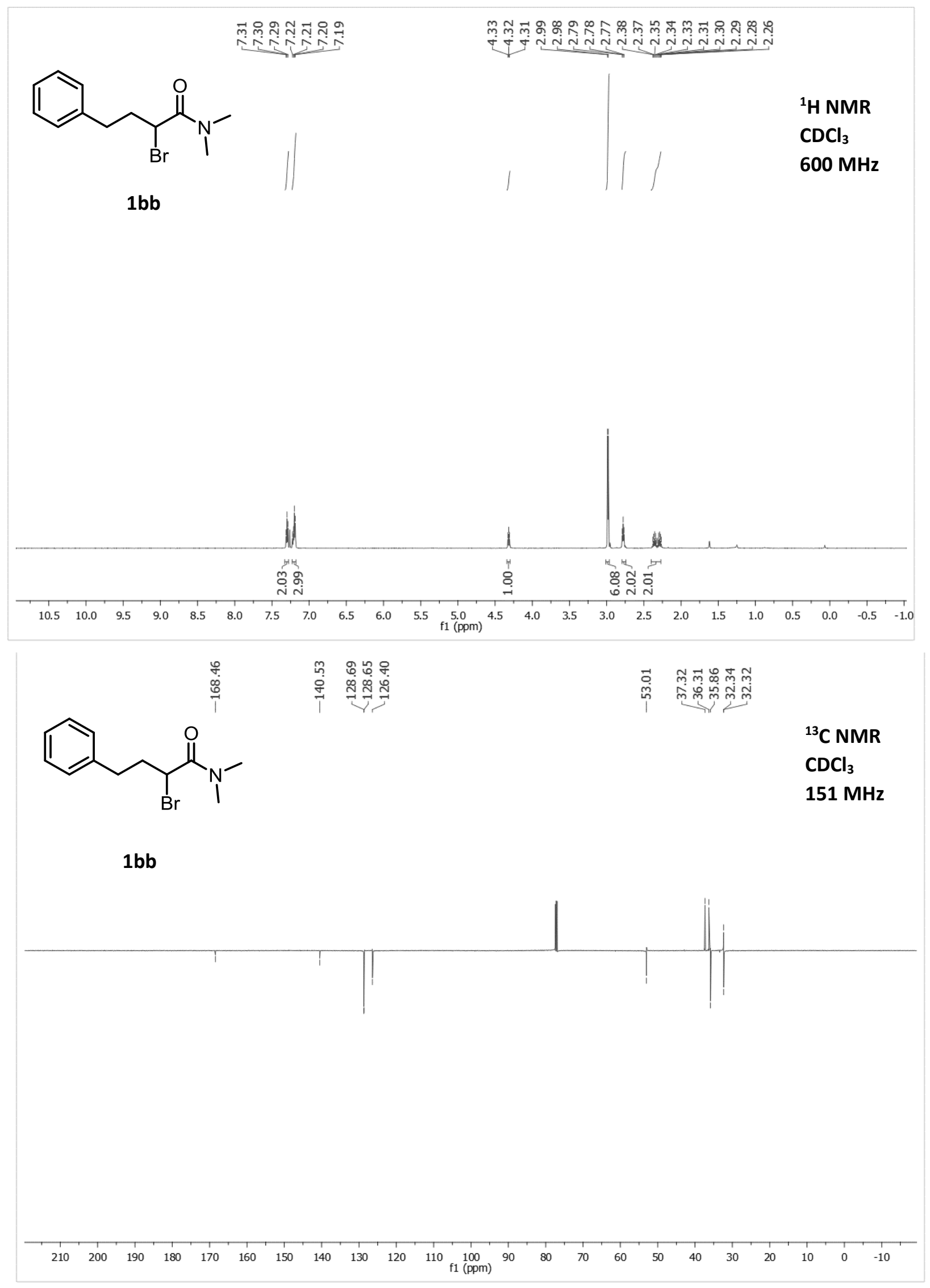



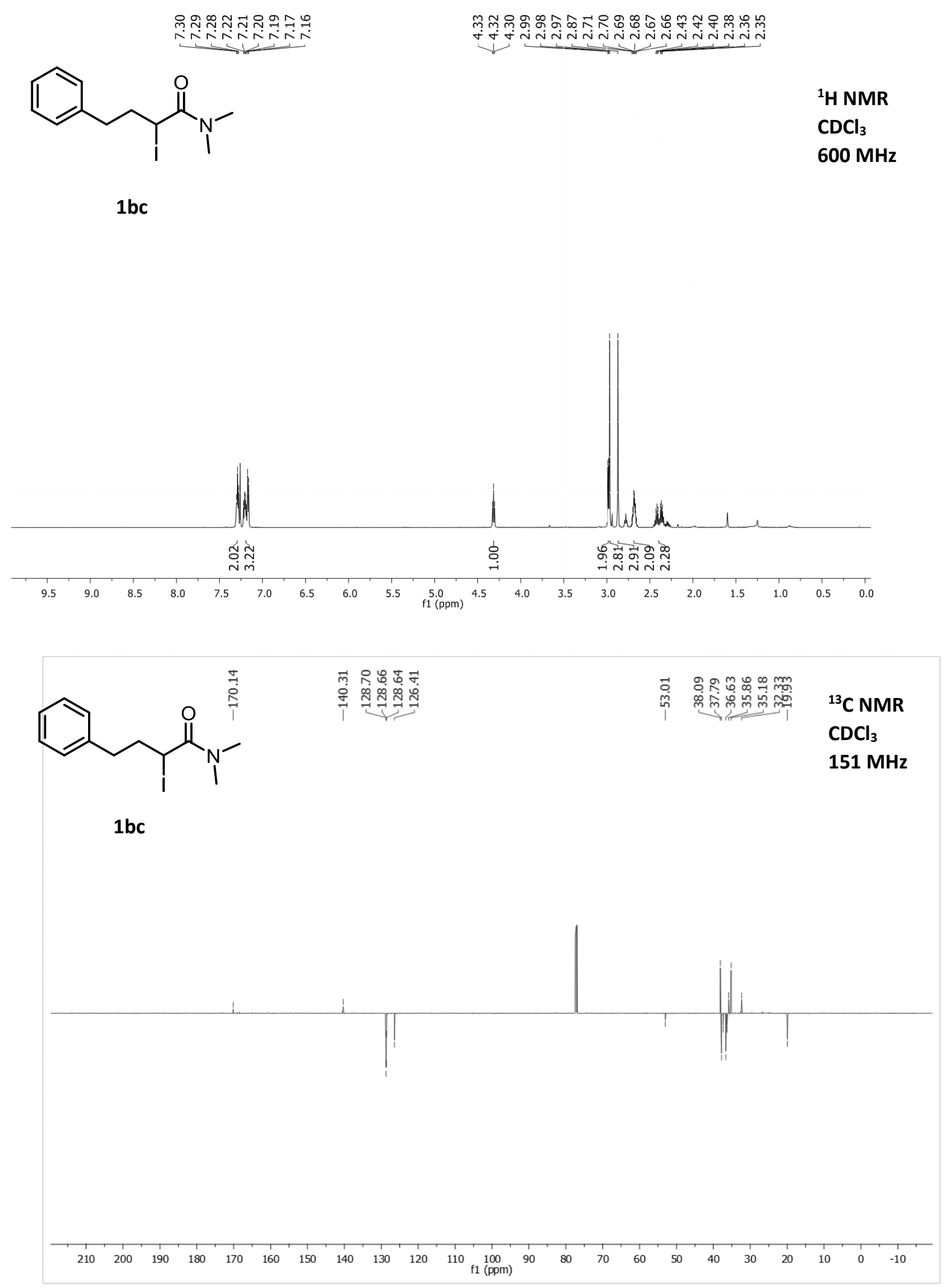

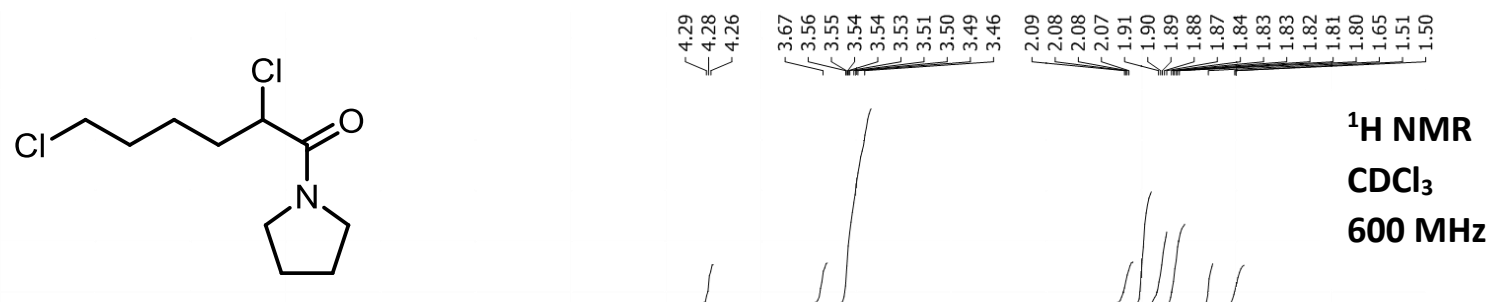

$1 c a$
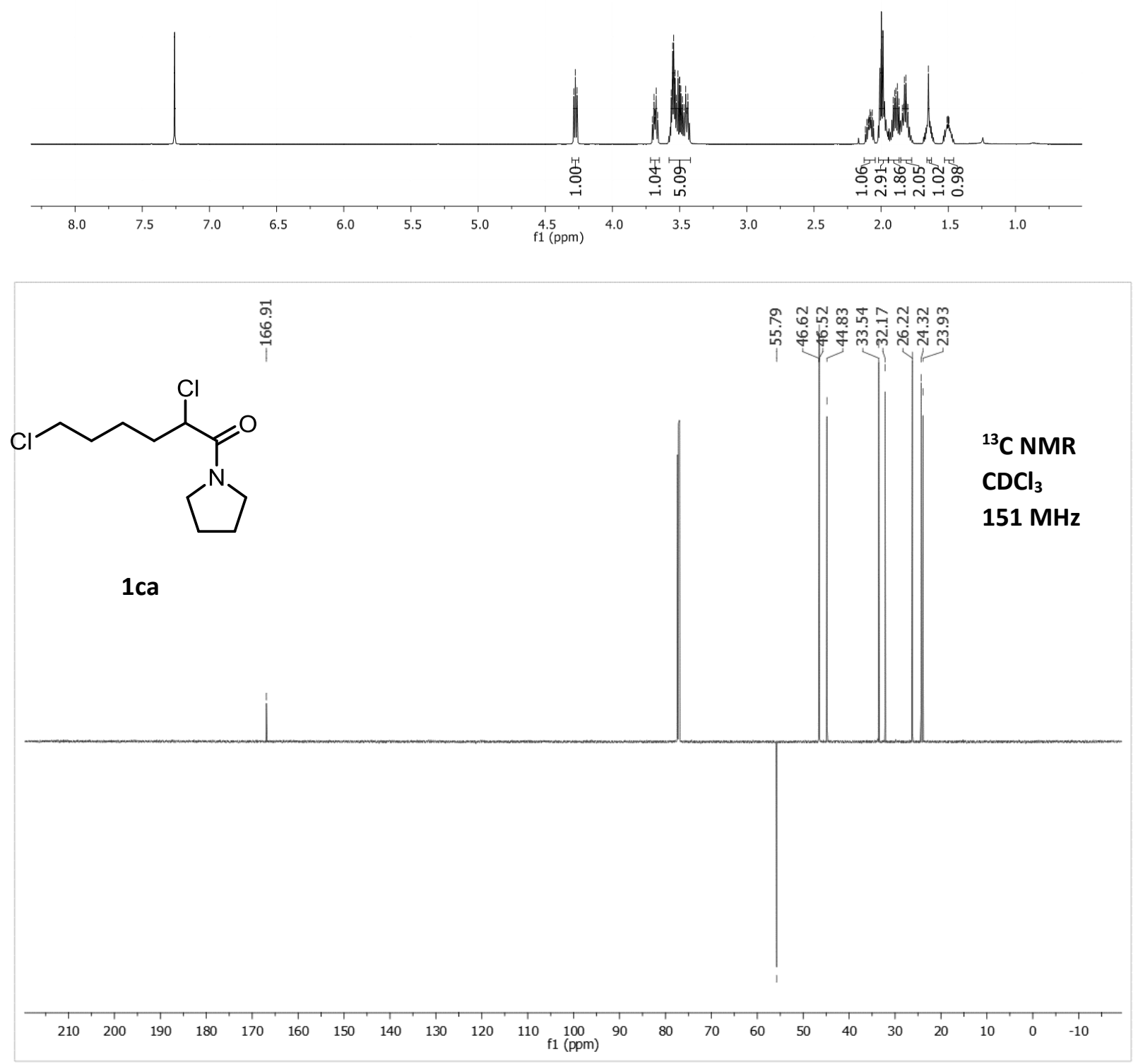


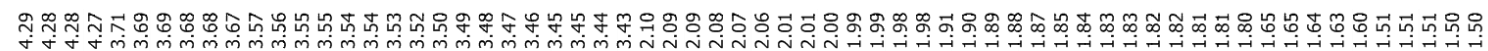<smiles>O=C(C(Br)CCCCCl)N1CCCC1</smiles>

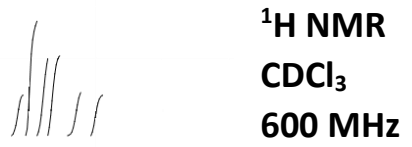

$1 c b$
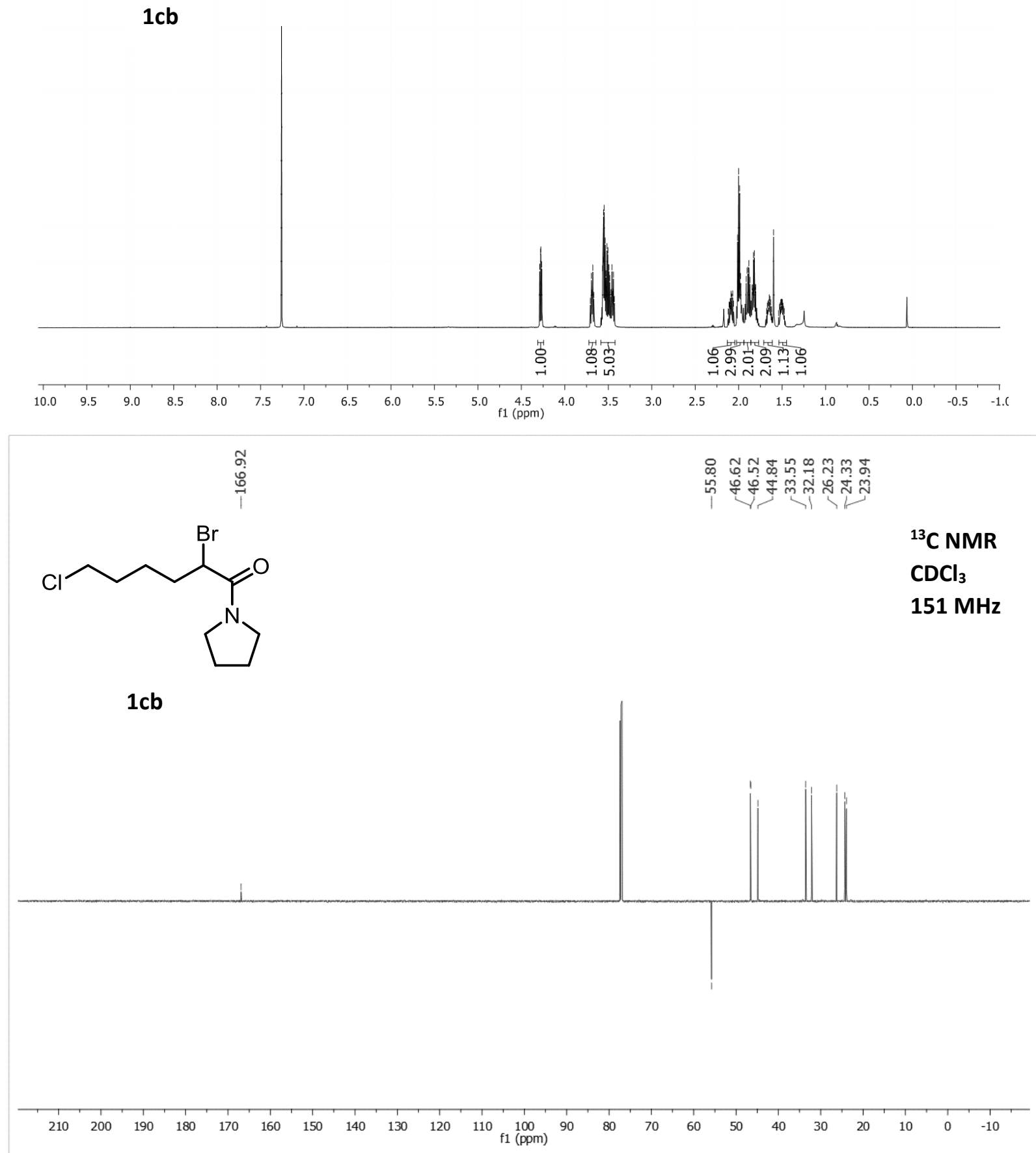


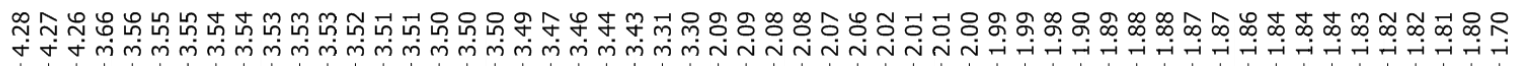

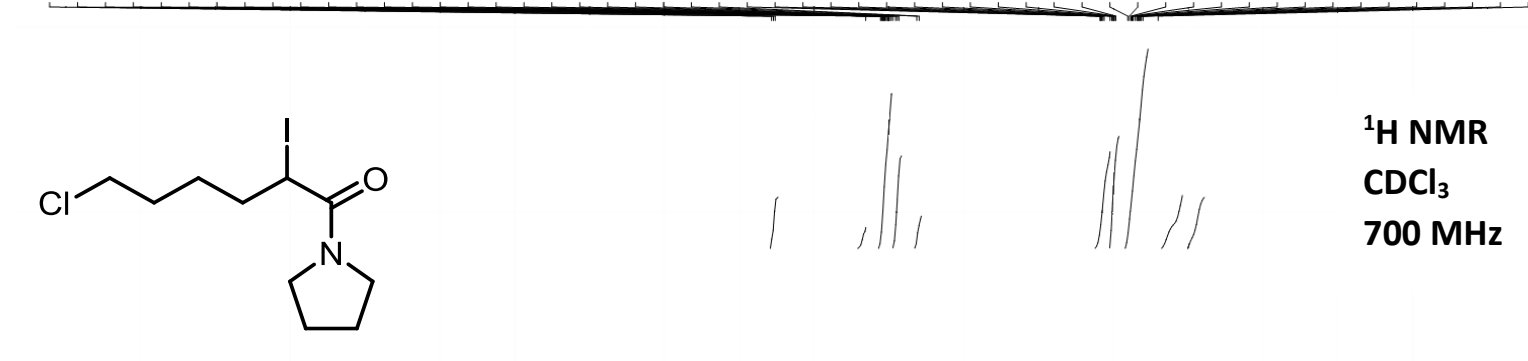

$1 c c$
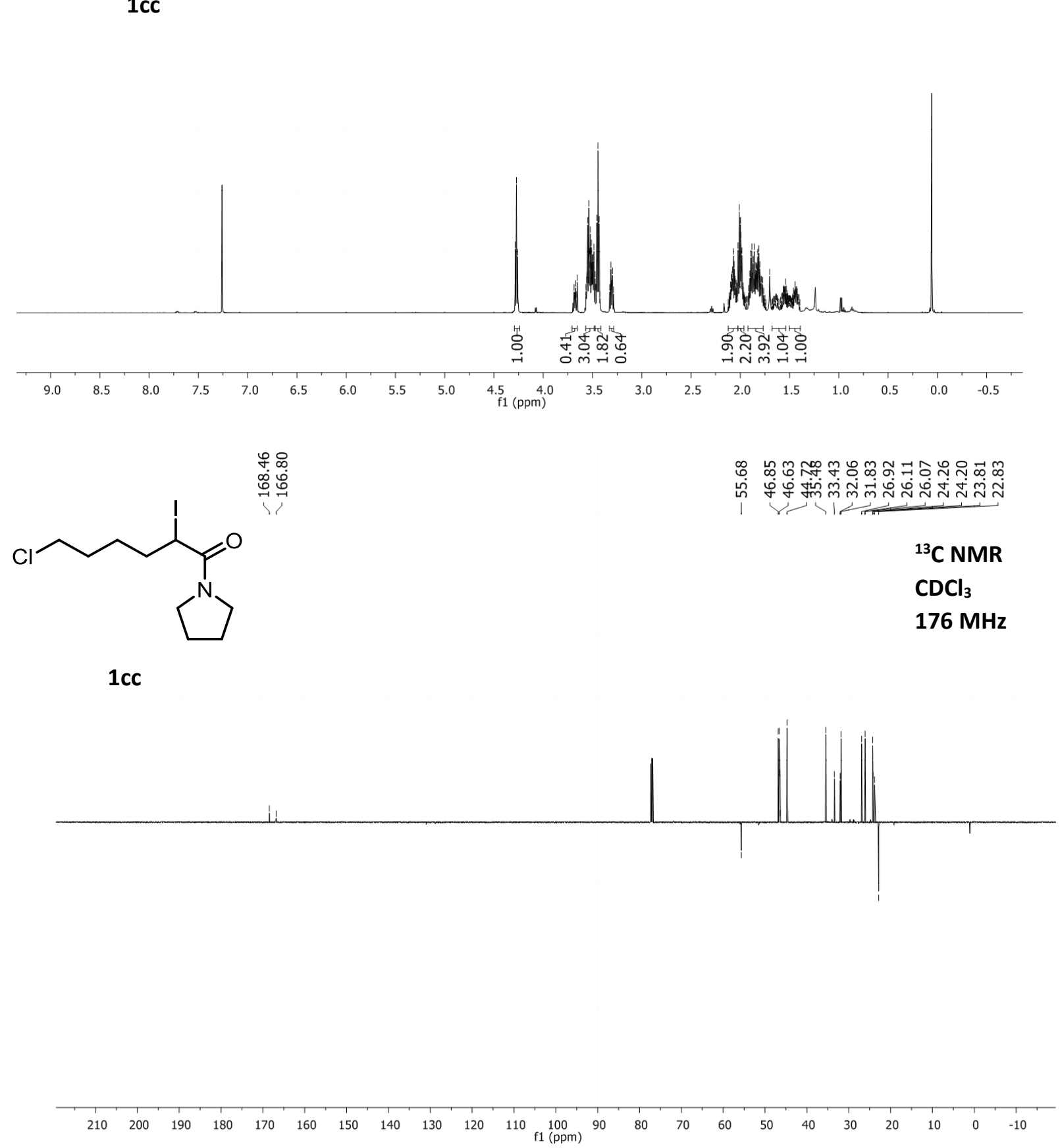

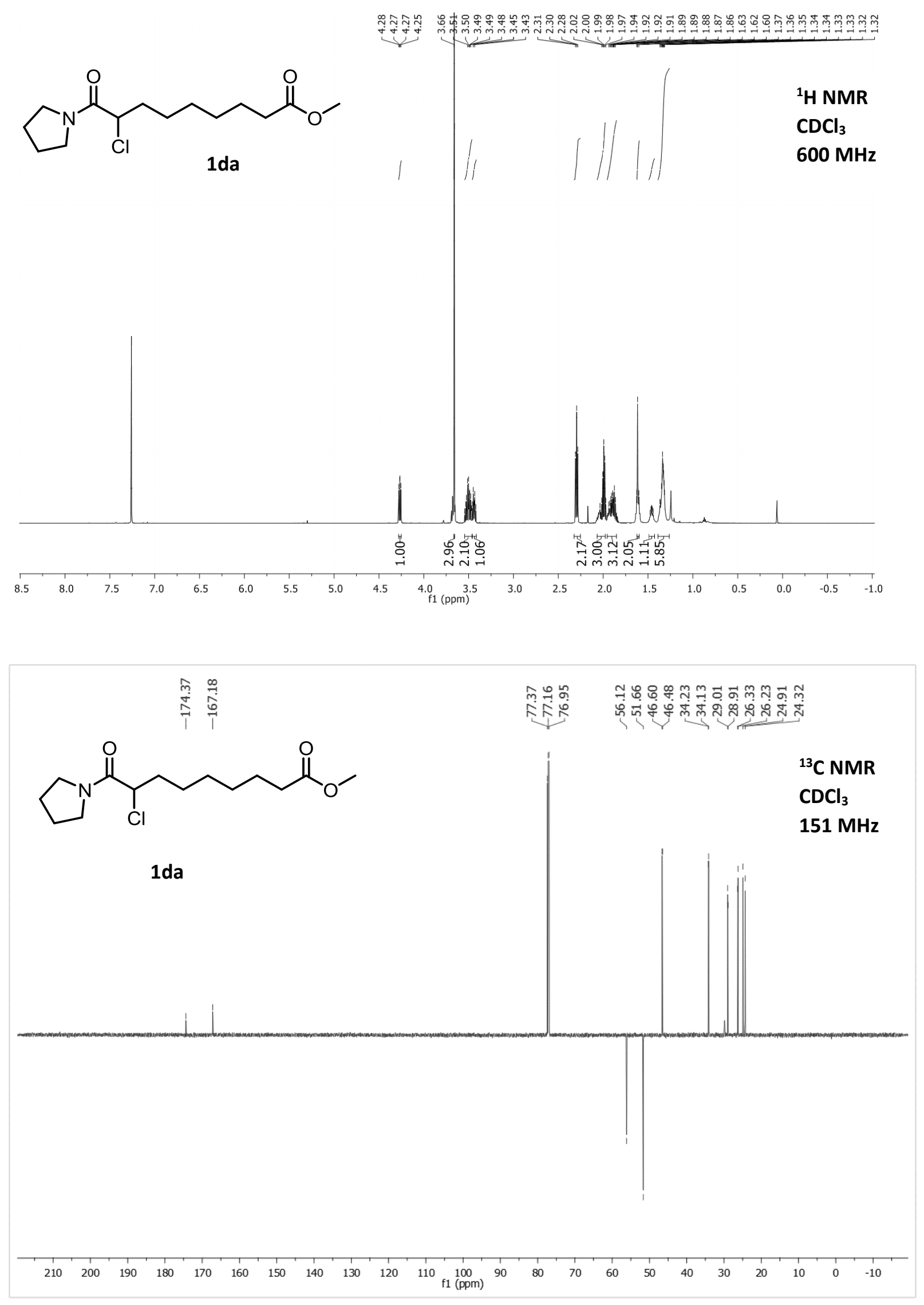

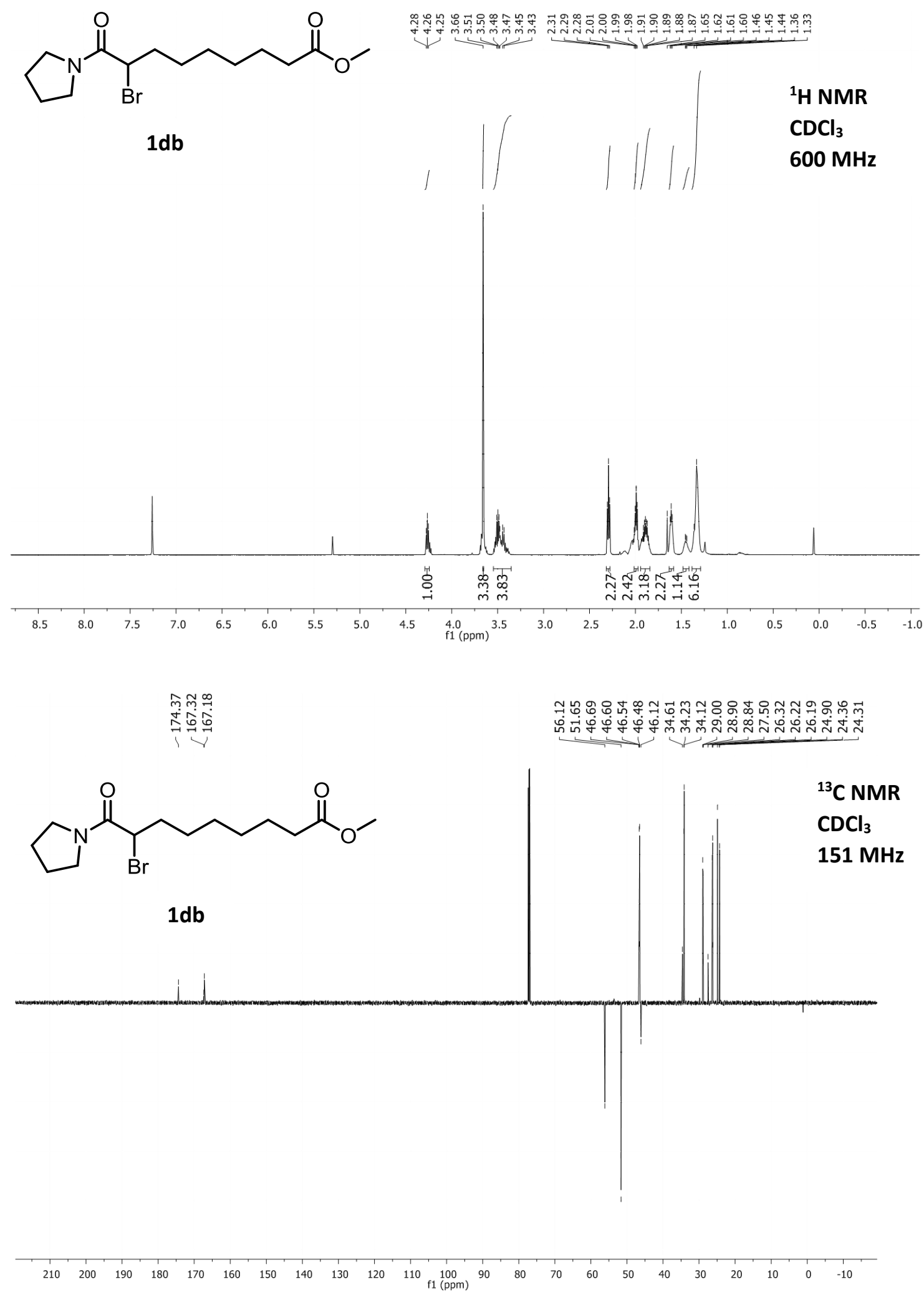

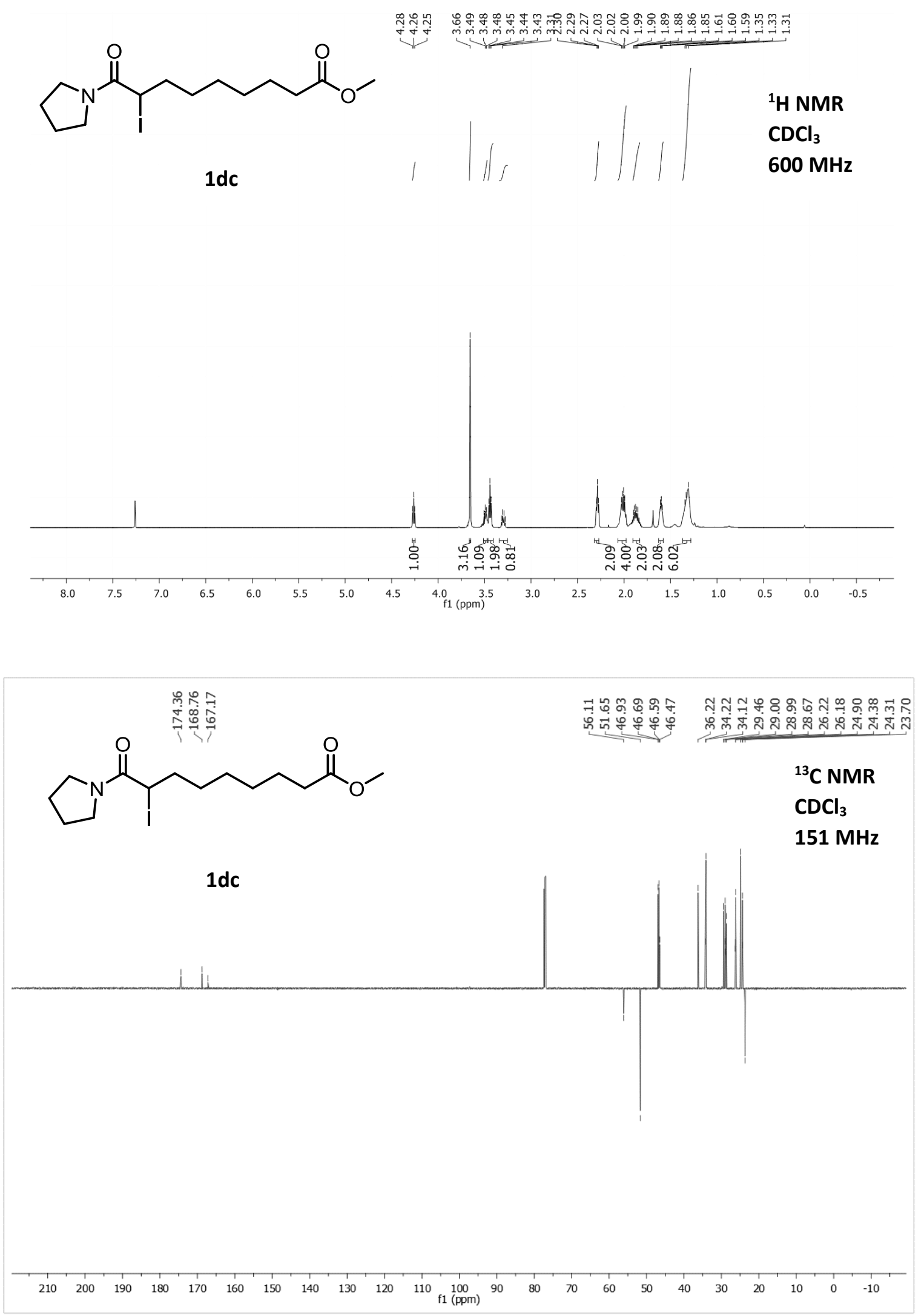


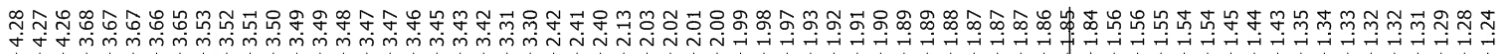<smiles>CC(=O)CCCCCCCC(I)C(=O)N1CCCC1</smiles>

1e
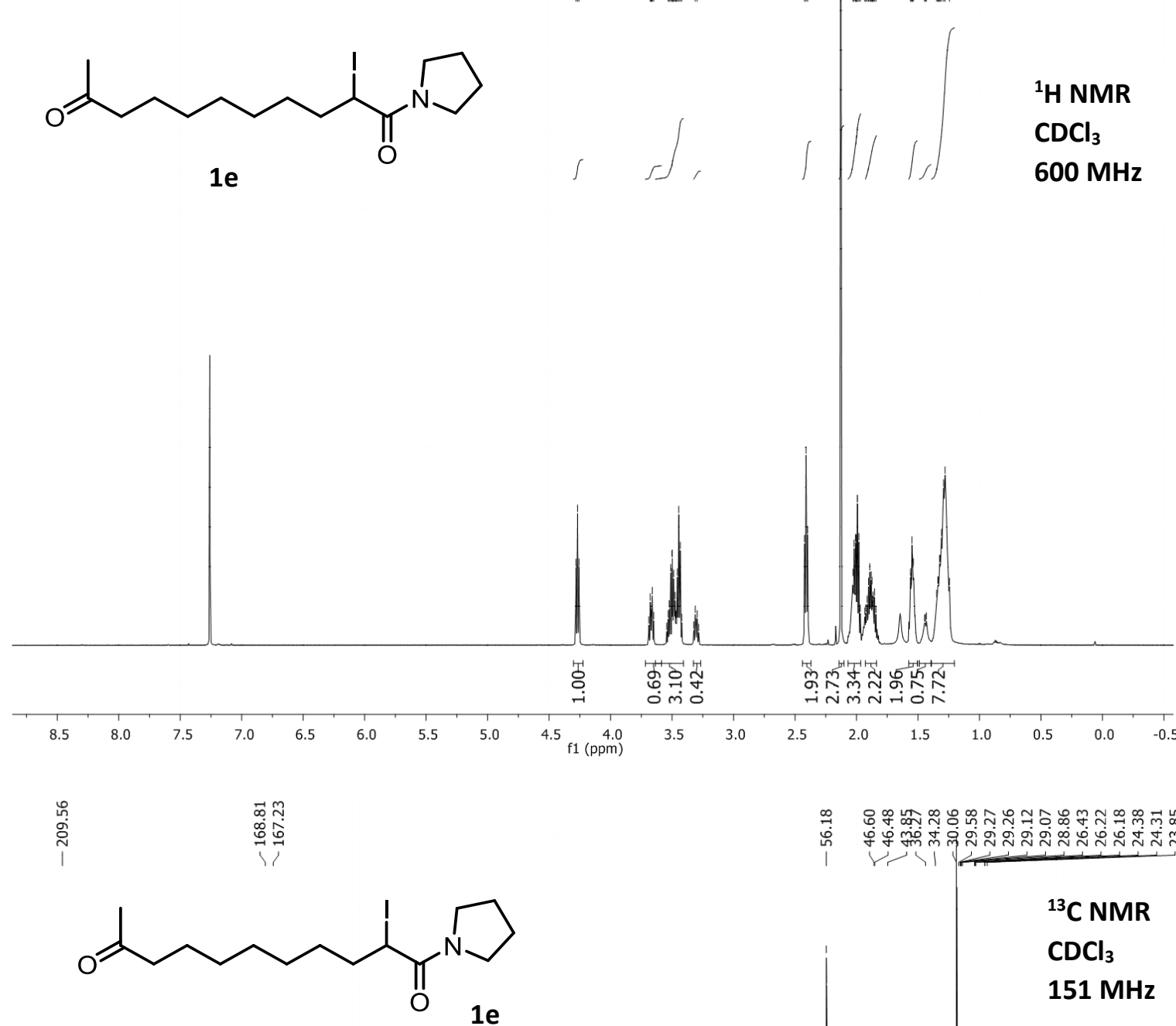

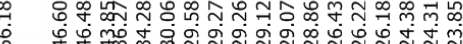

${ }^{13}$ C NMR

$\mathrm{CDCl}_{3}$

$151 \mathrm{MHz}$

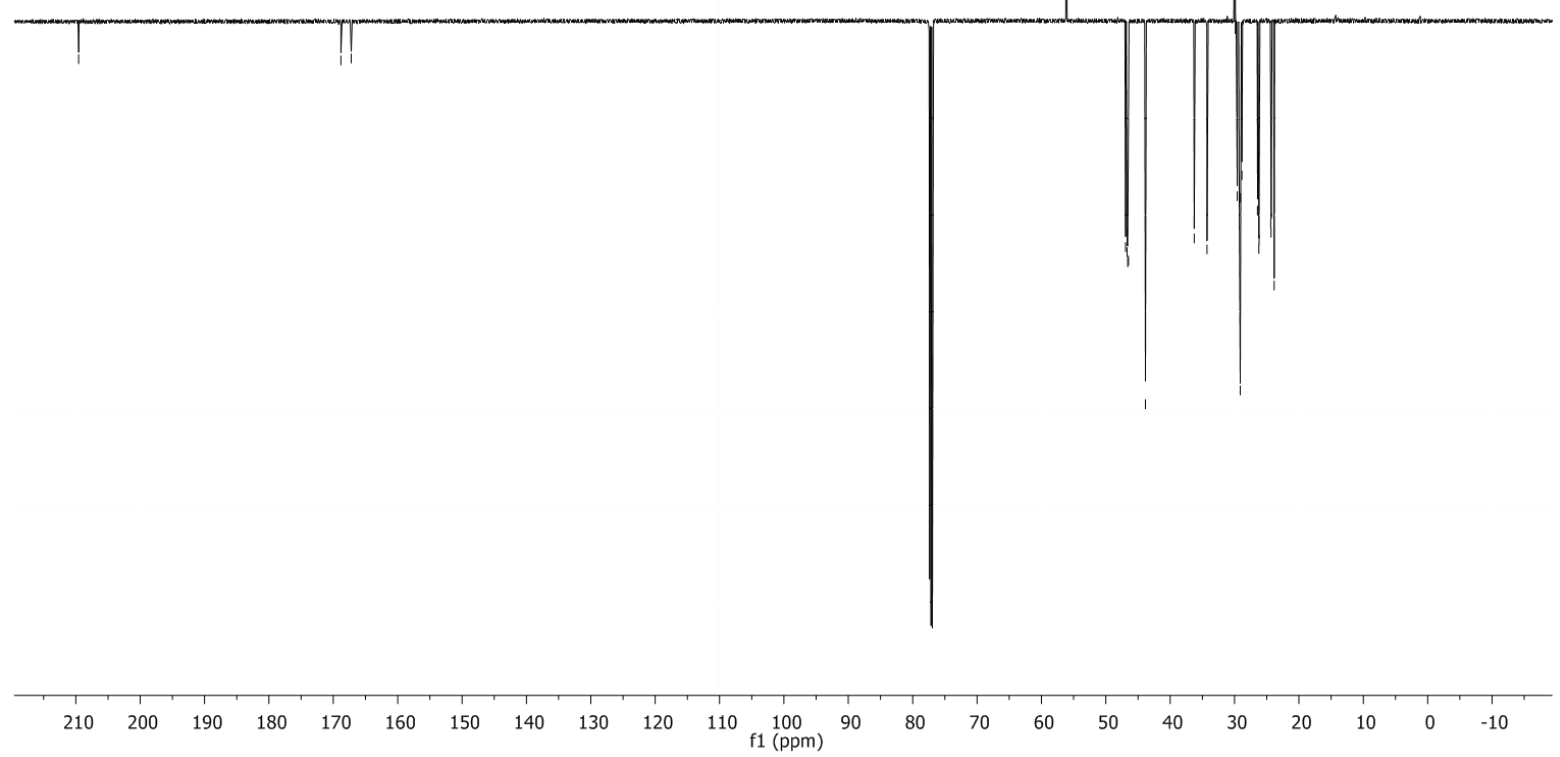




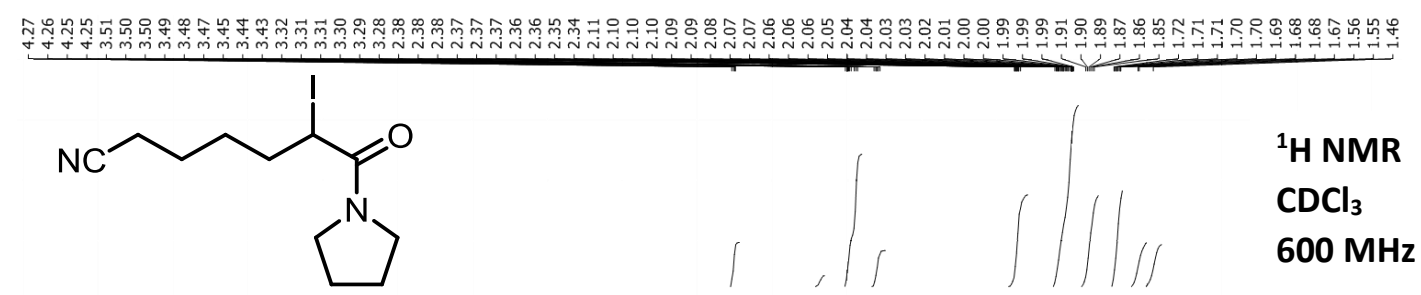

$1 f$
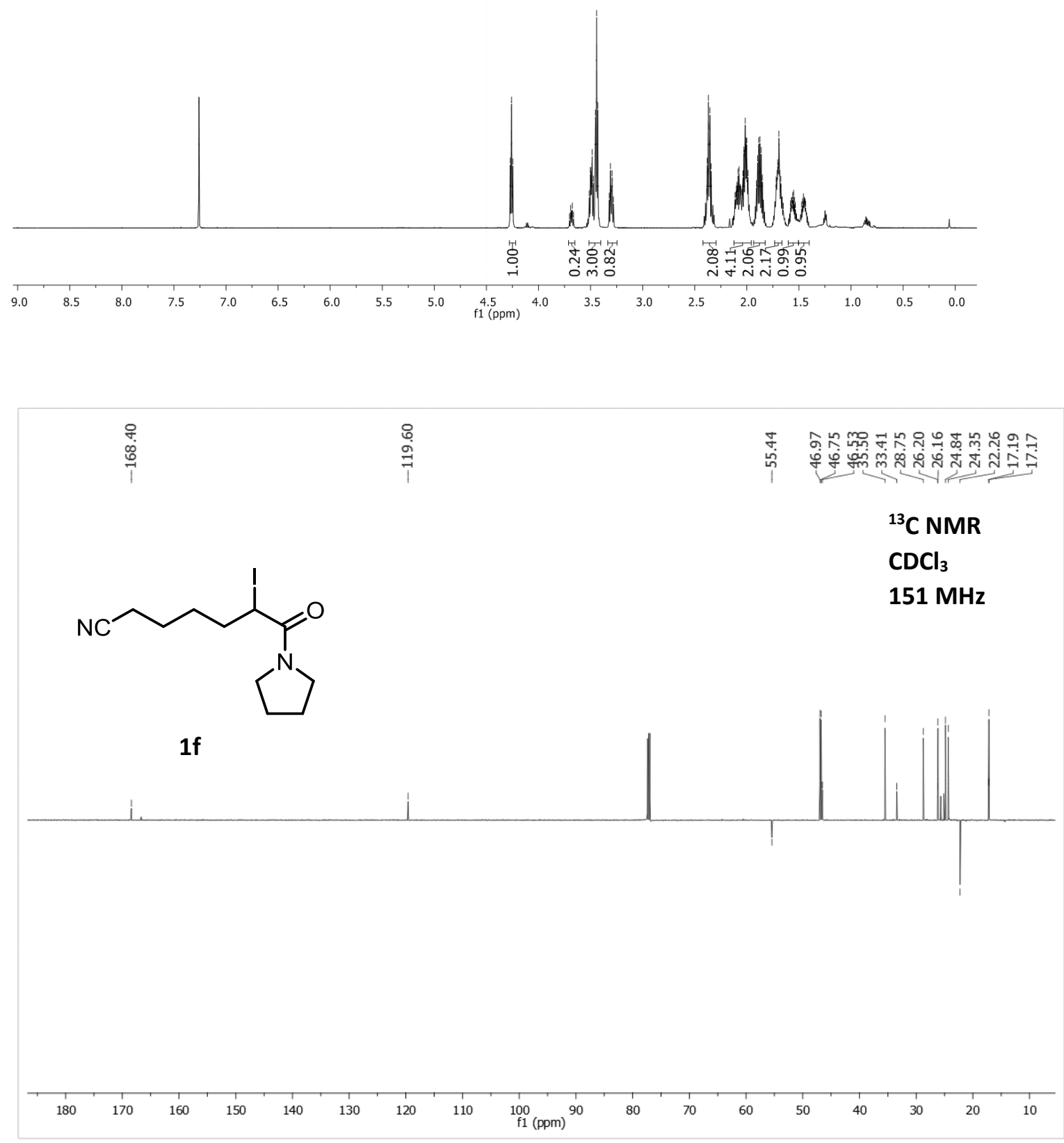


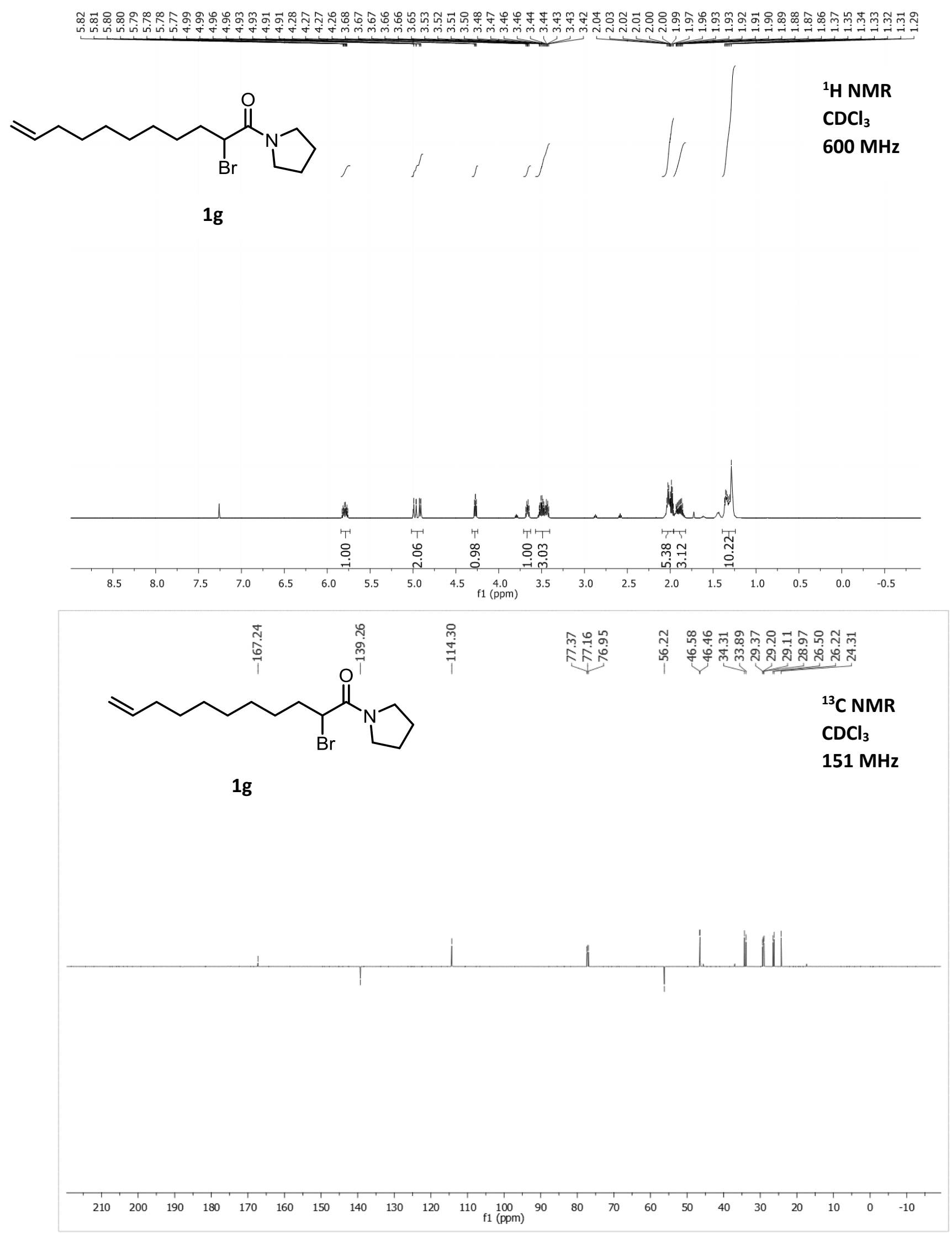


<smiles>C#CCCC(Cl)C(=O)N1CCCC1</smiles>

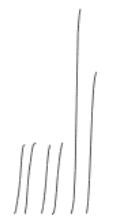

${ }^{1} \mathrm{H}$ NMR

$\mathrm{CDCl}_{3}$ $600 \mathrm{MHz}$

$1 \mathrm{~h}$

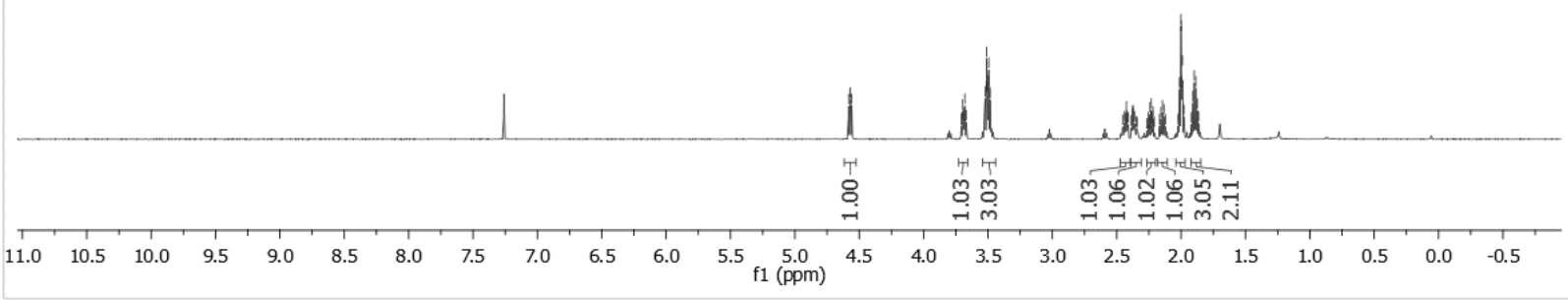<smiles>C#CCCC(Cl)C(=O)N1CCCC1</smiles>

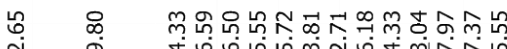

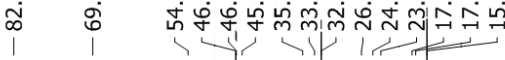

1h

${ }^{13}$ C NMR

$\mathrm{CDCl}_{3}$

$151 \mathrm{MHz}$

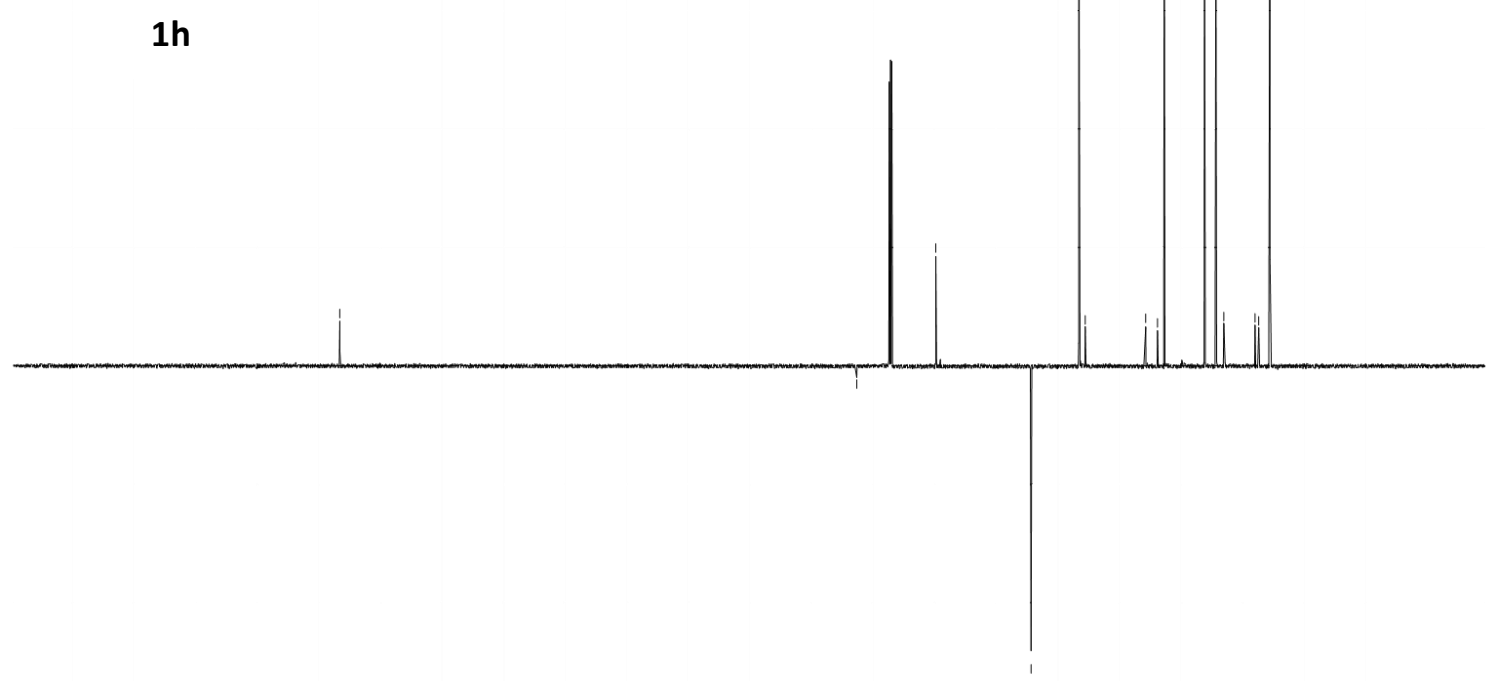

$\begin{array}{llllllllllllllllllllllll}210 & 200 & 190 & 180 & 170 & 160 & 150 & 140 & 130 & 120 & 110 & \underset{\mathrm{f} 1}{(\mathrm{ppm})} & 90 & 80 & 70 & 60 & 50 & 40 & 30 & 20 & 10 & 0 & -10\end{array}$ 


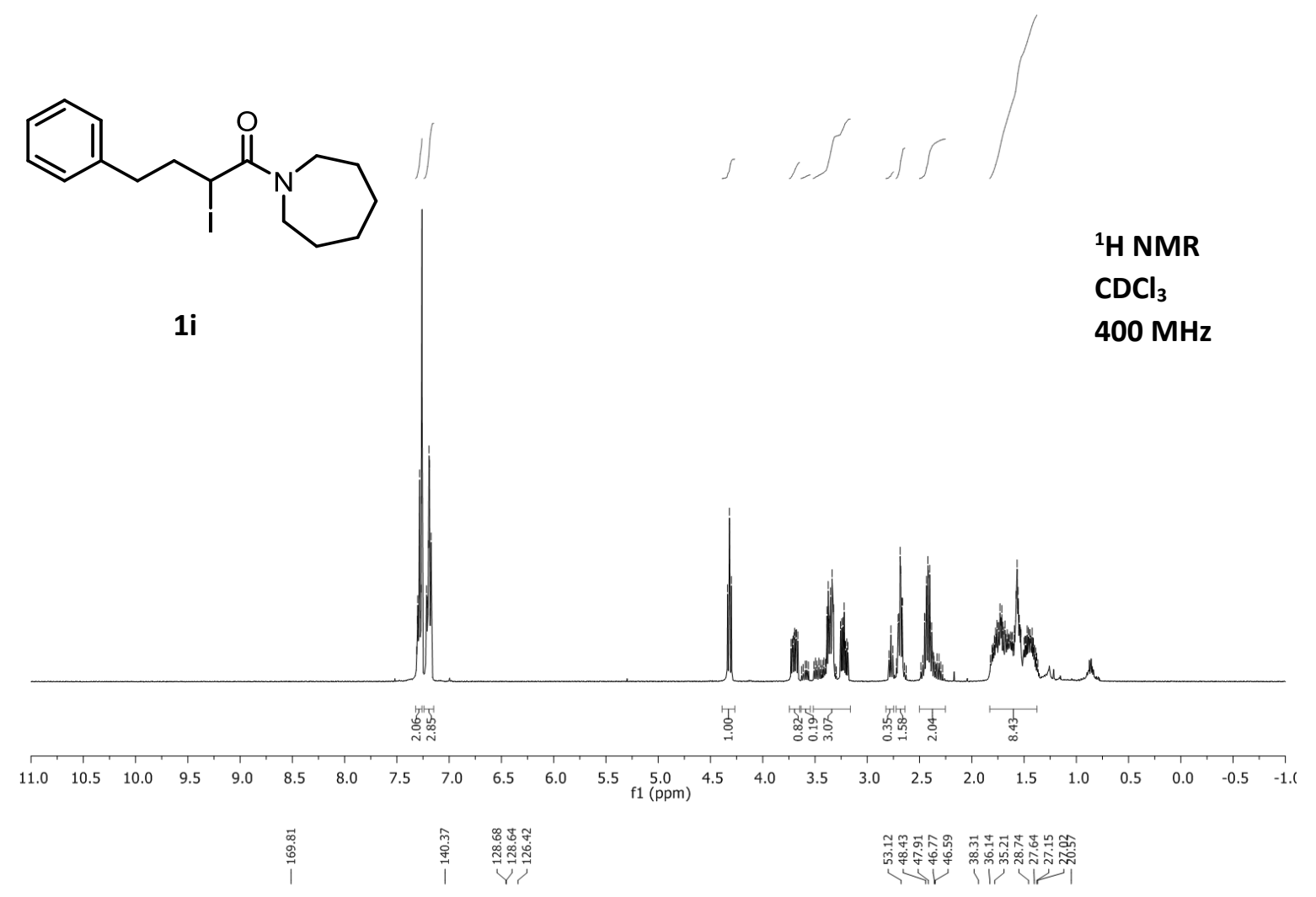<smiles>O=C(C(I)CCc1ccccc1)N1CCCCCC1</smiles>

${ }^{13} \mathrm{CNMR}$

$\mathrm{CDCl}_{3}$ $100 \mathrm{MHz}$

$1 i$

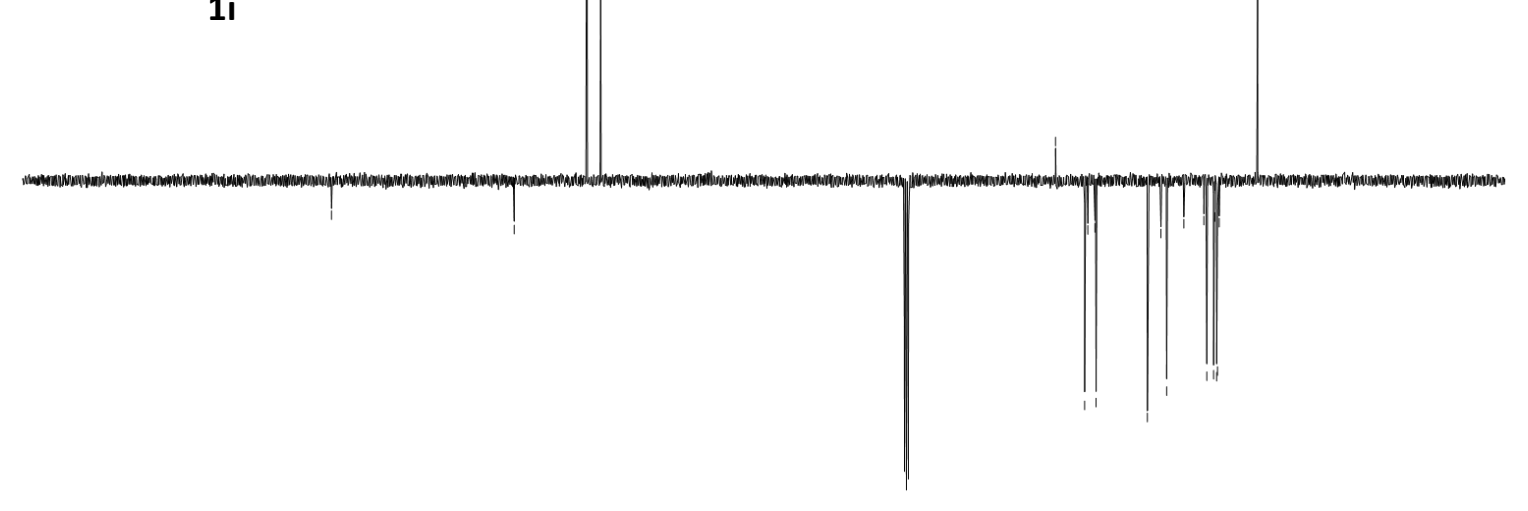

$\begin{array}{llllllllllllllllllllllll}210 & 200 & 190 & 180 & 170 & 160 & 150 & 140 & 130 & 120 & 110 & \begin{array}{c}100 \\ \mathrm{f} 1\end{array}(\mathrm{ppm}) & 90 & 80 & 70 & 60 & 50 & 40 & 30 & 20 & 10 & 0 & -10\end{array}$ 


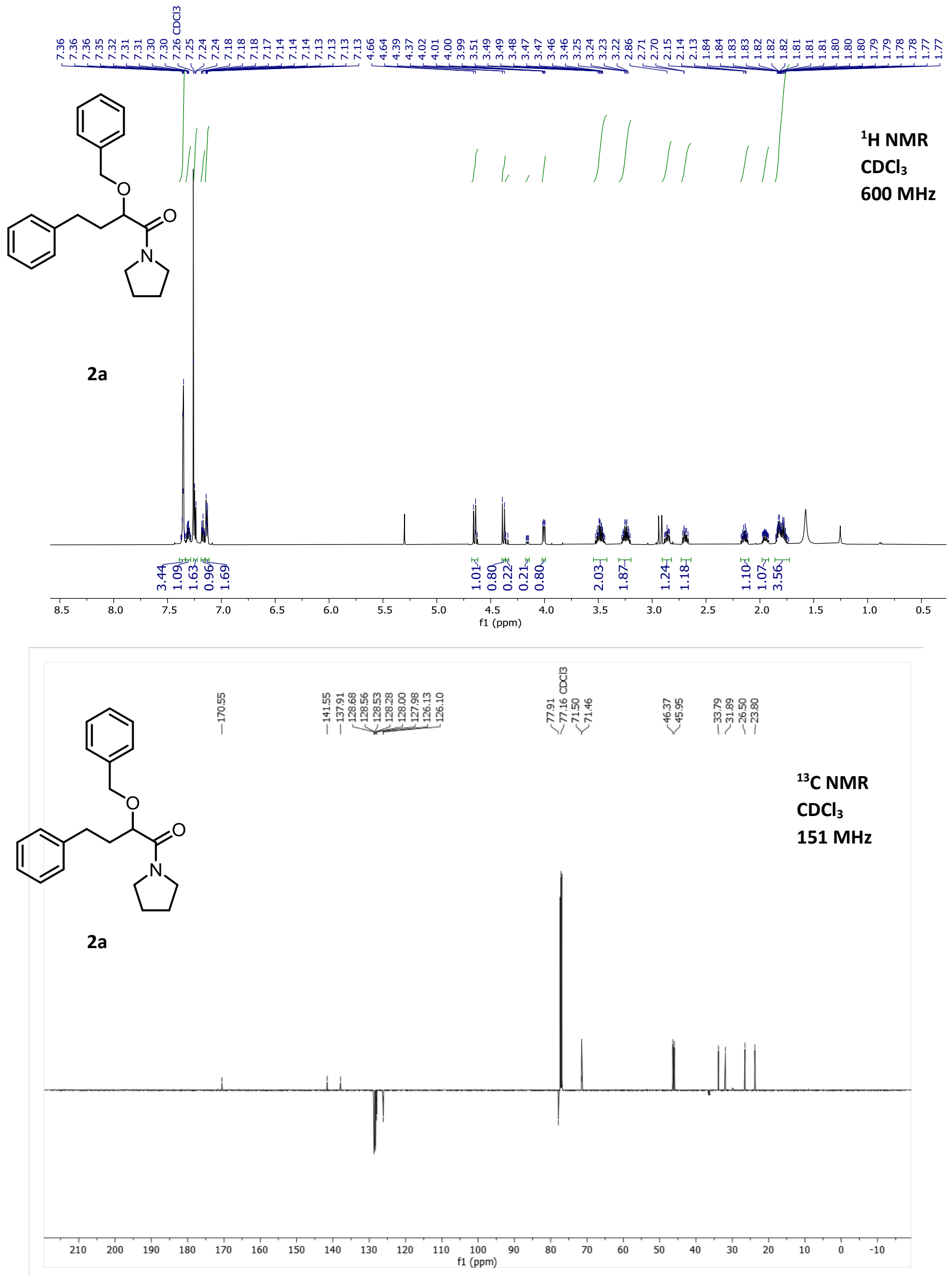




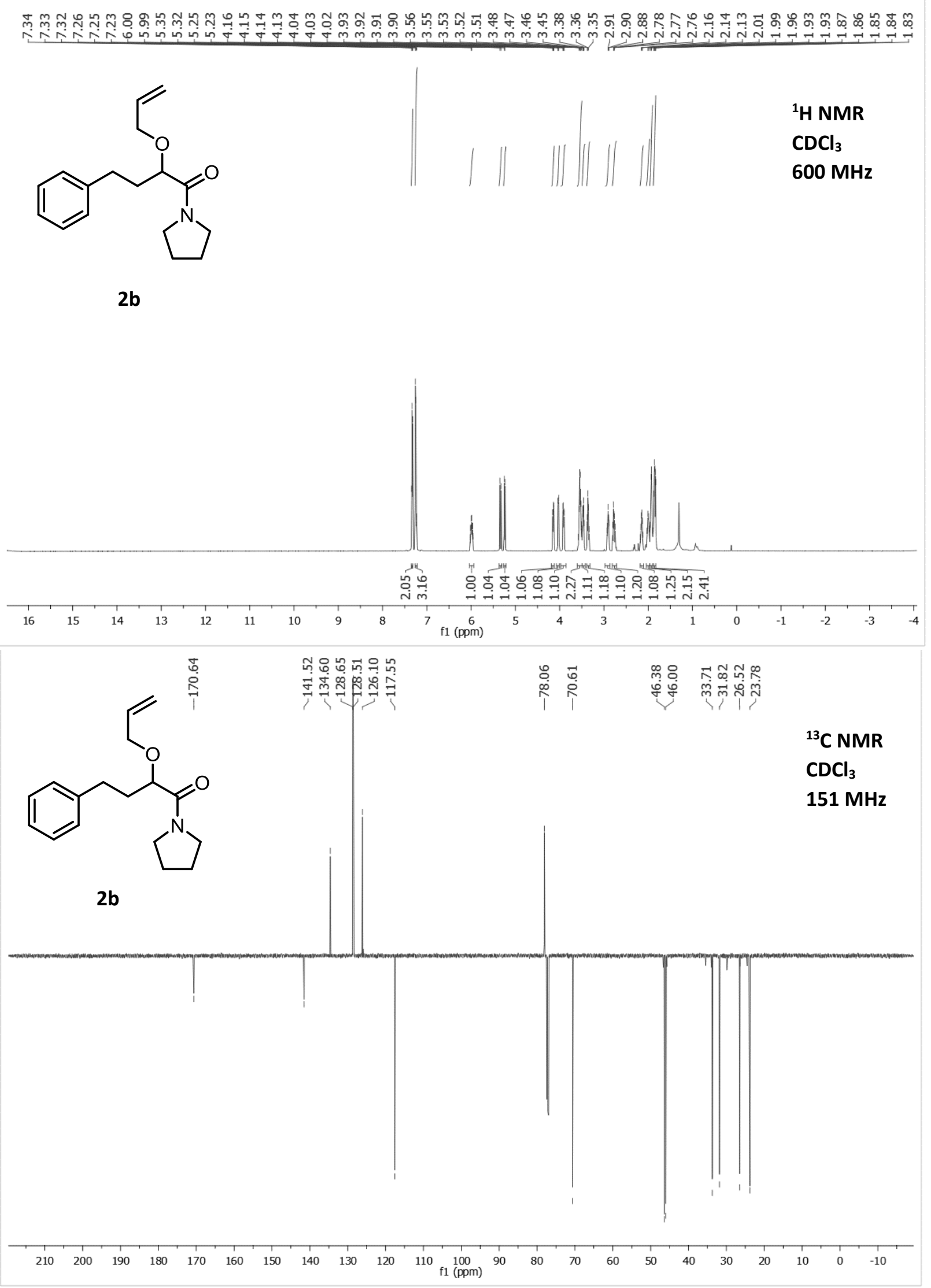




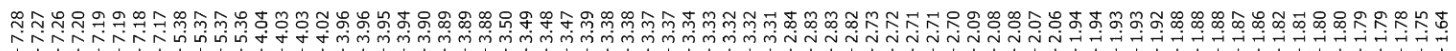
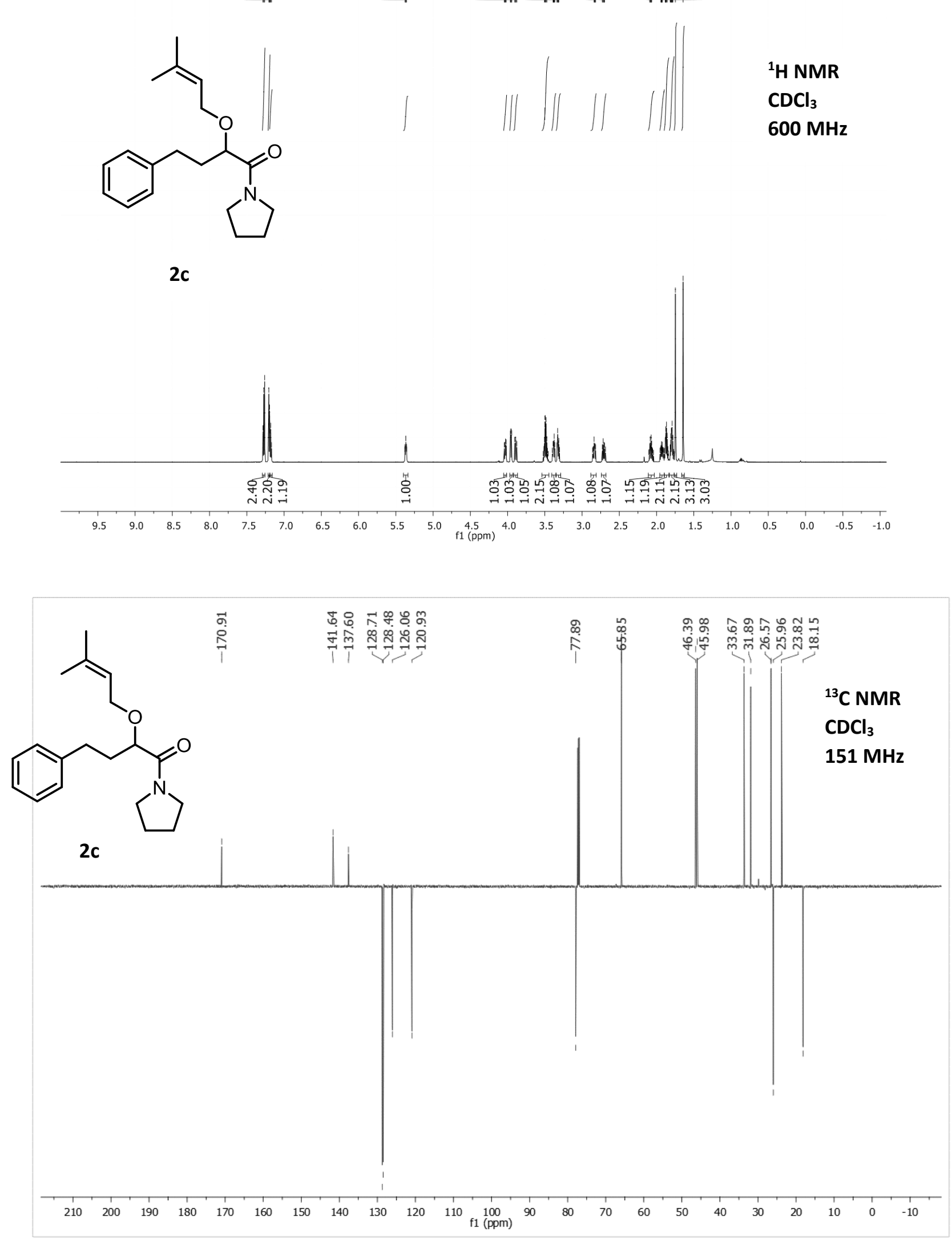


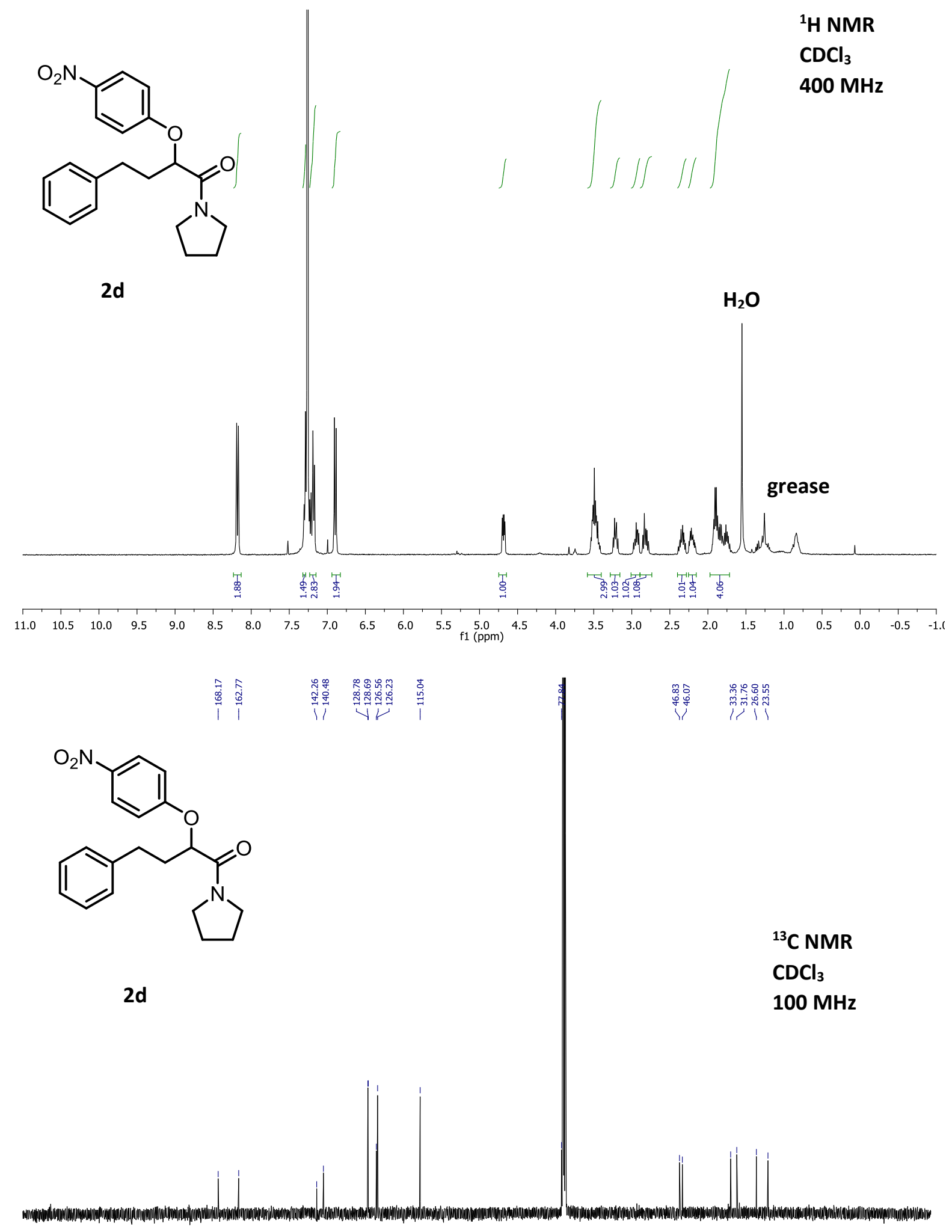

$\begin{array}{lllllllllllllllllllllllll}210 & 200 & 190 & 180 & 170 & 160 & 150 & 140 & 130 & 120 & 110 & \underset{\mathrm{f} 1}{100}(\mathrm{ppm}) & 90 & 80 & 70 & 60 & 50 & 40 & 30 & 20 & 10 & 0 & -10\end{array}$ 


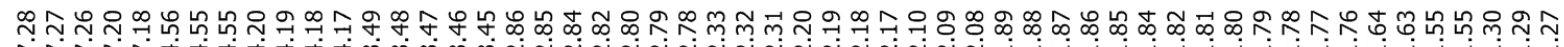

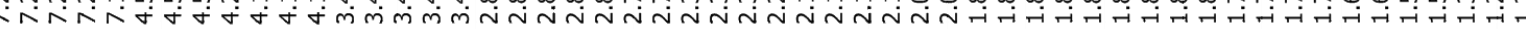
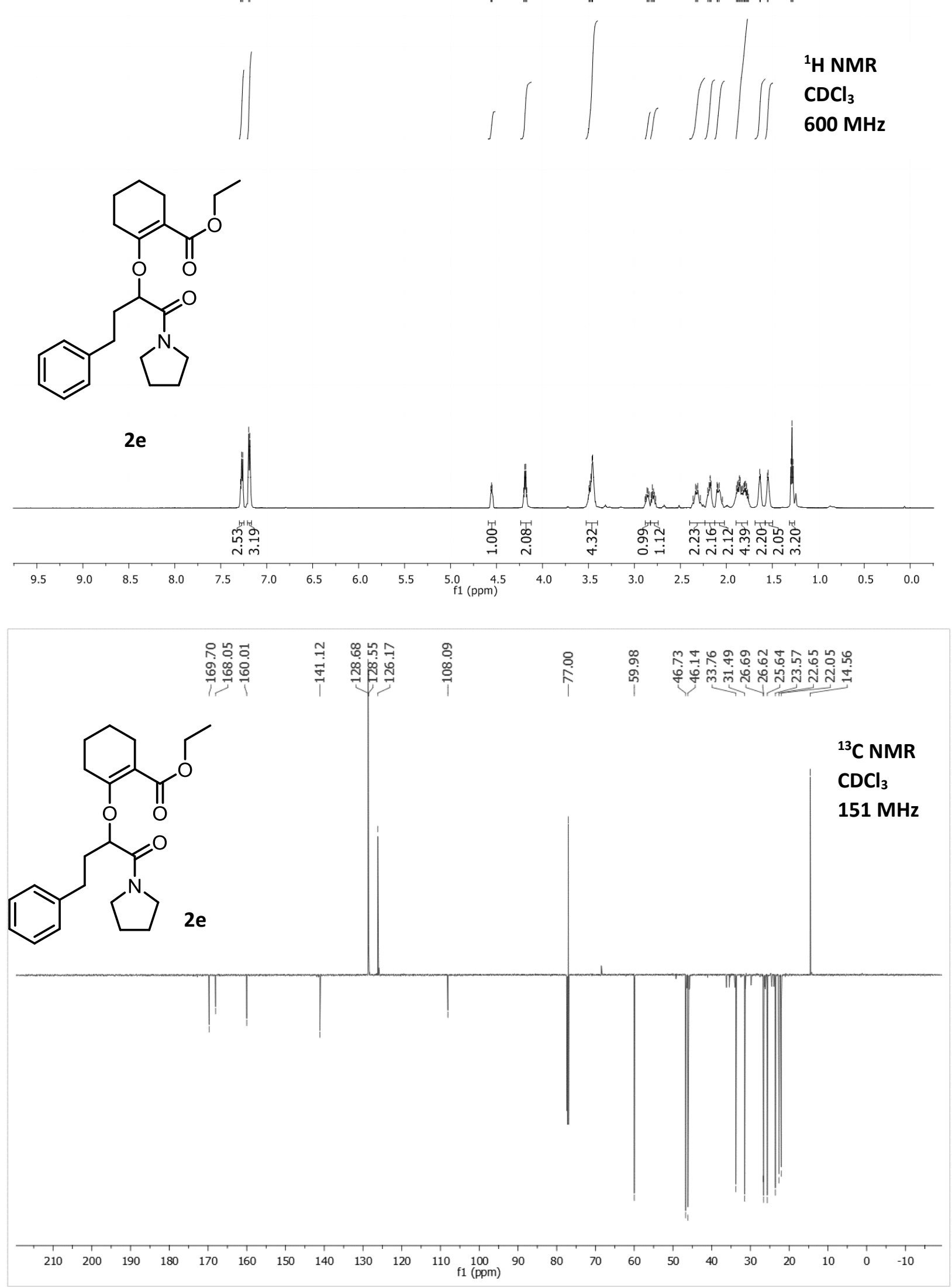


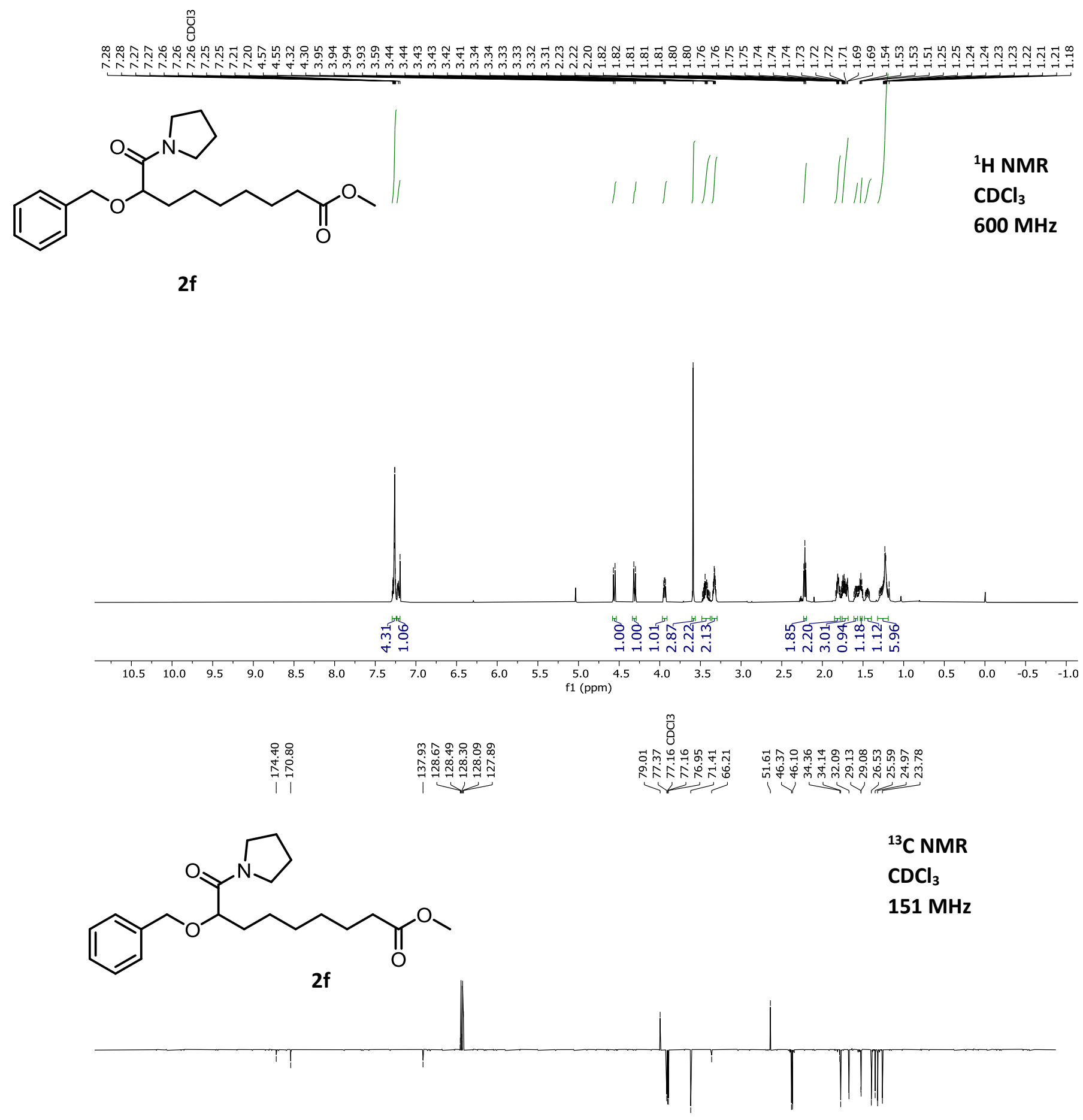

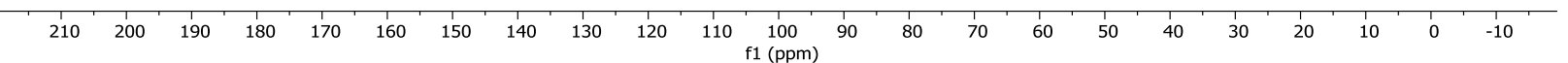




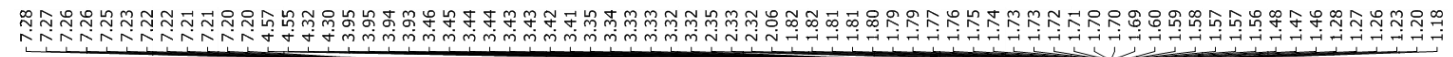
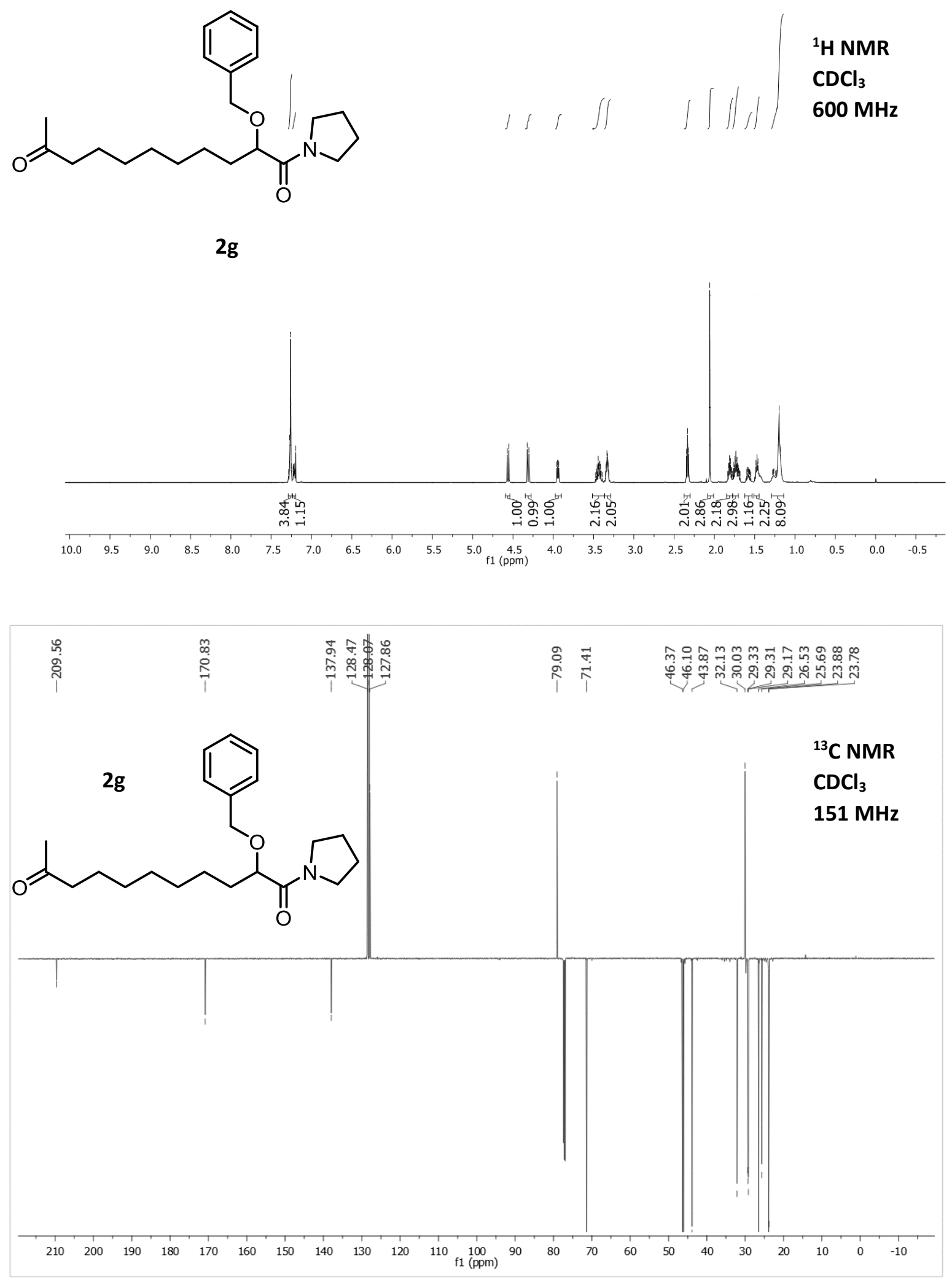


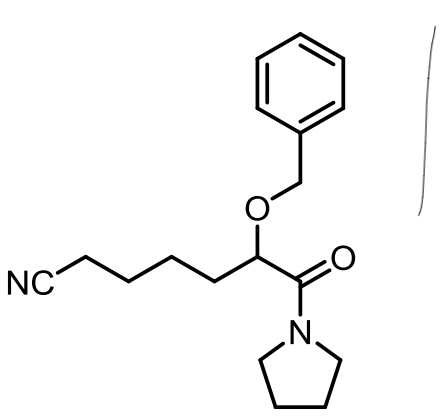

${ }^{1} \mathrm{H}$ NMR

$\mathrm{CDCl}_{3}$

$600 \mathrm{MHz}$

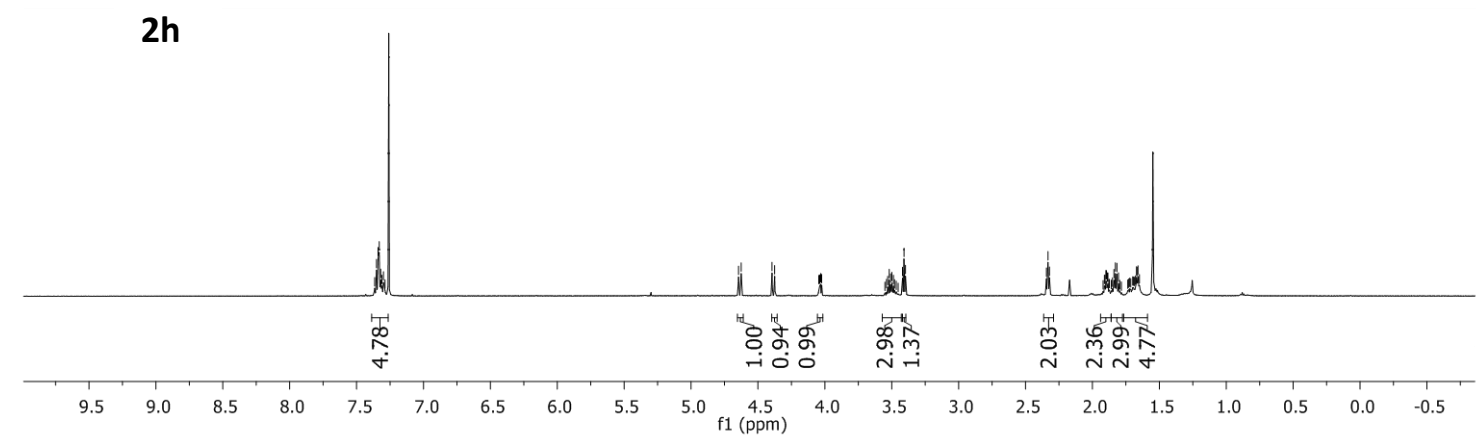

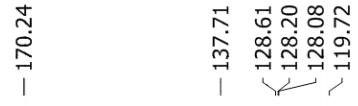

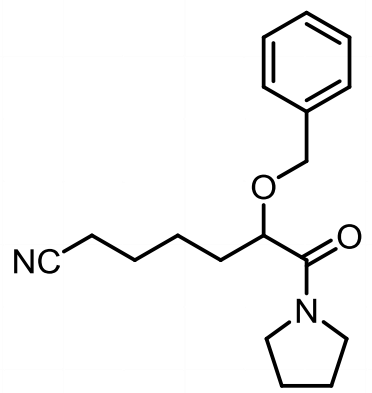

$2 \mathrm{~h}$

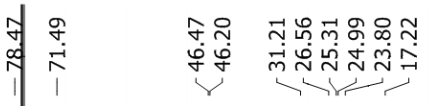

${ }^{13}$ C NMR

$\mathrm{CDCl}_{3}$

$151 \mathrm{MHz}$

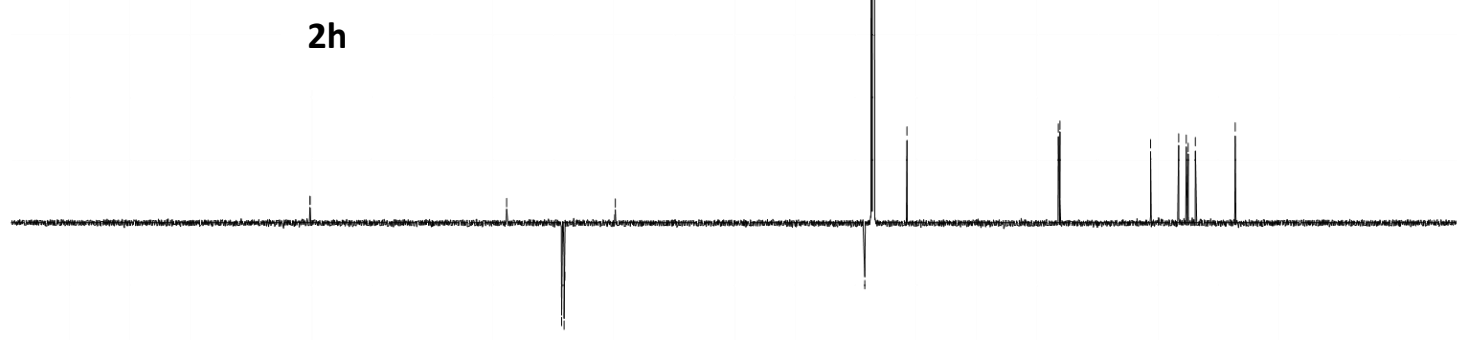

$\begin{array}{llllllllllllllllllllllllllllll}210 & 200 & 190 & 180 & 170 & 160 & 150 & 140 & 130 & 120 & 110 & 100 & 90 & 80 & 70 & 60 & 50 & 40 & 30 & 20 & 10 & 0 & -10\end{array}$ 

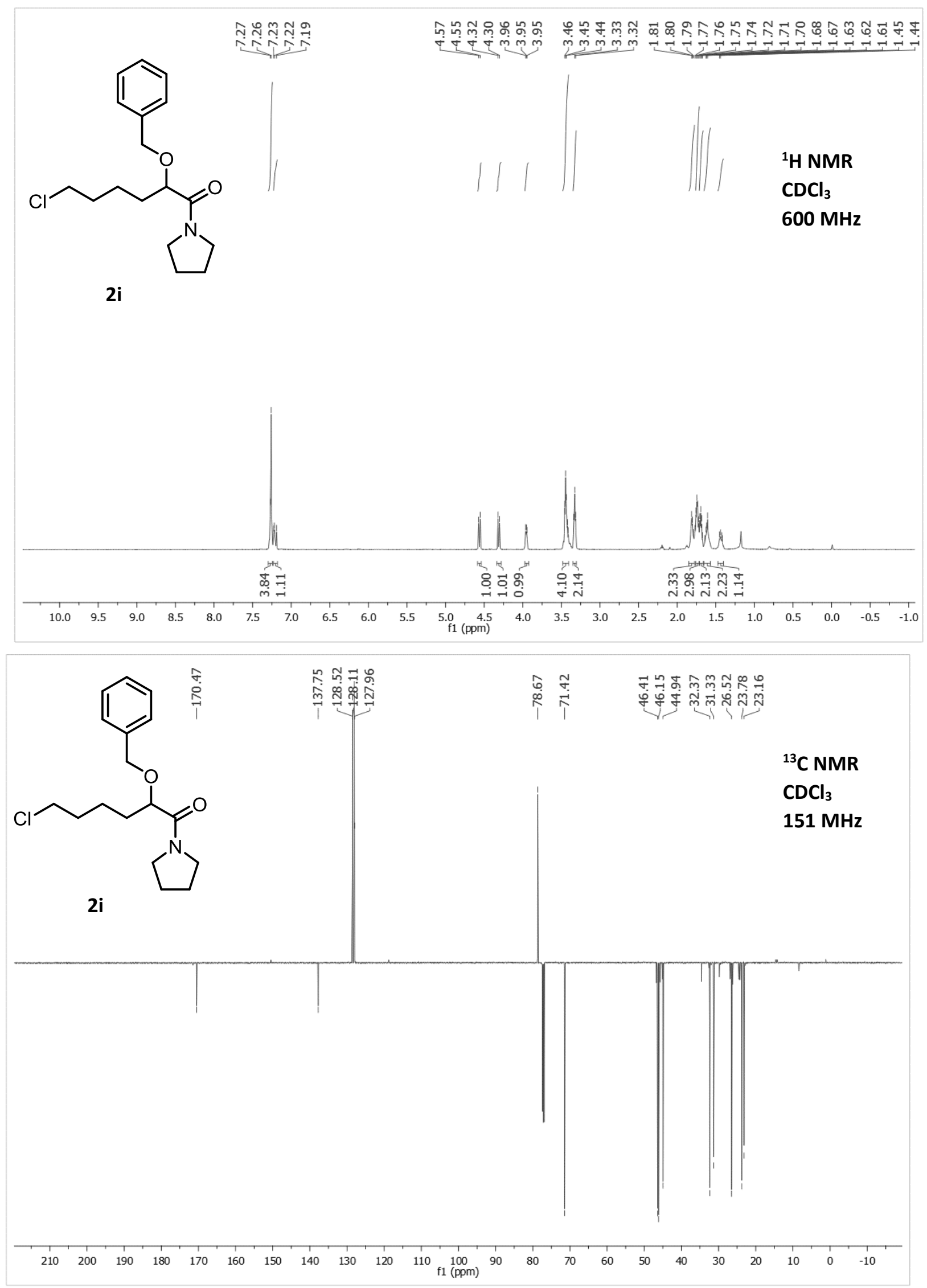

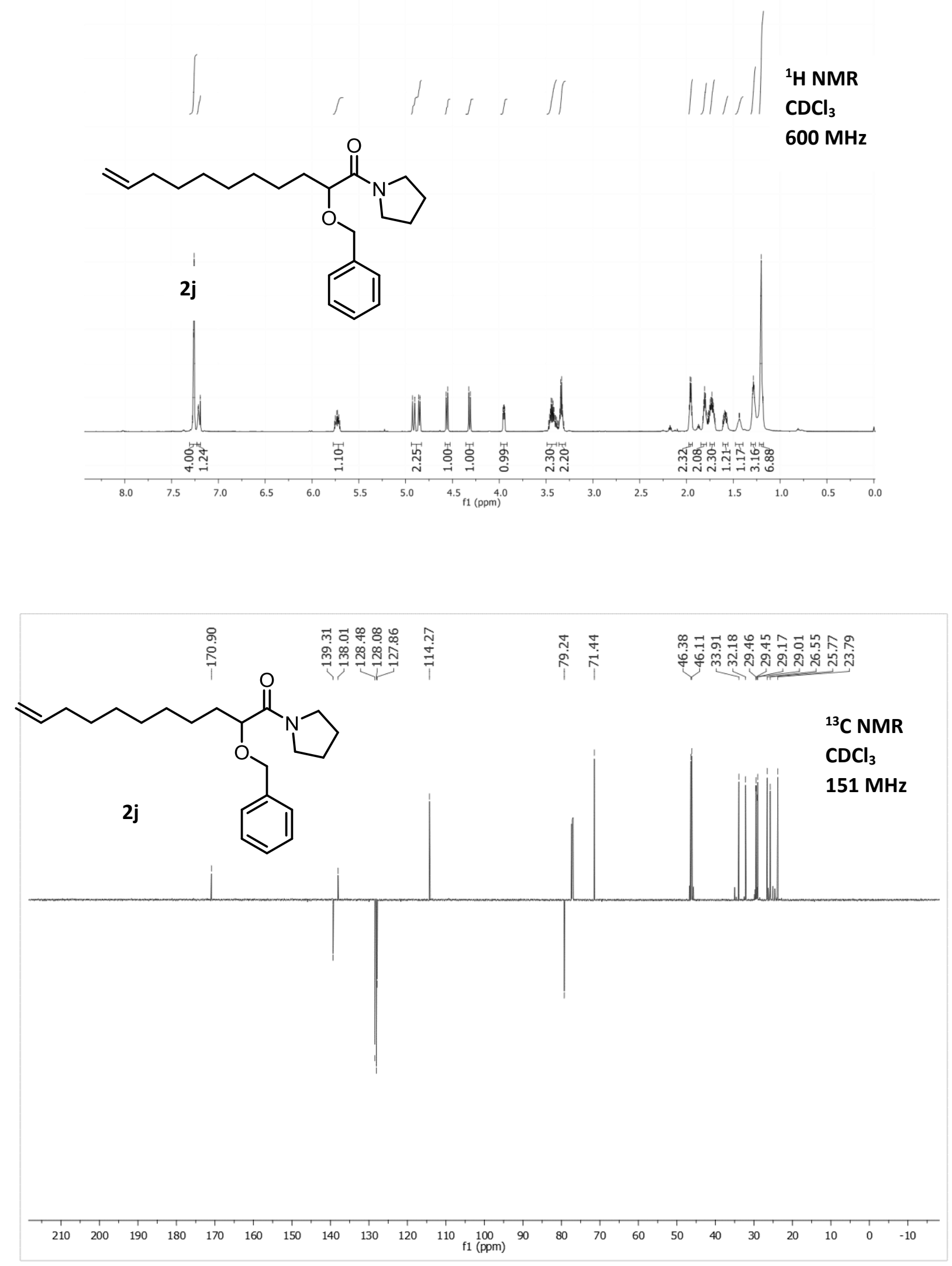


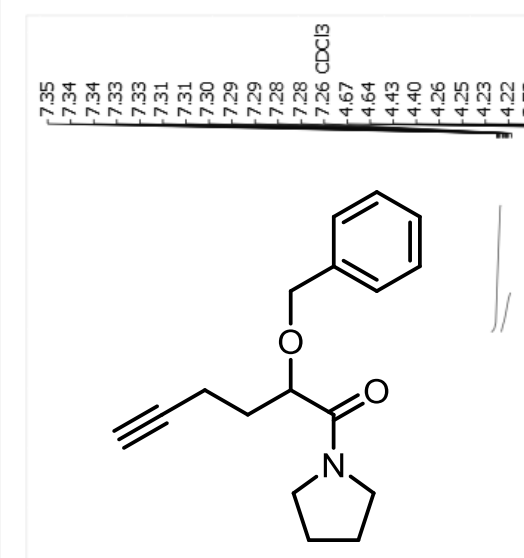

2k

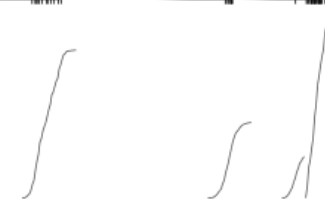

${ }^{1} \mathrm{H}$ NMR

$\mathrm{CDCl}_{3}$

$400 \mathrm{MHz}$

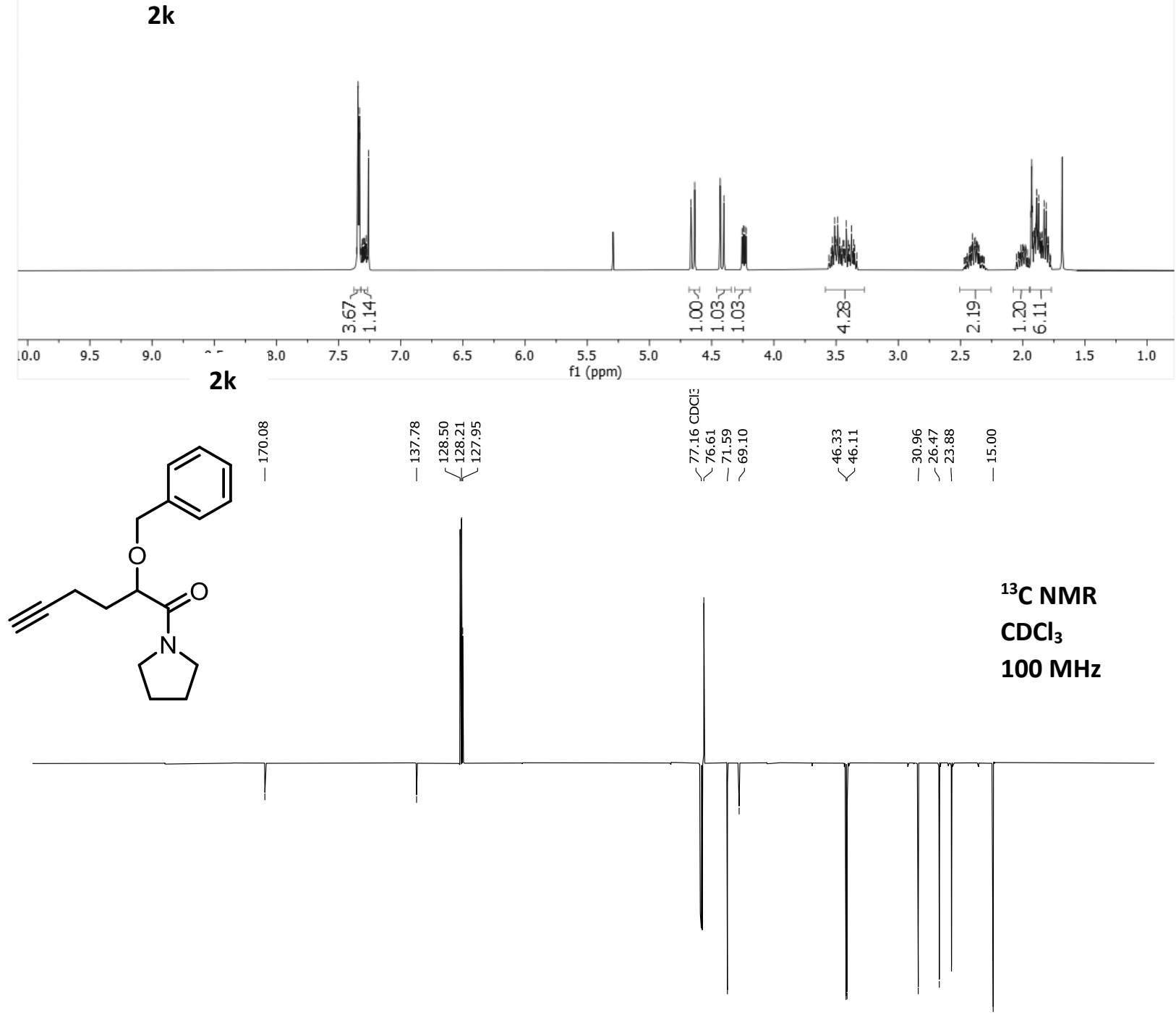

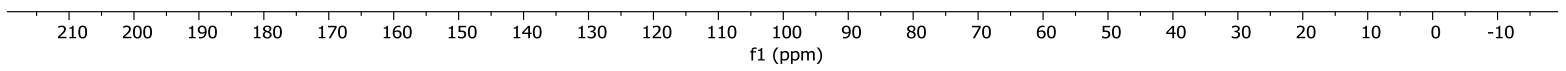




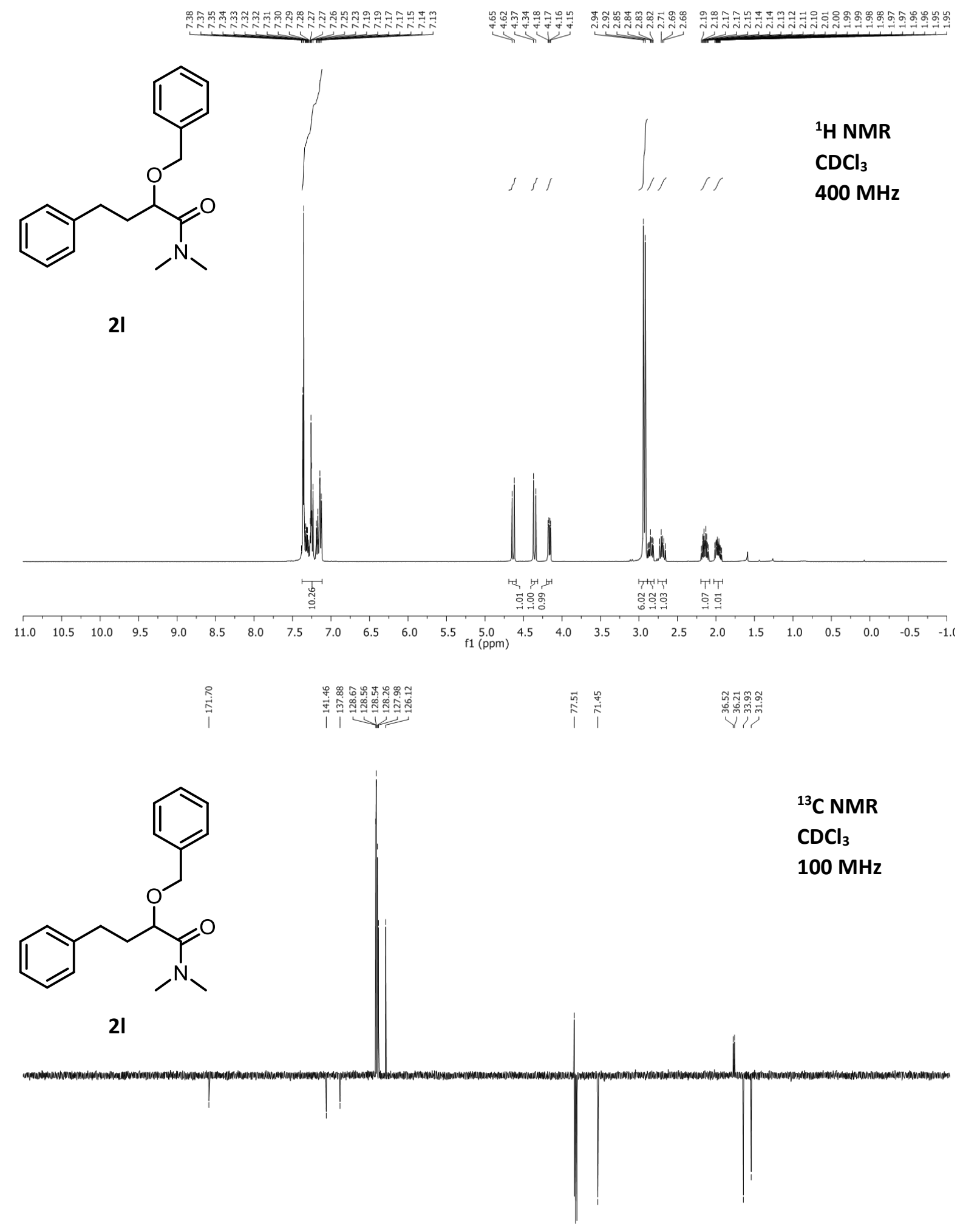

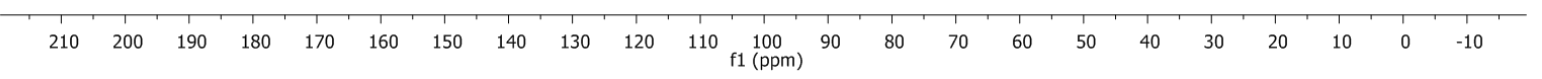



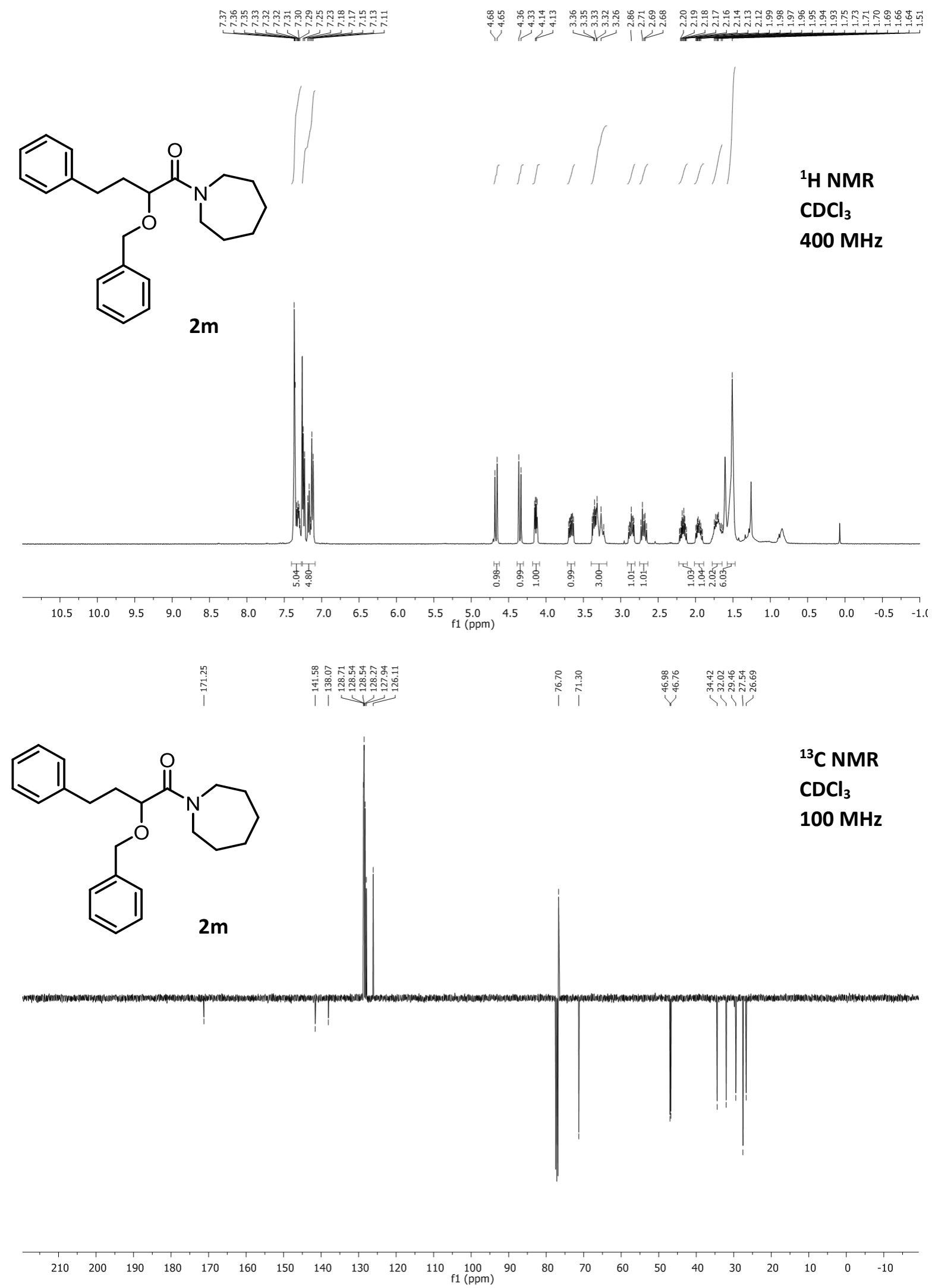


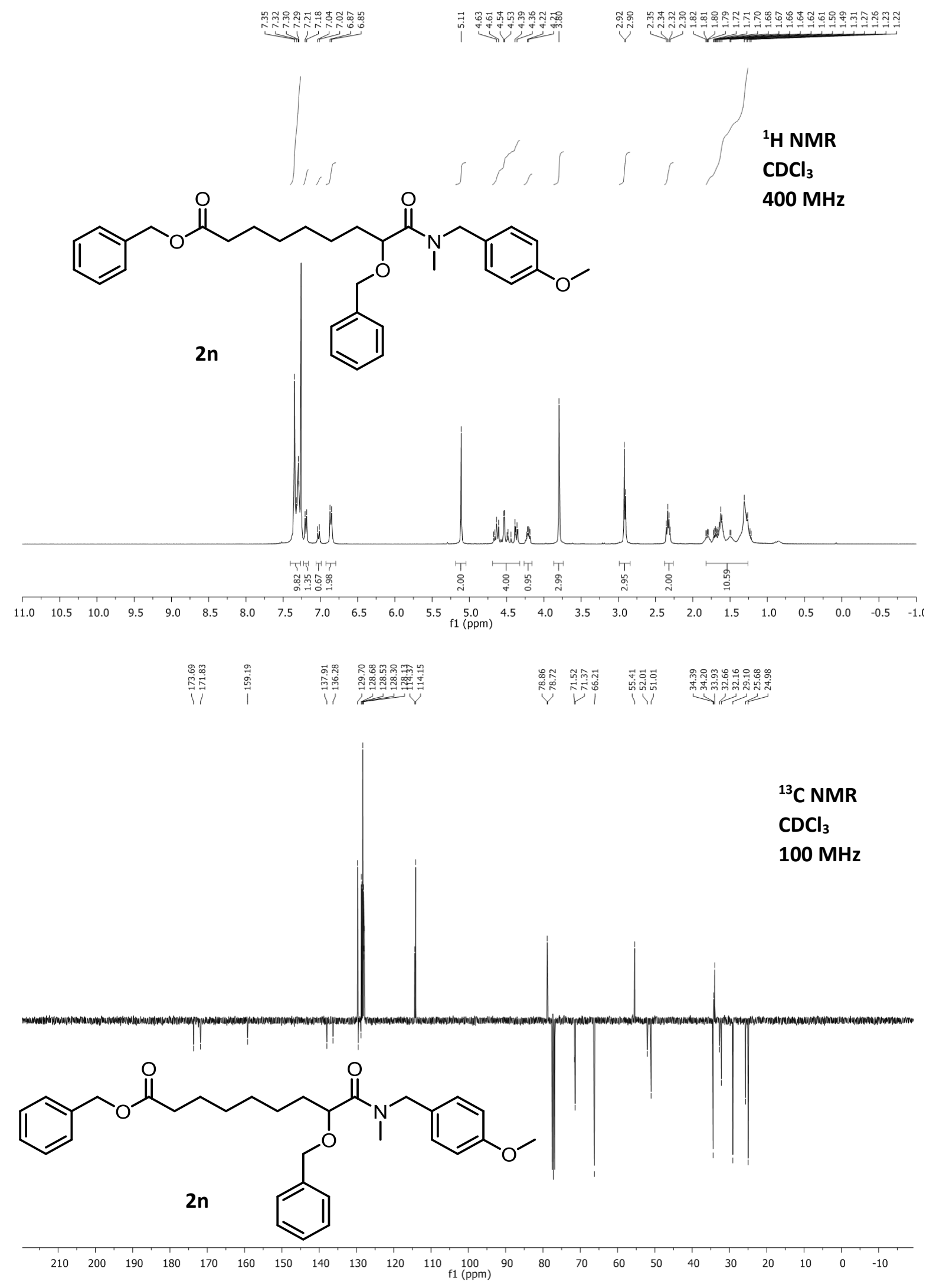




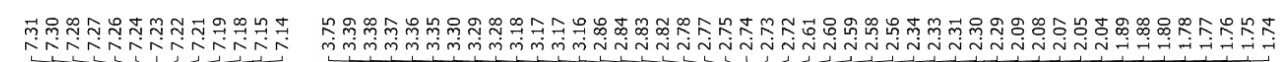
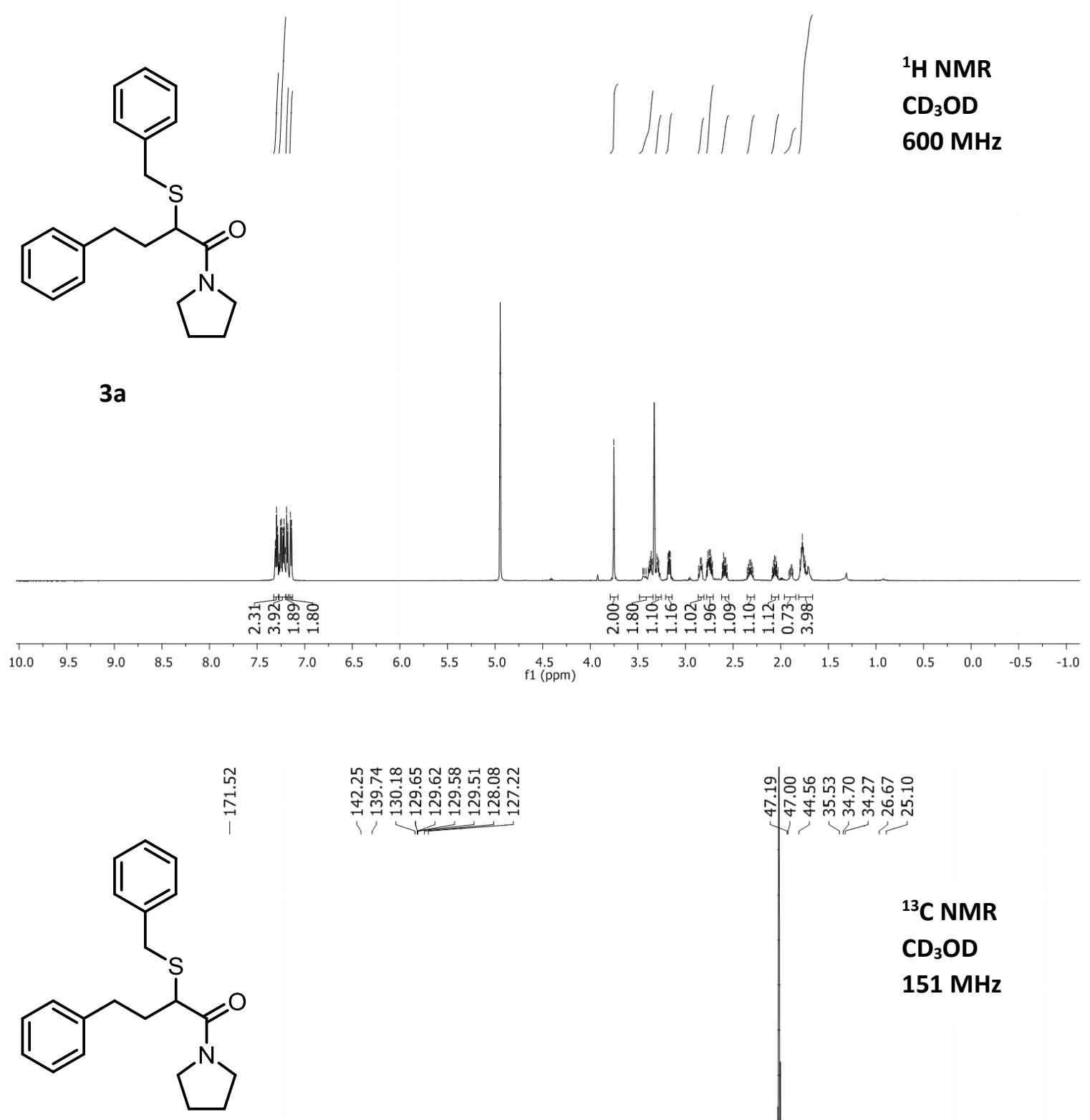

$3 a$

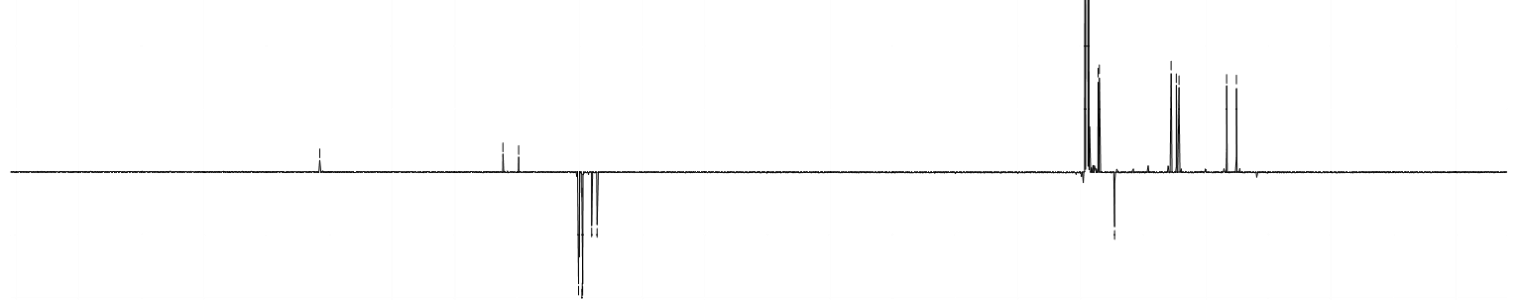

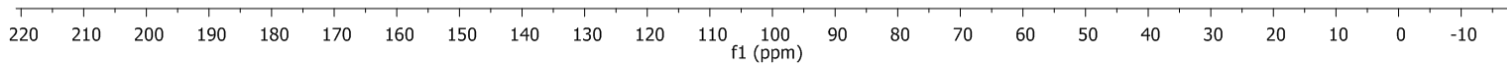



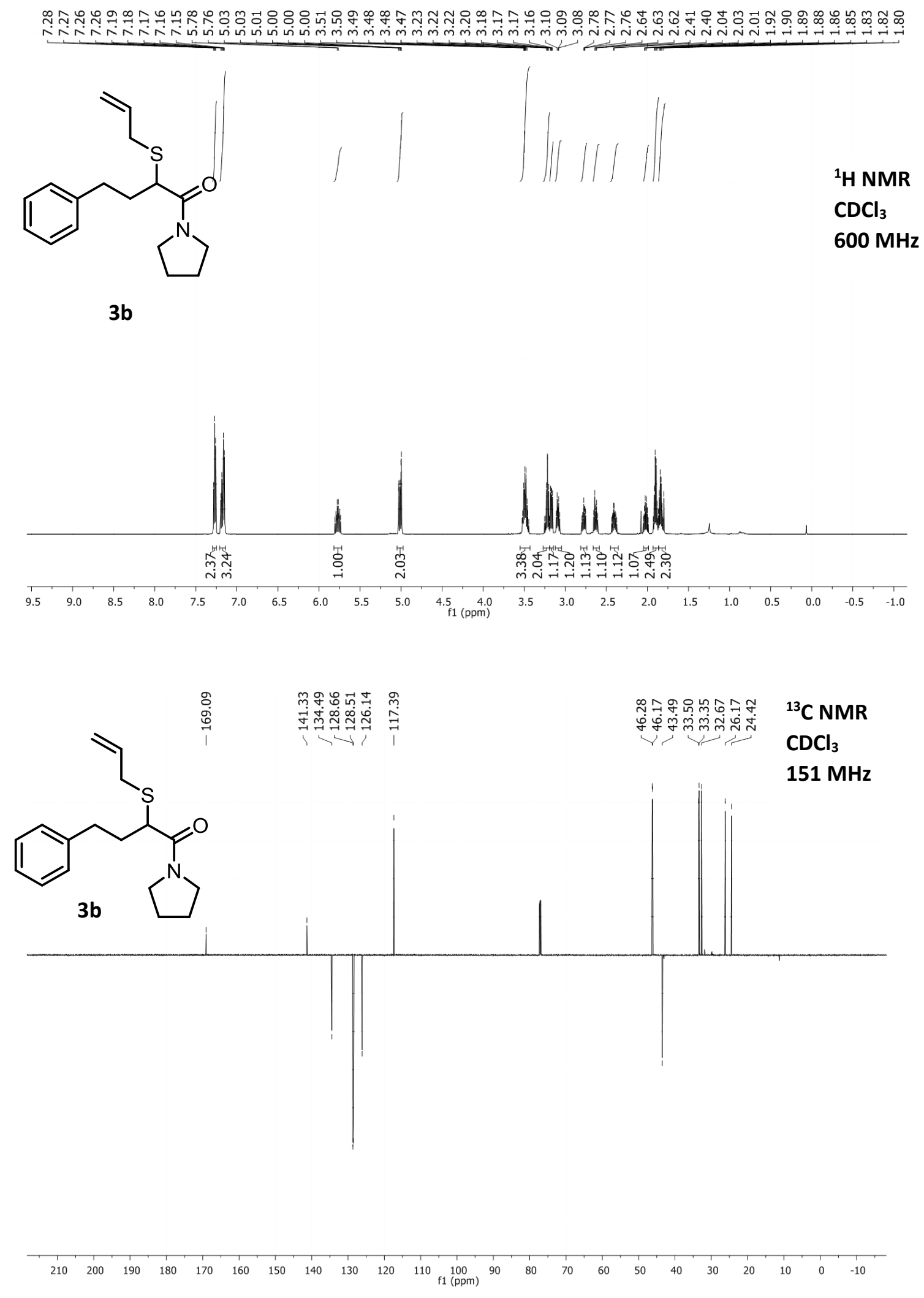


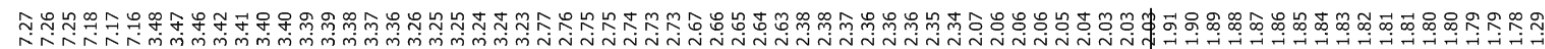
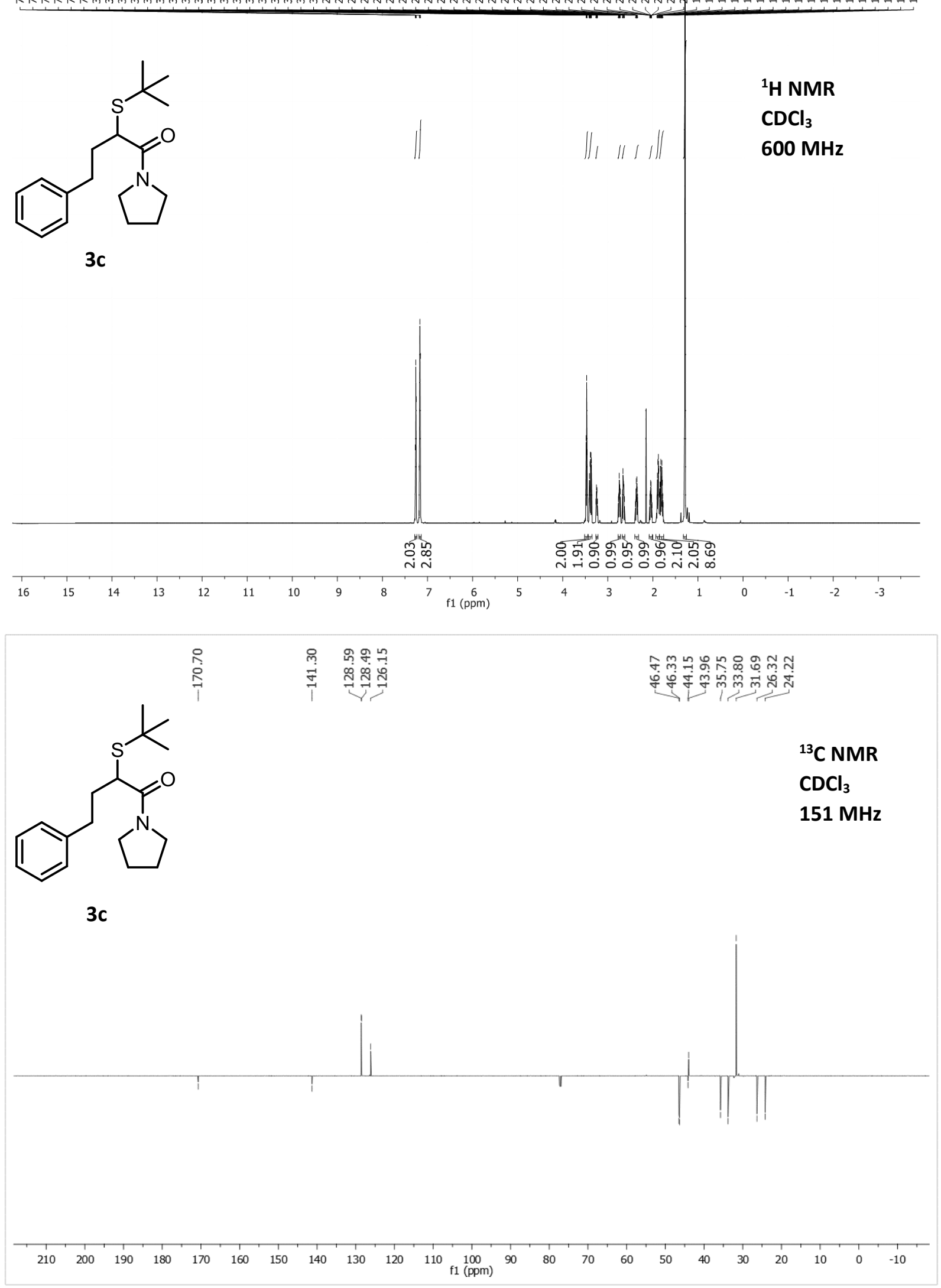

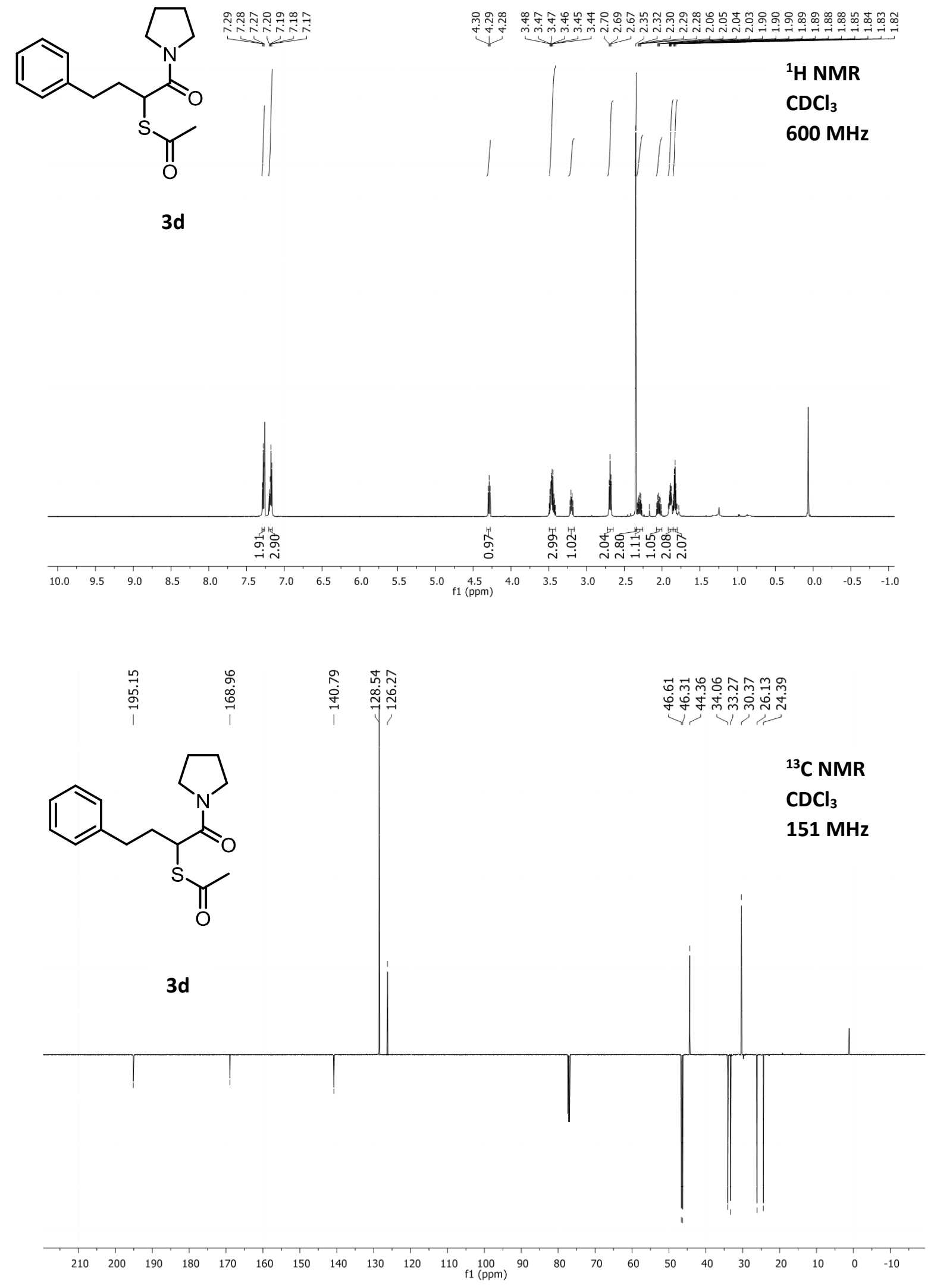

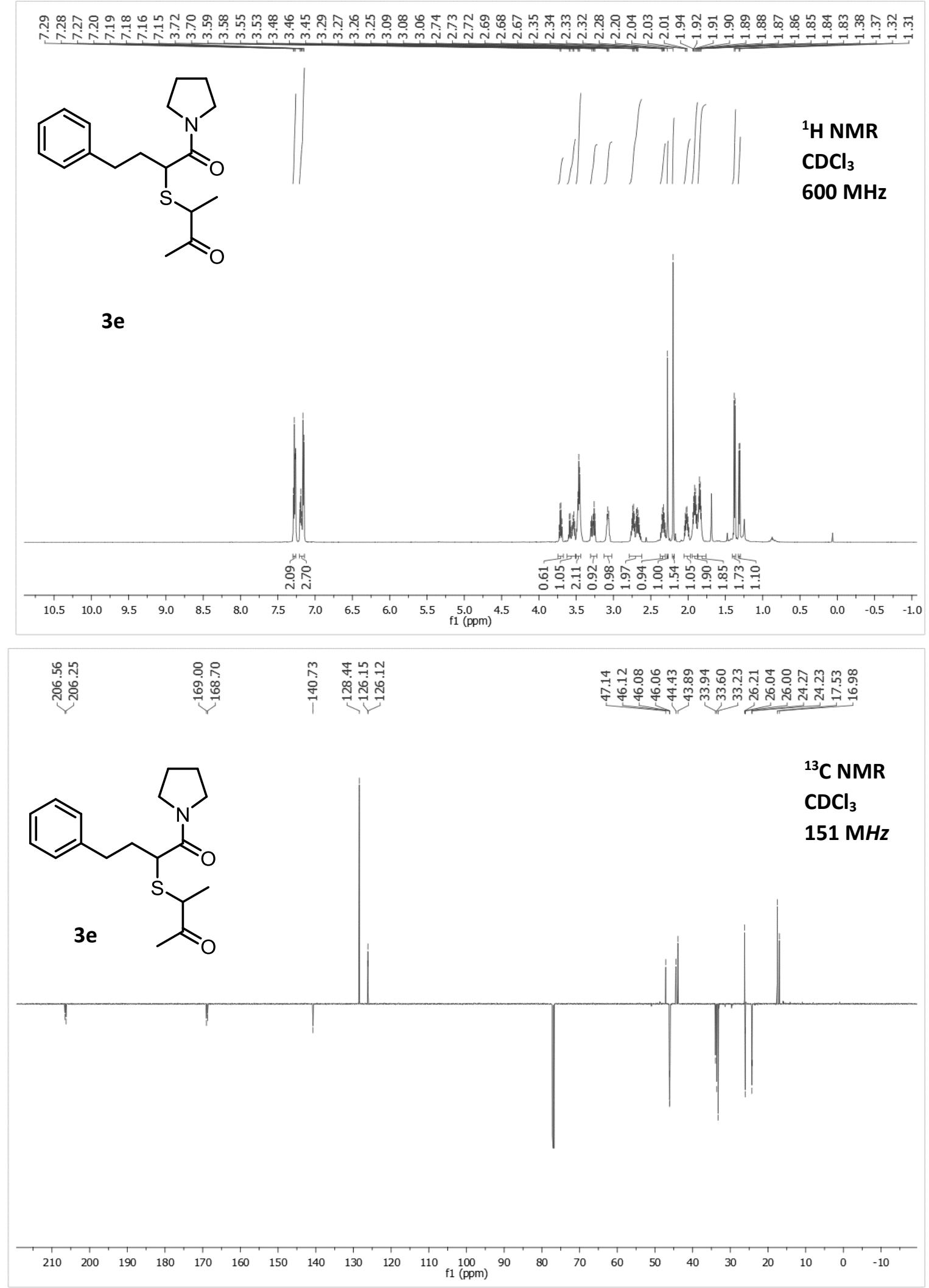


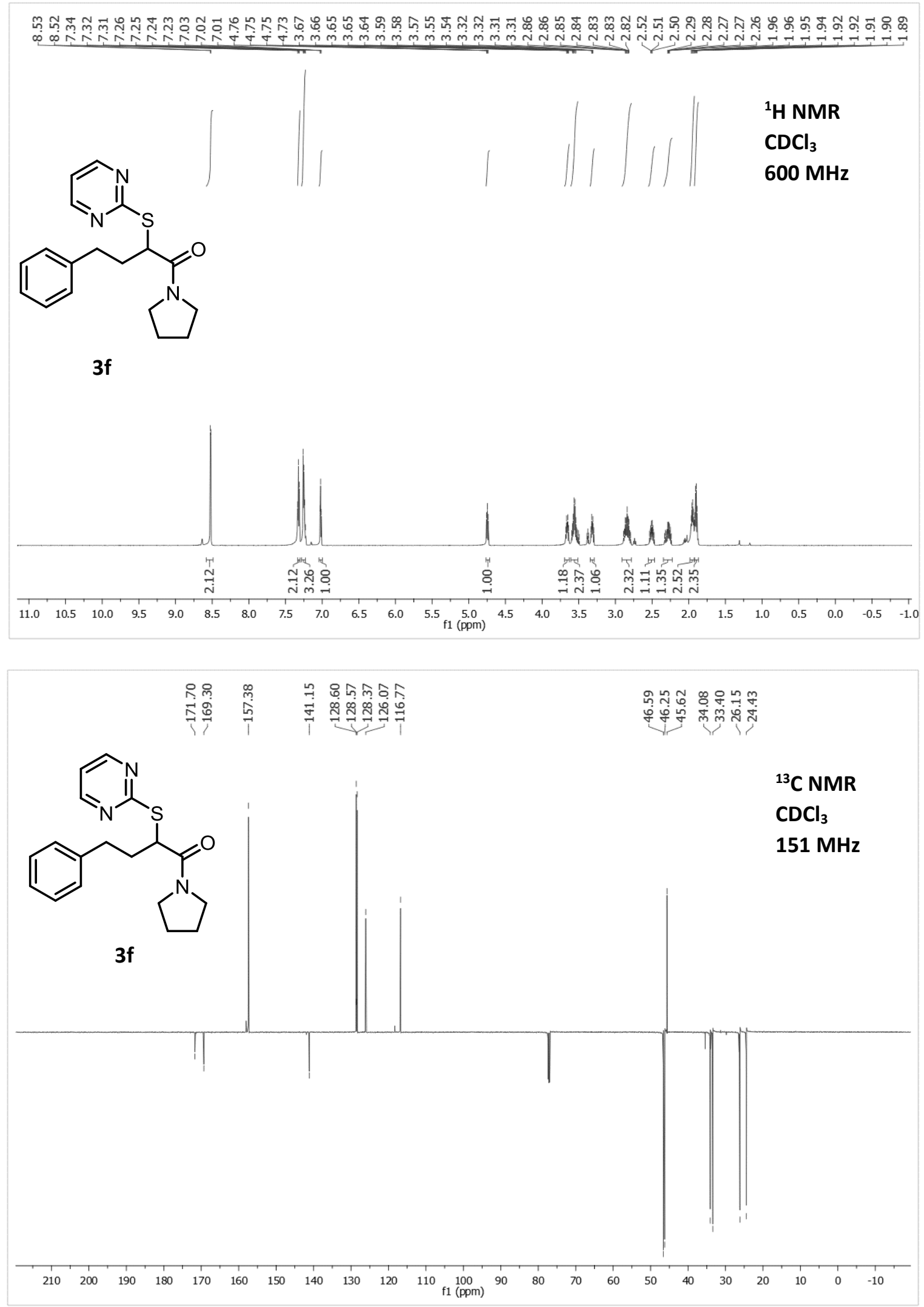




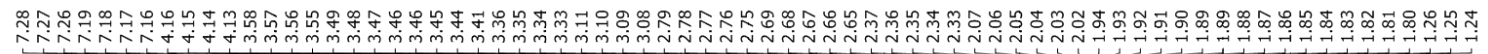

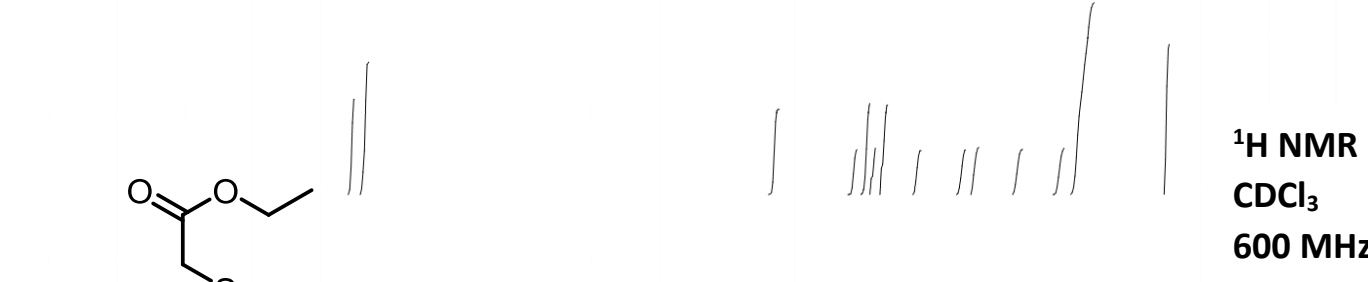<smiles>CSC(CCc1ccccc1)C(=O)N1CCCC1</smiles>

$600 \mathrm{MHz}$

3g
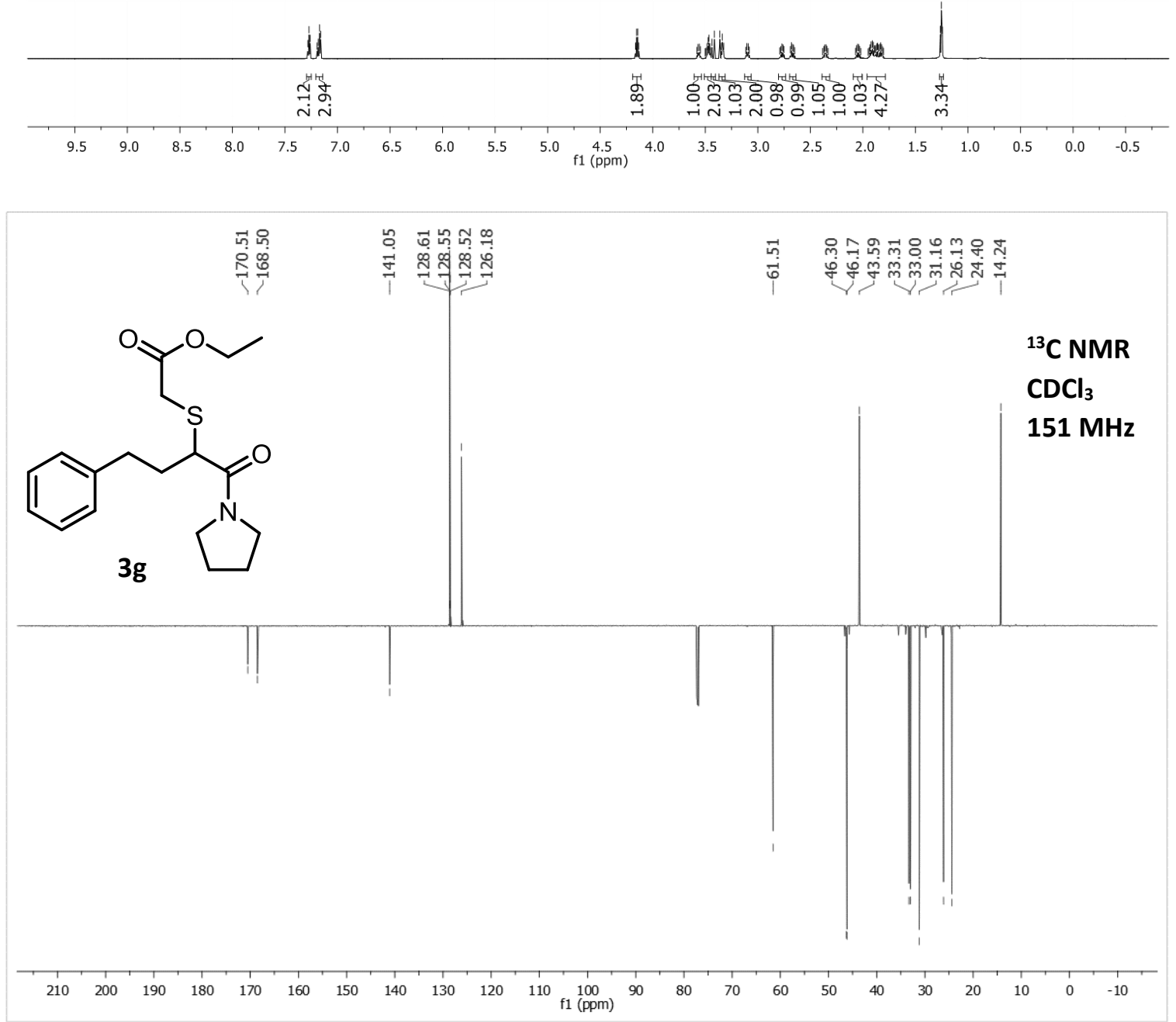


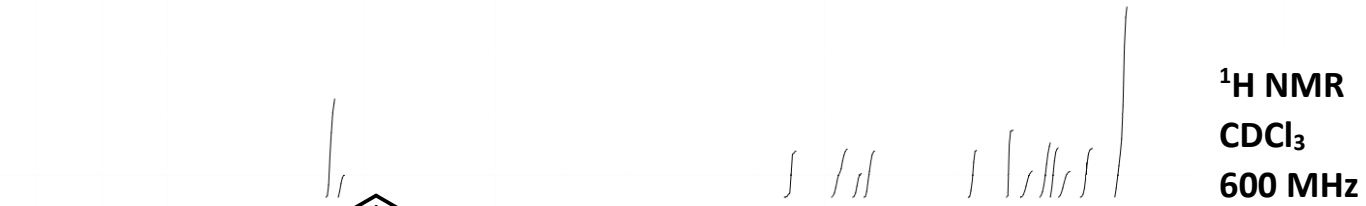<smiles>CC(=O)CCCCCCCC(SCc1ccccc1)C(=O)N1CCCC1</smiles>

3i
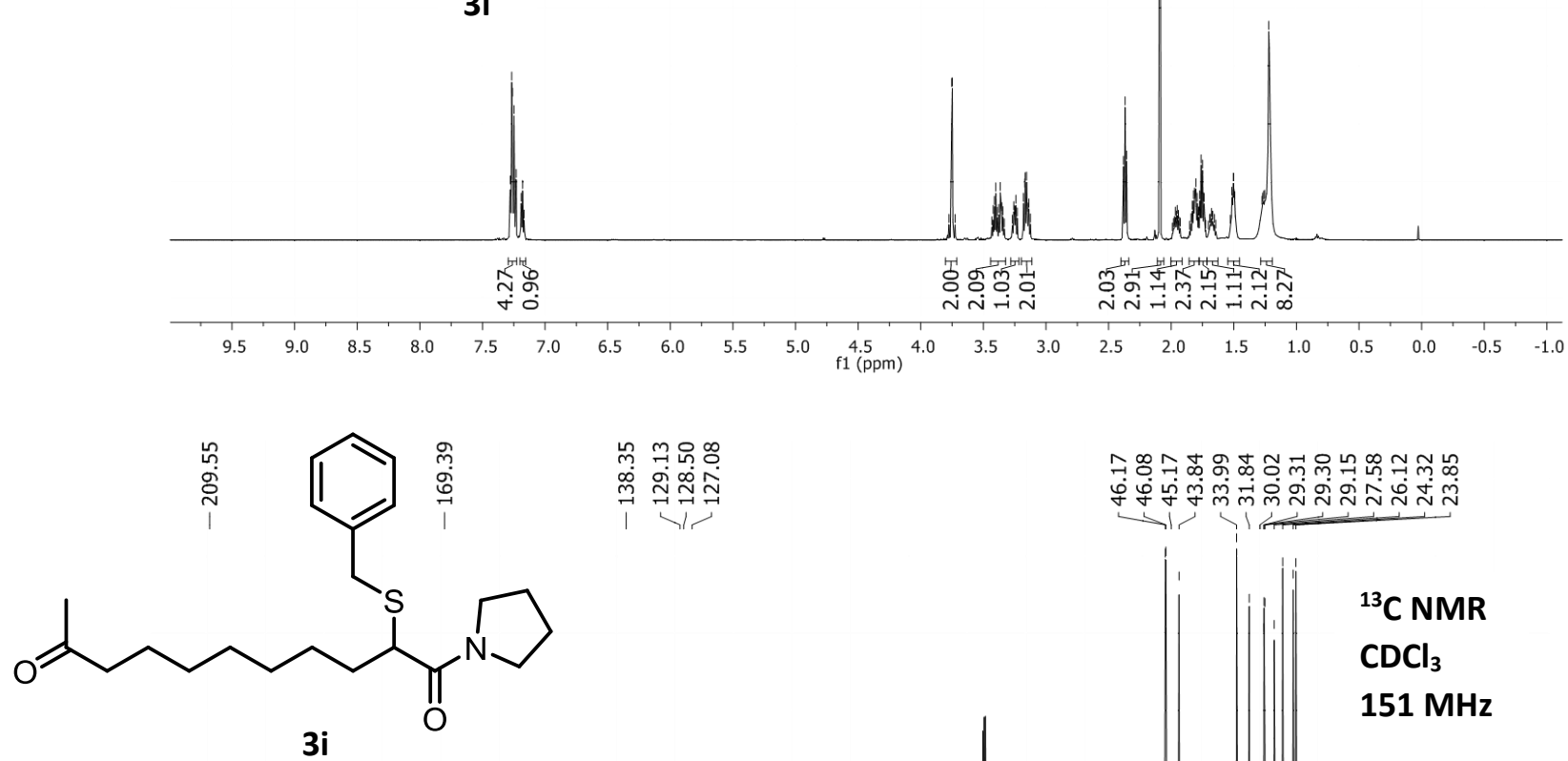

ᄀำ ๆ

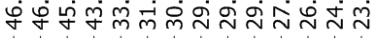

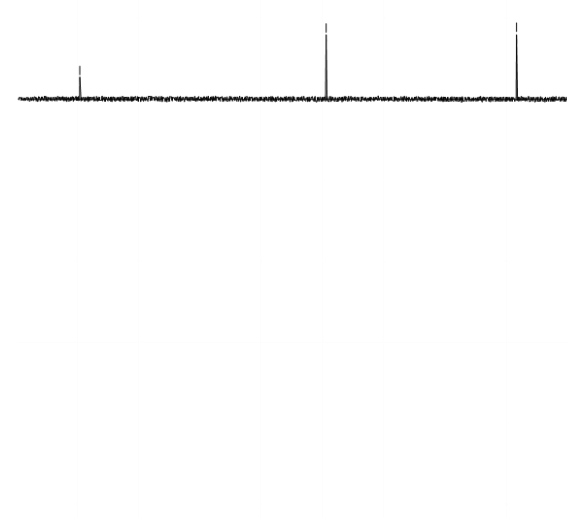

${ }^{13} \mathrm{C}$ NMR

$\mathrm{CDCl}_{3}$

$151 \mathrm{MHz}$

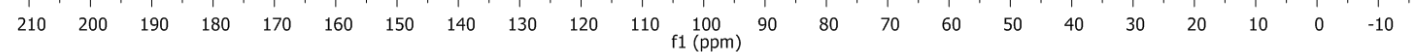



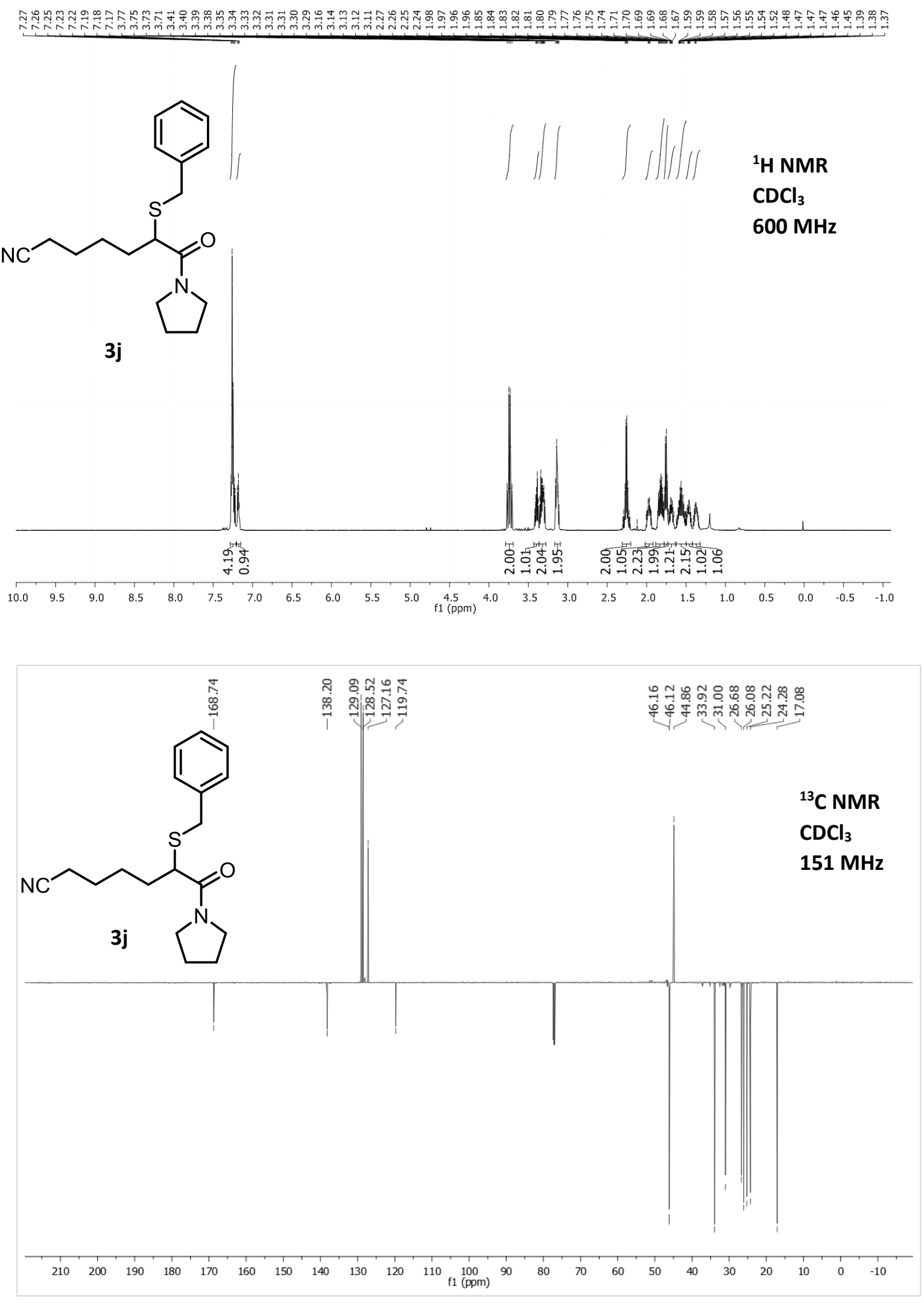

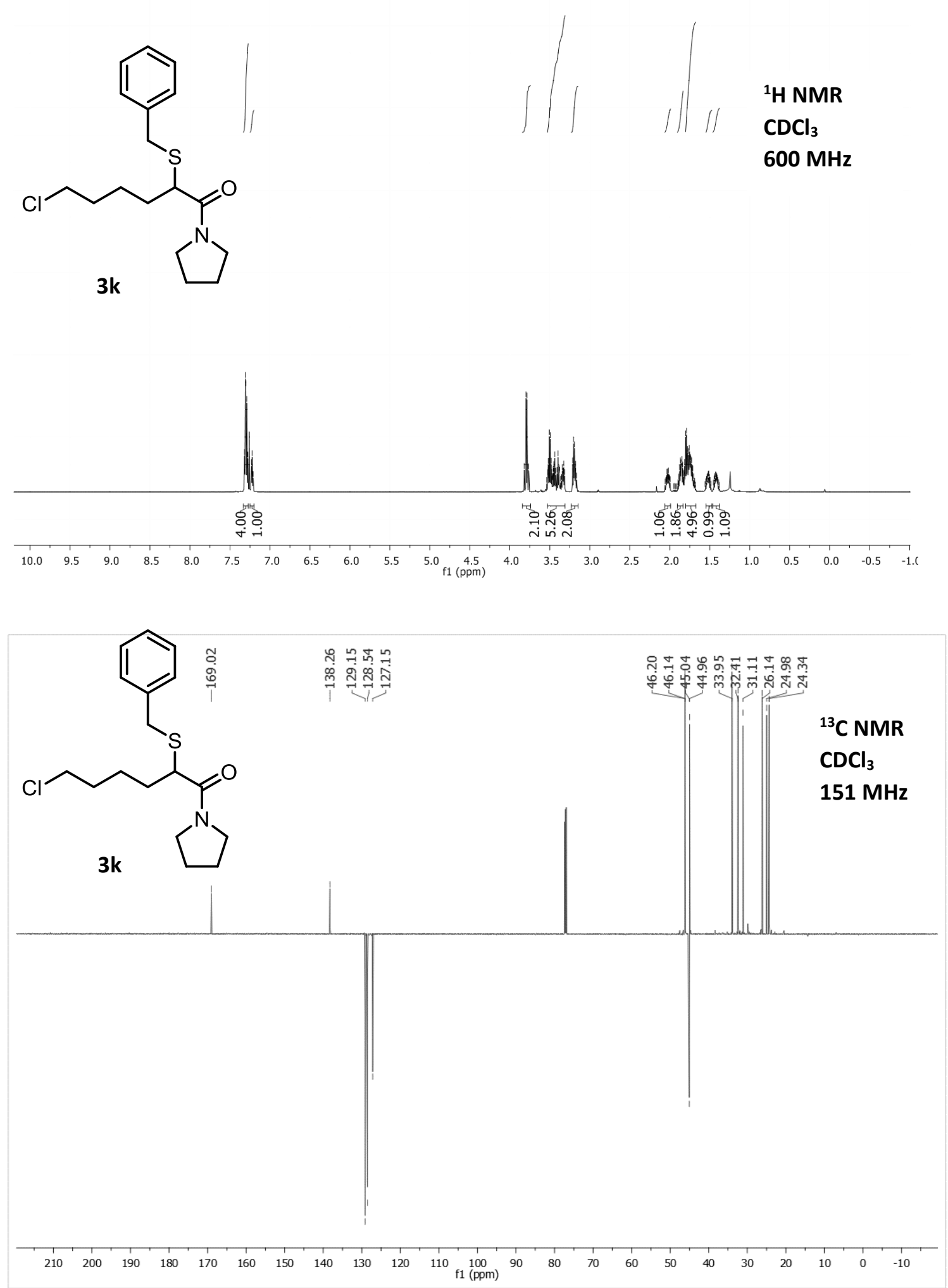

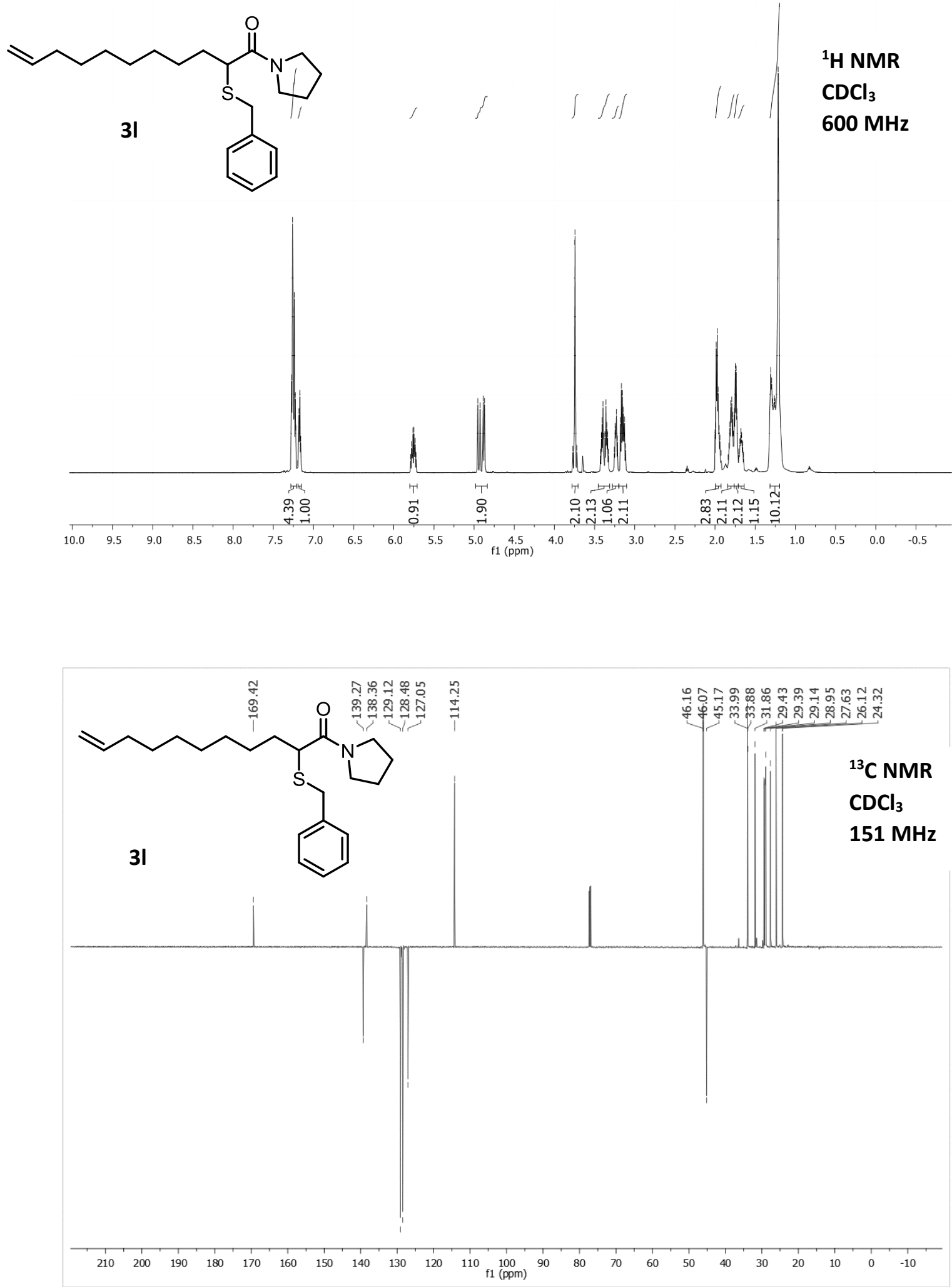

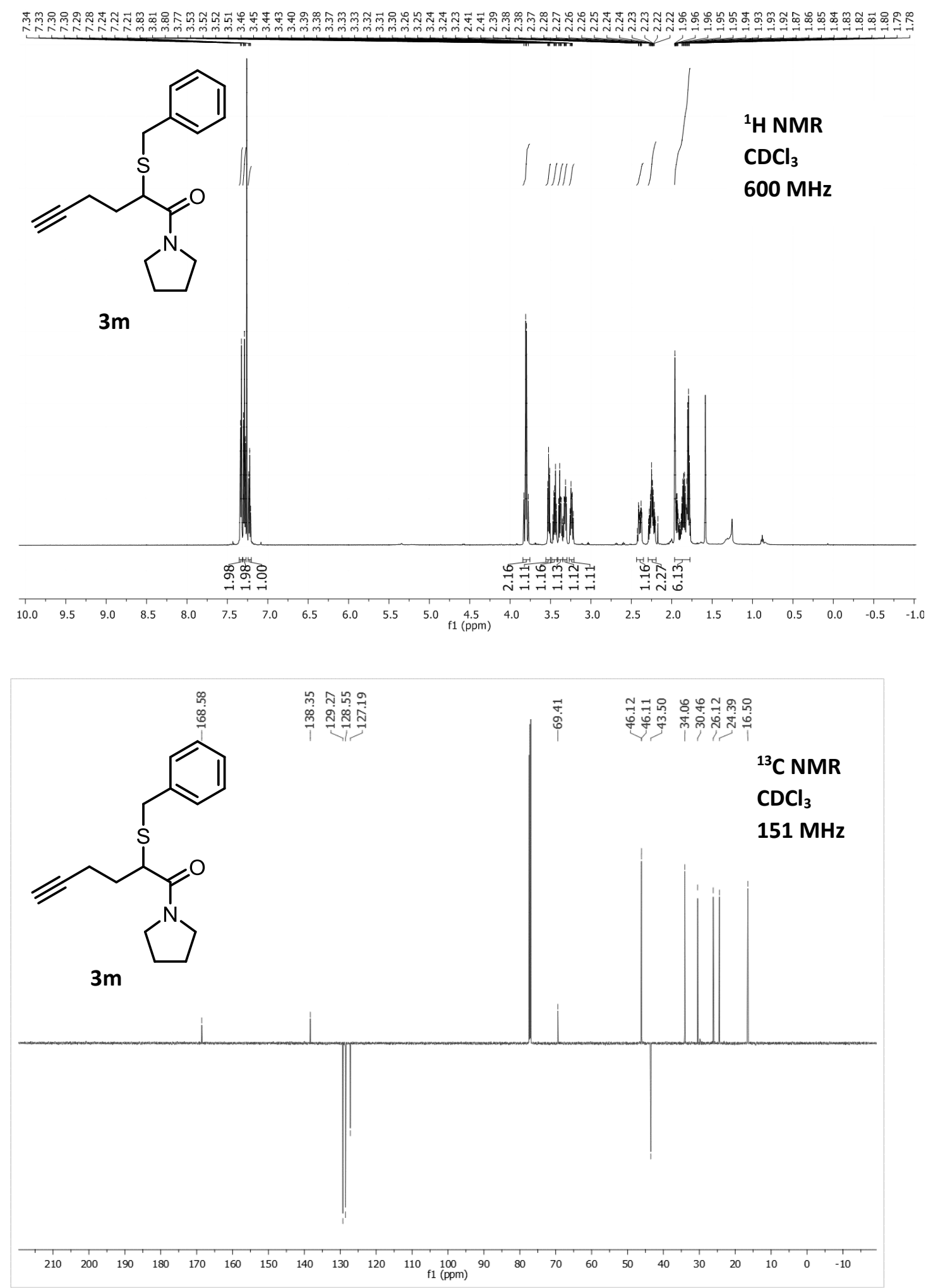

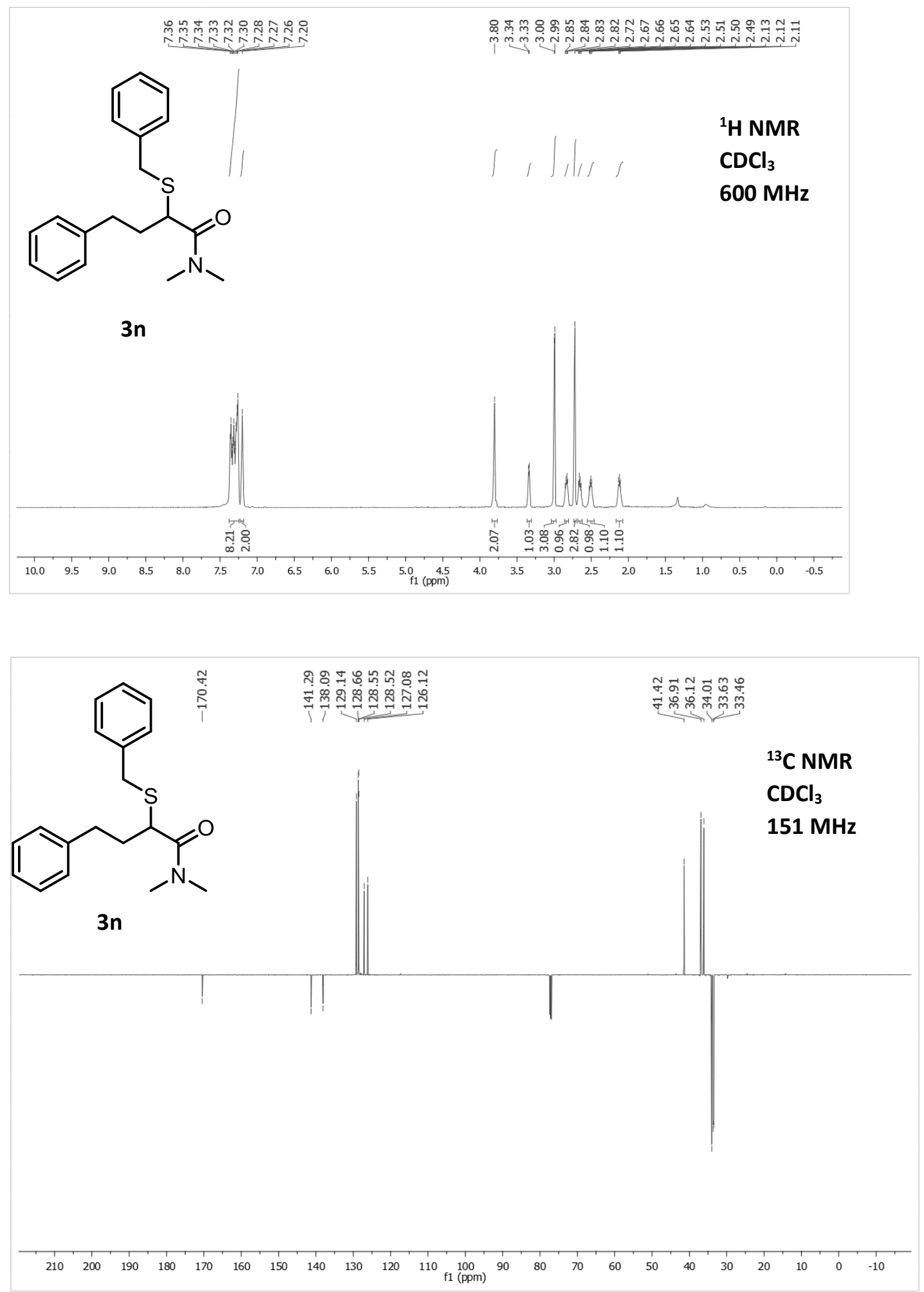

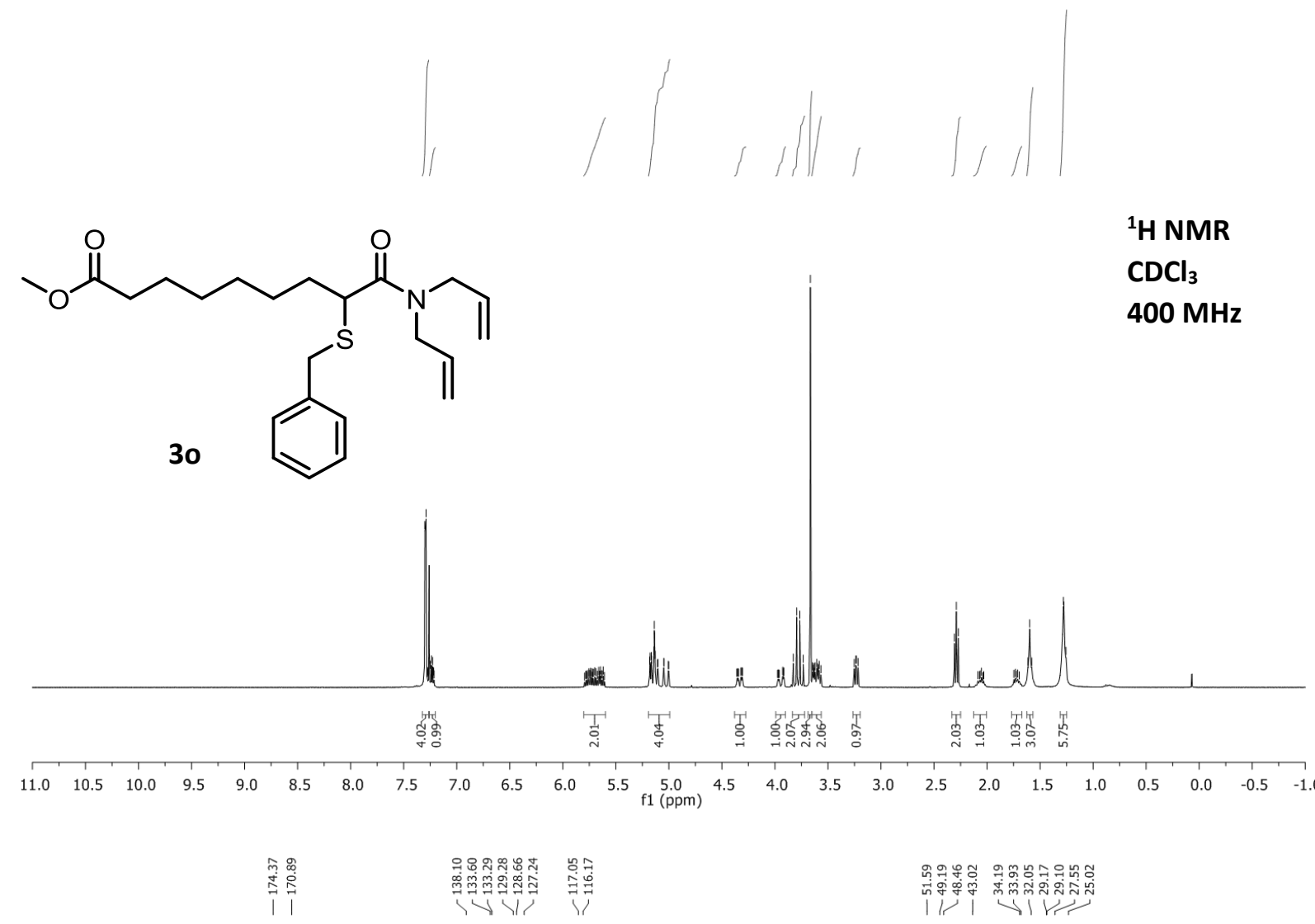

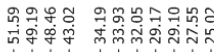

il

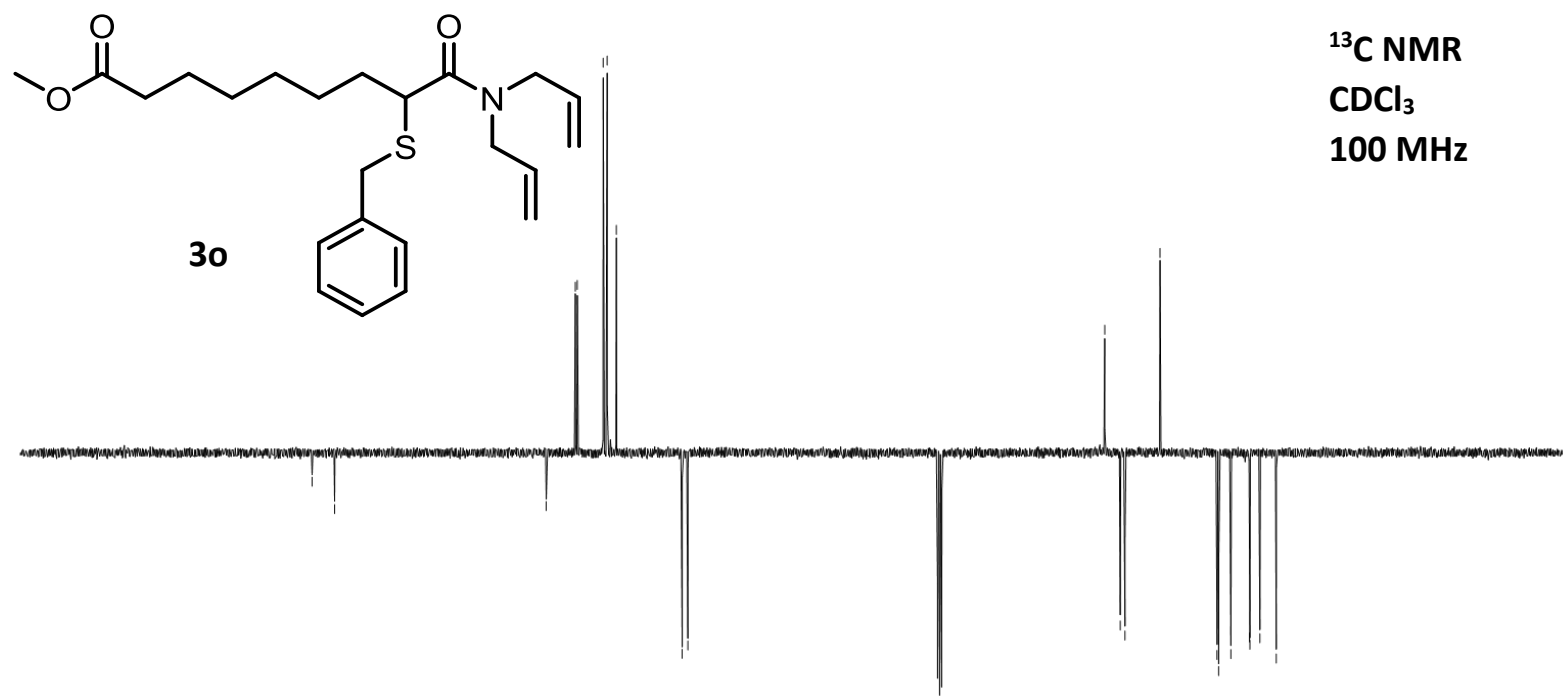

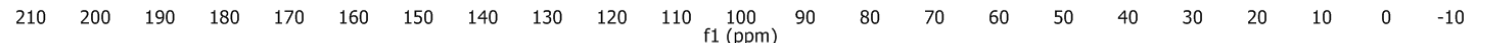



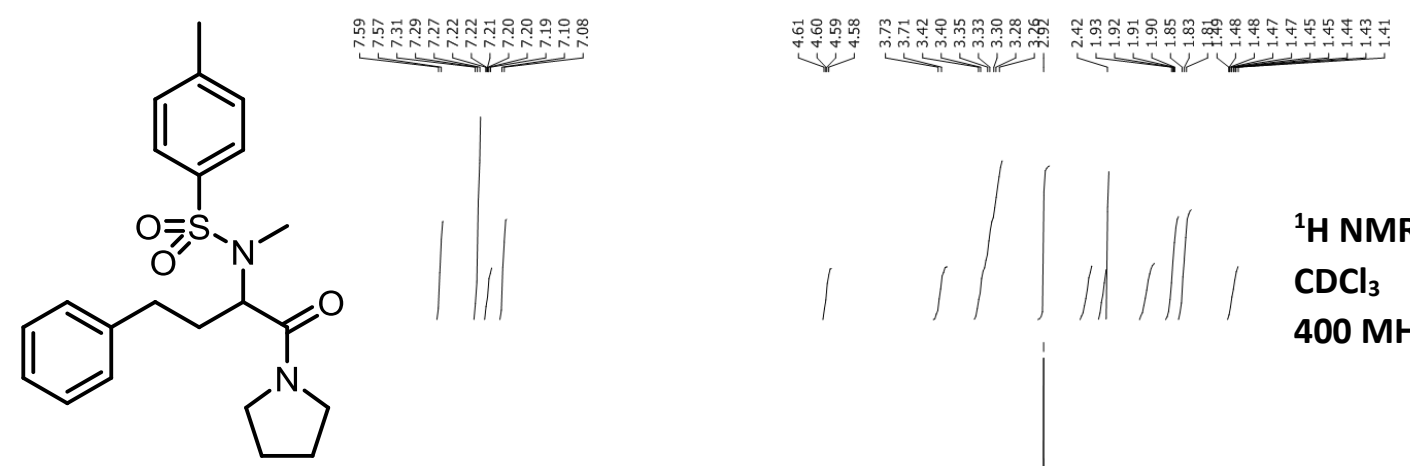

$4 a$
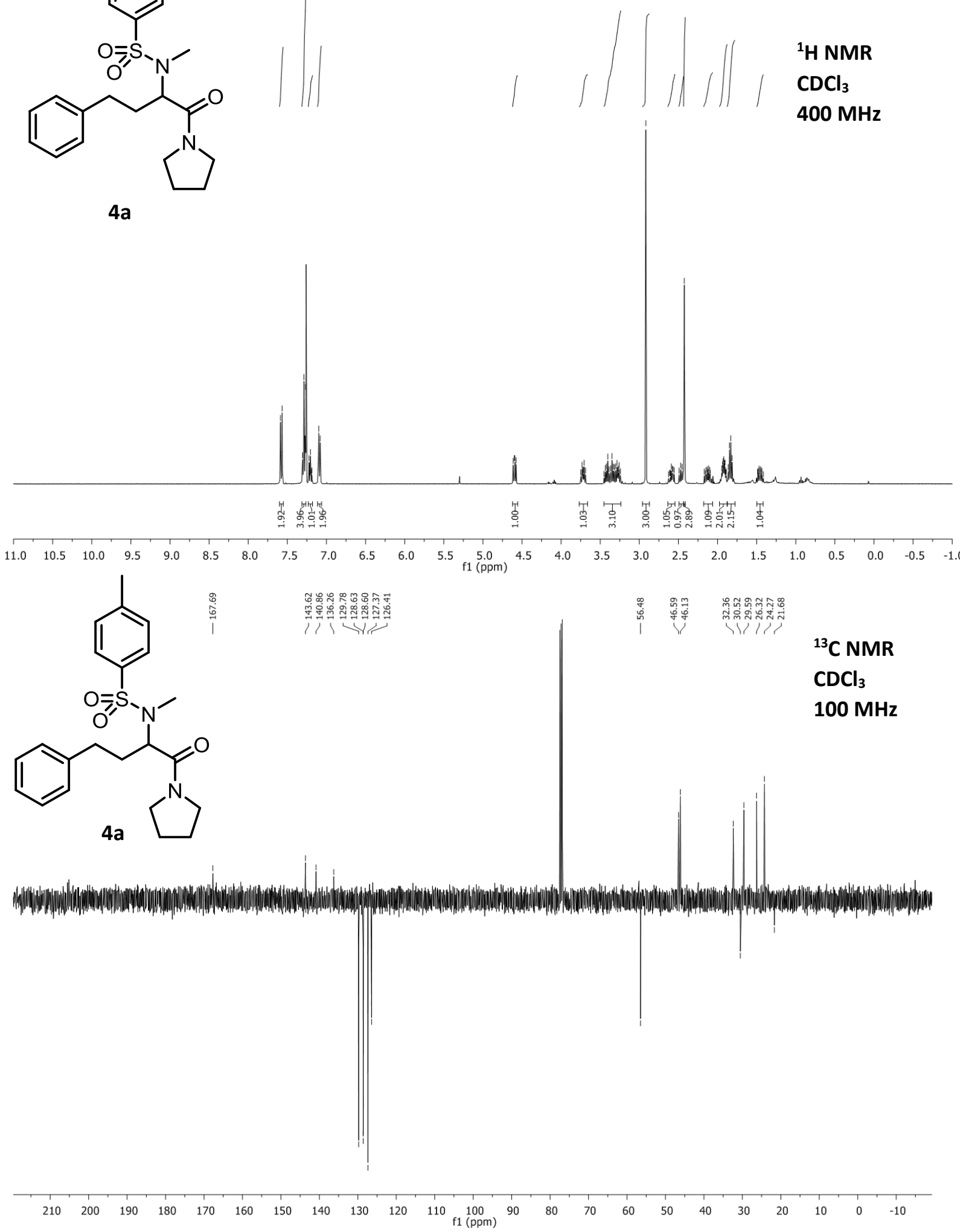

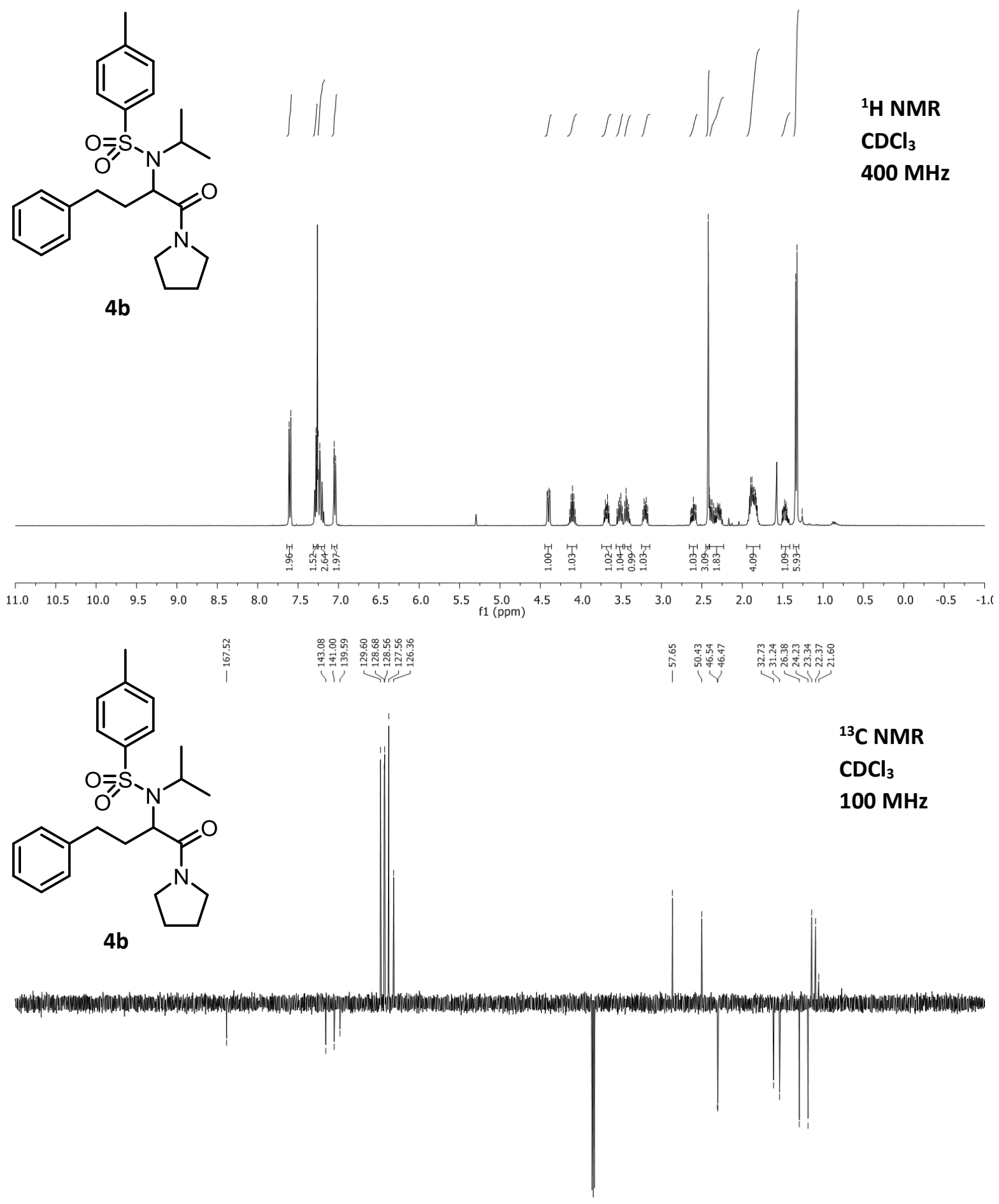

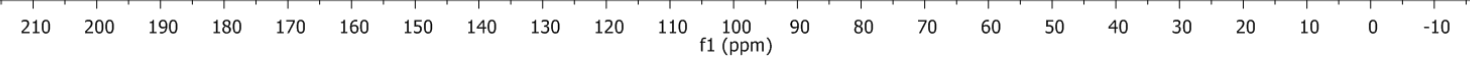




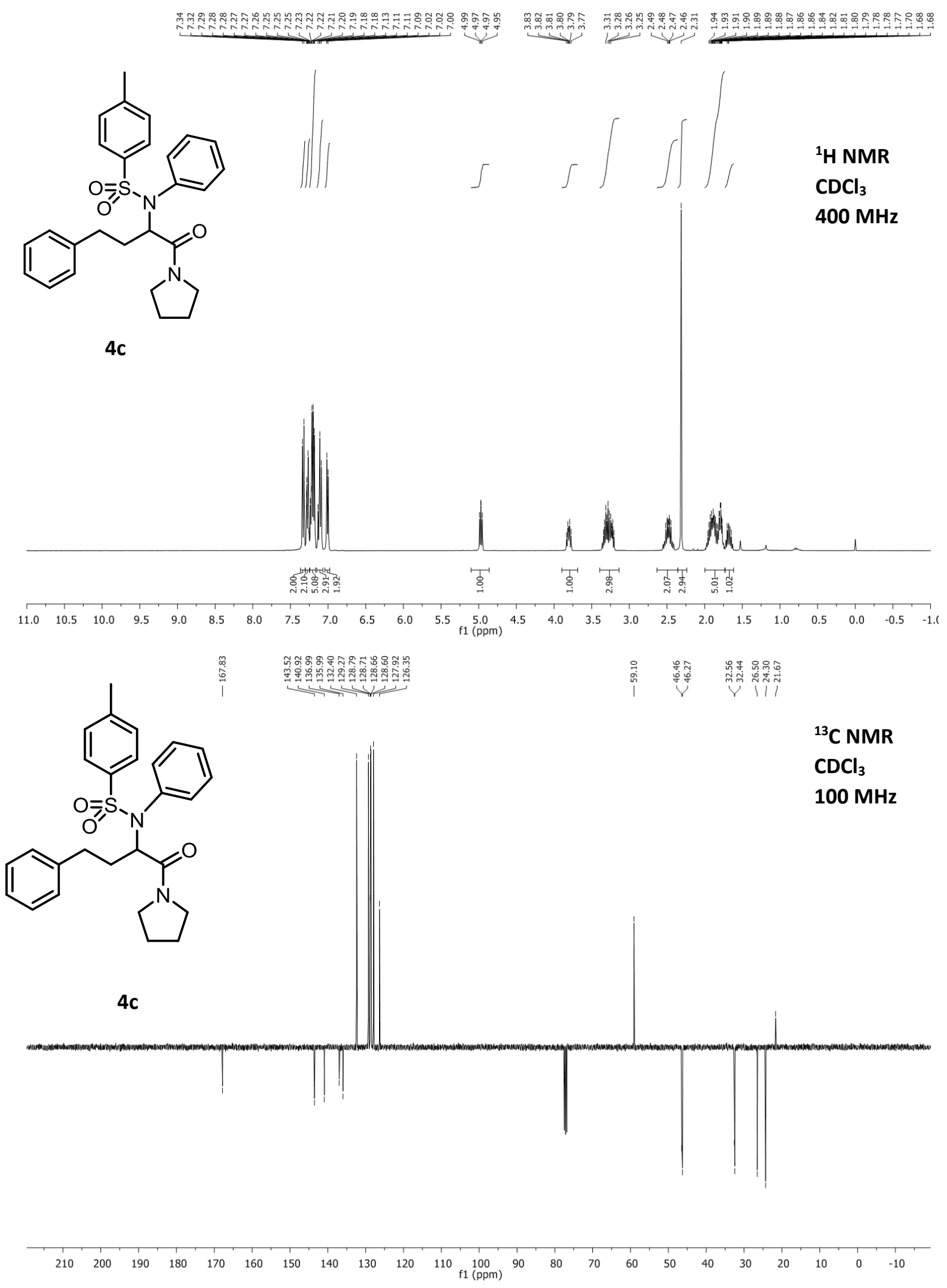


<smiles>COc1ccc(N(C(CCc2ccccc2)C(=O)N2CCCC2)S(=O)(=O)c2ccc(C)cc2)cc1</smiles>

4d

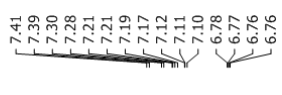

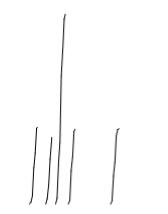

$\sqrt[\substack{0 \\ \sin }]{\sin }$
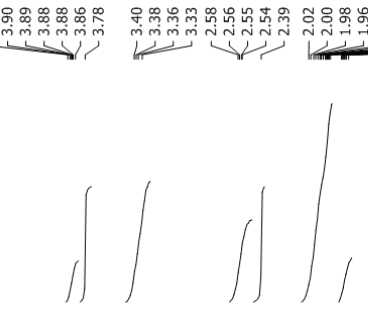

${ }^{1} \mathrm{H}$ NMR

$\mathrm{CDCl}_{3}$

$400 \mathrm{MHz}$
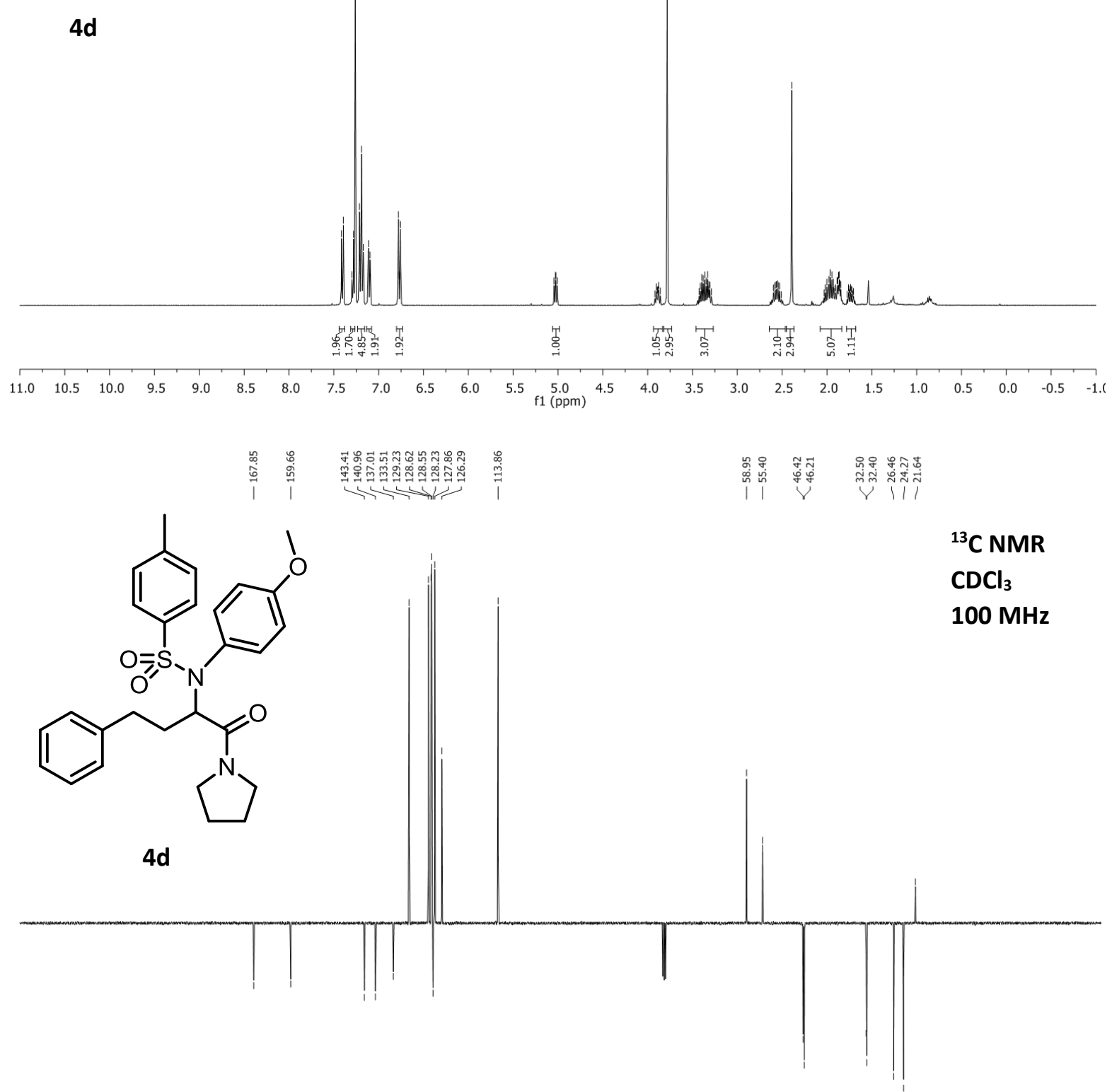

$\begin{array}{lllllllllllllllllllllllllllllllll}210 & 200 & 190 & 180 & 170 & 160 & 150 & 140 & 130 & 120 & 110 & 100 & 90 & 80 & 70 & 60 & 50 & 40 & 30 & 20 & 10 & 0 & -10\end{array}$ 

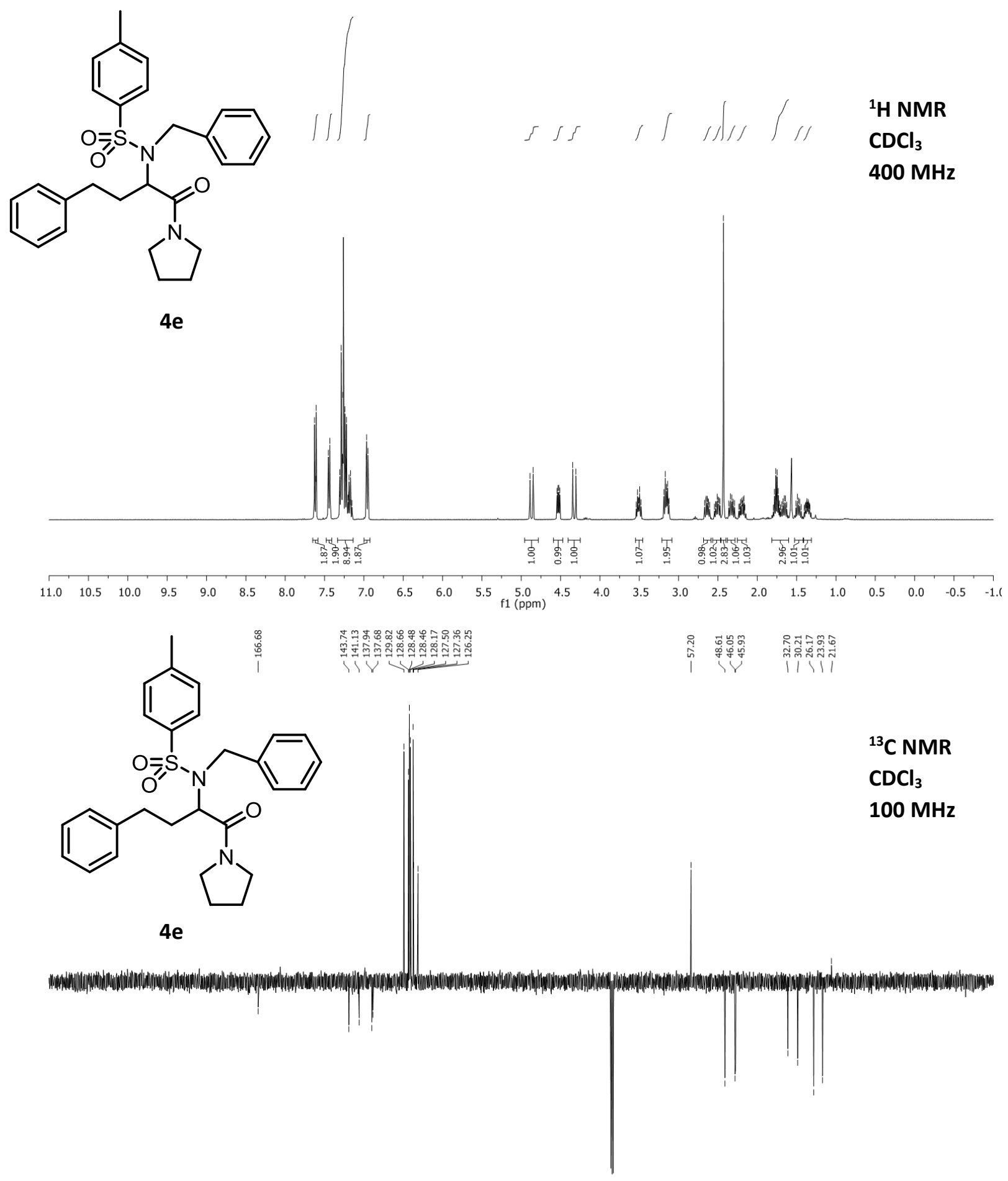

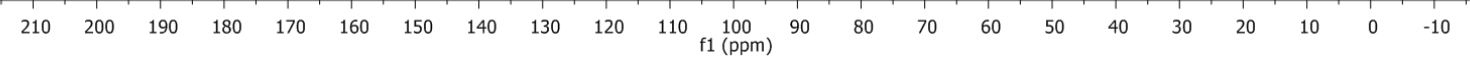



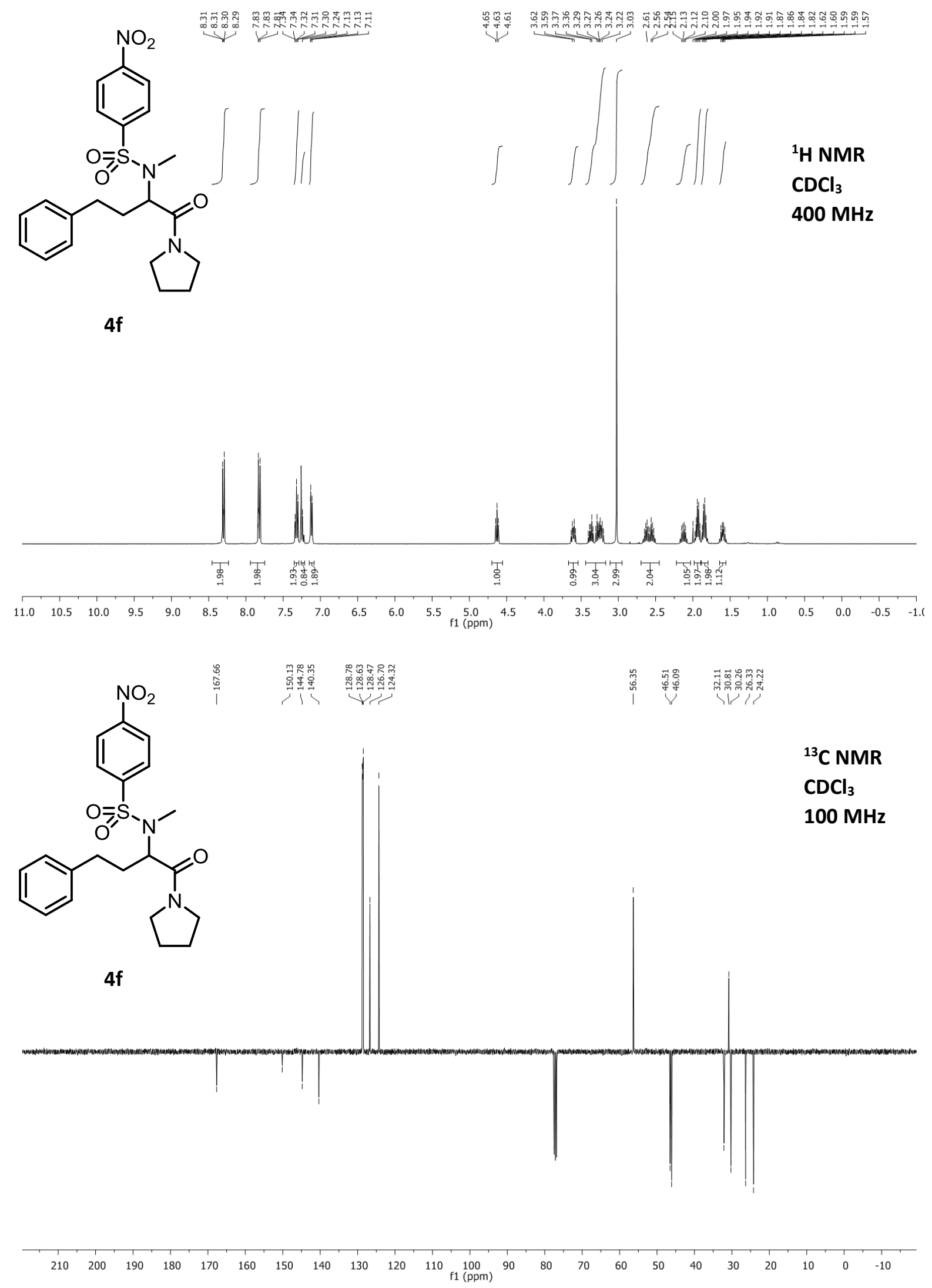


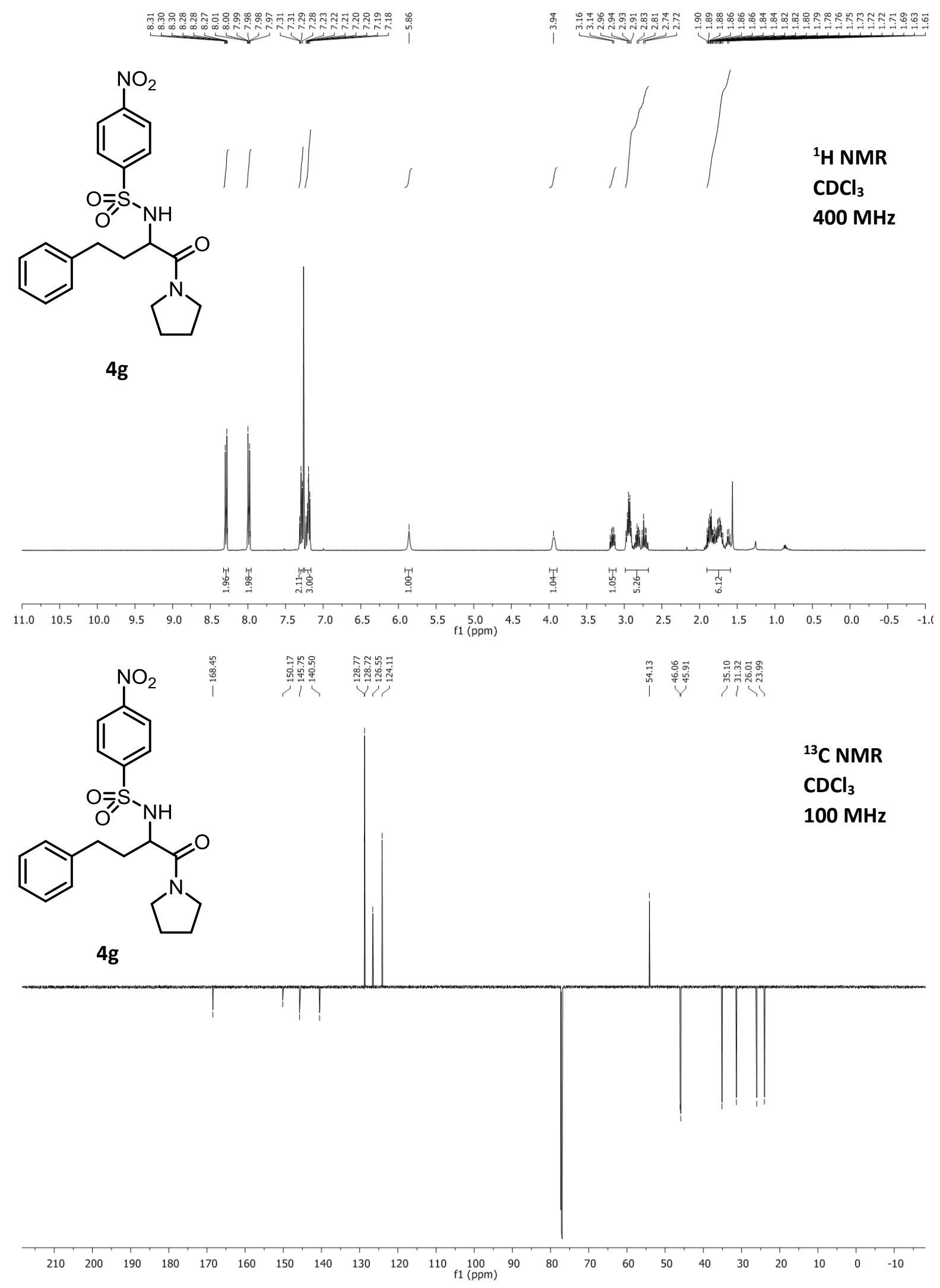



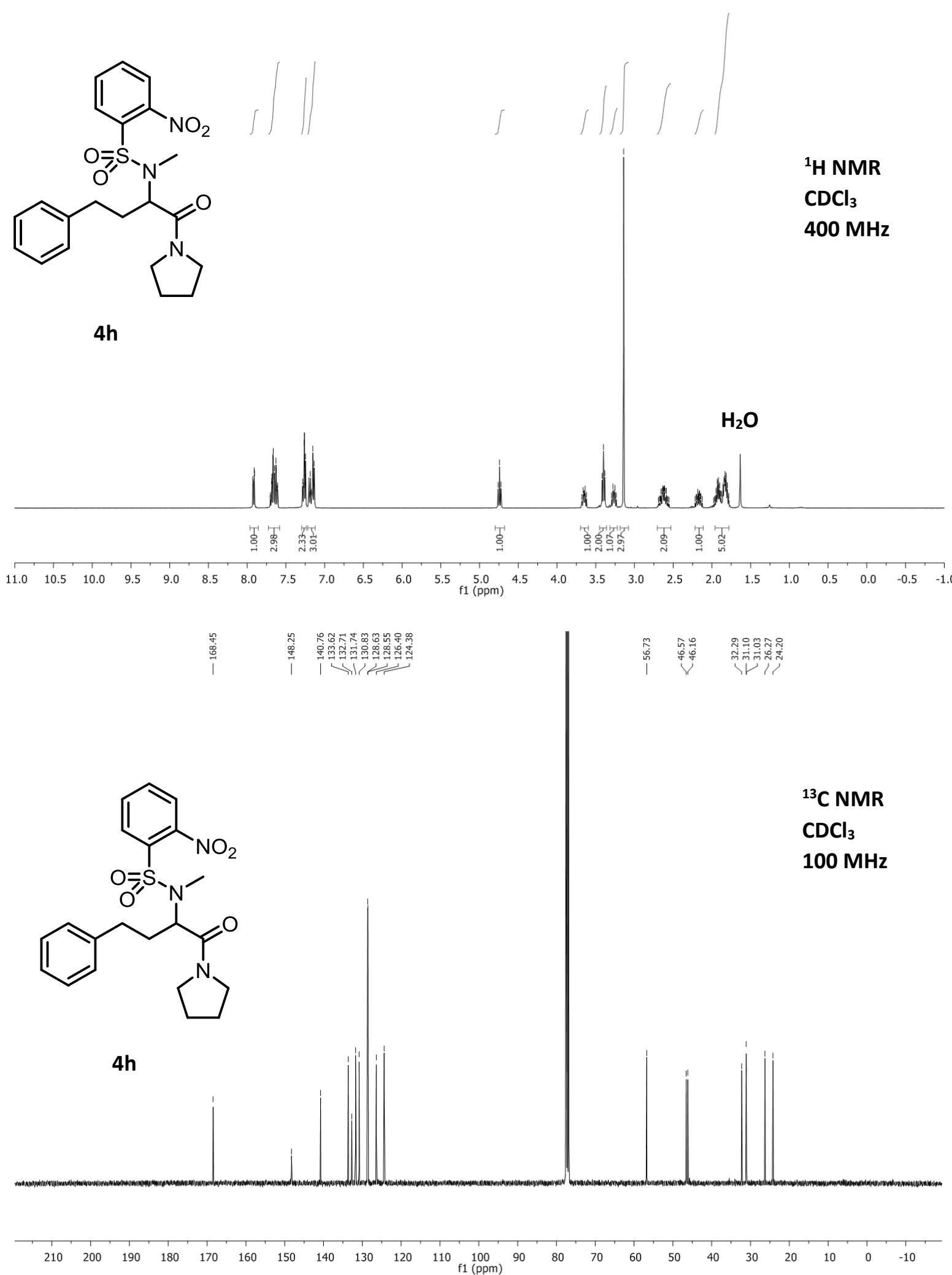
<smiles>CN(C(CCc1ccccc1)C(=O)N1CCCC1)S(=O)(=O)c1ccccn1</smiles>

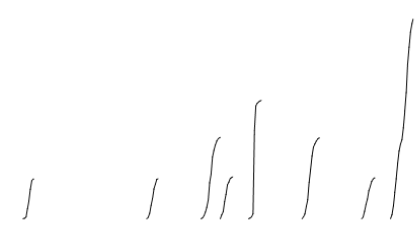

${ }^{1} \mathrm{H}$ NMR

$\mathrm{CDCl}_{3}$ $400 \mathrm{MHz}$

$4 \mathbf{i}$
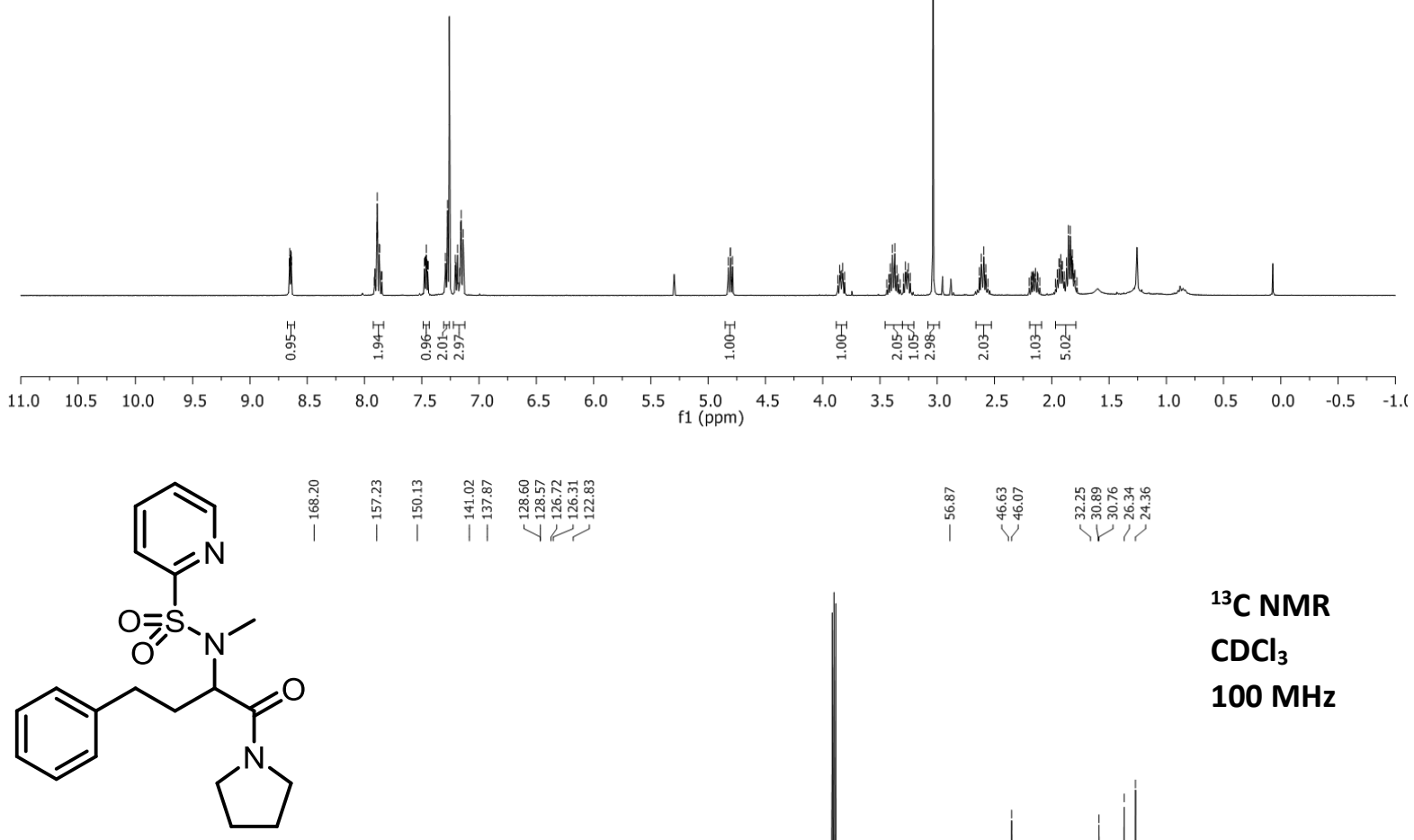

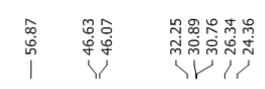

$4 \mathbf{i}$

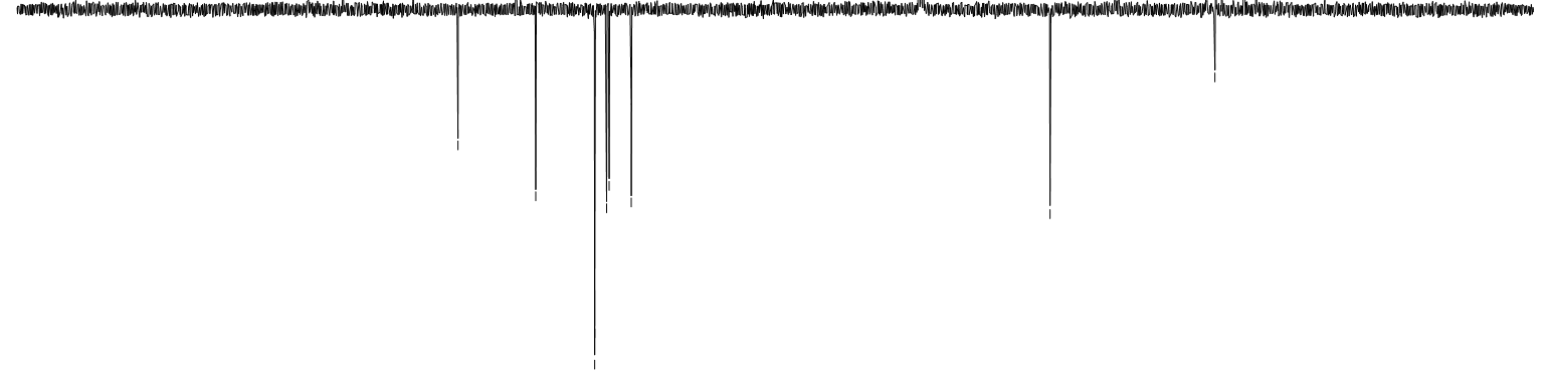

$\begin{array}{lllllllllllllllllllllllllll}210 & 200 & 190 & 180 & 170 & 160 & 150 & 140 & 130 & 120 & 110 & 100 & 90 & 80 & 70 & 60 & 50 & 40 & 30 & 20 & 10 & 0 & -10\end{array}$ 

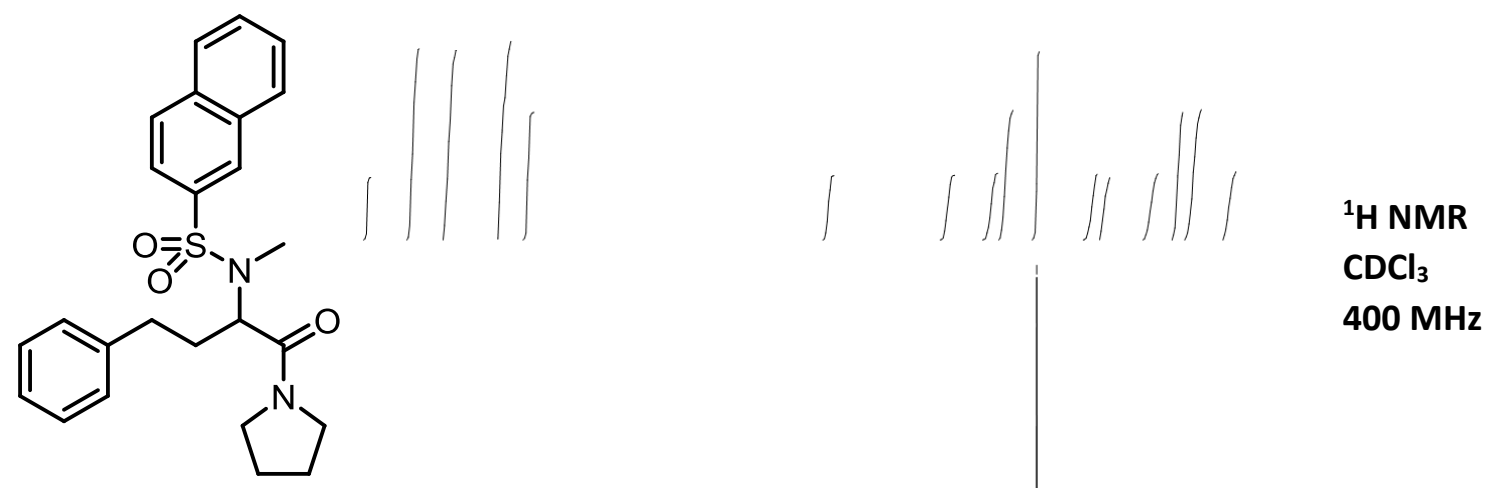

4j
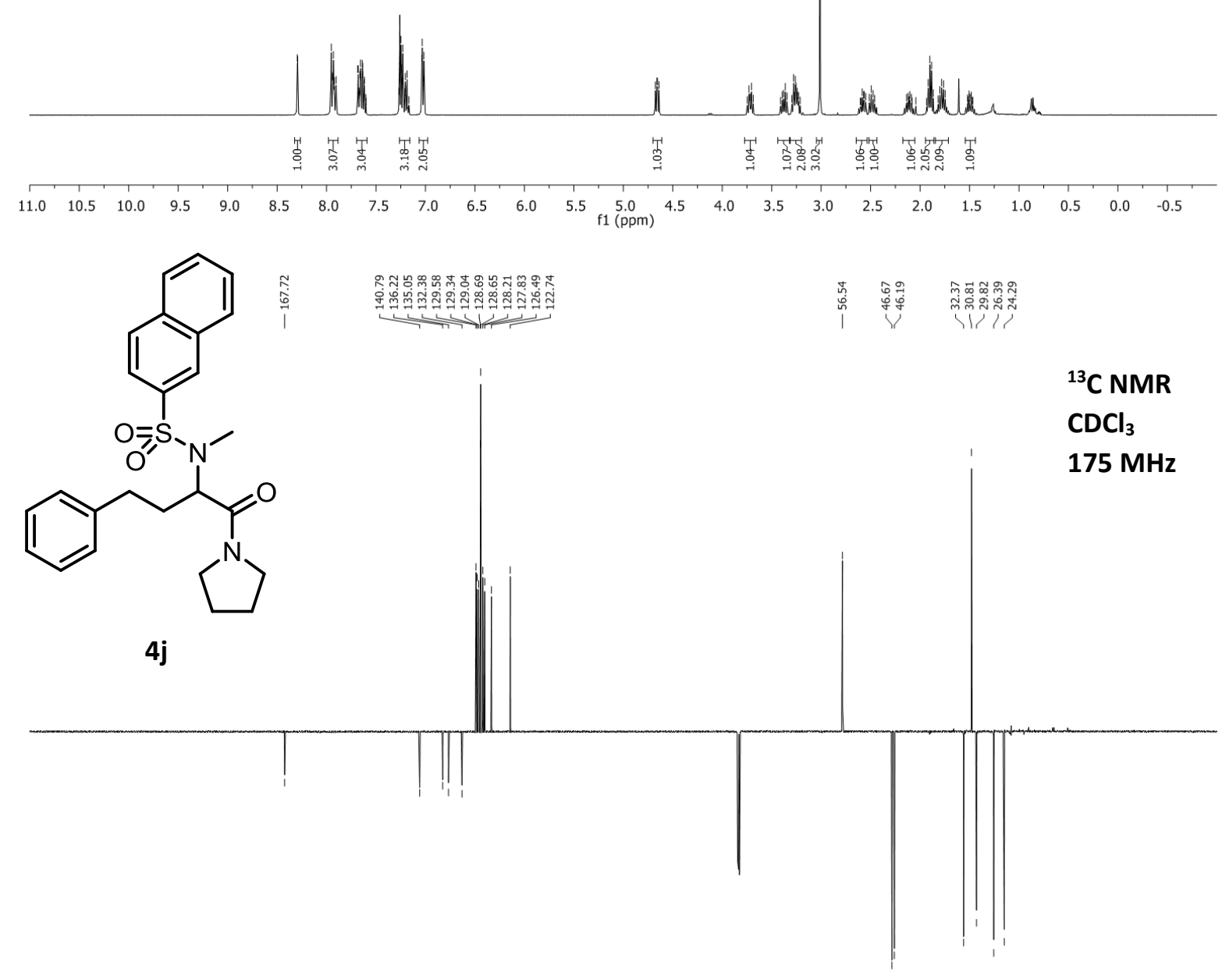

$\begin{array}{llllllllllllllllllllllllll}210 & 200 & 190 & 180 & 170 & 160 & 150 & 140 & 130 & 120 & 110 & 100 & 90 & 80 & 70 & 60 & 50 & 40 & 30 & 20 & 10 & 0 & -10\end{array}$ 

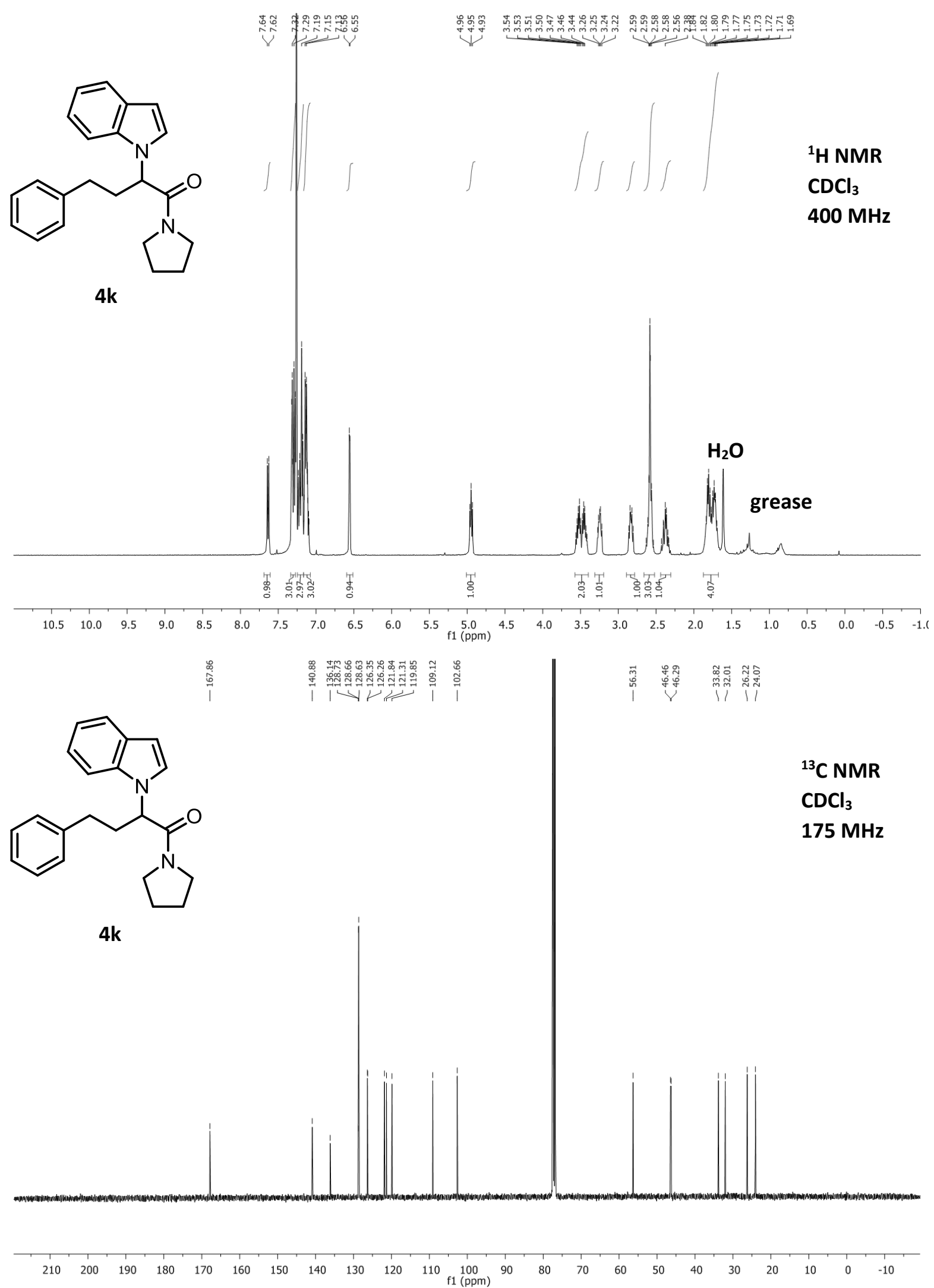


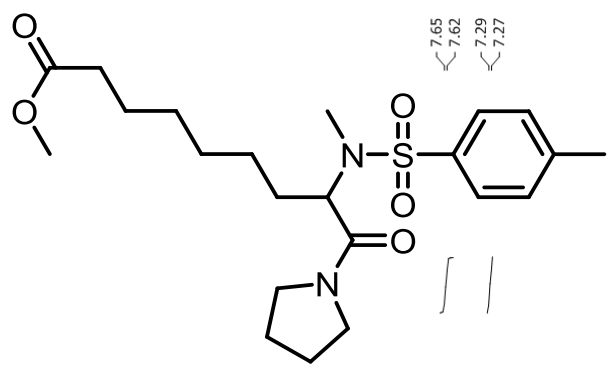

41

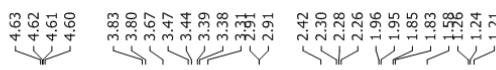

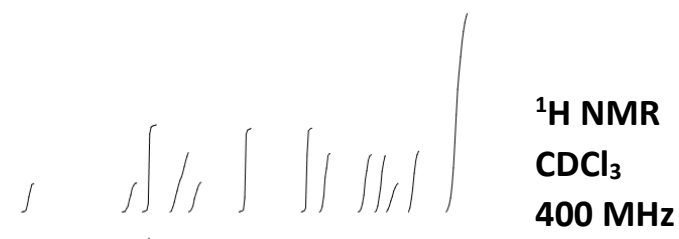

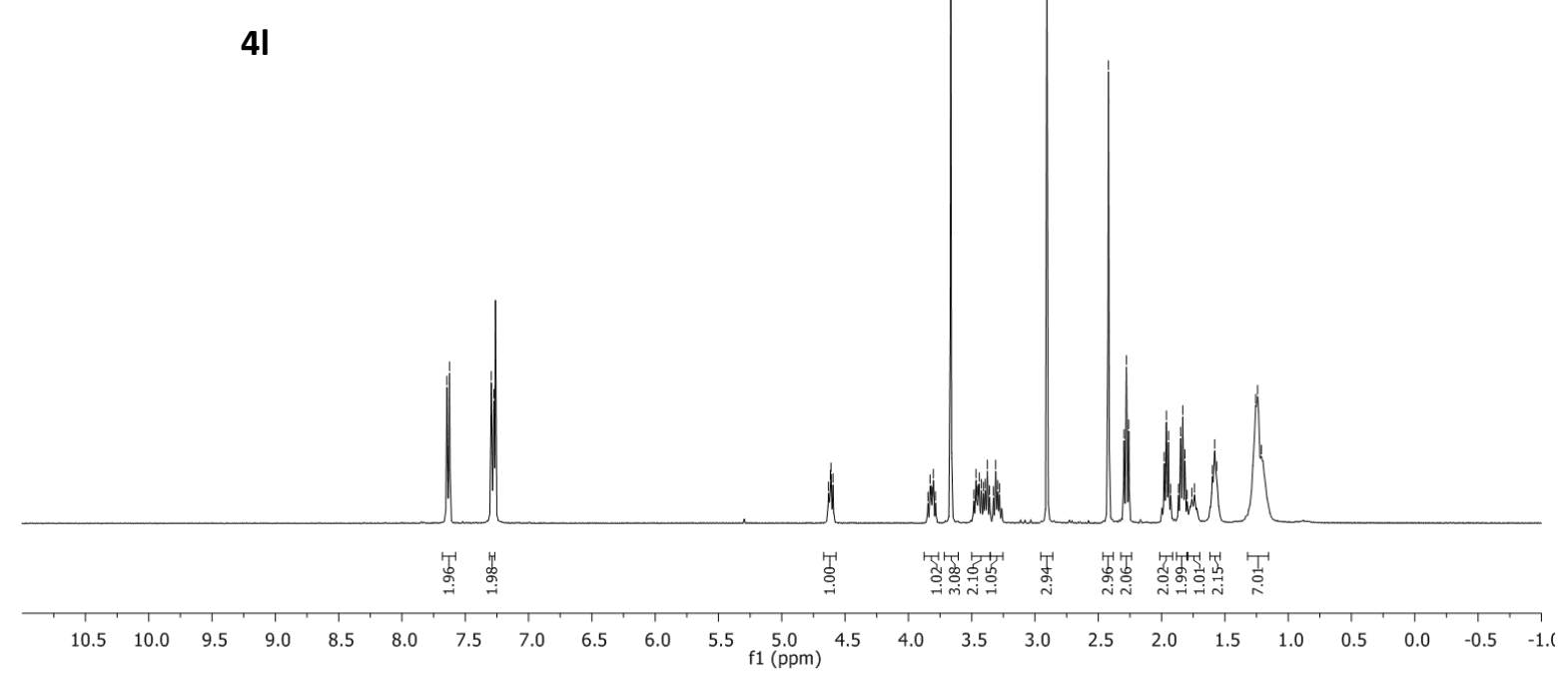

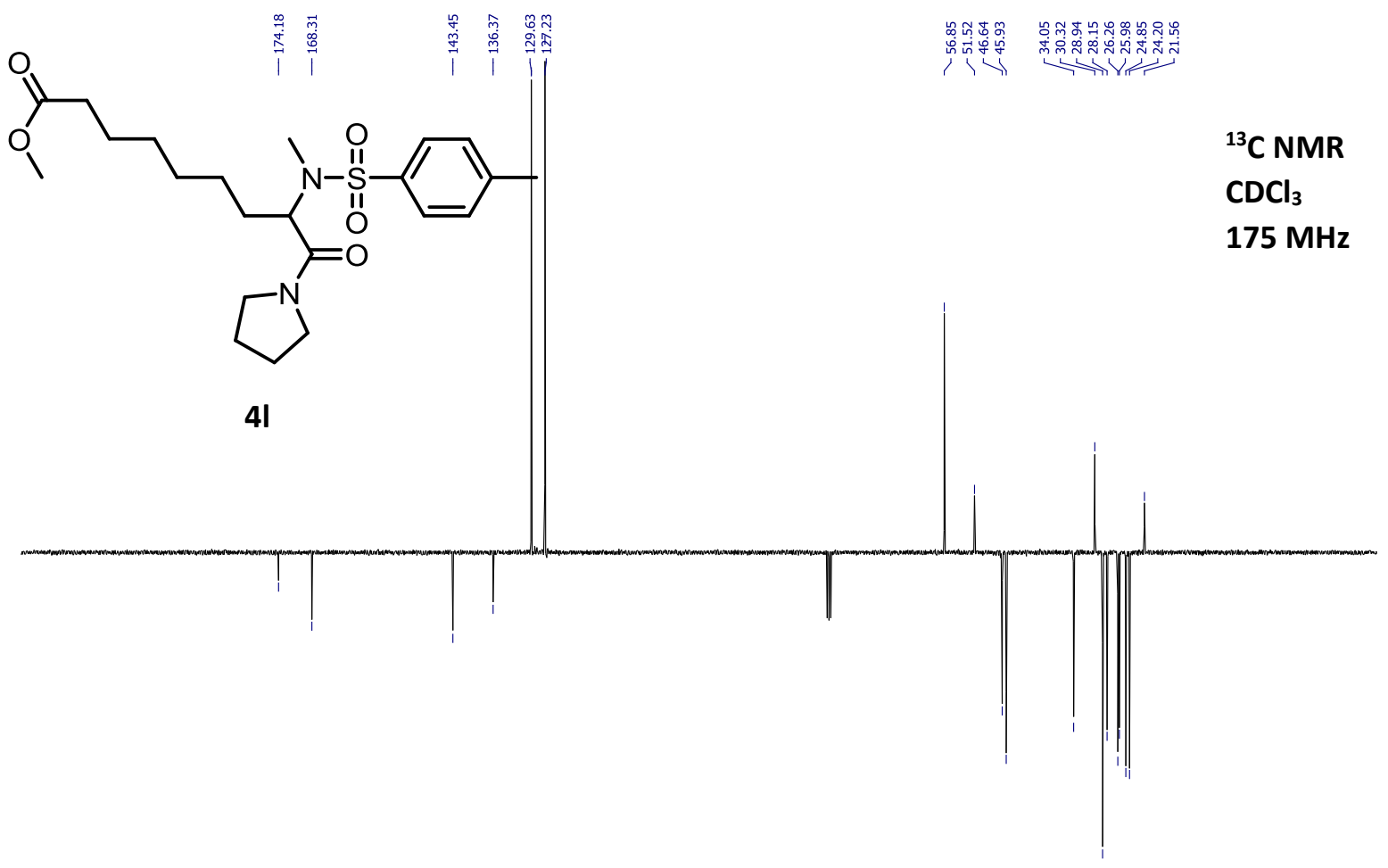

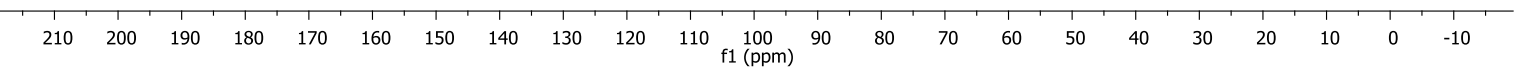



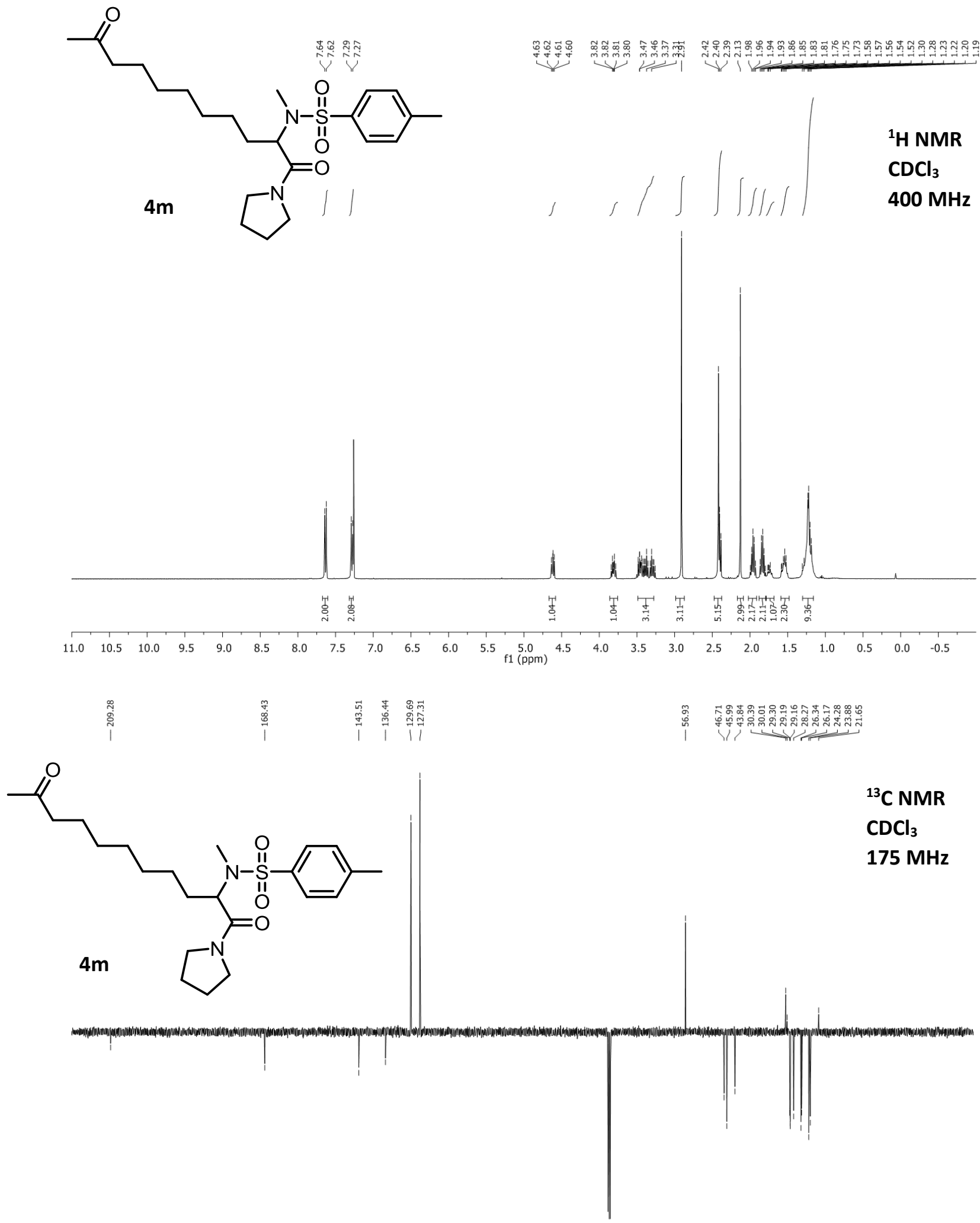

$\begin{array}{llllllllllllllllllllllll}210 & 200 & 190 & 180 & 170 & 160 & 150 & 140 & 130 & 120 & 110 & \underset{\mathrm{f} 1}{1(\mathrm{ppm})} & 90 & 80 & 70 & 60 & 50 & 40 & 30 & 20 & 10 & 0 & -10\end{array}$




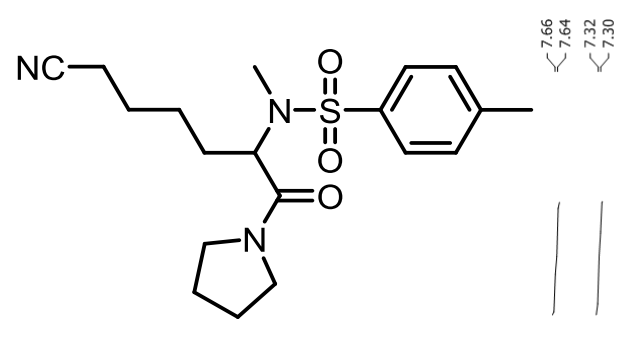

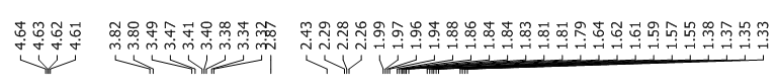
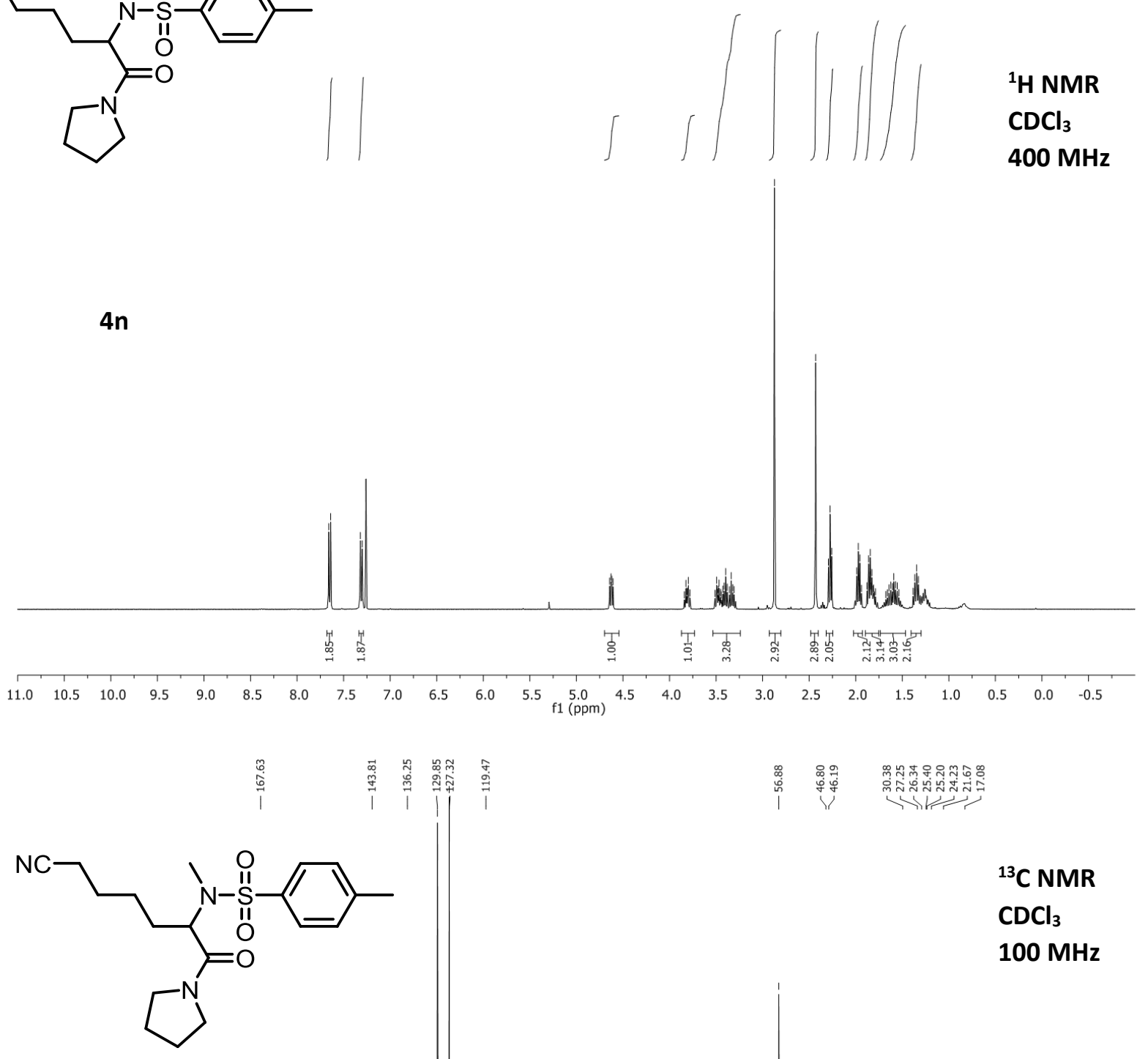

${ }^{13}$ C NMR $\mathrm{CDCl}_{3}$ $100 \mathrm{MHz}$

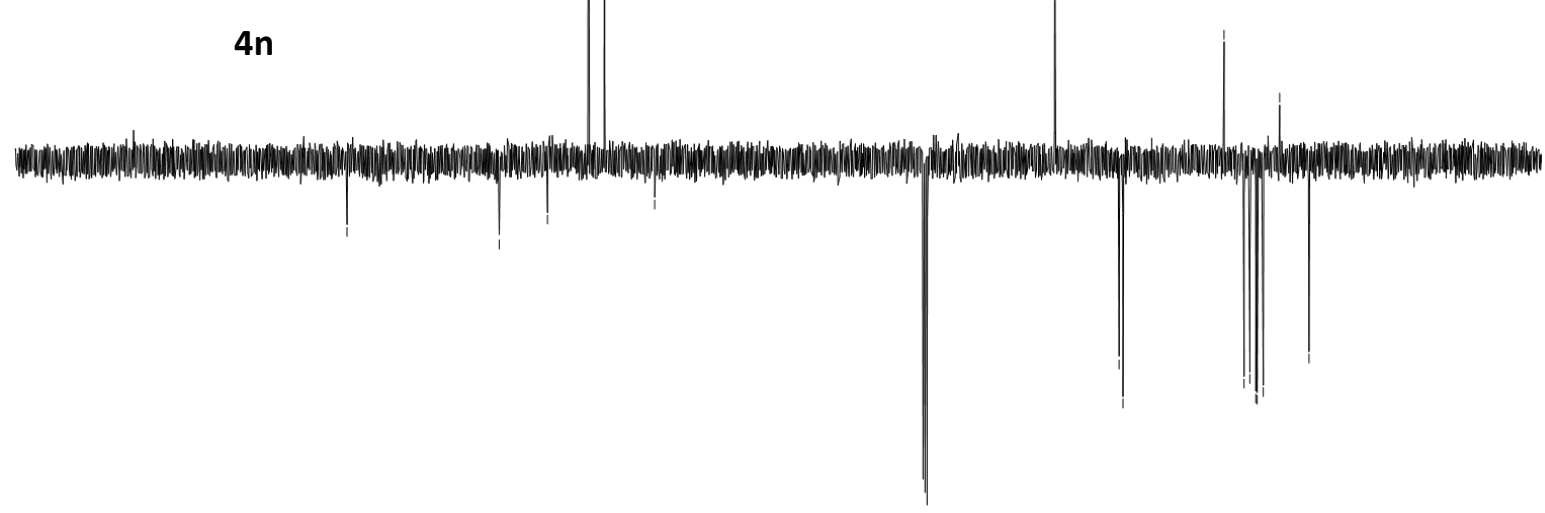

$\begin{array}{lllllllllllllllllllllll}210 & 200 & 190 & 180 & 170 & 160 & 150 & 140 & 130 & 120 & 110 & \begin{array}{c}100 \\ \mathrm{f} 1(\mathrm{ppm})\end{array} & 90 & 80 & 70 & 60 & 50 & 40 & 30 & 20 & 10 & 0 & -10\end{array}$ 

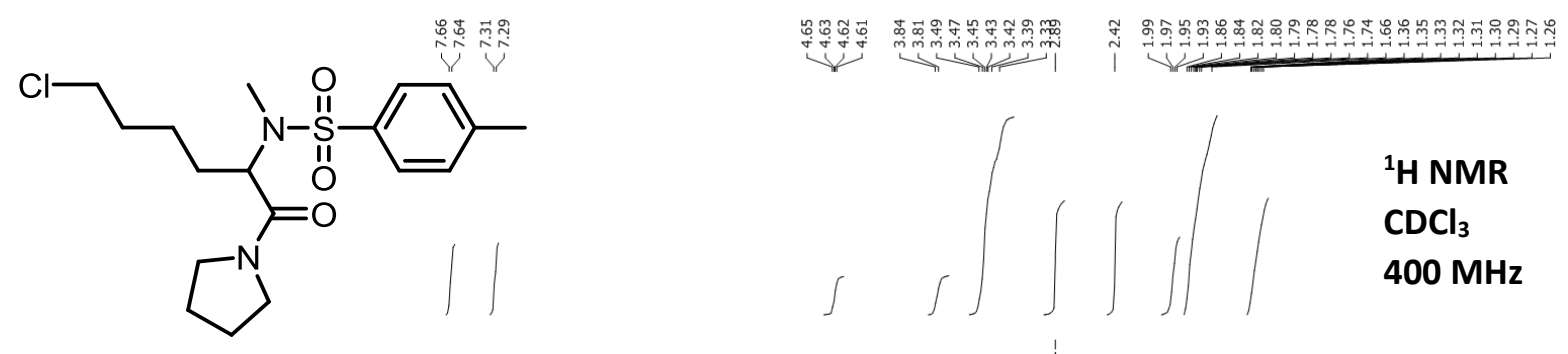

40
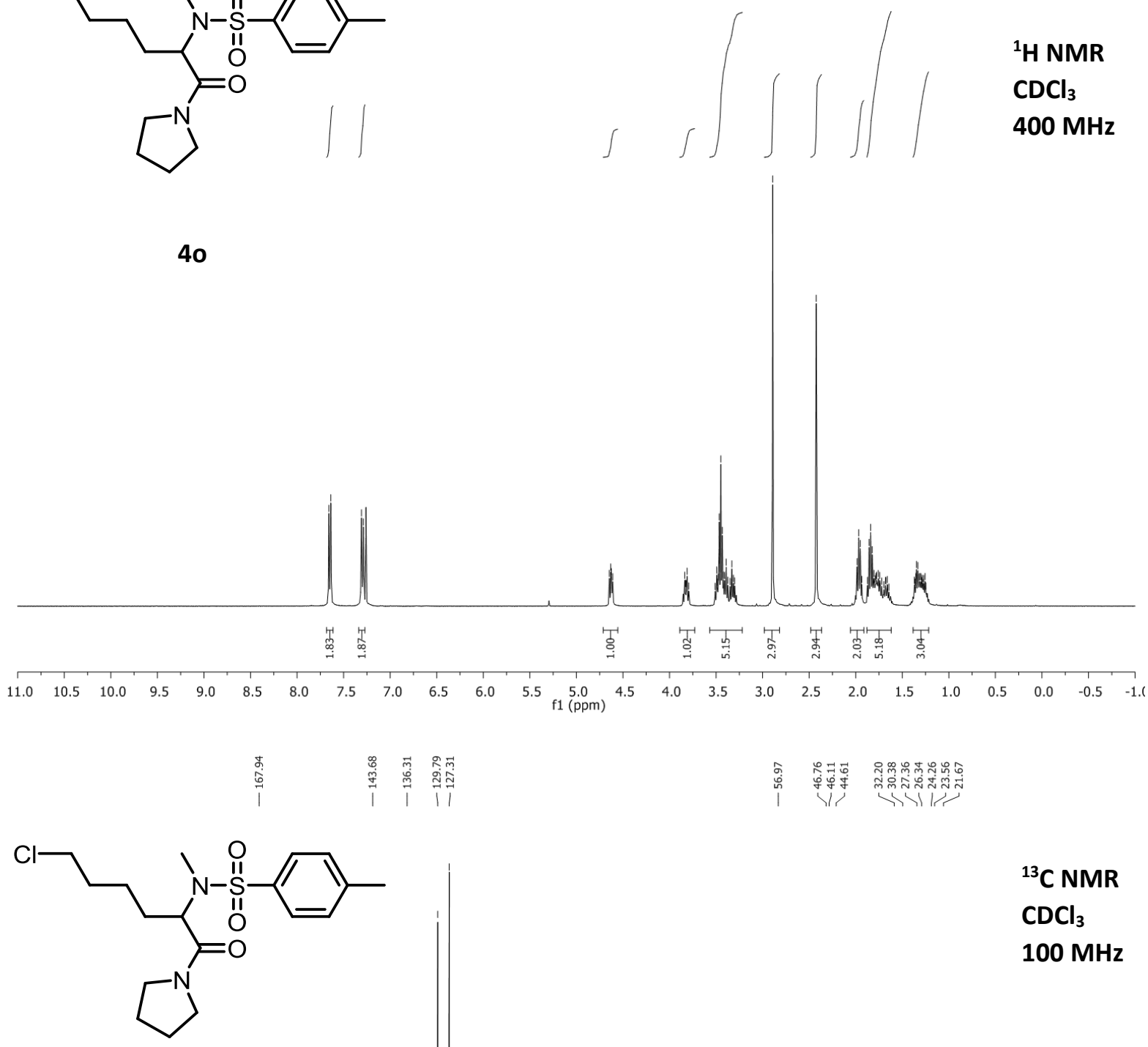

${ }^{13}$ C NMR

$\mathrm{CDCl}_{3}$

$100 \mathrm{MHz}$

40

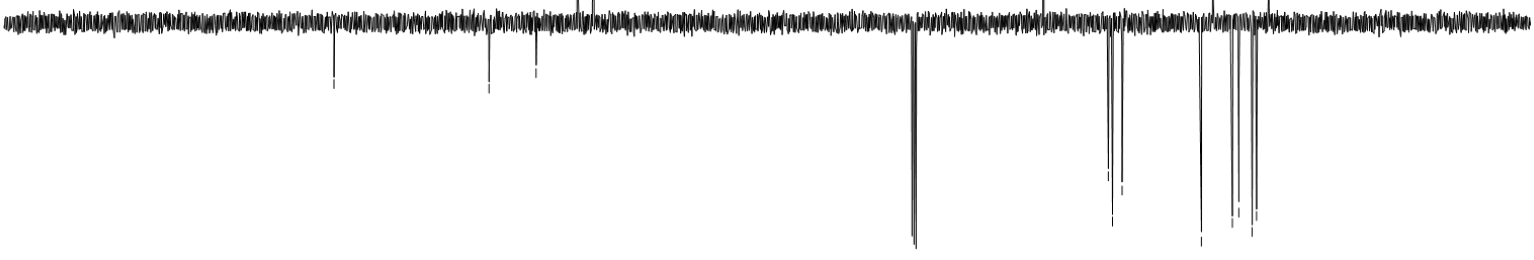

$\begin{array}{lllllllllllllllllllllll}210 & 200 & 190 & 180 & 170 & 160 & 150 & 140 & 130 & 120 & 110 & \begin{array}{c}100 \\ \mathrm{f} 1(\mathrm{ppm})\end{array} & 90 & 80 & 70 & 60 & 50 & 40 & 30 & 20 & 10 & 0 & -10\end{array}$ 

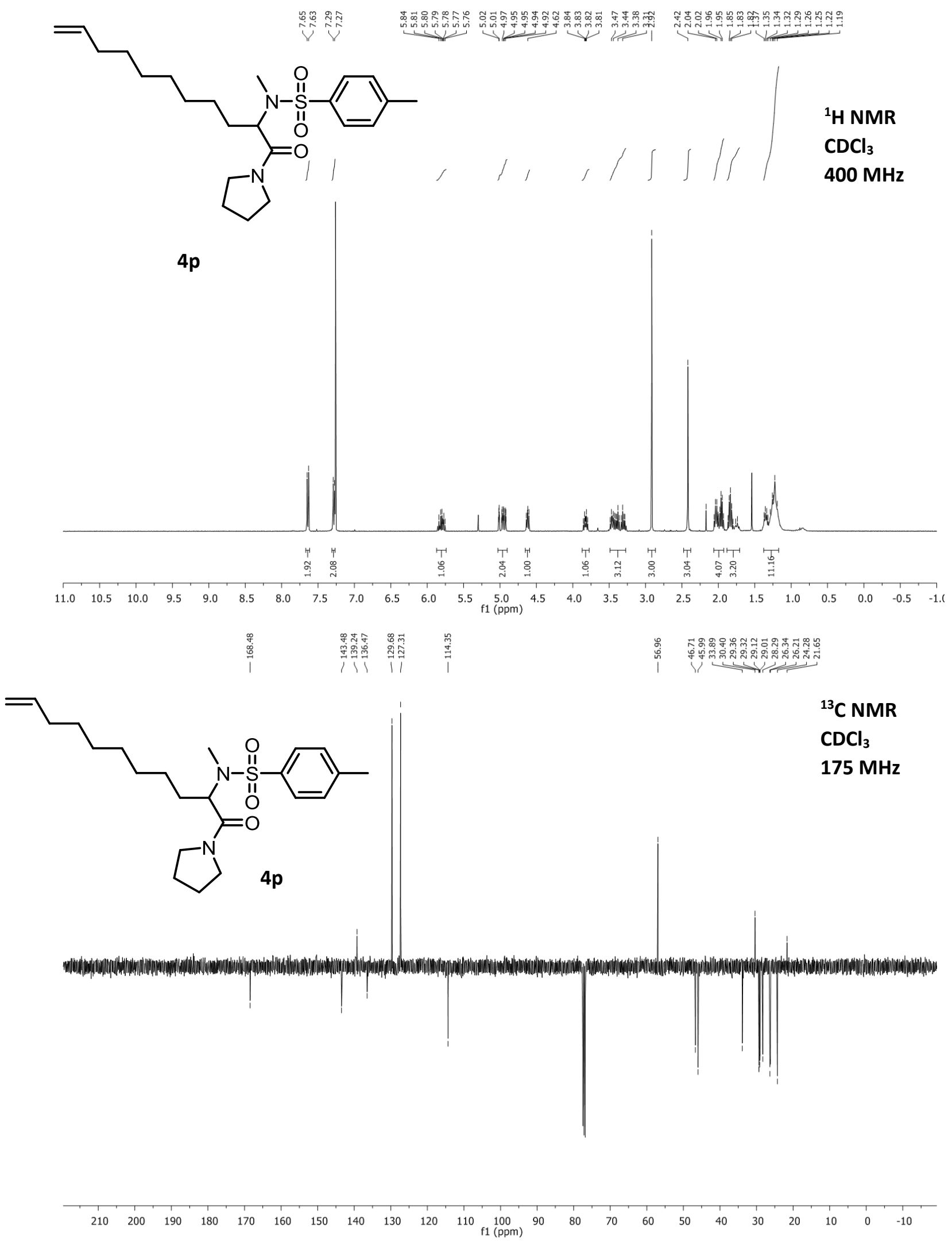


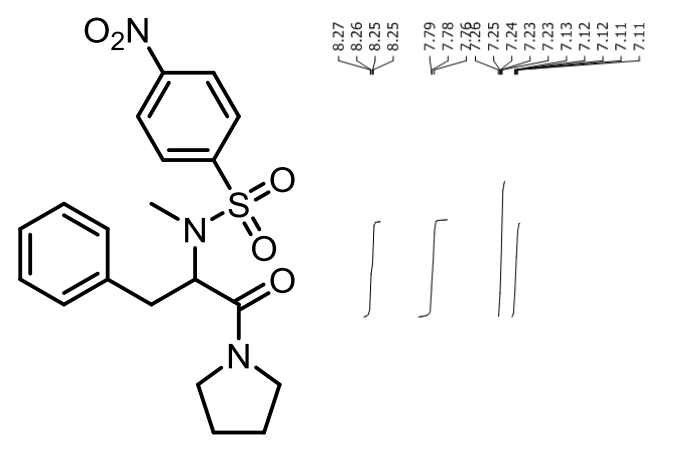

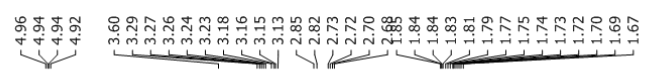

$4 q$
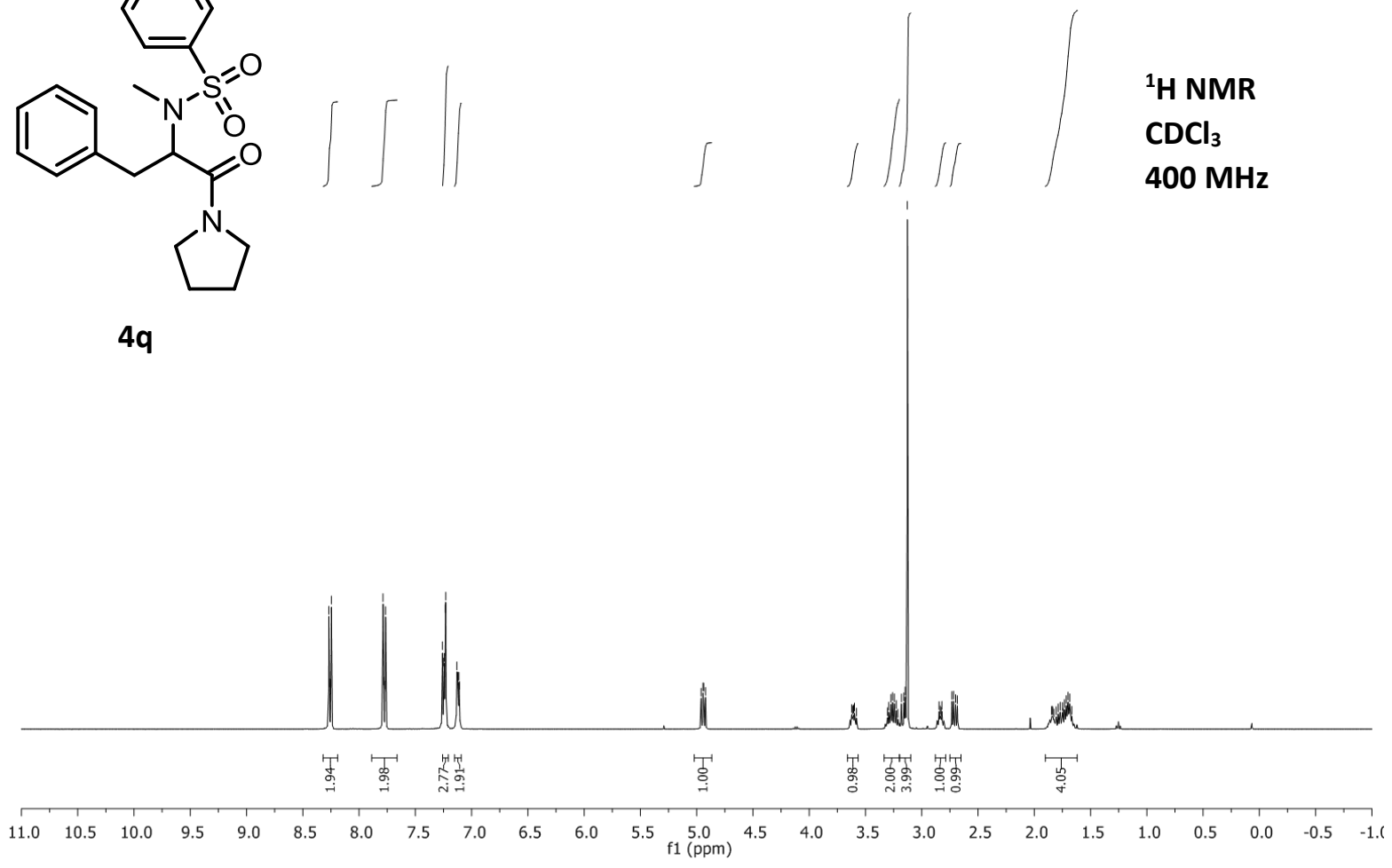<smiles>CN(C(Cc1ccccc1)C(=O)N1CCCC1)S(=O)(=O)c1ccc([N+](=O)[O-])cc1</smiles>

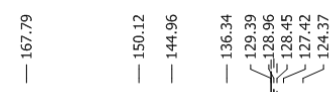

莣

$4 q$

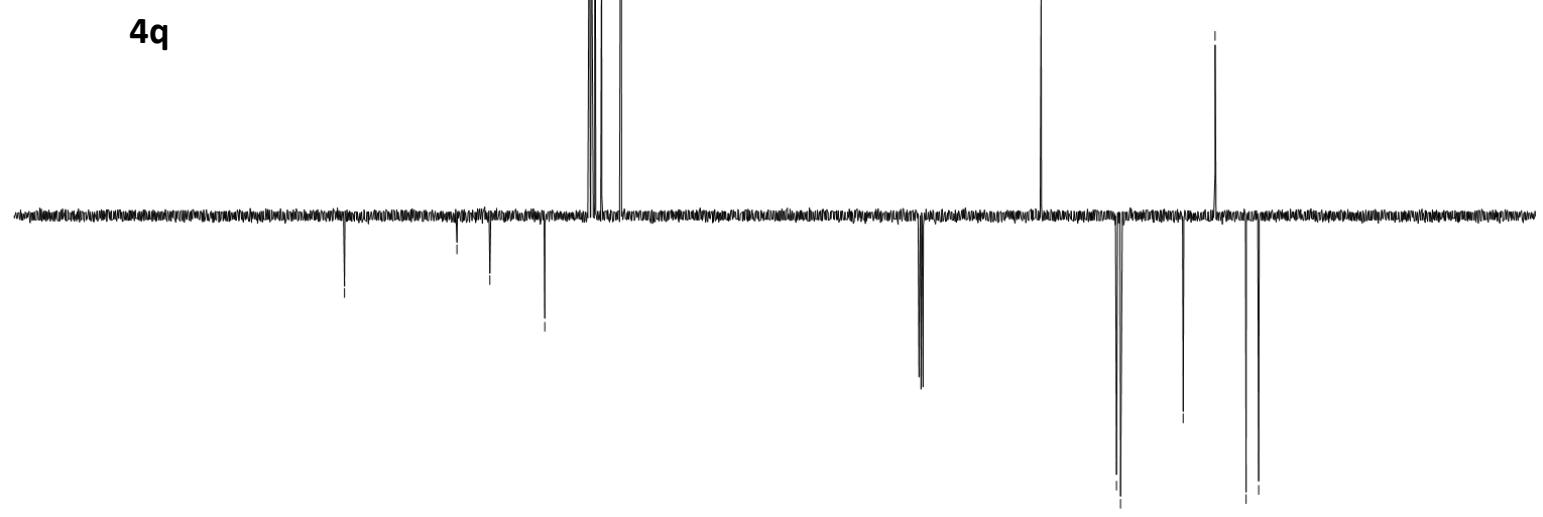

$\begin{array}{llllllllllllllllllllllllllllllll}210 & 200 & 190 & 180 & 170 & 160 & 150 & 140 & 130 & 120 & 110 & 100 & 90 & 80 & 70 & 60 & 50 & 40 & 30 & 20 & 10 & 0 & -10\end{array}$ 


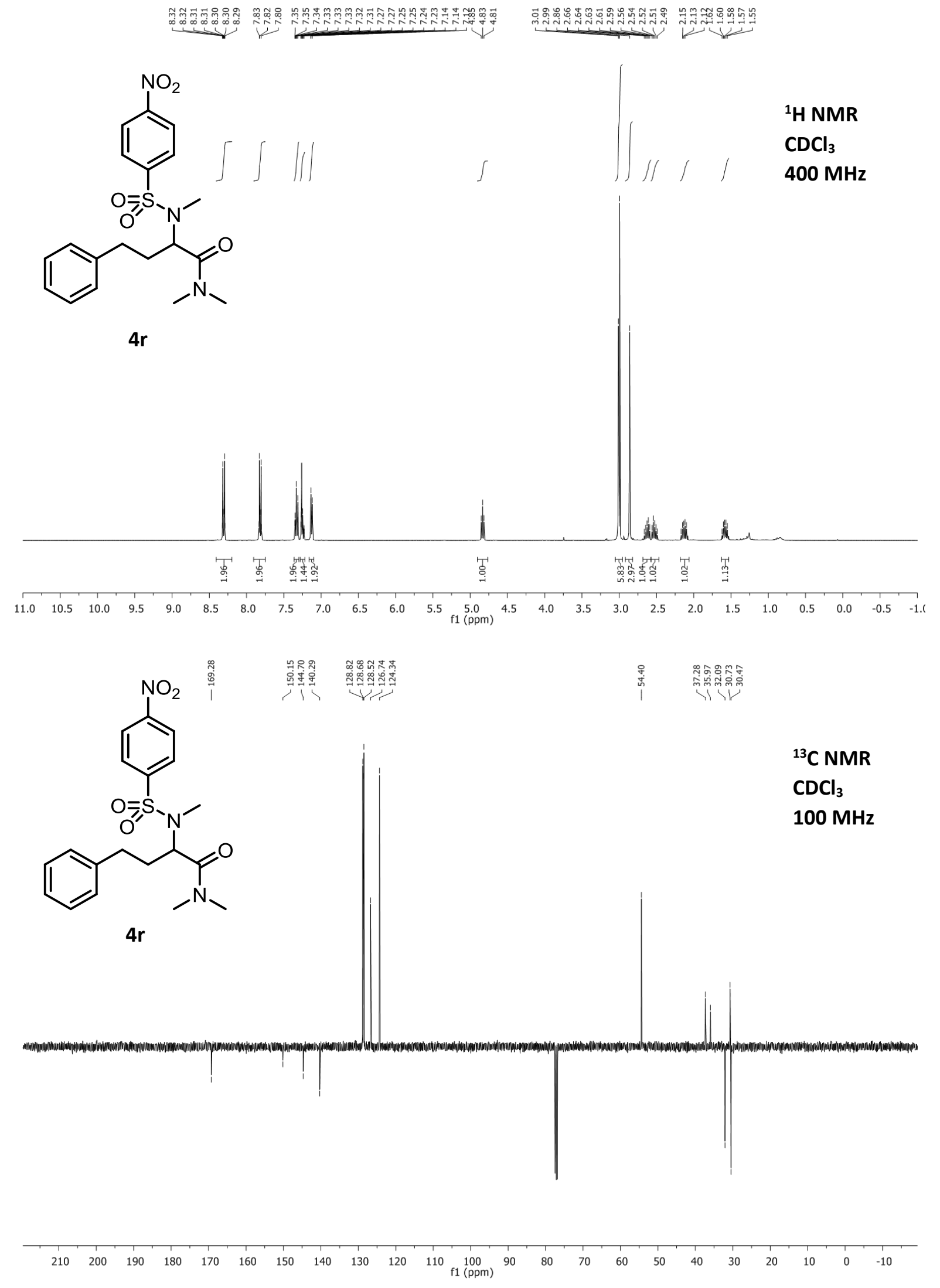


<smiles>CCCC(C(=O)N1CCOCC1)N(C)S(=O)(=O)c1ccc(C)cc1</smiles>

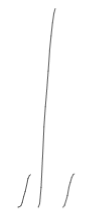

${ }^{1} \mathrm{H}$ NMR

$\mathrm{CDCl}_{3}$

4s

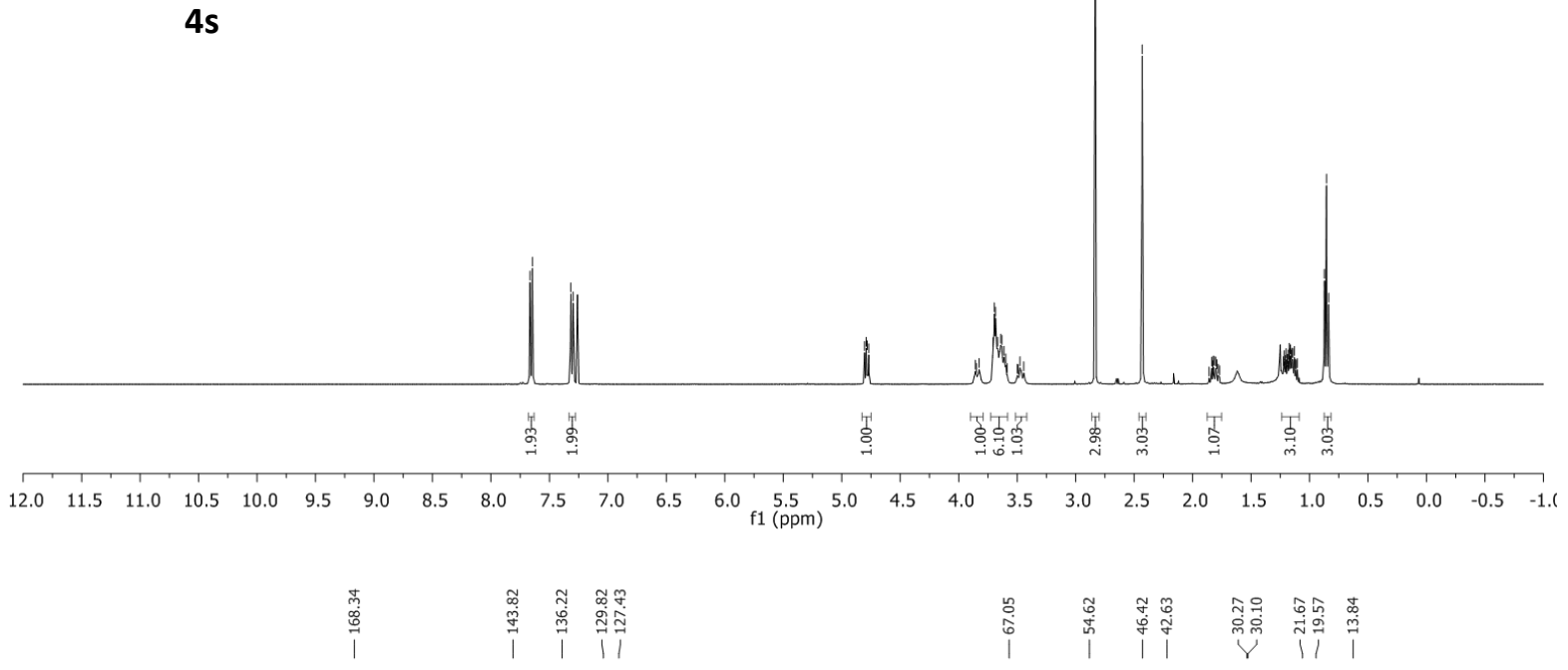<smiles>CCCC(C(=O)N1CCOCC1)N(C)S(=O)(=O)c1ccc(C)cc1</smiles>

${ }^{13}$ C NMR

$\mathrm{CDCl}_{3}$ $100 \mathrm{MHz}$

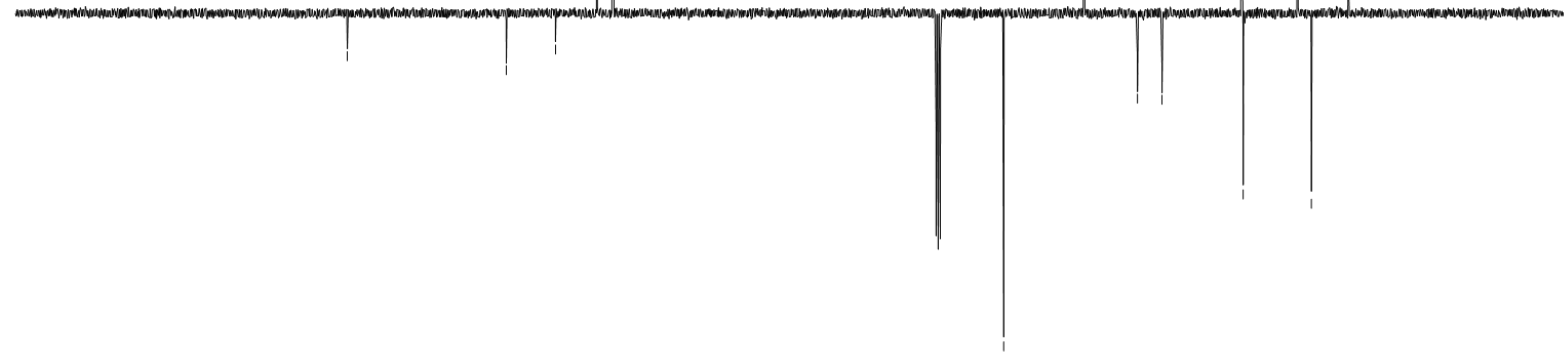

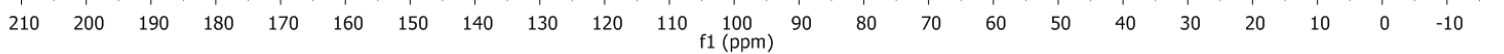




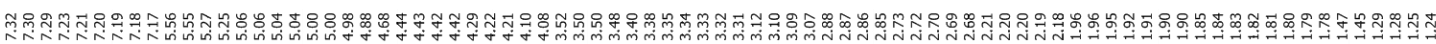
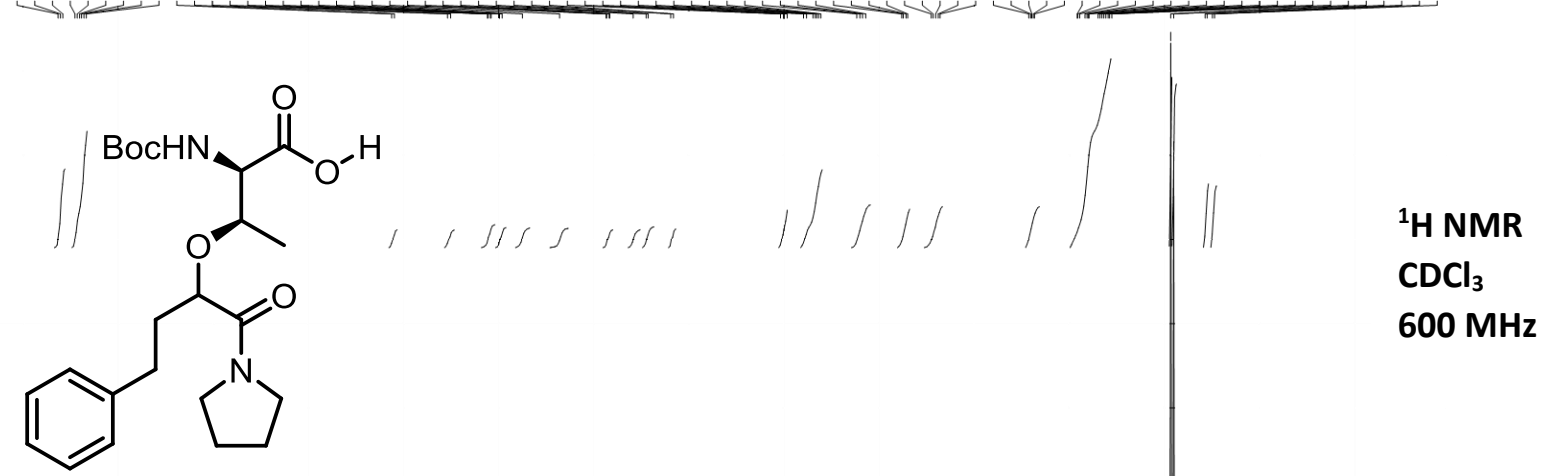

$5 a$
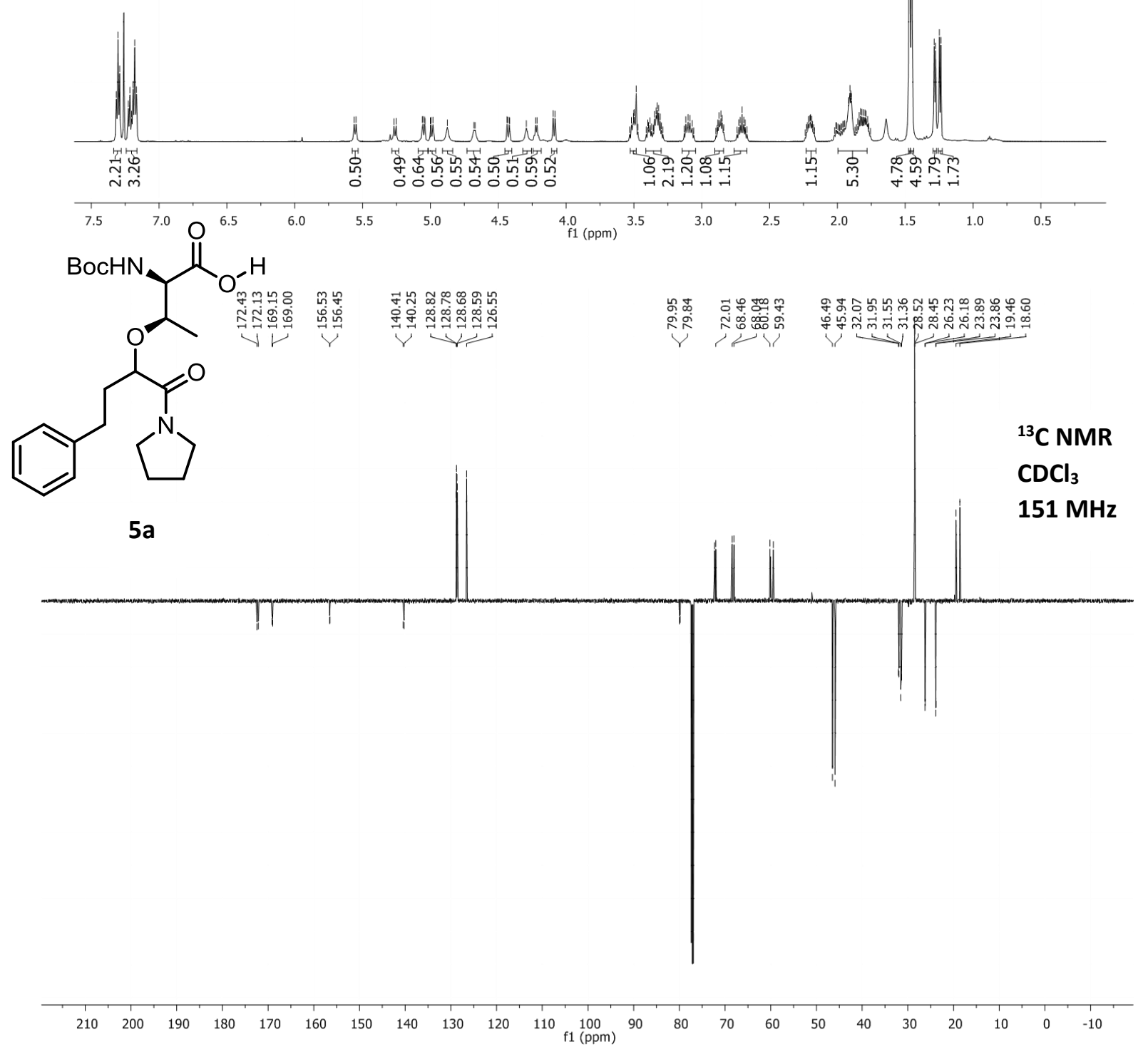


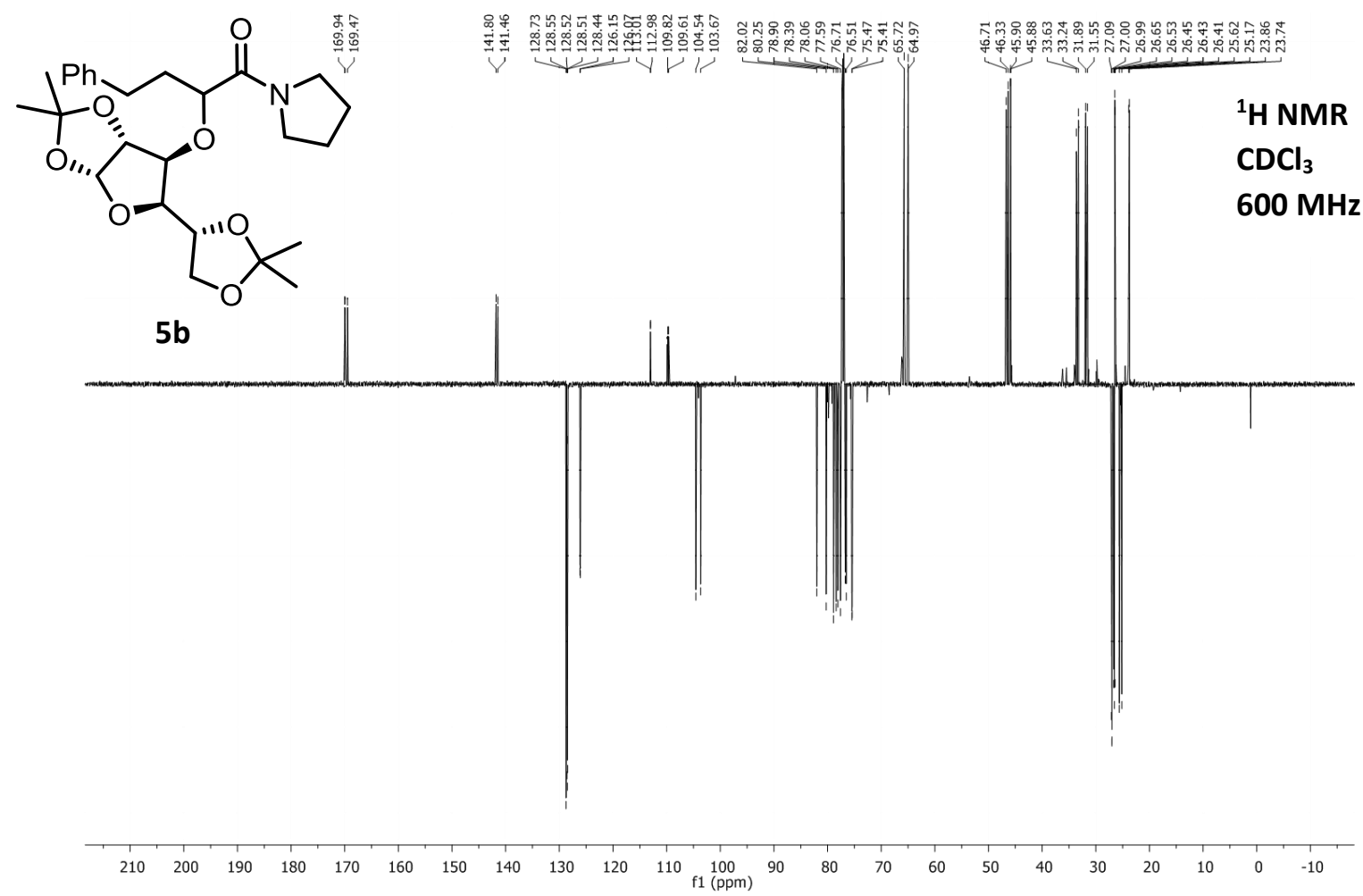

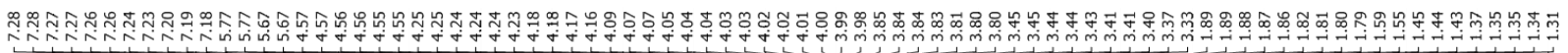

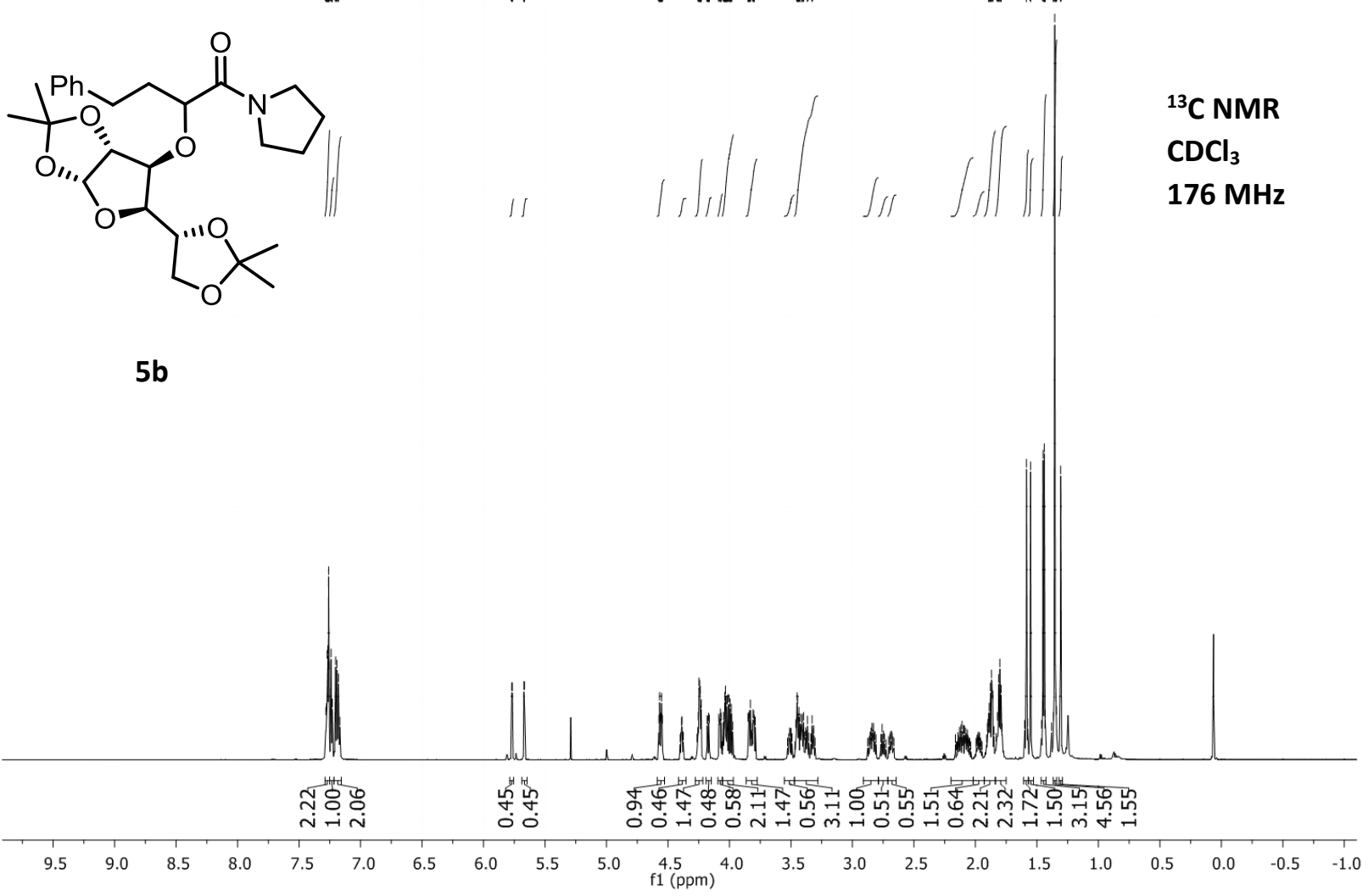




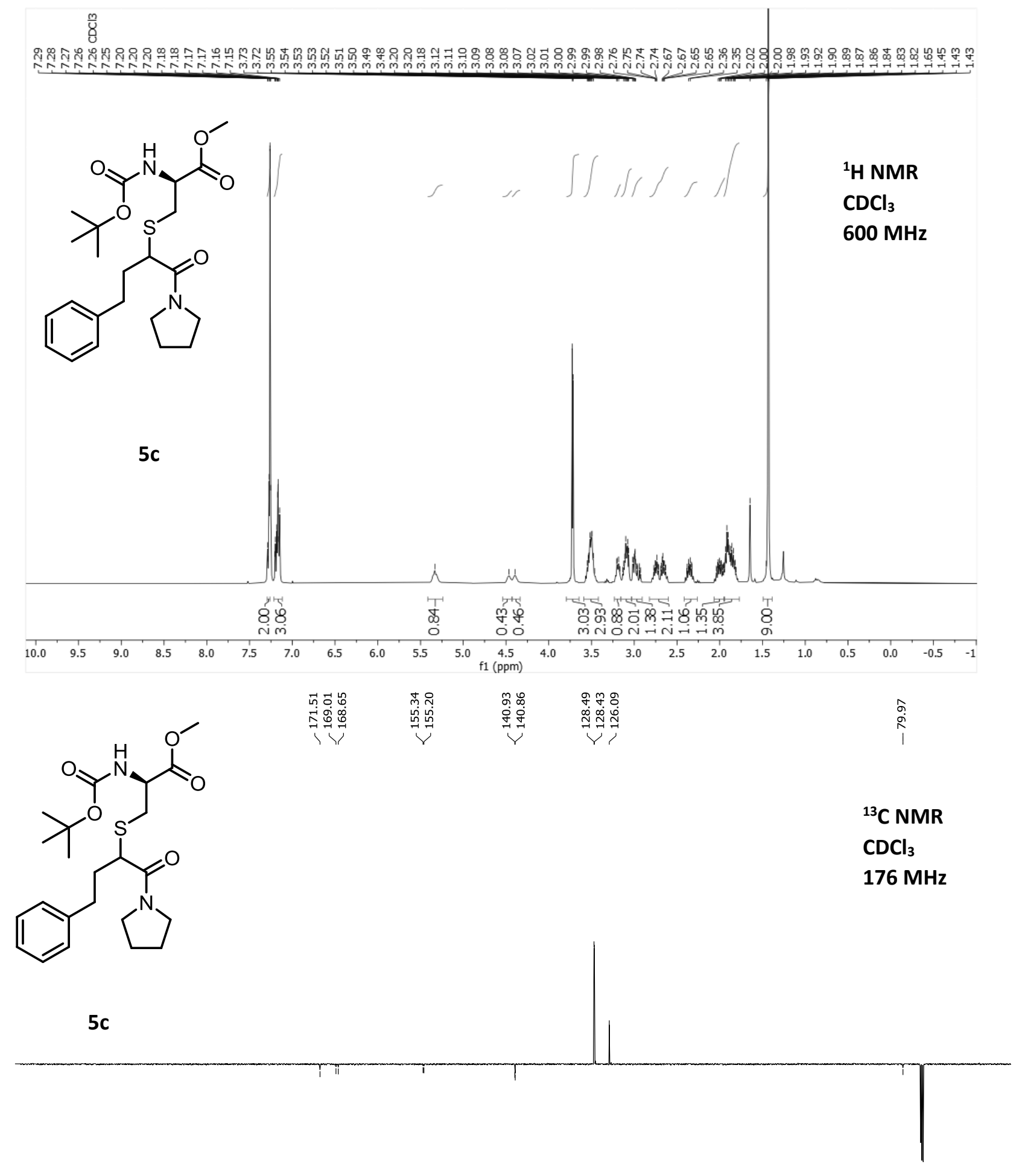

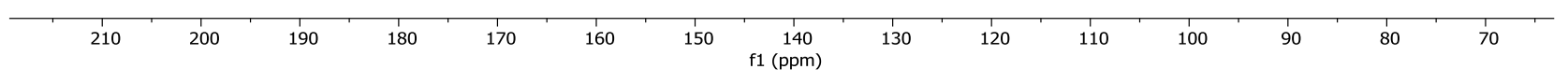



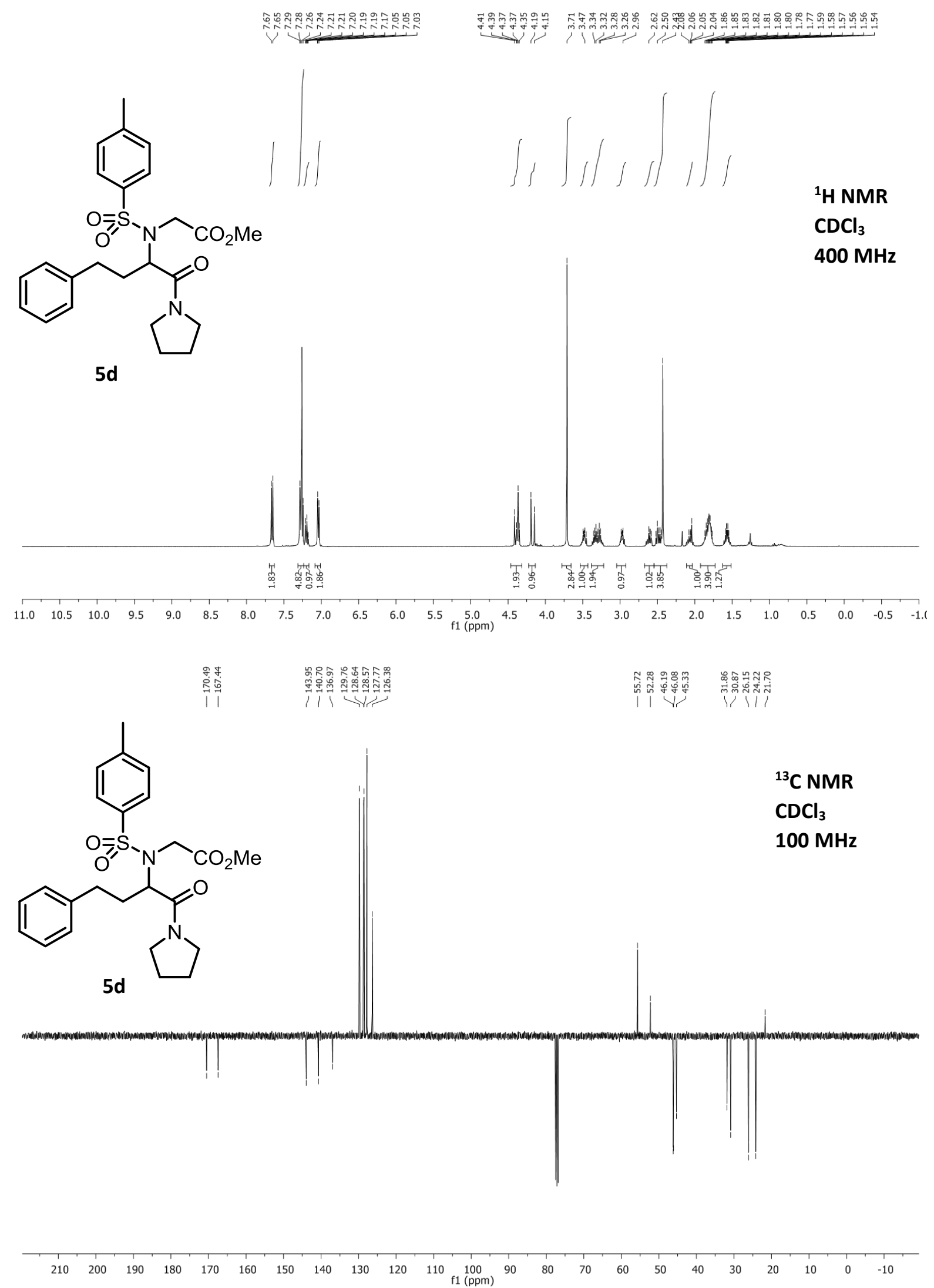
<smiles>NC(CCc1ccccc1)C(=O)N1CCCC1</smiles>

6
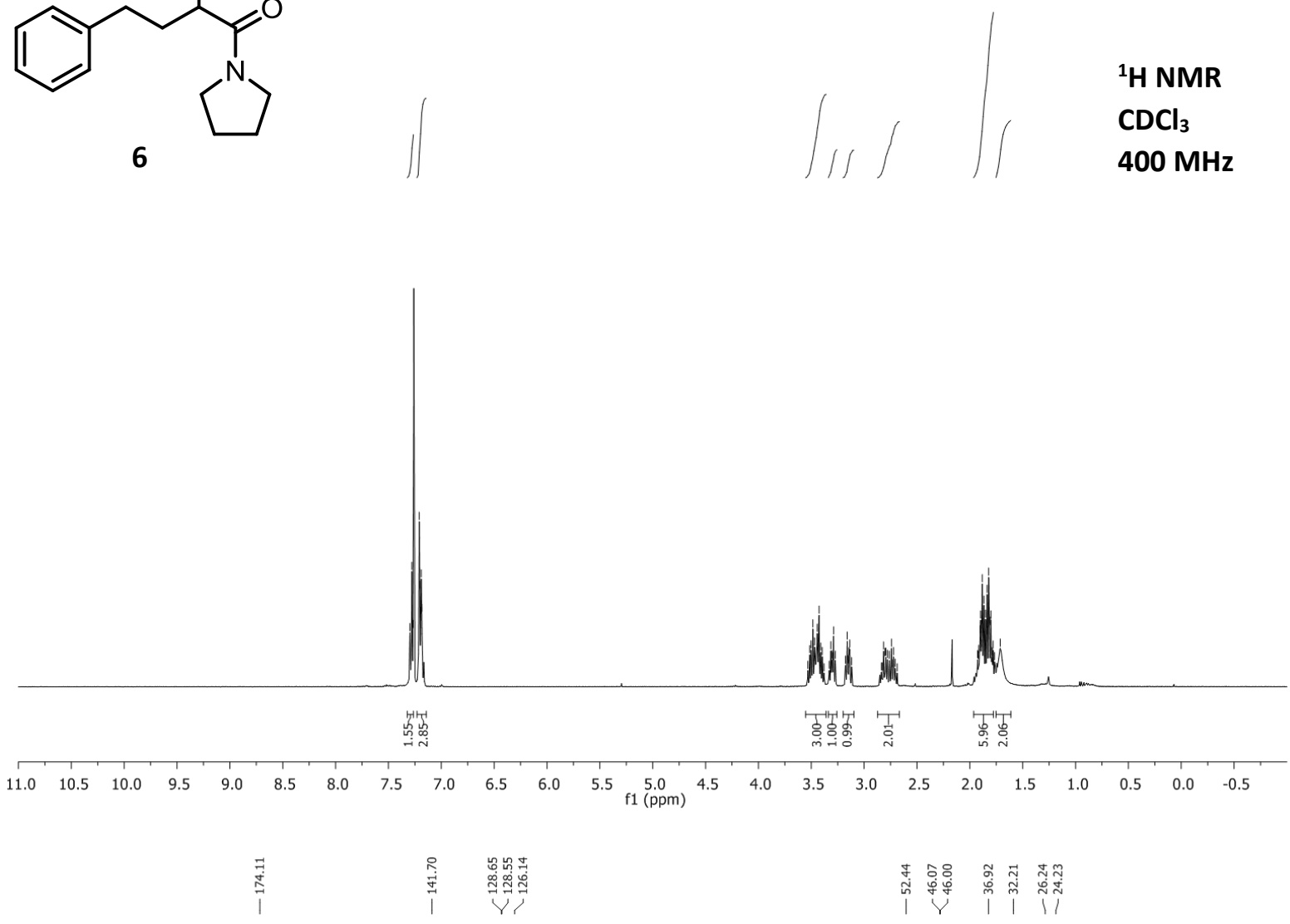<smiles>NC(CCc1ccccc1)C(=O)N1CCCC1</smiles>

${ }^{13} \mathrm{C}$ NMR

$\mathrm{CDCl}_{3}$ $100 \mathrm{MHz}$

6

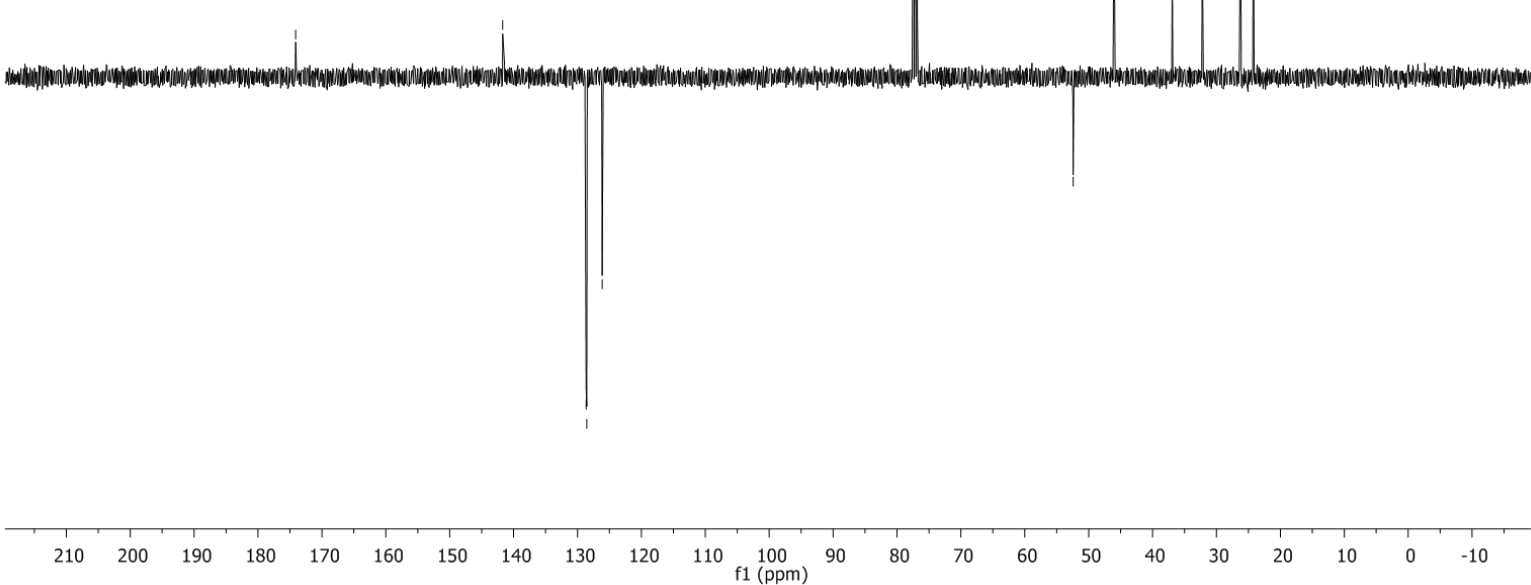



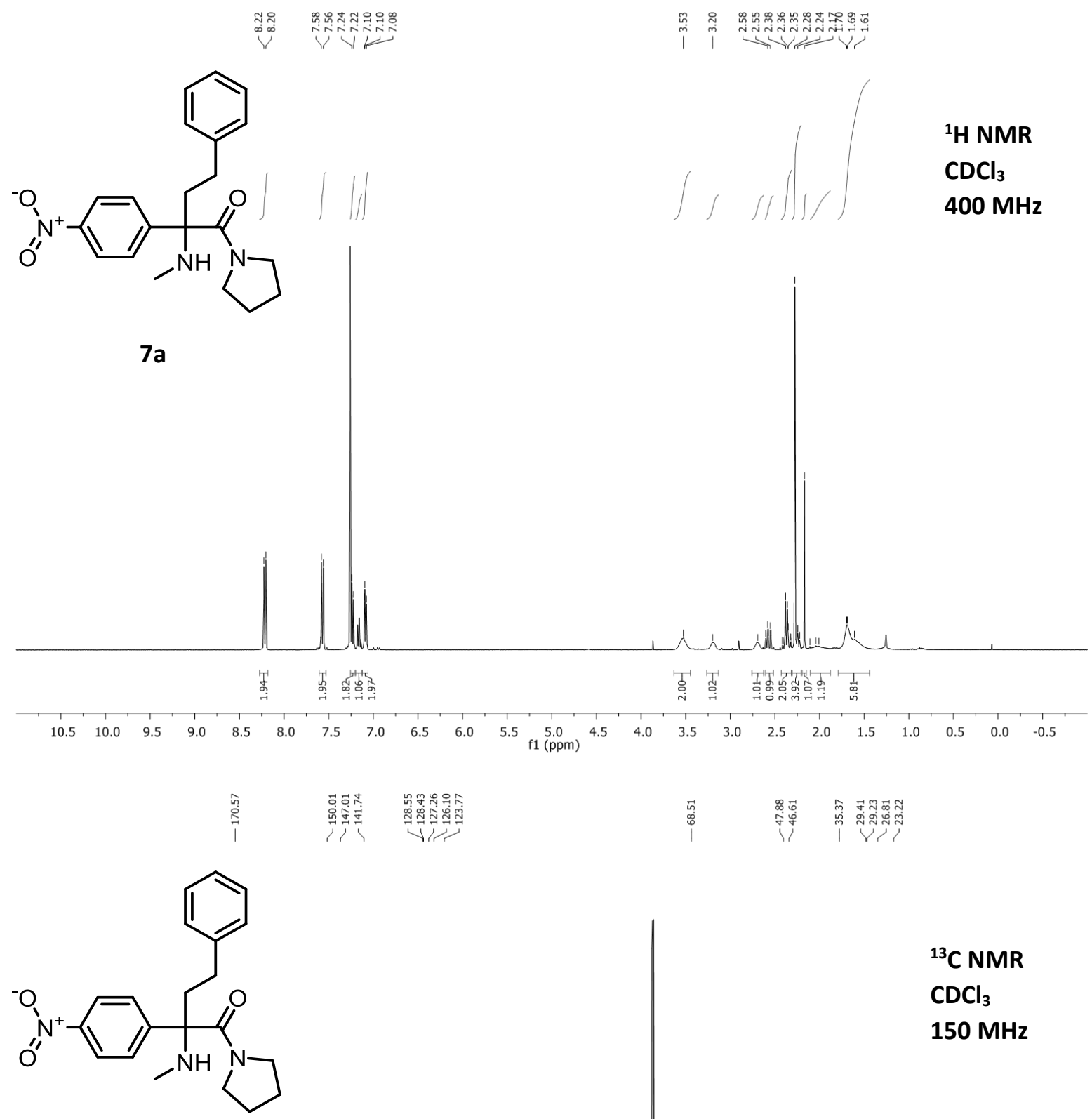

${ }^{13}$ C NMR

$\mathrm{CDCl}_{3}$ $150 \mathrm{MHz}$

$7 a$

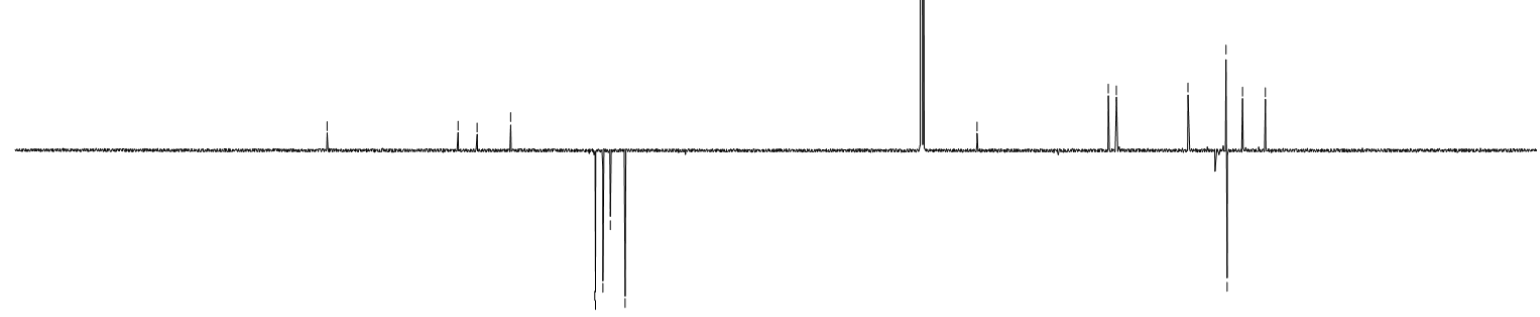

$\begin{array}{lllllllllllllllllllllllllllllllllll}210 & 200 & 190 & 180 & 170 & 160 & 150 & 140 & 130 & 120 & 110 & 100 & 90 & 80 & 70 & 60 & 50 & 40 & 30 & 20 & 10 & 0 & -10\end{array}$ 


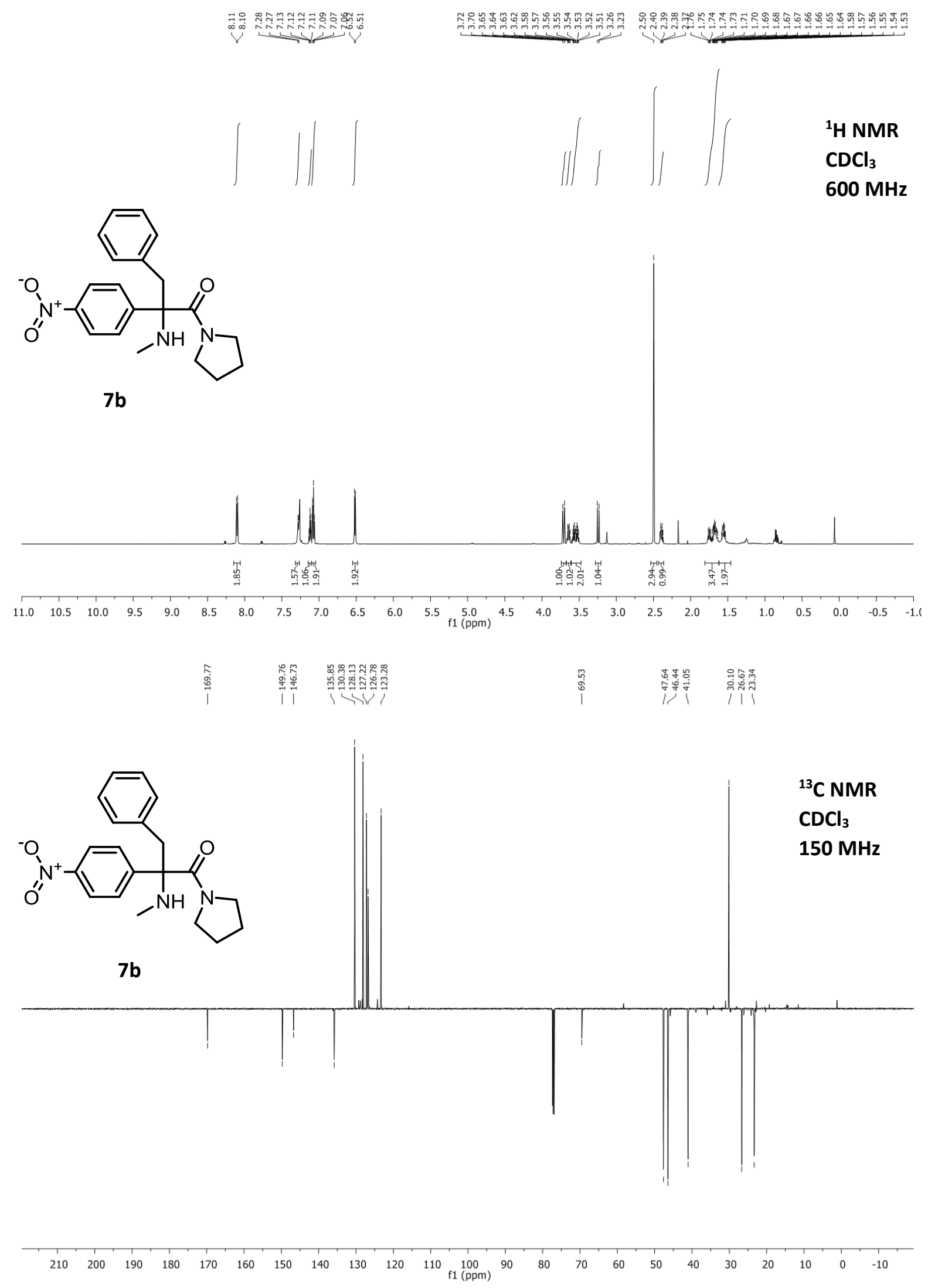



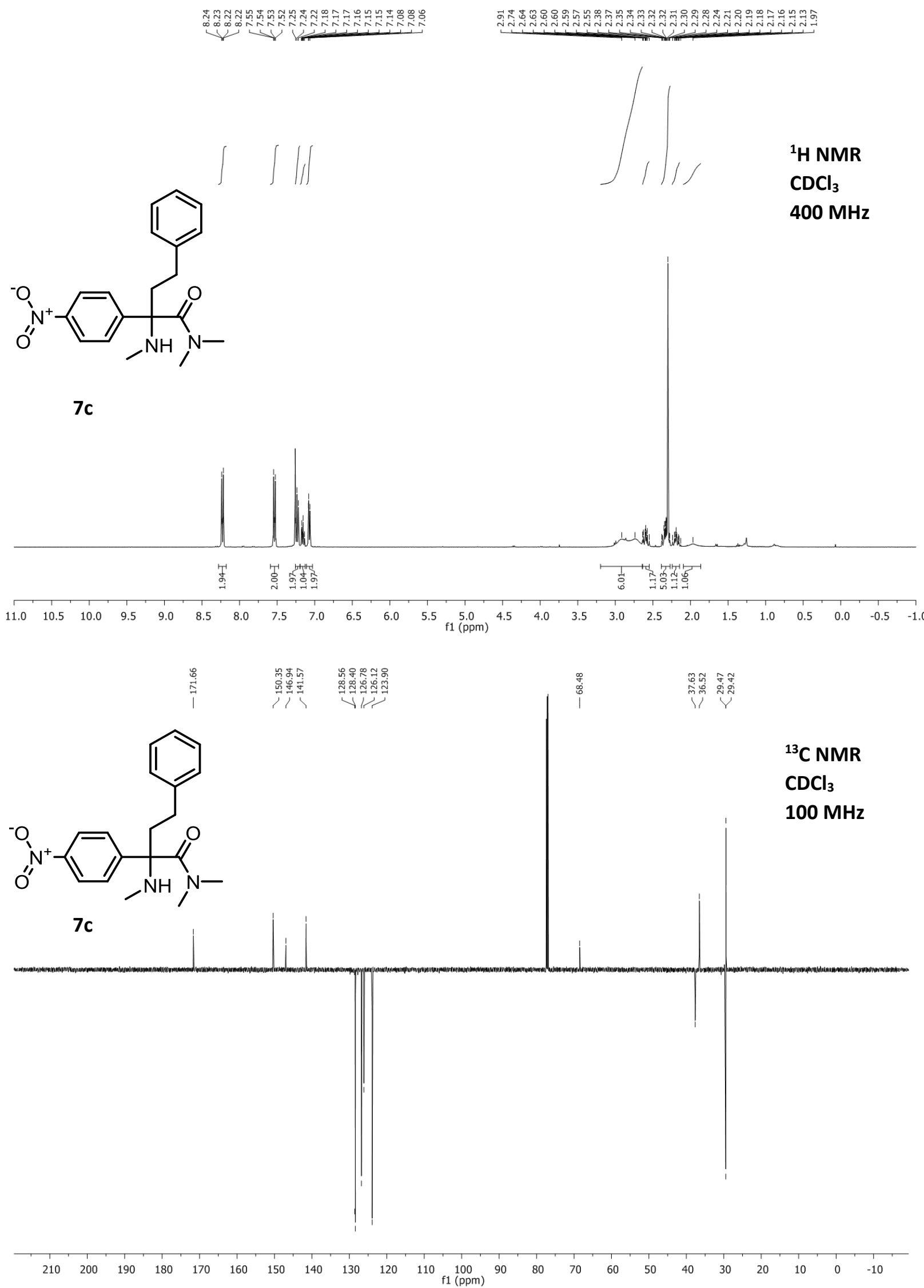


\section{References}

[1] Peng, B.; Geerdink, D.; Farès, C.; Maulide, N. Chemoselective Intermolecular $\alpha$-Arylation of Amides. Angew. Chem. Int. Ed. 2014, 53, 5462-5466.

[2] Zhou, X.; Zhang, G.; Gao, B.; Huang, H. Palladium-Catalyzed Hydrocarbonylative C-N Coupling of Alkenes with Amides. Org. Lett., 2018, 20, 2208-2212.

[3] Tona, V.; de la Torre, A.; Padmanaban, M.; Ruider, S.; González, L.; Maulide, N. Chemo- and Stereoselective Transition-Metal-Free Amination of Amides with Azides. J. Am. Chem. Soc. 2014, 138, 8348-8351.

[4] Zhang, F. et al. Cu-Catalyzed Cascades to Carbocycles: Union of Diaryliodonium Salts with Alkenes or Alkynes Exploiting Remote Carbocations. J. Am. Chem. Soc. 2014, 136, 8851-8854.

[5] de la Torre, A.; Kaiser, D.; Maulide, N. Flexible and Chemoselective Oxidation of Amides to $\alpha$-Keto Amides and $\alpha$-Hydroxy Amides. J. Am. Chem. Soc. 2017, 139, 6578-6581.

[6] Zultanski, S. L.; Zhao, J.; Stahl S. S. Practical Synthesis of Amides via Copper/ABNO-Catalyzed Aerobic Oxidative Coupling of Alcohols and Amines. J. Am. Chem. Soc. 2016, 138, 6416-6419.

[7] Feldman, K. S.; Folda, T. S. Studies on the Synthesis of the Alkaloid (-)-Gilbertine via Indolidene Chemistry. J. Org. Chem. 2016, 81, 4566-4575.

[8] Saidykhan, A.; Bowen, R. D.; Gallagher, R. T.; Martin, W. H. C. Intramolecular NC Rearrangements Involving Sulfonamide Protecting Groups. Tetrahedron Lett. 2015, 56, 66-68.

[9] Gioiello, A.; Rosatelli, E.; Teofrasti, M.; Filipponi, P.; Pellicciari, R. Building a Sulfonamide Library by EcoFriendly Flow Synthesis. ACS Comb. Sci. 2013, 15, 235-239.

[10] Chow, S. Y.; Stevens, M. Y.; Odell, L. R. Sulfonyl Azides as Precursors in Ligand-Free Palladium-Catalyzed Synthesis of Sulfonyl Carbamates and Sulfonyl Ureas and Synthesis of Sulfonamides. J. Org. Chem. 2016, $81,2681-2691$.

[11] Brockway, A. J.; Cosner, C. C.; Volkov, O. A.; Phillips, M. A.; De Brabander, J. K. Improved Synthesis of MDL 73811 - A Potent AdoMetDC Inhibitor and Anti-Trypanosomal Compound. Synthesis 2016, 48: 20652068.

[12] Liang, R.; Li, S.; Wang, R.; Lu, L.; Li, F. N-Methylation of Amines with Methanol Catalyzed by a Cp*Ir Complex Bearing a Functional 2,2'-Bibenzimidazole Ligand. Org. Lett. 2017, 19, 5790-5793.

[13] Wright, S. W.; Hallstrom, K. N. A Convenient Preparation of Heteroaryl Sulfonamides and Sulfonyl Fluorides from Heteroaryl Thiols. J. Org. Chem. 2006, 71, 1080-1084. 


\section{X-ray Analysis}

The X-ray intensity data were measured on Bruker X8 APEX2 diffractometer equipped with multilayer monochromator, Mo K/a INCOATEC micro focus sealed tube and Cryoflex cooling device. The structure was solved by direct methods and refined by full-matrix least-squares techniques. Non-hydrogen atoms were refined with anisotropic displacement parameters. Hydrogen atoms were inserted at calculated positions and refined with riding model. The following software was used: Bruker SAINT software package $e^{b}$ using a narrow-frame algorithm for frame integration, $S A D A B S^{\mathrm{c}}$ for absorption correction, $O L E X 2^{d}$ for structure solution, refinement, molecular diagrams and graphical user-interface, Shelxle for refinement and graphical user-interface SHELXS-2015 for structure solution, SHELXL-2015 $5^{\mathrm{g}}$ for refinement, Platon ${ }^{h}$ for symmetry check. Experimental data and CCDCCodes Experimental data and CCDC-Code (Available online: http://www.ccdc.cam.ac.uk/conts/retrieving.html) can be found in Table S1. Crystal data, data collection parameters, and structure refinement details are given in Tables S2 and S3. Crystal structure is visualized in Figure S1.

Table S1 Experimental parameter and CCDC-Code.

\begin{tabular}{|c|c|c|c|c|c|c|c|c|}
\hline Sample & Machine & Source & Temp. & $\begin{array}{c}\text { Detector } \\
\text { Distance }\end{array}$ & $\begin{array}{c}\text { Time/ } \\
\text { Frame }\end{array}$ & \#Frames & $\begin{array}{c}\text { Frame } \\
\text { width }\end{array}$ & CCDC \\
\hline & Bruker & & {$[\mathrm{K}]$} & {$[\mathrm{mm}]$} & {$[\mathrm{s}]$} & & {$\left[{ }^{\circ}\right]$} & \\
\hline $7 \mathbf{c}$ & $\mathrm{X} 8$ & Mo & 100 & 35 & 4 & 1073 & 0.500 & 1898975 \\
\hline
\end{tabular}

\footnotetext{
${ }^{\mathrm{b}}$ Bruker SAINT v8.38B Copyright (C) 2005-2019 Bruker AXS

${ }^{c}$ Sheldrick, G. M. (1996). SADABS. University of Göttingen, Germany.

d Dolomanov, O.V., Bourhis, L.J., Gildea, R.J, Howard, J.A.K. \& Puschmann, H. , OLEX2, (2009), J. Appl. Cryst. 42, 339-341

${ }^{\text {e }}$ C. B. Huebschle, G. M. Sheldrick and B. Dittrich, ShelXle: a Qt graphical user interface for SHELXL, J. Appl. Cryst., 44, (2011) 12811284

f Sheldrick, G. M. (2015). SHELXS v 2016/4 University of Göttingen, Germany.

g Sheldrick, G. M. (2015). SHELXL v 2016/4 University of Göttingen, Germany.

h A. L. Spek, Acta Cryst. 2009, D65, 148-155.
} 


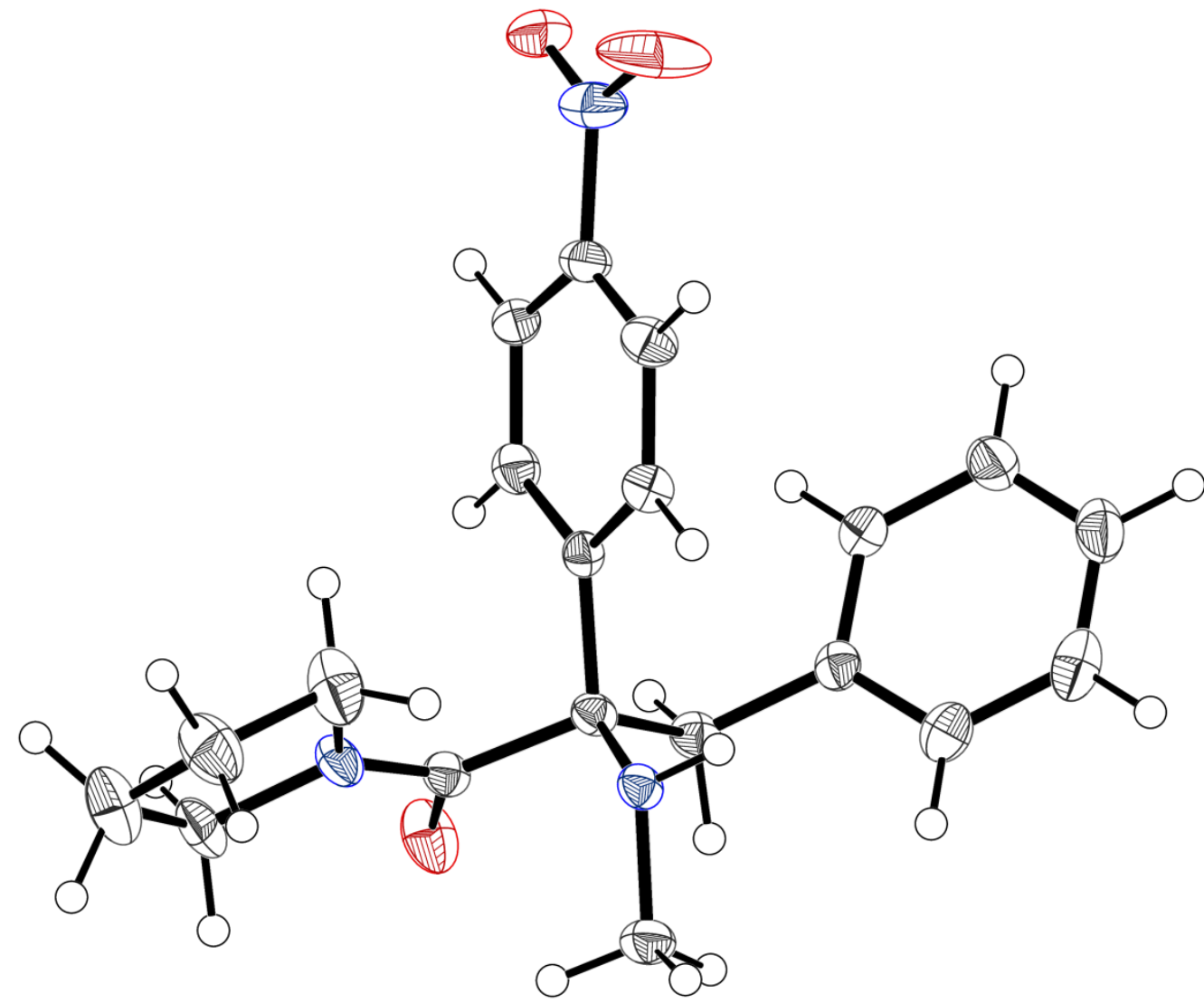

Figure S1 Crystal structure, drawn with $50 \%$ displacement ellipsoid. The bond precision for C-C single bonds is $0.0048 \AA$. The chiral orientation could not be determined (visualized R).

Table S2 Sample and crystal data.

\begin{tabular}{|c|c|c|c|c|}
\hline Chemical formula & $\mathrm{C} 20 \mathrm{H} 23 \mathrm{~N} 3 \mathrm{O} 3$ & Crystal system & \multicolumn{2}{|c|}{ orthorhombic } \\
\hline Formula weight $[\mathrm{g} / \mathrm{mol}]$ & 353.41 & Space group & \multicolumn{2}{|c|}{$P 212121$} \\
\hline Temperature $[\mathrm{K}]$ & 100 & $\mathbf{Z}$ & \multicolumn{2}{|c|}{4} \\
\hline Measurement method & $\backslash f$ and $\backslash w$ scans & Volume $\left[\AA^{3}\right]$ & \multicolumn{2}{|c|}{$1819.4(2)$} \\
\hline $\begin{array}{l}\text { Radiation (Wavelength } \\
[\AA \AA]]\end{array}$ & $\operatorname{MoK} \alpha(\lambda=0.71073)$ & $\begin{array}{l}\text { Unit cell } \\
\text { dimensions [̊̊] } \\
\text { and }\left[^{\circ}\right]\end{array}$ & $6.3111(4)$ & 90 \\
\hline Crystal size / $\left[\mathbf{m m}^{3}\right]$ & $0.35 \times 0.18 \times 0.07$ & & $9.3707(5)$ & 90 \\
\hline Crystal habit & clear colourless plate & & $30.764(3)$ & 90 \\
\hline $\begin{array}{l}\text { Density (calculated) / } \\
{\left[\mathrm{g} / \mathrm{cm}^{3}\right]}\end{array}$ & 1.29 & $\begin{array}{l}\text { Absorption } \\
\text { coefficient } /\left[\mathrm{mm}^{-1}\right]\end{array}$ & \multicolumn{2}{|c|}{0.088} \\
\hline Abs. correction Tmin & 0.97 & $\begin{array}{l}\text { Abs. correction } \\
\text { Tmax }\end{array}$ & \multicolumn{2}{|c|}{0.994} \\
\hline Abs. correction type & multiscan & $F(000)\left[e^{-}\right]$ & \multicolumn{2}{|c|}{752} \\
\hline
\end{tabular}


Table S3 Data collection and structure refinement.

\begin{tabular}{|c|c|c|c|c|}
\hline Index ranges & $\begin{array}{c}-7 \leq \mathrm{h} \leq 7,-11 \leq \mathrm{k} \leq 11 \\
-36 \leq 1 \leq 37\end{array}$ & $\begin{array}{l}\text { Theta range for } \\
\text { data collection }\left[{ }^{\circ}\right]\end{array}$ & & .544 to 50.68 \\
\hline Reflections number & 13667 & $\begin{array}{l}\text { Data / restraints / } \\
\text { parameters }\end{array}$ & & $3331 / 0 / 239$ \\
\hline Refinement method & Least squares & \multirow{2}{*}{ Final $R$ indices } & all data & $\mathrm{R} 1=0.0572, \mathrm{wR} 2=0.1099$ \\
\hline Function minimized & $\Sigma \mathrm{w}\left(\mathrm{F}_{\mathrm{o}}^{2}-\mathrm{F}_{\mathrm{c}}^{2}\right)^{2}$ & & $\mathrm{I}>2 \sigma(\mathrm{I})$ & $\mathrm{R} 1=0.0460, \mathrm{wR} 2=0.1023$ \\
\hline Goodness-of-fit on $F^{2}$ & 1.063 & \multirow[b]{2}{*}{ Weighting scheme } & \multicolumn{2}{|c|}{$\mathrm{w}=1 /\left[\sigma^{2}\left(\mathrm{~F}_{\mathrm{o}}^{2}\right)+(0.0264 \mathrm{P})^{2}+0.7427 \mathrm{P}\right]$} \\
\hline $\begin{array}{l}\text { Largest diff. peak and } \\
\text { hole }\left[e \AA^{-3}\right]\end{array}$ & $0.29 /-0.21$ & & \multicolumn{2}{|c|}{ where $\mathrm{P}=\left(\mathrm{Fo}_{\mathrm{o}}^{2}+2 \mathrm{~F}_{\mathrm{c}}^{2}\right) / 3$} \\
\hline
\end{tabular}




\section{Computational details}

The conformational space of all flexible molecules has been initially searched using the OPLS_2005 force field ${ }^{1}$ and the systematic Monte Carlo conformers search routine implemented in MACROMODEL 11.5. ${ }^{2}$

To consider the flexibility of the complexes composed of individual fragments (e.g. the complex of a cation with the negatively charged $\mathrm{TfO}^{-}$counterion), the electrostatic potential of the ions has been studied applying natural bond orbital (NBO) population analysis. The reciprocal positions of the fragments have been determined based on the calculated NBO charges. The obtained complexes have been used to restrict further the conformational search and obtain the set of complexes that will be reoptimized using density functional theory (DFT) methods.

Accordingly, the structures located at force field level have then been subjected to B3LYP-D3/def2-SVP ${ }^{3-8}$ (using def2-ECP9 for iodine atom) geometry optimization. The "Ultrafine" integration grid has been applied. The nature of all stationary points (minima and transition states) was verified through the computation of the vibrational frequencies. The thermal corrections to the Gibbs free energy were combined with the single point energies calculated at the DLPNO-CCSD(T)/def2-TZVP ${ }^{10,11}$ (using "TightPNO" settings) to yield DLPNO-CCSD(T)//DFT Gibbs free energies (" $\mathrm{G}_{298}$ ") at $298.15 \mathrm{~K}$. All energies are reported in $\mathrm{kcal} \mathrm{mol}^{-1}$.

The density-based solvation model SMD ${ }^{12}$ was applied to consider solvent (DCM) effects for both geometries and energies. Solvation factors (for the Gibbs free energies at the DLPNO-CCSD(T)//DFT level of theory) have been calculated by single point energies in gas phase of the optimized geometries in solution. Free energies in solution have been corrected to a reference state of $1 \mathrm{~mol} \mathrm{I}^{-1}$ at $298.15 \mathrm{~K}$ through addition of $\mathrm{RT} \ln (24.46)=+7.925 \mathrm{~kJ} \mathrm{~mol}^{-}$ ${ }^{1}$ to the gas phase ( $\left.1 \mathrm{~atm}\right)$ free energies.

The DFT calculations have been performed with the Gaussian09 program package. ${ }^{13}$ The ORCA 4.0.1 software was applied for the DLPNO-CCSD(T) computations. ${ }^{14}$

\section{References}

(1) Banks, J. L.; Beard, H. S.; Cao, Y.; Cho, A. E.; Damm, W.; Farid, R.; Felts, A. K.; Halgren, T. A.; Mainz, D. T.; Maple, J. R.; Murphy, R.; Philipp, D. M.; Repasky, M. P.; Zhang, L. Y.; Berne, B. J.; Friesner, R. A.; Gallicchio, E.; Levy, R. M. J. Comput. Chem. 2005, 26 (16), 1752-1780.

(2) MacroModel, Schrödinger, LLC, New York, NY, 2018.

(3) Becke, A. J. Chem. Phys. 1993, 98, 5648-5652.

(4) Lee, C.; Yang, W.; Parr, R. G. Phys. Rev. B 1988, 37 (2), 785-789.

(5) Vosko, S. H.; Wilk, L.; Nusair, M. Can. J. Phys. 1980, 58 (8), 1200-1211.

(6) Stephens, P. J.; Devlin, F. J.; Chabalowski, C. F.; Frisch, M. J. J. Phys. Chem. 1994, 98 (45), 11623-11627.

(7) Grimme, S.; Antony, J.; Ehrlich, S.; Krieg, H. J. Chem. Phys. 2010, 132 (15), 154104.

(8) Weigend, F.; Ahlrichs, R. Phys. Chem. Chem. Phys. 2005, 7 (18), 3297-3305.

(9) Peterson, K. A.; Figgen, D.; Goll, E.; Stoll, H.; Dolg, M. J. Chem. Phys. 2003, 119 (21), 11113-11123.

(10) Riplinger, C.; Neese, F. J. Chem. Phys. 2013, 138 (3), 34106.

(11) Riplinger, C.; Sandhoefer, B.; Hansen, A.; Neese, F. J. Chem. Phys. 2013, 139 (13), 134101.

(12) Marenich, A. V.; Cramer, C. J.; Truhlar, D. G. J. Phys. Chem. B 2009, 113 (18), 6378-6396. 
(13) Frisch; Trucks, G.; Schlegel, H.; Scuseria, G.; Robb, M.; Cheeseman, J.; Scalmani, G.; Barone, V.; Mennucci, B.; Petersson, G.; Nakatsuji, H.; Caricato, M.; Li, X.; Hratchian, H.; Izmaylov, A.; Bloino, J.; Zheng, G.; Sonnenberg, J.; Hada, M.; Ehara, M.; Toyota, K.; Fukuda, R.; Hasegawa, J.; Ishida, M.; Nakajima, T.; Honda, Y.; Kitao, O.; Nakai, H.; Vreven, T.; Montgomery, J.; Peralta, J.; Ogliaro, F.; Bearpark, M.; Heyd, J.; Brothers, E.; Kudin, K.; Staroverov, V.; Kobayashi, R.; Normand, J.; Raghavachari, K.; Rendell, A.; Burant, J.; lyengar, S.; Tomasi, J.; Cossi, M.; Rega, N.; Millam, J.; Klene, M.; Knox, J.; Cross, J.; Bakken, V.; Adamo, C.; Jaramillo, J.; Gomperts, R.; Stratmann, R.; Yazyev, O.; Austin, A.; Cammi, R.; Pomelli, C.; Ochterski, J.; Martin, R.; Morokuma, K.; Zakrzewski, V.; Voth, G.; Salvador, P.; Dannenberg, J.; Dapprich, S.; Daniels, A.; Farkas; Foresman, J.; Ortiz, J.; Cioslowski, J.; Fox, D. Gaussian 09, Revision D.01, Gaussian, Inc., Wallingford CT. 2013.

Neese, F. Wiley Interdiscip. Rev. Comput. Mol. Sci. 2012, 2 (1), 73-78.

\section{The mechanism of the transformation from $C(I)$ to $D$}

In order to check whether the transformation from $\mathbf{C}(\mathbf{I})$ to $\mathbf{D}$ is concerted or stepwise (through addition/elimination mechanism), we have performed a relaxed potential energy surface scan starting from the intermediate $\mathbf{C}(\mathbf{I})$ and leading to the product $\mathbf{D}$. The results are presented in Figure $\mathbf{S} 2$ below.

The distance $\mathrm{C}_{1}-\mathrm{O}_{1}$ was gradually decreased with the step of $0.025 \AA$, while all other structural parameters were fully optimized at each step. One can see that going via conformational change (C(I)_a, C(I)_b and $\left.\mathbf{C}(\mathbf{I}) \_\mathbf{C}\right)$ and overcoming a barrier with the transition state $\mathbf{T S}_{\mathbf{C}(1)-\mathbf{D}}$ the product $\mathbf{D}$ is obtained without any additional intermediate (this intermediate would be necessary for the addition/elimination mechanism). Thus, we can conclude that the $\mathbf{C}(\mathbf{I}) \rightarrow \mathbf{D}$ step is concerted and has the single transition state $\mathbf{T S}_{\mathbf{C ( I ) - D}}$.

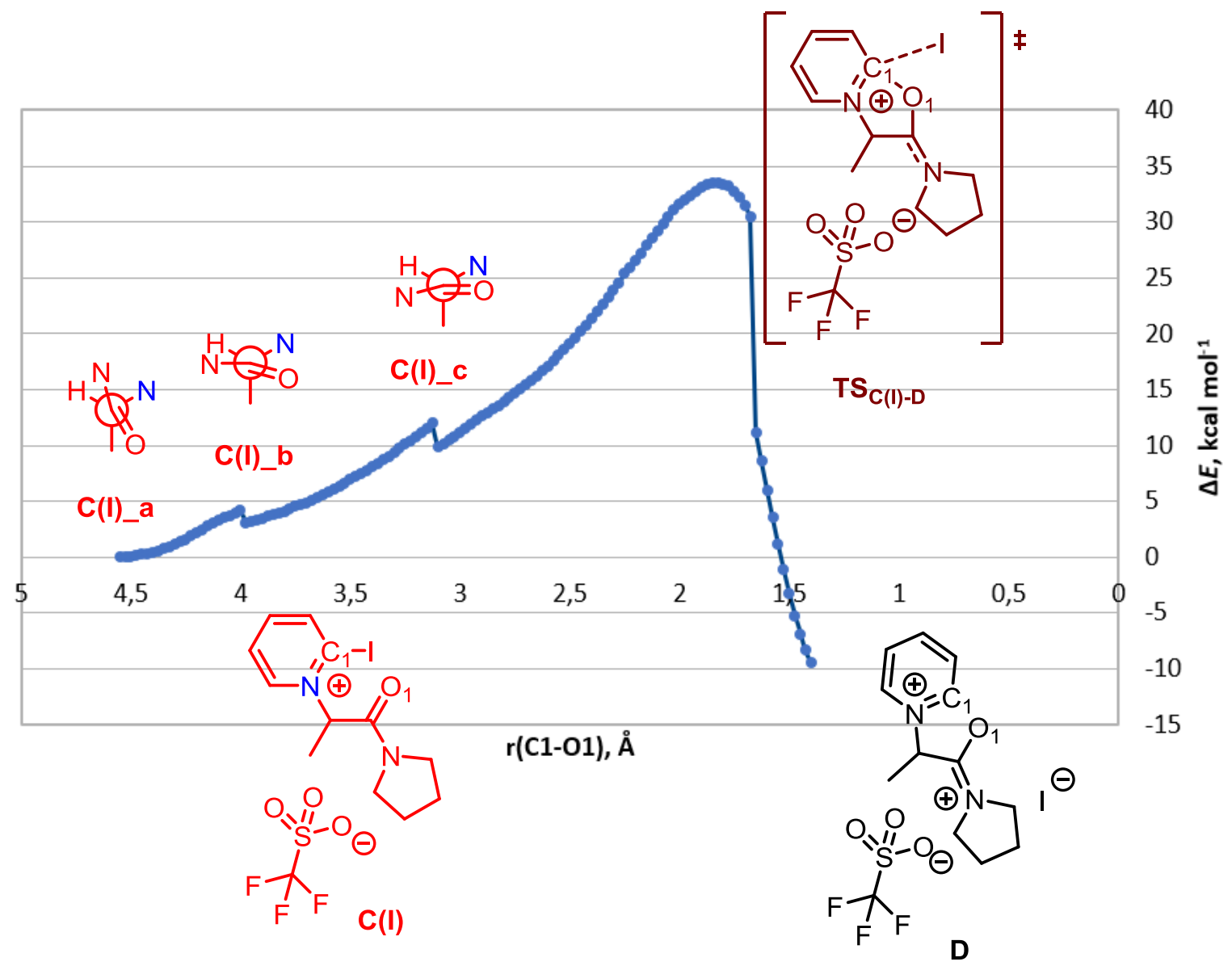

Figure S2 The relaxed potential energy surface scan for the reaction C(I) $\rightarrow$ D (B3LYP-D3-SMD/def2-SVP, ultrafine integration grid 


\begin{tabular}{|c|c|c|c|c|c|c|c|}
\hline \multirow{3}{*}{\multicolumn{4}{|c|}{$\begin{array}{l}\text { Coordinates of the most stable } \\
\left(\Delta G_{298, D C M}\right) \text { conformations as computed } \\
\text { at the }\end{array}$}} & $C$ & -0.37255 & 3.55084 & 1.20504 \\
\hline & & & & $\mathrm{H}$ & -0.19818 & 4.04266 & 2.16239 \\
\hline & & & & $C$ & -0.29712 & 4.25341 & 0.00239 \\
\hline \multicolumn{4}{|c|}{ ef2-SVP level of theory } & $\mathrm{H}$ & -0.36738 & 4.10443 & -2.16408 \\
\hline & & & & $\mathrm{H}$ & -0.07698 & 5.32324 & 0.00882 \\
\hline & & & & $\mathrm{S}$ & 1.96609 & -0.39675 & -0.03606 \\
\hline & -4.89487 & 0.14582 & 0.04982 & $\mathrm{O}$ & 1.87966 & 1.06720 & 0.18325 \\
\hline & -4.96668 & -0.77164 & -0.55449 & $\mathrm{O}$ & 1.41444 & -1.23697 & 1.05286 \\
\hline & -5.35520 & -0.06874 & 1.03195 & $\mathrm{O}$ & 1.65541 & -0.84638 & -1.41536 \\
\hline & -3.47167 & 0.60635 & 0.17602 & $C$ & 3.80731 & -0.71783 & 0.08956 \\
\hline & -3.29346 & 1.64385 & 0.46649 & $\mathrm{~F}$ & 4.27342 & -0.36764 & 1.29655 \\
\hline & -2.42106 & -0.20945 & -0.02189 & $\mathrm{~F}$ & 4.08130 & -2.01607 & -0.09779 \\
\hline & -2.42234 & -1.56175 & -0.19281 & $\mathrm{~F}$ & 4.48226 & -0.01646 & -0.83195 \\
\hline & -2.96723 & -2.46471 & 0.83883 & $\mathrm{H}$ & -5.51669 & 0.92185 & -0.42701 \\
\hline & -4.01074 & -2.74923 & 0.60979 & C & -0.66428 & 1.30889 & 2.40049 \\
\hline & -2.96452 & -1.97190 & 1.82351 & $\mathrm{H}$ & 0.06561 & 0.49584 & 2.25517 \\
\hline & -1.45707 & -2.25511 & -1.07024 & $\mathrm{H}$ & -0.40945 & 1.89335 & 3.29307 \\
\hline & -1.66366 & -2.03059 & -2.13012 & $\mathrm{H}$ & -1.65532 & 0.84879 & 2.53908 \\
\hline & -0.42567 & -1.94233 & -0.85366 & C & -0.82236 & 1.37487 & -2.44541 \\
\hline & -1.66954 & -3.73192 & -0.72248 & $\mathrm{H}$ & -0.03545 & 0.60332 & -2.38728 \\
\hline & -0.77413 & -4.33639 & -0.92993 & $\mathrm{H}$ & -1.79455 & 0.86382 & -2.51470 \\
\hline & -2.50721 & -4.15168 & -1.30534 & $\mathrm{H}$ & -0.67474 & 1.99464 & -3.33831 \\
\hline & -2.03793 & -3.68044 & 0.76607 & & & & \\
\hline & -1.13171 & -3.49073 & 1.36498 & $\mathrm{TS}_{\mathrm{A}-\mathrm{B}}$ & & & \\
\hline & -2.51332 & -4.60133 & 1.13630 & C & 4.93060 & -1.94609 & 0.71711 \\
\hline t & -1.06411 & 0.25974 & -0.01657 & $\mathrm{H}$ & 6.01564 & -1.75594 & 0.71190 \\
\hline & -0.87340 & 1.61005 & -0.01388 & $\mathrm{H}$ & 4.72056 & -2.54570 & 1.62532 \\
\hline$c$ & -0.73929 & 2.22057 & -1.22227 & C & 4.16772 & -0.66864 & 0.74184 \\
\hline & -0.64702 & 2.18687 & 1.19832 & $\mathrm{H}$ & 4.59762 & 0.19722 & 1.25123 \\
\hline & -0.46761 & 3.58541 & -1.21050 & $\mathrm{C}$ & 2.98282 & -0.43512 & 0.05784 \\
\hline
\end{tabular}




\begin{tabular}{|c|c|c|c|c|c|c|c|}
\hline$N$ & 2.05789 & -1.34372 & -0.30557 & $F$ & -4.29351 & -0.70715 & -1.00444 \\
\hline C & 1.79471 & -2.57960 & 0.44837 & $\mathrm{H}$ & 4.67335 & -2.56959 & -0.15187 \\
\hline $\mathrm{H}$ & 1.84773 & -2.38232 & 1.53034 & C & 1.78802 & 2.30618 & -2.40362 \\
\hline $\mathrm{H}$ & 2.53366 & -3.36391 & 0.20911 & $\mathrm{H}$ & 1.37061 & 2.99076 & -3.15323 \\
\hline C & 1.16521 & -1.20806 & -1.47474 & $\mathrm{H}$ & 2.86122 & 2.51398 & -2.28110 \\
\hline $\mathrm{H}$ & 0.44898 & -0.38978 & -1.32449 & $\mathrm{H}$ & 1.69630 & 1.27405 & -2.77116 \\
\hline $\mathrm{H}$ & 1.75342 & -1.01535 & -2.38631 & $\mathrm{C}$ & 1.38171 & 0.91358 & 2.28248 \\
\hline C & 0.42346 & -2.55034 & -1.51208 & $\mathrm{H}$ & 0.89504 & 1.18414 & 3.22768 \\
\hline $\mathrm{H}$ & 0.98062 & -3.28572 & -2.11672 & $\mathrm{H}$ & 2.47035 & 0.99944 & 2.40353 \\
\hline $\mathrm{H}$ & -0.58324 & -2.44265 & -1.93921 & $\mathrm{H}$ & 1.12736 & -0.13132 & 2.05689 \\
\hline C & 0.39980 & -2.97856 & -0.03825 & & & & \\
\hline $\mathrm{H}$ & -0.36023 & -2.39419 & 0.49992 & B & & & \\
\hline $\mathrm{H}$ & 0.19725 & -4.05049 & 0.10380 & C & -1.19152 & 2.61485 & -1.54730 \\
\hline $\mathrm{O}$ & 2.92520 & 0.81471 & -0.37152 & $\mathrm{H}$ & -0.80514 & 3.58751 & -1.21276 \\
\hline$N$ & 1.42146 & 1.73299 & -0.03912 & $\mathrm{H}$ & -1.58091 & 2.69171 & -2.57352 \\
\hline C & 1.06071 & 2.46600 & -1.10325 & C & -0.16734 & 1.53881 & -1.47246 \\
\hline C & 0.87698 & 1.79622 & 1.18566 & $\mathrm{H}$ & -0.44523 & 0.53516 & -1.80515 \\
\hline C & -0.00324 & 3.35894 & -0.93786 & C & 1.06393 & 1.67549 & -0.76663 \\
\hline C & -0.18784 & 2.68572 & 1.36759 & $\mathrm{~N}$ & 1.77187 & 1.68214 & 0.30041 \\
\hline $\mathrm{H}$ & -0.67485 & 2.72581 & 2.34214 & $\mathrm{C}$ & 1.26790 & 1.35177 & 1.66147 \\
\hline C & -0.63401 & 3.45912 & 0.29980 & $\mathrm{H}$ & 0.54328 & 0.53249 & 1.59799 \\
\hline $\mathrm{H}$ & -0.32948 & 3.95358 & -1.79260 & $\mathrm{H}$ & 0.75899 & 2.24409 & 2.05843 \\
\hline $\mathrm{H}$ & -1.48029 & 4.13582 & 0.43189 & C & 3.22328 & 2.01445 & 0.30371 \\
\hline$S$ & -2.18859 & 0.24964 & 0.32474 & $\mathrm{H}$ & 3.75512 & 1.23084 & -0.25326 \\
\hline $\mathrm{O}$ & -1.31602 & -0.38910 & 1.34129 & $\mathrm{H}$ & 3.38249 & 2.97790 & -0.20056 \\
\hline $\mathrm{O}$ & -1.55053 & 0.51429 & -0.98734 & C & 3.57539 & 2.01768 & 1.79564 \\
\hline $\mathrm{O}$ & -3.05809 & 1.33009 & 0.83509 & $\mathrm{H}$ & 3.44460 & 3.03002 & 2.20994 \\
\hline C & -3.41048 & -1.11318 & -0.08282 & $\mathrm{H}$ & 4.61861 & 1.71439 & 1.96102 \\
\hline$F$ & -2.78321 & -2.19905 & -0.56785 & $C$ & 2.55892 & 1.04846 & 2.41945 \\
\hline $\mathrm{F}$ & -4.09231 & -1.48978 & 1.00687 & $\mathrm{H}$ & 2.43513 & 1.19137 & 3.50190 \\
\hline
\end{tabular}




\begin{tabular}{|c|c|c|c|c|c|c|c|}
\hline $\mathrm{H}$ & 2.85956 & 0.00614 & 2.23195 & $C$ & 1.73620 & 3.45640 & 0.81385 \\
\hline 0 & 1.30157 & 1.92616 & -2.00819 & $\mathrm{H}$ & 1.33943 & 4.40340 & 0.41818 \\
\hline $\mathrm{H}$ & -2.01883 & 2.30362 & -0.89275 & $\mathrm{H}$ & 1.46401 & 3.39667 & 1.88107 \\
\hline C & 1.46996 & -2.05070 & 0.41012 & $\mathrm{C}$ & 1.09904 & 2.30251 & 0.12079 \\
\hline C & 0.78735 & -1.97335 & -0.81580 & $\mathrm{H}$ & 0.00880 & 2.21266 & 0.10720 \\
\hline C & 1.50180 & -1.62261 & -1.96082 & $C$ & 1.80135 & 1.12279 & -0.27337 \\
\hline C & 2.87201 & -1.37334 & -1.85925 & $\mathrm{~N}$ & 2.32826 & -0.02428 & -0.06767 \\
\hline C & 3.48731 & -1.49380 & -0.60369 & C & 2.27920 & -0.74696 & 1.22932 \\
\hline$N$ & 2.78882 & -1.81370 & 0.49772 & $\mathrm{H}$ & 1.39293 & -0.42963 & 1.79198 \\
\hline $\mathrm{H}$ & 0.99211 & -1.53788 & -2.92456 & $\mathrm{H}$ & 3.19506 & -0.50468 & 1.79078 \\
\hline $\mathrm{H}$ & -0.28322 & -2.17068 & -0.86181 & C & 2.77621 & -0.90340 & -1.18311 \\
\hline $\mathrm{H}$ & 3.45953 & -1.09226 & -2.73609 & $\mathrm{H}$ & 1.91243 & -1.02560 & -1.85387 \\
\hline $\mathrm{O}$ & -2.37133 & -0.59667 & -1.48588 & $\mathrm{H}$ & 3.60467 & -0.43203 & -1.72986 \\
\hline$S$ & -2.44463 & -0.64862 & -0.00479 & C & 3.14638 & -2.20792 & -0.46894 \\
\hline $\mathrm{O}$ & -2.52891 & -2.00307 & 0.57978 & $\mathrm{H}$ & 4.20935 & -2.19340 & -0.17864 \\
\hline $\mathrm{O}$ & -1.52558 & 0.29181 & 0.68962 & $\mathrm{H}$ & 2.98112 & -3.07902 & -1.11813 \\
\hline C & -4.12822 & 0.08616 & 0.35975 & C & 2.25205 & -2.20775 & 0.78017 \\
\hline $\mathrm{F}$ & -5.09546 & -0.61662 & -0.23765 & $\mathrm{H}$ & 2.60936 & -2.88327 & 1.56993 \\
\hline$F$ & -4.19982 & 1.35173 & -0.08482 & $\mathrm{H}$ & 1.21817 & -2.48619 & 0.52158 \\
\hline $\mathrm{F}$ & -4.36728 & 0.09758 & 1.67534 & 0 & 1.69124 & 1.91278 & -1.28682 \\
\hline C & 0.74817 & -2.40642 & 1.68301 & $S$ & -1.16731 & -0.57517 & 0.16686 \\
\hline $\mathrm{H}$ & 1.09757 & -1.77251 & 2.51276 & $\mathrm{O}$ & -0.45974 & -0.17525 & -1.07711 \\
\hline $\mathrm{H}$ & -0.33888 & -2.29634 & 1.57288 & 0 & -1.31171 & -2.02908 & 0.37696 \\
\hline $\mathrm{H}$ & 0.96638 & -3.45145 & 1.96546 & $\mathrm{O}$ & -0.78149 & 0.21277 & 1.36770 \\
\hline C & 4.96942 & -1.28348 & -0.42818 & C & -2.91305 & 0.01483 & -0.15334 \\
\hline $\mathrm{H}$ & 5.17608 & -0.68824 & 0.47497 & $\mathrm{~F}$ & -2.92398 & 1.33933 & -0.36521 \\
\hline $\mathrm{H}$ & 5.47681 & -2.25441 & -0.29099 & $\mathrm{~F}$ & -3.43111 & -0.58219 & -1.23210 \\
\hline \multirow[t]{2}{*}{$\mathrm{H}$} & 5.42209 & -0.78653 & -1.29874 & $\mathrm{~F}$ & -3.70695 & -0.24599 & 0.89089 \\
\hline & & & & $\mathrm{H}$ & 2.83139 & 3.44057 & 0.72322 \\
\hline
\end{tabular}

B' 


\section{Lutidine}

$\begin{array}{llll}\mathrm{N} & -0.00001 & -0.94113 & 0.00004 \\ \mathrm{C} & 1.16140 & -0.26855 & 0.00554 \\ \mathrm{C} & -1.16141 & -0.26853 & -0.00550 \\ \mathrm{C} & 1.20296 & 1.13532 & 0.00719 \\ \mathrm{C} & -1.20295 & 1.13533 & -0.00718 \\ \mathrm{H} & -2.16232 & 1.65775 & -0.01245 \\ \mathrm{C} & 0.00001 & 1.84276 & -0.00003 \\ \mathrm{H} & 2.16235 & 1.65771 & 0.01249 \\ \mathrm{H} & 0.00002 & 2.93637 & -0.00001 \\ \mathrm{C} & 2.42026 & -1.09676 & -0.00299 \\ \mathrm{H} & 2.50687 & -1.65103 & -0.95330 \\ \mathrm{H} & 2.39043 & -1.84953 & 0.80146 \\ \mathrm{H} & 3.32223 & -0.47990 & 0.12028 \\ \mathrm{C} & -2.42027 & -1.09675 & 0.00296 \\ \mathrm{H} & -2.39042 & -1.84943 & -0.80159 \\ \mathrm{H} & -2.50690 & -1.65111 & 0.95320 \\ \mathrm{H} & -3.32225 & -0.47989 & -0.12028\end{array}$

$\begin{array}{llll}\mathrm{C} & -3.71854 & -0.01297 & 0.80815 \\ \mathrm{H} & -3.63031 & 0.17530 & 1.88760 \\ \mathrm{H} & -4.40926 & 0.72933 & 0.37785 \\ \mathrm{C} & -4.11406 & -1.45046 & 0.45189 \\ \mathrm{H} & -5.20480 & -1.56714 & 0.38713 \\ \mathrm{H} & -3.74058 & -2.14403 & 1.22209 \\ \mathrm{C} & -3.39186 & -1.70592 & -0.87903 \\ \mathrm{H} & -3.92815 & -1.21820 & -1.70978 \\ \mathrm{H} & -3.28494 & -2.77349 & -1.11699 \\ \mathrm{O} & -1.91479 & 2.28626 & 0.96054 \\ \mathrm{~S} & 1.30196 & -0.76802 & -0.10146 \\ \mathrm{O} & 1.51846 & -2.15668 & -0.53485 \\ \mathrm{O} & 0.81373 & 0.15826 & -1.17208 \\ \mathrm{O} & 0.61360 & -0.57909 & 1.19807 \\ \mathrm{C} & 3.01903 & -0.10396 & 0.22960 \\ \mathrm{~F} & 3.61444 & -0.78283 & 1.21372 \\ \mathrm{~F} & 3.78268 & -0.18702 & -0.86356 \\ \mathrm{~F} & 2.94986 & 1.18668 & 0.59335 \\ \mathrm{H} & -1.38783 & 2.77863 & -1.87926\end{array}$

$\mathrm{TS}_{\mathrm{B}^{\prime}-\mathrm{C}(\mathrm{O})}$

$\begin{array}{llll}\mathrm{C} & -0.46586 & 2.81540 & -1.28414 \\ \mathrm{H} & -0.25914 & 3.84814 & -0.96147 \\ \mathrm{H} & 0.38532 & 2.48516 & -1.89957 \\ \mathrm{C} & -0.50991 & 1.92151 & -0.10459 \\ \mathrm{H} & 0.33781 & 1.85860 & 0.57832 \\ \mathrm{C} & -1.69217 & 1.22059 & 0.30733 \\ \mathrm{~N} & -2.39354 & 0.14519 & 0.15415 \\ \mathrm{C} & -2.03580 & -1.03750 & -0.66360 \\ \mathrm{H} & -1.36399 & -1.67328 & -0.06884 \\ \mathrm{H} & -1.50655 & -0.72856 & -1.57149\end{array}$

\section{C(O)}

$\begin{array}{llll}\mathrm{C} & 0.34927 & 2.36617 & 1.50419 \\ \mathrm{H} & 0.79278 & 3.31601 & 1.17114 \\ \mathrm{H} & -0.61775 & 2.57475 & 1.98521 \\ \mathrm{C} & 0.14733 & 1.47590 & 0.29281 \\ \mathrm{H} & -0.53379 & 1.95172 & -0.42509 \\ \mathrm{C} & 1.45131 & 1.23135 & -0.48158 \\ \mathrm{~N} & 2.25028 & 0.19108 & -0.16383 \\ \mathrm{C} & 3.50051 & 0.00358 & -0.92151 \\ \mathrm{H} & 4.16488 & 0.86949 & -0.75592\end{array}$




\begin{tabular}{|c|c|c|c|c|c|c|c|}
\hline $\mathrm{H}$ & 3.29087 & -0.04457 & -2.00070 & $\mathrm{H}$ & 1.43100 & 2.86151 & 0.06903 \\
\hline C & 2.14222 & -0.76844 & 0.95233 & $\mathrm{H}$ & 0.07680 & 3.88968 & 0.63441 \\
\hline $\mathrm{H}$ & 1.75893 & -0.28760 & 1.86105 & $\mathrm{C}$ & -0.95140 & 1.29758 & -1.10036 \\
\hline $\mathrm{H}$ & 1.45610 & -1.59177 & 0.69396 & $\mathrm{H}$ & -0.78264 & 0.21722 & -1.11599 \\
\hline C & 3.57571 & -1.28431 & 1.10082 & $\mathrm{H}$ & -2.03056 & 1.50747 & -1.14684 \\
\hline $\mathrm{H}$ & 3.61270 & -2.27098 & 1.58535 & $\mathrm{C}$ & -0.17133 & 2.05757 & -2.17443 \\
\hline $\mathrm{H}$ & 4.16662 & -0.58039 & 1.71102 & $\mathrm{H}$ & -0.72065 & 2.09677 & -3.12577 \\
\hline C & 4.08598 & -1.28839 & -0.34521 & $\mathrm{H}$ & 0.79532 & 1.55922 & -2.34051 \\
\hline $\mathrm{H}$ & 3.68052 & -2.16100 & -0.88387 & $\mathrm{C}$ & 0.05598 & 3.43920 & -1.54392 \\
\hline $\mathrm{H}$ & 5.18237 & -1.32034 & -0.42437 & $\mathrm{H}$ & -0.86058 & 4.04867 & -1.60762 \\
\hline $\mathrm{O}$ & 1.74018 & 2.04849 & -1.35277 & $\mathrm{H}$ & 0.87502 & 4.00042 & -2.01538 \\
\hline$S$ & -1.22538 & -0.74560 & -0.24270 & 0 & -0.10468 & 1.76070 & 2.46854 \\
\hline $\mathrm{O}$ & -1.16475 & -2.07936 & 0.32682 & $\mathrm{H}$ & 0.66664 & -1.10804 & 1.68901 \\
\hline $\mathrm{O}$ & -0.48661 & 0.24559 & 0.79014 & $\mathrm{C}$ & -2.71188 & -1.93408 & -0.36910 \\
\hline 0 & -0.84263 & -0.45171 & -1.61610 & C & -3.73408 & -2.37431 & -1.22674 \\
\hline C & -2.98835 & -0.12822 & -0.02293 & C & -4.91964 & -1.64712 & -1.30368 \\
\hline $\mathrm{F}$ & -3.41276 & -0.41505 & 1.19735 & $\mathrm{C}$ & -5.05473 & -0.49651 & -0.52739 \\
\hline $\mathrm{F}$ & -3.01207 & 1.18852 & -0.20726 & C & -3.99540 & -0.10899 & 0.30673 \\
\hline $\mathrm{F}$ & -3.76153 & -0.71915 & -0.92151 & $\mathrm{~N}$ & -2.85522 & -0.82239 & 0.37685 \\
\hline \multirow[t]{2}{*}{$\mathrm{H}$} & 1.02348 & 1.89799 & 2.23663 & $\mathrm{H}$ & -5.72863 & -1.96988 & -1.96445 \\
\hline & & & & $\mathrm{H}$ & -3.58896 & -3.27754 & -1.82298 \\
\hline \multicolumn{2}{|c|}{$\mathbf{T S}_{\mathrm{B}^{\prime}-\mathrm{C}(\mathrm{L})}$} & & & $\mathrm{H}$ & -5.96640 & 0.10333 & -0.56474 \\
\hline C & -0.17297 & -0.97986 & 2.37802 & $\mathrm{O}$ & 2.99131 & 0.91513 & -1.37883 \\
\hline $\mathrm{H}$ & 0.20423 & -0.87758 & 3.40984 & $S$ & 2.45993 & 0.03325 & -0.31779 \\
\hline $\mathrm{H}$ & -0.83348 & -1.85733 & 2.37267 & $\mathrm{O}$ & 2.33607 & 0.65541 & 1.02242 \\
\hline C & -0.99173 & 0.21344 & 2.06763 & $\mathrm{O}$ & 1.30882 & -0.81685 & -0.71705 \\
\hline $\mathrm{H}$ & -1.95743 & 0.34366 & 2.55382 & C & 3.81605 & -1.23029 & -0.06116 \\
\hline C & -0.49629 & 1.35195 & 1.33799 & $\mathrm{~F}$ & 3.45966 & -2.11341 & 0.88327 \\
\hline$N$ & -0.41315 & 1.87248 & 0.15540 & $\mathrm{~F}$ & 4.95329 & -0.64299 & 0.32868 \\
\hline C & 0.36998 & 3.11025 & -0.08305 & $\mathrm{~F}$ & 4.06125 & -1.90607 & -1.18979 \\
\hline
\end{tabular}




\begin{tabular}{|c|c|c|c|c|c|c|c|}
\hline C & -1.42507 & -2.70559 & -0.26053 & $\mathrm{H}$ & 0.79448 & -0.59656 & -2.68431 \\
\hline $\mathrm{H}$ & -0.54812 & -2.04475 & -0.31399 & C & -1.87176 & -2.11438 & 0.82608 \\
\hline $\mathrm{H}$ & -1.37623 & -3.24321 & 0.70132 & $C$ & -2.91572 & -2.95555 & 1.23765 \\
\hline $\mathrm{H}$ & -1.34797 & -3.45544 & -1.06100 & $\mathrm{C}$ & -4.18480 & -2.79404 & 0.68655 \\
\hline C & -4.10373 & 1.13668 & 1.14840 & C & -4.38451 & -1.79069 & -0.25789 \\
\hline $\mathrm{H}$ & -5.03982 & 1.67571 & 0.94587 & $\mathrm{C}$ & -3.30905 & -0.97168 & -0.63356 \\
\hline $\mathrm{H}$ & -4.08477 & 0.89248 & 2.22366 & $\mathrm{~N}$ & -2.08526 & -1.14981 & -0.09535 \\
\hline \multirow[t]{2}{*}{$\mathrm{H}$} & -3.26555 & 1.82242 & 0.94800 & $\mathrm{H}$ & -5.00992 & -3.44243 & 0.99222 \\
\hline & & & & $\mathrm{H}$ & -2.72097 & -3.72742 & 1.98445 \\
\hline \multicolumn{2}{|c|}{$\mathrm{TS}_{\mathrm{C}(0)-\mathrm{C}(\mathrm{L})}$} & & & $\mathrm{H}$ & -5.36502 & -1.63015 & -0.71009 \\
\hline C & 0.09274 & -1.05081 & -1.97069 & 0 & 1.78051 & 0.72199 & -0.85819 \\
\hline $\mathrm{H}$ & 0.48446 & -2.01555 & -1.62965 & $S$ & 2.68624 & 0.47929 & 0.34008 \\
\hline $\mathrm{H}$ & -0.85219 & -1.19795 & -2.51151 & 0 & 3.76032 & 1.46817 & 0.44384 \\
\hline C & -0.11031 & -0.10659 & -0.84237 & $\mathrm{O}$ & 1.95009 & 0.11489 & 1.56607 \\
\hline $\mathrm{H}$ & 0.11704 & -0.37434 & 0.17639 & C & 3.54219 & -1.11344 & -0.14753 \\
\hline C & -0.67478 & 1.26216 & -1.15748 & $F$ & 2.63840 & -2.08991 & -0.31675 \\
\hline $\mathrm{N}$ & -1.03295 & 2.03573 & -0.11507 & $\mathrm{~F}$ & 4.39386 & -1.48861 & 0.80662 \\
\hline C & -1.51540 & 3.40287 & -0.34989 & $\mathrm{~F}$ & 4.21321 & -0.95865 & -1.28800 \\
\hline $\mathrm{H}$ & -2.54506 & 3.37072 & -0.75104 & C & -0.49909 & -2.27434 & 1.42489 \\
\hline $\mathrm{H}$ & -0.88730 & 3.90293 & -1.10152 & $\mathrm{H}$ & 0.25569 & -2.51689 & 0.66181 \\
\hline C & -0.94978 & 1.71385 & 1.31946 & $\mathrm{H}$ & -0.16272 & -1.35396 & 1.92646 \\
\hline $\mathrm{H}$ & -1.39203 & 0.73008 & 1.52659 & $\mathrm{H}$ & -0.49669 & -3.08279 & 2.16808 \\
\hline $\mathrm{H}$ & 0.10321 & 1.68640 & 1.63982 & C & -3.51718 & 0.13986 & -1.62262 \\
\hline C & -1.72301 & 2.86033 & 1.97864 & $\mathrm{H}$ & -2.75878 & 0.13051 & -2.41601 \\
\hline $\mathrm{H}$ & -1.39601 & 3.03858 & 3.01330 & $\mathrm{H}$ & -4.51302 & 0.07189 & -2.08096 \\
\hline $\mathrm{H}$ & -2.80015 & 2.62342 & 1.99777 & $\mathrm{H}$ & -3.43843 & 1.11634 & -1.11881 \\
\hline C & -1.46171 & 4.04472 & 1.03920 & & & & \\
\hline $\mathrm{H}$ & -0.45505 & 4.45594 & 1.22170 & $C(L)$ & & & \\
\hline $\mathrm{H}$ & -2.18882 & 4.86203 & 1.15148 & C & -0.80713 & -0.01624 & 1.86063 \\
\hline 0 & -0.77110 & 1.61995 & -2.32973 & $\mathrm{H}$ & -1.65996 & -0.00934 & 2.54877 \\
\hline
\end{tabular}




\begin{tabular}{|c|c|c|c|c|c|c|c|}
\hline $\mathrm{H}$ & -0.34041 & -1.00784 & 1.90432 & $\mathrm{O}$ & 2.58777 & -1.13292 & 1.95670 \\
\hline C & -1.18336 & 0.27228 & 0.39826 & 0 & 1.50466 & -1.48288 & -0.27293 \\
\hline $\mathrm{H}$ & -0.42125 & -0.24141 & -0.18648 & C & 3.98352 & -0.62177 & -0.23670 \\
\hline C & -2.56445 & -0.26330 & -0.02798 & $\mathrm{~F}$ & 3.88406 & -0.13809 & -1.48358 \\
\hline $\mathrm{N}$ & -2.69806 & -1.60335 & -0.07941 & $\mathrm{~F}$ & 4.46589 & -1.86773 & -0.31279 \\
\hline C & -3.98532 & -2.18944 & -0.49229 & $\mathrm{~F}$ & 4.86978 & 0.12840 & 0.42896 \\
\hline $\mathrm{H}$ & -4.21706 & -1.87308 & -1.52395 & C & -2.41416 & 2.46166 & 1.96769 \\
\hline $\mathrm{H}$ & -4.80113 & -1.82452 & 0.15029 & $\mathrm{H}$ & -1.76481 & 2.26934 & 2.83434 \\
\hline C & -1.67138 & -2.63884 & 0.13738 & $\mathrm{H}$ & -3.00706 & 3.35793 & 2.19230 \\
\hline H & -0.68200 & -2.32008 & -0.21758 & $\mathrm{H}$ & -3.10172 & 1.61907 & 1.83883 \\
\hline $\mathrm{H}$ & -1.58467 & -2.86699 & 1.21434 & C & -0.00724 & 0.83583 & -2.11089 \\
\hline C & -2.23895 & -3.84036 & -0.62095 & $\mathrm{H}$ & 0.36587 & 1.25299 & -3.05445 \\
\hline $\mathrm{H}$ & -1.82441 & -4.79342 & -0.26137 & $\mathrm{H}$ & 0.79547 & 0.24292 & -1.65156 \\
\hline $\mathrm{H}$ & -2.00596 & -3.74889 & -1.69541 & $\mathrm{H}$ & -0.84151 & 0.15510 & -2.34202 \\
\hline C & -3.74967 & -3.70111 & -0.38981 & & & & \\
\hline H & -4.00780 & -4.05573 & 0.62187 & \multicolumn{2}{|c|}{ 2-lodopyridine } & & \\
\hline $\mathrm{H}$ & -4.35863 & -4.26614 & -1.11048 & C & 0.60298 & -0.00896 & 0.00061 \\
\hline $\mathrm{O}$ & -3.48675 & 0.50399 & -0.30369 & C & 1.27798 & 1.21941 & 0.00016 \\
\hline $\mathrm{H}$ & -0.04263 & 0.69503 & 2.19584 & C & 2.67331 & 1.17624 & -0.00003 \\
\hline C & -1.61029 & 2.72009 & 0.72820 & C & 3.31947 & -0.06467 & -0.00021 \\
\hline C & -1.40834 & 4.04002 & 0.32439 & C & 2.53562 & -1.21955 & -0.00005 \\
\hline C & -0.70628 & 4.32863 & -0.83934 & $\mathrm{~N}$ & 1.19429 & -1.18546 & 0.00020 \\
\hline C & -0.24735 & 3.27500 & -1.62103 & $\mathrm{H}$ & 3.24792 & 2.10625 & -0.00028 \\
\hline C & -0.44661 & 1.95633 & -1.21905 & $\mathrm{H}$ & 0.73584 & 2.16583 & 0.00001 \\
\hline$N$ & -1.07500 & 1.70477 & -0.02161 & $\mathrm{H}$ & 4.40913 & -0.13707 & -0.00053 \\
\hline $\mathrm{H}$ & -0.54213 & 5.36354 & -1.14766 & $\mathrm{H}$ & 3.00023 & -2.21152 & -0.00034 \\
\hline $\mathrm{H}$ & -1.82617 & 4.83771 & 0.93920 & 1 & -1.55112 & -0.00453 & -0.00006 \\
\hline $\mathrm{H}$ & 0.26810 & 3.45763 & -2.56414 & & & & \\
\hline $\mathrm{O}$ & 1.93664 & 0.83581 & 0.54639 & \multicolumn{2}{|c|}{$\mathrm{TS}_{\mathrm{B}^{\prime}(I)-C(I)}$} & & \\
\hline$S$ & 2.31483 & -0.59762 & 0.61037 & C & -0.82065 & 0.73896 & 2.22146 \\
\hline
\end{tabular}




\begin{tabular}{|c|c|c|c|c|c|c|c|}
\hline $\mathrm{H}$ & -0.55336 & 1.38208 & 3.06956 & $S$ & -3.11163 & 0.02132 & -0.50742 \\
\hline $\mathrm{H}$ & -1.10251 & 1.34795 & 1.34801 & 0 & -2.16904 & 1.15771 & -0.69355 \\
\hline C & 0.30346 & -0.14148 & 1.83819 & 0 & -3.75155 & -0.47493 & -1.74149 \\
\hline $\mathrm{H}$ & 1.31836 & 0.04753 & 2.19191 & $\mathrm{C}$ & -4.51776 & 0.82402 & 0.43133 \\
\hline C & 0.13341 & -1.35983 & 1.09815 & $F$ & -5.47272 & -0.06665 & 0.72002 \\
\hline$N$ & 0.09897 & -1.86601 & -0.08980 & $F$ & -4.07588 & 1.34713 & 1.58592 \\
\hline C & -0.29061 & -3.27919 & -0.30712 & $\mathrm{~F}$ & -5.06797 & 1.81019 & -0.28569 \\
\hline $\mathrm{H}$ & -1.32268 & -3.39708 & 0.05785 & 1 & 3.77849 & -0.45945 & 0.39126 \\
\hline $\mathrm{H}$ & 0.37221 & -3.94994 & 0.25822 & $\mathrm{H}$ & -0.25488 & 2.54973 & -0.17254 \\
\hline C & 0.18041 & -1.10512 & -1.35690 & & & & \\
\hline $\mathrm{H}$ & -0.29530 & -0.12621 & -1.23956 & \multicolumn{2}{|c|}{$\mathrm{TS}_{\mathrm{c}(0)-\mathrm{C}(1)}$} & & \\
\hline $\mathrm{H}$ & 1.24251 & -0.96762 & -1.61235 & $C$ & -0.24973 & -0.30221 & 2.41429 \\
\hline C & -0.53460 & -2.02785 & -2.34627 & $\mathrm{H}$ & -0.23513 & 0.44396 & 3.21830 \\
\hline $\mathrm{H}$ & -0.19938 & -1.85409 & -3.37861 & $\mathrm{H}$ & -1.20642 & -0.84195 & 2.42242 \\
\hline $\mathrm{H}$ & -1.61902 & -1.84381 & -2.29985 & C & -0.08325 & 0.34080 & 1.08520 \\
\hline C & -0.20020 & -3.43344 & -1.82759 & $\mathrm{H}$ & -0.08262 & -0.28964 & 0.20101 \\
\hline $\mathrm{H}$ & 0.82492 & -3.71538 & -2.11925 & $\mathrm{C}$ & 0.10444 & 1.83986 & 0.97123 \\
\hline $\mathrm{H}$ & -0.88663 & -4.20839 & -2.19677 & $\mathrm{~N}$ & 0.25502 & 2.35096 & -0.26330 \\
\hline $\mathrm{O}$ & 0.01578 & -1.88094 & 2.24903 & C & 0.36859 & 3.80605 & -0.44207 \\
\hline $\mathrm{H}$ & -1.71198 & 0.13595 & 2.43979 & $\mathrm{H}$ & 1.17775 & 4.21011 & 0.18546 \\
\hline C & 2.77210 & 1.34137 & -0.21926 & $\mathrm{H}$ & -0.57019 & 4.28690 & -0.11626 \\
\hline C & 3.51027 & 2.32473 & -0.89031 & C & 0.26121 & 1.63571 & -1.54691 \\
\hline C & 2.82246 & 3.46655 & -1.30560 & $\mathrm{H}$ & 1.26122 & 1.20607 & -1.73502 \\
\hline C & 1.45161 & 3.57183 & -1.04889 & $\mathrm{H}$ & -0.46388 & 0.81420 & -1.56527 \\
\hline C & 0.82028 & 2.52984 & -0.37033 & C & -0.07583 & 2.73915 & -2.55107 \\
\hline$N$ & 1.48270 & 1.43797 & 0.04271 & $\mathrm{H}$ & -1.16925 & 2.88148 & -2.57549 \\
\hline $\mathrm{H}$ & 3.35521 & 4.26235 & -1.83249 & $\mathrm{H}$ & 0.26321 & 2.49722 & -3.56875 \\
\hline $\mathrm{H}$ & 4.57657 & 2.19871 & -1.08171 & C & 0.61112 & 3.97084 & -1.94703 \\
\hline $\mathrm{H}$ & 0.87746 & 4.44290 & -1.37068 & $\mathrm{H}$ & 0.21795 & 4.92410 & -2.32900 \\
\hline 0 & -2.64510 & -1.02894 & 0.43187 & $\mathrm{H}$ & 1.69268 & 3.93994 & -2.16089 \\
\hline
\end{tabular}




\begin{tabular}{|c|c|c|c|c|c|c|c|}
\hline 0 & 0.12658 & 2.52853 & 1.99192 & $C$ & -4.74250 & -0.74938 & 0.80903 \\
\hline $\mathrm{H}$ & 0.53873 & -1.05071 & 2.57466 & $\mathrm{H}$ & -5.07083 & 0.15184 & 0.26129 \\
\hline C & 2.83241 & -0.77275 & 0.26663 & $\mathrm{H}$ & -4.98509 & -0.59555 & 1.87071 \\
\hline C & 4.22771 & -0.90217 & 0.29404 & $\mathrm{C}$ & -2.97735 & -2.06396 & -0.25061 \\
\hline C & 4.95282 & -0.03424 & 1.11009 & $\mathrm{H}$ & -2.21551 & -1.78653 & -0.99328 \\
\hline C & 4.27023 & 0.92401 & 1.86491 & $\mathrm{H}$ & -2.59258 & -2.91841 & 0.33260 \\
\hline C & 2.88293 & 0.97980 & 1.76791 & $\mathrm{C}$ & -4.33125 & -2.39219 & -0.88971 \\
\hline$N$ & 2.18660 & 0.14516 & 0.97798 & $\mathrm{H}$ & -4.39354 & -3.44084 & -1.21465 \\
\hline H & 6.04206 & -0.11010 & 1.15481 & $\mathrm{H}$ & -4.49092 & -1.75163 & -1.77327 \\
\hline $\mathrm{H}$ & 4.72506 & -1.66435 & -0.30681 & $\mathrm{C}$ & -5.33535 & -2.02818 & 0.21171 \\
\hline $\mathrm{H}$ & 4.79829 & 1.62012 & 2.51900 & $\mathrm{H}$ & -5.36225 & -2.82273 & 0.97620 \\
\hline O & -2.09583 & 0.74010 & 0.74906 & $\mathrm{H}$ & -6.35952 & -1.88103 & -0.16070 \\
\hline S & -2.83113 & 0.00066 & -0.35749 & 0 & -2.78159 & 0.86214 & 1.92821 \\
\hline O & -3.65238 & 0.88143 & -1.19315 & $\mathrm{H}$ & -0.83995 & -1.07199 & 3.20126 \\
\hline O & -1.99652 & -1.01303 & -1.03009 & $C$ & -0.15173 & 1.66608 & 0.00734 \\
\hline C & -4.05943 & -1.00595 & 0.63402 & $\mathrm{C}$ & 0.49667 & 2.89675 & 0.05010 \\
\hline$F$ & -3.41986 & -1.75962 & 1.53609 & $C$ & 1.11020 & 3.32059 & 1.22589 \\
\hline$F$ & -4.74749 & -1.81057 & -0.17701 & $C$ & 1.05887 & 2.49873 & 2.34973 \\
\hline$F$ & -4.91348 & -0.20916 & 1.27501 & C & 0.40439 & 1.28466 & 2.26116 \\
\hline $\mathrm{H}$ & 2.29874 & 1.71324 & 2.33002 & $\mathrm{~N}$ & -0.18789 & 0.87141 & 1.11505 \\
\hline \multirow[t]{2}{*}{ I } & 1.72324 & -2.12188 & -0.96052 & $\mathrm{H}$ & 1.62548 & 4.28291 & 1.25639 \\
\hline & & & & $\mathrm{H}$ & 0.52653 & 3.51220 & -0.84850 \\
\hline$C(I)$ & & & & $\mathrm{H}$ & 1.52648 & 2.77976 & 3.29372 \\
\hline C & -0.50948 & -1.39819 & 2.20417 & 0 & 2.81895 & 0.85624 & -0.33989 \\
\hline $\mathrm{H}$ & -0.99314 & -2.36324 & 1.99544 & $\mathrm{~S}$ & 2.62175 & -0.51478 & 0.17709 \\
\hline $\mathrm{H}$ & 0.57920 & -1.54335 & 2.18813 & 0 & 2.69409 & -0.65000 & 1.65345 \\
\hline C & -0.93033 & -0.42841 & 1.10339 & 0 & 1.53023 & -1.28378 & -0.46959 \\
\hline $\mathrm{H}$ & -0.67218 & -0.89343 & 0.14825 & $\mathrm{C}$ & 4.15796 & -1.41648 & -0.39701 \\
\hline C & -2.42950 & -0.09439 & 1.24216 & $F$ & 4.14406 & -2.69346 & 0.00816 \\
\hline $\mathrm{N}$ & -3.29259 & -0.93232 & 0.63773 & $\mathrm{~F}$ & 5.26333 & -0.83919 & 0.09266 \\
\hline
\end{tabular}




\begin{tabular}{|c|c|c|c|c|c|c|c|}
\hline $\mathrm{F}$ & 4.24452 & -1.40792 & -1.73411 & C & 0.50407 & -2.25344 & 0.69298 \\
\hline $\mathrm{H}$ & 0.34600 & 0.61052 & 3.10972 & $\mathrm{~N}$ & 1.17626 & -1.05486 & 0.60047 \\
\hline \multirow[t]{2}{*}{ I } & -1.05843 & 1.09451 & -1.80733 & $\mathrm{H}$ & 1.68480 & -3.75039 & -2.09640 \\
\hline & & & & $\mathrm{H}$ & 2.88714 & -1.58914 & -2.26858 \\
\hline \multicolumn{2}{|c|}{$\mathrm{TS}_{\mathrm{C}(1)-\mathrm{D}}$} & & & $\mathrm{H}$ & 0.16386 & -4.19378 & -0.11157 \\
\hline C & 1.27371 & 0.77076 & 2.38932 & $\mathrm{O}$ & -2.83266 & -2.63108 & -0.13989 \\
\hline $\mathrm{H}$ & 2.25717 & 1.18873 & 2.14670 & $S$ & -2.64773 & -1.20134 & 0.16381 \\
\hline $\mathrm{H}$ & 0.64298 & 1.56970 & 2.80509 & $\mathrm{O}$ & -2.40705 & -0.88466 & 1.60172 \\
\hline C & 0.56334 & 0.15357 & 1.18282 & $\mathrm{O}$ & -1.78080 & -0.44446 & -0.77289 \\
\hline $\mathrm{H}$ & -0.45695 & -0.11734 & 1.48213 & $\mathrm{C}$ & -4.34491 & -0.46756 & -0.13415 \\
\hline C & 0.50055 & 1.05270 & -0.05066 & $F$ & -4.35562 & 0.84676 & 0.14349 \\
\hline$N$ & -0.26542 & 2.11198 & -0.11510 & $F$ & -5.26102 & -1.05833 & 0.63999 \\
\hline C & -0.31774 & 2.94991 & -1.33205 & $F$ & -4.71151 & -0.62217 & -1.41086 \\
\hline $\mathrm{H}$ & -0.70566 & 2.33518 & -2.16096 & $\mathrm{H}$ & -0.21349 & -2.31998 & 1.51086 \\
\hline $\mathrm{H}$ & 0.69232 & 3.29127 & -1.59907 & 1 & 4.05036 & 0.15848 & 0.05538 \\
\hline C & -1.33236 & 2.46578 & 0.84848 & & & & \\
\hline $\mathrm{H}$ & -1.83252 & 1.55809 & 1.21037 & D & & & \\
\hline $\mathrm{H}$ & -0.89233 & 3.00489 & 1.70223 & $C$ & 1.27143 & -0.77080 & 2.43420 \\
\hline C & -2.24971 & 3.37073 & 0.02140 & $\mathrm{H}$ & 0.98569 & -1.51448 & 3.19159 \\
\hline $\mathrm{H}$ & -2.81882 & 4.06571 & 0.65488 & $\mathrm{H}$ & 2.28335 & -0.97378 & 2.06066 \\
\hline $\mathrm{H}$ & -2.96493 & 2.74999 & -0.53902 & $\mathrm{C}$ & 0.24818 & -0.80653 & 1.30382 \\
\hline C & -1.28453 & 4.06944 & -0.94467 & $\mathrm{H}$ & -0.76734 & -0.58092 & 1.67366 \\
\hline $\mathrm{H}$ & -0.74071 & 4.87729 & -0.42751 & $\mathrm{C}$ & 0.50249 & 0.08815 & 0.11274 \\
\hline $\mathrm{H}$ & -1.78420 & 4.50177 & -1.82307 & $\mathrm{~N}$ & 0.49352 & 1.36416 & 0.03599 \\
\hline $\mathrm{O}$ & 1.26242 & 0.71942 & -1.01423 & $C$ & 0.63004 & 2.09702 & -1.25399 \\
\hline $\mathrm{H}$ & 1.39133 & -0.00233 & 3.16291 & $\mathrm{H}$ & -0.28518 & 1.88378 & -1.82620 \\
\hline C & 2.06744 & -0.79914 & -0.42057 & $\mathrm{H}$ & 1.51370 & 1.72153 & -1.78695 \\
\hline C & 2.21136 & -1.79278 & -1.43796 & $C$ & 0.30307 & 2.29193 & 1.18053 \\
\hline C & 1.54686 & -2.98855 & -1.32487 & $\mathrm{H}$ & -0.46399 & 1.89085 & 1.85451 \\
\hline C & 0.69977 & -3.25105 & -0.21571 & $\mathrm{H}$ & 1.27124 & 2.37255 & 1.69879 \\
\hline
\end{tabular}




\begin{tabular}{llll}
$\mathrm{C}$ & -0.09326 & 3.59410 & 0.48699 \\
$\mathrm{H}$ & 0.12854 & 4.46684 & 1.11633 \\
$\mathrm{H}$ & -1.17060 & 3.56816 & 0.26556 \\
$\mathrm{C}$ & 0.71835 & 3.55832 & -0.81476 \\
$\mathrm{H}$ & 1.76907 & 3.82733 & -0.62303 \\
$\mathrm{H}$ & 0.32292 & 4.23023 & -1.58889 \\
$\mathrm{O}$ & 0.67327 & -0.63464 & -1.00719 \\
$\mathrm{H}$ & 1.25280 & 0.21926 & 2.90820 \\
$\mathrm{C}$ & 0.46264 & -1.93891 & -0.71951 \\
$\mathrm{C}$ & 0.49193 & -2.98933 & -1.61479 \\
$\mathrm{C}$ & 0.23367 & -4.25561 & -1.08690 \\
$\mathrm{C}$ & -0.03896 & -4.42464 & 0.28121 \\
$\mathrm{C}$ & -0.04537 & -3.32398 & 1.11949 \\
$\mathrm{~N}$ & 0.21256 & -2.10777 & 0.59222 \\
$\mathrm{H}$ & 0.24061 & -5.12277 & -1.75060 \\
$\mathrm{H}$ & -0.24959 & -3.36294 & 2.19040 \\
$\mathrm{H}$ & 0.70327 & -2.80949 & -2.66861 \\
$\mathrm{H}$ & -0.24864 & -5.41094 & 0.69587 \\
$\mathrm{O}$ & -2.50958 & 0.74922 & 1.42908 \\
$\mathrm{~F}$ & -2.74620 & 0.56711 & -0.02796 \\
$\mathrm{O}$ & -2.59926 & 1.78659 & -0.85026 \\
$\mathrm{O}$ & -2.10677 & -0.65410 & -0.58648 \\
$\mathrm{H}$ & -4.57136 & 0.17534 & -0.12633 \\
\hline & -4.94756 & 0.01345 & -1.39823 \\
$\mathrm{H}$ & -4.29255 & 1.16651 & 0.40478 \\
\hline & -0.37577 & -0.13179
\end{tabular}

